\title{
POTENTIAL OFFSITE RADIOLOGICAL DOSES ESTIMATED FOR THE PROPOSED DIVINE STRAKE EXPERIMENT, NEVADA TEST SITE
}

\author{
December 2006
}

Revision 0

Prepared for:

U.S. Department of Energy

National Nuclear Security Administration

Nevada Site Office

Under Contract No. DE-AC52-06NA25946

Prepared by:

National Security Technologies, LLC

Las Vegas, NV 


\section{THIS PAGE INTENTIONALLY LEFT BLANK}




\title{
POTENTIAL OFFSITE RADIOLOGICAL DOSES ESTIMATED FOR THE PROPOSED DIVINE STRAKE EXPERIMENT, NEVADA TEST SITE
}

\author{
December 2006
}

\section{Revision 0}

Reference herein to any specific commercial product, process, or service by trade name, trademark, manufacturer, or otherwise, does not necessarily constitute or imply its endorsement, recommendation, or favoring by the United States Government or any other agency thereof or its contractors or subcontractors. 


\section{THIS PAGE INTENTIONALLY LEFT BLANK}




\section{Executive Summary}

An assessment of the potential radiation dose that residents offsite of the Nevada Test Site (NTS) might receive from the proposed Divine Strake experiment was made to determine compliance with Subpart H of Part 61 of Title 40 of the Code of Federal Regulations, National Emission Standards for Emissions of Radionuclides Other than Radon from Department of Energy Facilities. The Divine Strake experiment, proposed by the Defense Threat Reduction Agency, consists of a detonation of 700 tons of heavy ammonium nitrate fuel oil-emulsion above the U16b Tunnel complex in Area 16 of the NTS. Both natural radionuclides suspended, and historic fallout radionuclides resuspended from the detonation, have potential to be transported outside the NTS boundary by wind. They may, therefore, contribute radiological dose to the public. Subpart H states "Emissions of radionuclides to the ambient air from Department of Energy facilities shall not exceed those amounts that would cause any member of the public to receive in any year an effective dose equivalent of $10 \mathrm{mrem} / \mathrm{yr}$ " (Title 40 of the Code of Federal Regulations [CFR] §61.92) where mrem/yr is millirem per year. Furthermore, application for U.S. Environmental Protection Agency (EPA) approval of construction of a new source or modification of an existing source is required if the effective dose equivalent, caused by all emissions from the new construction or modification, is greater than or equal to $0.1 \mathrm{mrem} / \mathrm{yr}$ (40 CFR $\S 61.96)$.

In accordance with Section 61.93, a dose assessment was conducted with the computer model CAP88-PC, Version 3.0. In addition to this model, a dose assessment was also conducted by the National Atmospheric Release Advisory Center (NARAC) at the Lawrence Livermore National Laboratory. This modeling was conducted to obtain dose estimates from a model designed for acute releases and which addresses terrain effects and uses meteorology from multiple locations.

Potential radiation dose to a hypothetical maximally exposed individual at the closest NTS boundary to the proposed Divine Strake experiment, as estimated by the CAP88-PC model, was 0.005 mrem with wind blowing directly towards that location. Boundary dose, as modeled by NARAC, ranged from about 0.006 to $0.007 \mathrm{mrem}$. Potential doses to actual offsite populated locations were generally two to five times lower still, or about 40 to 100 times lower then the 0.1 mrem level at which EPA approval is required pursuant to Section 61.96. 


\section{THIS PAGE INTENTIONALLY LEFT BLANK}




\section{Table of Contents}

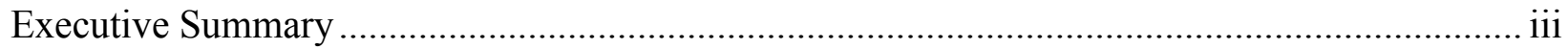

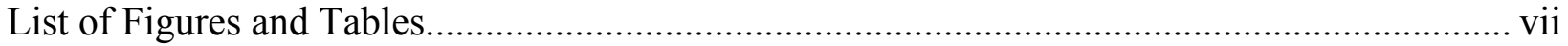

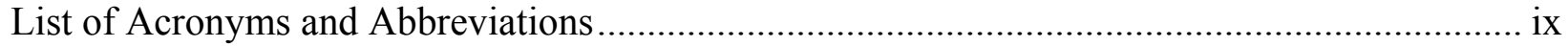

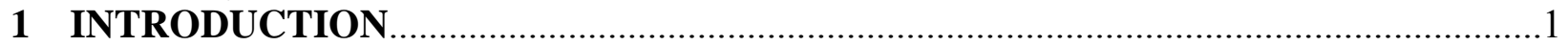

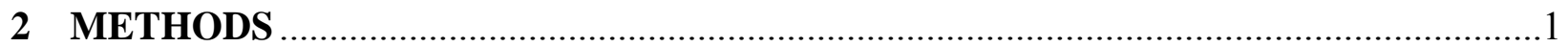

2.1 Clean Air Act Assessment Package - 1988 (CAP88-PC) ............................................ 1

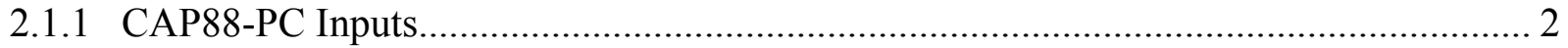

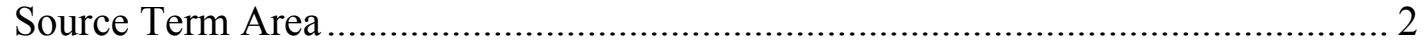

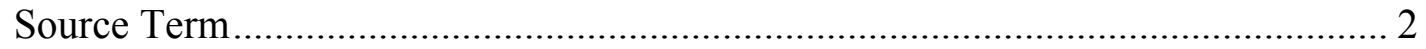

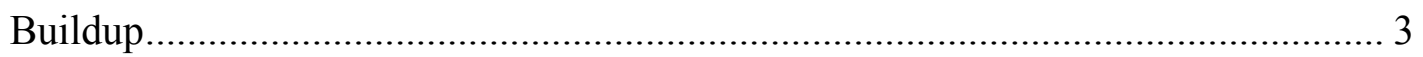

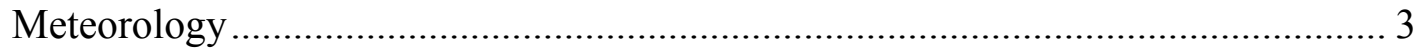

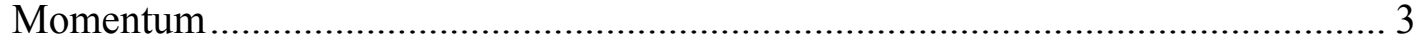

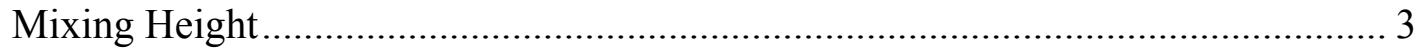

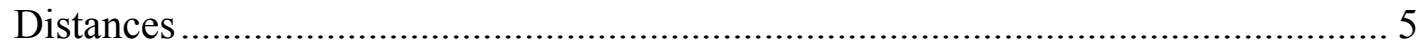

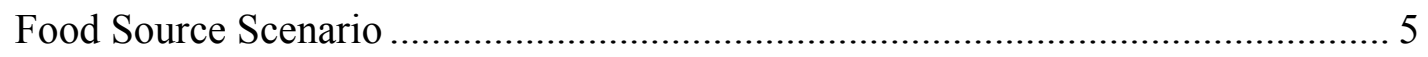

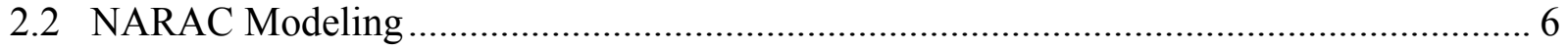

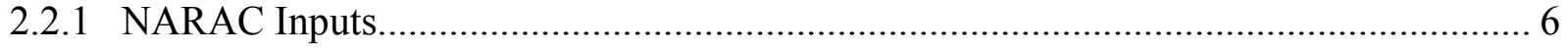

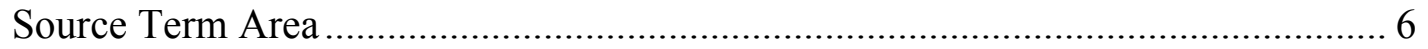

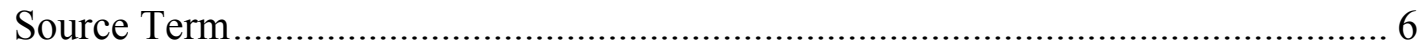

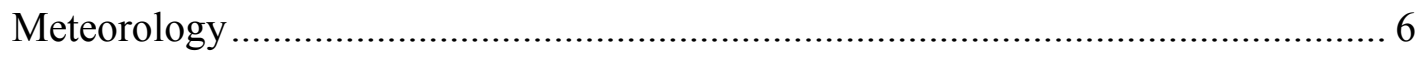

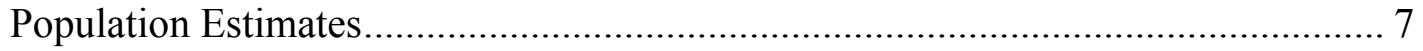

Terrain Source

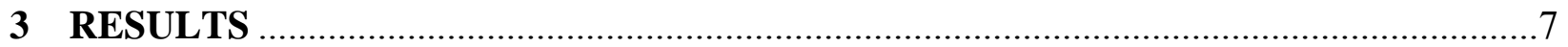

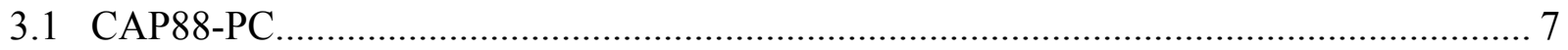

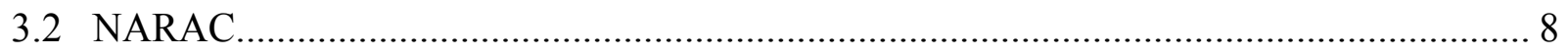

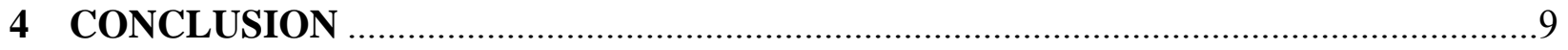

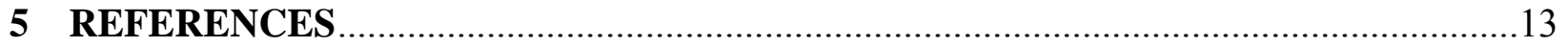

Appendix A: CAP88-PC Version 3.0 Modeling Output for the Proposed Divine Strake

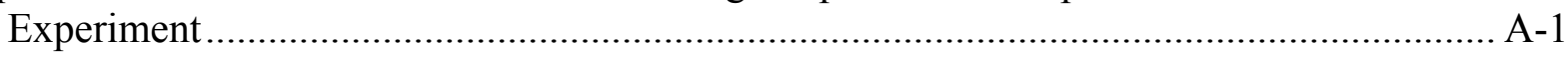

Appendix B: NARAC Modeling Input and Output for the Proposed Divine Strake

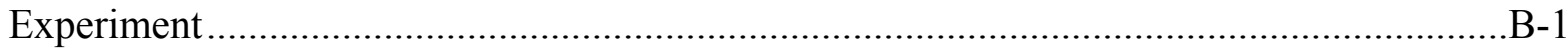

Appendix C: CAP88-PC Version 3.0 Modeling Output for the Proposed Divine Strake

Experiment.....

Appendix D: Output from CAP88-PC Version 3.0 Modtest Sample Case Run

October 2006. 
THIS PAGE INTENTIONALLY LEFT BLANK 


\section{List of Figures and Tables}

Figure 1. NARAC modeled release using meteorology from January 15, 2005 .........................10

Figure 2. NARAC modeled release using meteorology from January 20, 2006 .........................11

Table 1. Source term used for modeling potential dose from the proposed Divine Strake

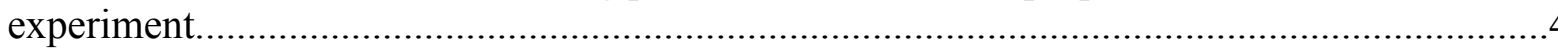

Table 2. Bearing and distance from the proposed Divine Strake experiment .............................. 5

Table 3. Potential dose (mrem) to offsite residents from the proposed Divine Strake

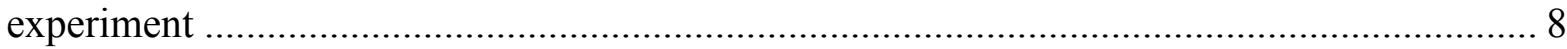




\section{THIS PAGE INTENTIONALLY LEFT BLANK}




\section{List of Acronyms and Abbreviations}

\begin{tabular}{|c|c|}
\hline ANFO & ammonium nitrate fuel oil-emulsion \\
\hline CAP88-PC & Clean Air Act Assessment Package - 1988 \\
\hline CFR & Code of Federal Regulations \\
\hline${ }^{\circ} \mathrm{C}$ & degrees Celcius \\
\hline $\mathrm{Ci}$ & Curie \\
\hline DOE & U.S. Department of Energy \\
\hline DTED & Digital Terrain Elevation Data \\
\hline DTRA & Defense Threat Reduction Agency \\
\hline EPA & U.S. Environmental Protection Agency \\
\hline fps & feet per second \\
\hline $\mathrm{ft}$ & feet \\
\hline $\mathrm{kg}$ & kilogram \\
\hline $\mathrm{km}$ & kilometer \\
\hline LLNL & Lawrence Livermore National Laboratory \\
\hline $\mathrm{m}$ & meter \\
\hline $\mathrm{m}^{2}$ & square meter \\
\hline mrem & milli-Roentgen equivalent man \\
\hline NARAC & National Atmospheric Release Advisory Center \\
\hline NESHAP & National Emission Standards for Hazardous Air Pollutants \\
\hline NTS & Nevada Test Site \\
\hline $\mathrm{pCi} / \mathrm{g}$ & pico-Curies per gram \\
\hline PM10 & particulate matter less than 10 micrometers in diameter \\
\hline PST & Pacific Standard Time \\
\hline S & second \\
\hline UCI & upper confidence interval \\
\hline UTC & Universal Coordinated Time \\
\hline
\end{tabular}




\section{THIS PAGE INTENTIONALLY LEFT BLANK}




\section{INTRODUCTION}

The Defense Threat Reduction Agency (DTRA) plans to conduct an experiment at the U16b Tunnel in Area 16 of the Nevada Test Site (NTS). The experiment, called Divine Strake, will consist of a single detonation of 700 tons (English) of heavy ammonium nitrate fuel oil-emulsion (ANFO) (593 tons trinitrotoluene equivalent) (U.S. Department of Energy [DOE], 2006a). Though no nuclear testing activities of any type were conducted within one mile of the U16b Tunnel (DOE, 2000), the area was subject to fallout from global and NTS nuclear tests. Resuspension of this fallout could travel beyond the NTS boundary where it might contribute to the radiological dose of the public. In addition to man-made radionuclides, the U.S. Environmental Protection Agency (EPA) suggested that the assessment of potential radiological dose also include naturally occurring radionuclides in soils suspended as a result of the explosion (Rosenblum, 2006). This report describes how DOE and DTRA calculated the potential radiological dose to persons residing outside the NTS boundary from radionuclides suspended by the proposed Divine Strake experiment.

Under the Clean Air Act, the National Emission Standards for Hazardous Air Pollutants (NESHAP) were established to control certain pollutants. Subpart H of Part 61 of Title 40 of the Code of Federal Regulations (CFR) states, "Emissions of radionuclides to the ambient air from Department of Energy facilities shall not exceed those amounts that would cause any member of the public to receive in any year an effective dose equivalent of $10 \mathrm{mrem} / \mathrm{yr}$ " (40 CFR §61.92). It is also required that an application for EPA approval of construction of a new source or modification of an existing source be submitted if the effective dose equivalent caused by all emissions from the new construction or modification is equal to or greater than 1 percent of the 10 millirem per year (rem/yr) standard ( $\geq 0.1 \mathrm{mrem} / \mathrm{yr})$ set in 40 CFR $\$ 61.96$. Potential dose from the proposed Divine Strake experiment estimated from models are compared with the limit of $10 \mathrm{mrem} / \mathrm{yr}$ and to the $0.1-\mathrm{mrem} / \mathrm{yr}$ level at which an application for EPA approval is required. Releases of radionuclides to the air from the NTS have been reported annually to the EPA since 1992 in NESHAP reports.

\section{METHODS}

\subsection{Clean Air Act Assessment Package - 1988 (CAP88-PC)}

As specified by Section 61.93, compliance with NESHAP shall be determined and effective dose equivalent values to members of the public calculated using the computer model CAP88-PC, AIRDOS-PC, or other procedures for which EPA has granted prior approval. The use of CAP88-PC has been the most widely used model at facilities within the DOE complex. On February 21, 2006, the EPA announced acceptance of Version 3.0 of the CAP88-PC model for use in demonstrating compliance with the requirements of Subpart H of Part 61 (Federal Register, Vol. 71, pages 8854 - 8856). Version 3.0 of CAP88-PC was one of the models used here to calculate the potential dose to the public from the proposed Divine Strake experiment.

CAPP88-PC uses a modified Gaussian plume equation to estimate dispersal of radionuclide releases. This Gaussian plume model is one of the most commonly used models in government guidebooks, and results have been shown to agree well with experimental data (Shroff, 2006). Since the model was designed for chronic low-level releases, external dose and intake 
assumptions in the model result in a higher, and therefore more conservative, estimate of potential dose to offsite residents when applied to a sudden release like that from the proposed Divine Strake experiment. CAP88-PC does not consider the effects of complex terrain; however, impacts from terrain are expected to be negligible because the size of the proposed Divine Strake experiment release area is small compared with the distances to offsite individuals (Shroff, 2006). Also, potential terrain effects are diminished due to the high release momentum, and therefore, plume height. The National Atmospheric Release Advisory Center (NARAC) modeling described in Section 2.2 of this report addresses issues of the acute release and terrain effects.

Two CAP88-PC runs are described in this report. The only difference between the two is the wind directional speeds used. Wind data are described in the following section. An earlier CAP88-PC run is described in Appendix C. This was based on literature values from historic data for source term whereas the CAP88-PC modeling described in the body of this report is based on site characterization data collected specifically for this purpose (DOE, 2006c). Results from the modeling presented in Appendix $\mathrm{C}$ fall between the modeled results based on the site characterization data.

Prior to modeling potential dose from the proposed Divine Strake experiment, the Modtest sample case described in the CAP88-PC Version 3.0 User Guide, was run on the same computer and software installation used for dose estimations from the proposed Divine Strake experiment. Results of this test were identical to those from the published example (Shroff, 2006), thereby providing an additional measure of quality assurance. The Synopsis and Dose and Risk Equivalent Summary Reports from the Modtest run are provided in Appendix D for comparison with those given in Appendix G of the CAP88-PC Version 3.0 Users Guide (Shroff, 2006).

\subsubsection{CAP88-PC Inputs}

\section{Source Term Area}

The crater created by the proposed Divine Strake experiment is predicted to be 98 feet (ft) (30 meters [m]) in radius (DOE, 2006a). However, particulates are expected to be suspended from the blast over a much larger area. The source term area used in the CAP88-PC modeling was the same as that used for sampling during site characterization (DOE, 2006b), specifically an area of 292,000 square meters $\left(\mathrm{m}^{2}\right)$ (circle with radius of 1,000 $\mathrm{ft}$ or about $300 \mathrm{~m}$ ). The release height above ground level was set to the minimum allowed by CAP88-PC $(0.01 \mathrm{~m})$ since the release is from ground level.

\section{Source Term}

The source term used in modeling is listed in Table 1. Radionuclide concentrations (in picocuries per gram [pCi/g]) in material suspended from the proposed Divine Strake experiment were taken as the maximum upper confidence interval (UCI) for each radionuclide listed in the Site Characterization Report of the Divine Strake Experiment at the Nevada Test Site (DOE, 2006c). These concentrations were multiplied by the total amount of particulate matter less than 10 micrometers in diameter (PM10) predicted to be suspended from the proposed Divine Strake explosion [17.55 tons (English)] (DOE, 2006a). For radionuclides that are part of a decay chain, secular equilibrium was assumed and the maximum UCI within the chain was assumed for the entire decay chain. All specific radionuclides listed in Section 4 of the site characterization 
report (DOE, 2006c) were used as source term regardless of the number of samples that had nondetections.

\section{Buildup}

Because the CAP88-PC model is designed for continuous chronic releases, the default buildup value is 100 years. This means the model allows 100 years for the buildup of short-lived progeny in the environment before potential doses are calculated. While the proposed Divine Strake experiment would be a short-term release, the 100 year buildup value was used as it is the approach specified in the basis model for CAP88-PC (the NUREG 1.109 approach [U.S. Nuclear Regulatory Commission, 1977]) and therefore the approach accepted for demonstrating compliance. The 100 year buildup time is conservative (increases potential dose). In this case, changes in buildup time make a small difference in the calculated dose because progeny within decay chains were assumed to be in equilibrium for the source term for the proposed Divine Strake experiment.

\section{Meteorology}

$\underline{\text { Ambient temperature }}=13.2$ degrees Celsius $\left({ }^{\circ} \mathrm{C}\right)(2005$ average temperature in Area 16) $\underline{\text { Humidity }}=3.4$ grams per cubic meter (average moisture concentration measured by atmospheric moisture samplers on the NTS 2000 - 2002)

$\underline{\text { Precipitation }}=3$ centimeters $(\mathrm{cm})$

Wind: Because it was not known when the proposed Divine Strake experiment would occur, two wind data sets were used. The first represented the worse case scenario with the average actual wind speed and stability class (Class E) observed on the NTS for January $1999-2005$ (6 knots or 3.1 meters per second $[\mathrm{m} / \mathrm{s}]$ ) going in a single direction 100 percent of the time. The second data set used actual stability classes, wind speed, and wind directions in Area 16 of the NTS for all of 2005. The single direction wind file with the relatively low wind speed experienced in January results in a higher (more conservative) dose estimate while the 2005 wind conditions result in a low estimate due to portions of the wind going in all directions over the release period. These two estimates bound the wind conditions expected.

\section{Momentum}

The initial upward momentum of the dust cloud used with CAP88-PC was $4000 \mathrm{~m} / \mathrm{s}$ $(13,000$ feet per second [fps]). This is an approximation for very large quantities of blasting agents which is accepted in the commercial industry (GlobalSecurity.org, 2006).

\section{Mixing Height}

The mixing height used was 3,000 m. This represents the 2,510 m cloud top obtained from Lawrence Livermore National Laboratory's (LLNL's) Hotspot model which was then rounded off to one significant figure. 
Table 1. Source term used for modeling potential dose from the proposed Divine Strake experiment

\begin{tabular}{|c|c|c|c|c|c|}
\hline Analyte & $\begin{array}{c}\text { Concentration for } \\
\text { Source Term } \\
\text { (pCi/g) }\end{array}$ & $\begin{array}{l}\text { Source } \\
\text { Term } \\
\text { (Ci) }^{\mathbf{b}}\end{array}$ & Analyte & $\begin{array}{l}\text { ncentration } \\
\text { Source Term } \\
(\text { pCi/g) }\end{array}$ & $\begin{array}{l}\text { Source Term } \\
\text { (Ci) }\end{array}$ \\
\hline Al-26 & $8.03 \mathrm{E}-02$ & $1.28 \mathrm{E}-06$ & U-235 & $4.23 \mathrm{E}+00$ & $6.73 \mathrm{E}-05$ \\
\hline Am-241 & 7.19E-01 & $1.14 \mathrm{E}-05$ & Th-231 & $4.23 \mathrm{E}+00$ & $6.73 \mathrm{E}-05$ \\
\hline Be-7 & $5.31 \mathrm{E}-01$ & $8.45 \mathrm{E}-06$ & Pa-231 & $4.23 \mathrm{E}+00$ & $6.73 \mathrm{E}-05$ \\
\hline $\mathrm{Cm}-243$ & $4.36 \mathrm{E}-01$ & $6.94 \mathrm{E}-06$ & Ac-227 & $4.23 \mathrm{E}+00$ & $6.73 \mathrm{E}-05$ \\
\hline Co-58 & $4.02 \mathrm{E}-02$ & $6.40 \mathrm{E}-07$ & Th-227 & $4.17 \mathrm{E}+00$ & $6.64 \mathrm{E}-05$ \\
\hline Co-60 & 7.63E-02 & $1.21 \mathrm{E}-06$ & Fr-223 & $5.92 \mathrm{E}-02$ & $9.43 \mathrm{E}-07$ \\
\hline Cs-134 & $3.62 \mathrm{E}-01$ & $5.76 \mathrm{E}-06$ & Ra-223 & $4.23 \mathrm{E}+00$ & $6.73 \mathrm{E}-05$ \\
\hline Cs-137 & $5.50 \mathrm{E}-01$ & $8.76 \mathrm{E}-06$ & Rn-219 & $4.23 \mathrm{E}+00$ & $6.73 \mathrm{E}-05$ \\
\hline Ba-137m & $5.50 \mathrm{E}-01$ & $8.76 \mathrm{E}-06$ & Po-215 & $4.23 \mathrm{E}+00$ & $6.73 \mathrm{E}-05$ \\
\hline Eu-152 & $4.52 \mathrm{E}-01$ & 7.20E-06 & Pb-211 & $4.23 \mathrm{E}+00$ & $6.73 \mathrm{E}-05$ \\
\hline Eu-154 & $3.51 \mathrm{E}-01$ & $5.59 \mathrm{E}-06$ & Bi-211 & $4.23 \mathrm{E}+00$ & $6.73 \mathrm{E}-05$ \\
\hline Eu-155 & $2.18 \mathrm{E}-01$ & $3.47 \mathrm{E}-06$ & Tl-207 & $4.22 \mathrm{E}+00$ & $6.71 \mathrm{E}-05$ \\
\hline H-3 & $8.19 \mathrm{E}+03$ (pCi/L H-3 only) & $1.30 \mathrm{E}-05^{\mathrm{c}}$ & Po-211 & $1.18 \mathrm{E}-02$ & $1.89 \mathrm{E}-07$ \\
\hline K-40 & $1.81 \mathrm{E}+01$ & $2.88 \mathrm{E}-04$ & $\mathrm{U}-238$ & $3.02 \mathrm{E}+00$ & $4.81 \mathrm{E}-05$ \\
\hline Nb-94 & $6.78 \mathrm{E}-02$ & $1.08 \mathrm{E}-06$ & Th-234 & $3.02 \mathrm{E}+00$ & $4.81 \mathrm{E}-05$ \\
\hline Pu-238 & $5.15 \mathrm{E}-02$ & $8.20 \mathrm{E}-07$ & Pa-234m & $3.02 \mathrm{E}+00$ & $4.81 \mathrm{E}-05$ \\
\hline Pu-239 & $2.05 \mathrm{E}+00$ & $3.26 \mathrm{E}-05$ & Pa-234 & $3.93 \mathrm{E}-03$ & $6.25 \mathrm{E}-08$ \\
\hline Sb-125 & $1.83 \mathrm{E}-01$ & 2.91E-06 & U-234 & $3.02 \mathrm{E}+00$ & $4.81 \mathrm{E}-05$ \\
\hline Sr-90 & $1.95 \mathrm{E}-01$ & $3.10 \mathrm{E}-06$ & Th-230 & $3.02 \mathrm{E}+00$ & $4.81 \mathrm{E}-05$ \\
\hline Y-90 & $1.95 \mathrm{E}-01$ & $3.10 \mathrm{E}-06$ & Ra-226 & $3.02 \mathrm{E}+00$ & $4.81 \mathrm{E}-05$ \\
\hline Th-232 & $1.86 \mathrm{E}+00$ & $2.96 \mathrm{E}-05$ & Rn-222 & $3.02 \mathrm{E}+00$ & $4.81 \mathrm{E}-05$ \\
\hline Ra-228 & $1.86 \mathrm{E}+00$ & $2.96 \mathrm{E}-05$ & Po-218 & $3.02 \mathrm{E}+00$ & $4.81 \mathrm{E}-05$ \\
\hline Ac-228 & $1.86 \mathrm{E}+00$ & $2.96 \mathrm{E}-05$ & Pb-214 & $3.02 \mathrm{E}+00$ & 4.81E-05 \\
\hline Th-228 & $1.86 \mathrm{E}+00$ & $2.96 \mathrm{E}-05$ & At-218 & $6.04 \mathrm{E}-04$ & $9.62 \mathrm{E}-09$ \\
\hline Ra-224 & $1.86 \mathrm{E}+00$ & $2.96 \mathrm{E}-05$ & Bi-214 & $3.02 \mathrm{E}+00$ & $4.81 \mathrm{E}-05$ \\
\hline Rn-220 & $1.86 \mathrm{E}+00$ & $2.96 \mathrm{E}-05$ & Рo-214 & $3.02 \mathrm{E}+00$ & $4.81 \mathrm{E}-05$ \\
\hline Po-216 & $1.86 \mathrm{E}+00$ & $2.96 \mathrm{E}-05$ & Pb-210 & $3.02 \mathrm{E}+00$ & $4.81 \mathrm{E}-05$ \\
\hline Pb-212 & $1.86 \mathrm{E}+00$ & $2.96 \mathrm{E}-05$ & Bi-210 & $3.02 \mathrm{E}+00$ & $4.81 \mathrm{E}-05$ \\
\hline Bi-212 & $1.86 \mathrm{E}+00$ & $2.96 \mathrm{E}-05$ & Po-210 & $3.02 \mathrm{E}+00$ & $4.81 \mathrm{E}-05$ \\
\hline Po-212 & $1.19 \mathrm{E}+00$ & $1.90 \mathrm{E}-05$ & & & \\
\hline Tl-208 & 6.70E-01 & $1.07 \mathrm{E}-05$ & & & \\
\hline \multicolumn{6}{|c|}{$\begin{array}{l}\text { a } \mathrm{pCi} / \mathrm{g}=\text { pico Curie per gram: Maximum UCI for each radionuclide listed in the site characterization } \\
\text { report (DOE, 2006c) were used. For radionuclides that are part of a decay chain, secular equilibrium was } \\
\text { assumed and the maximum UCI within the chain was assumed for the entire decay chain. } \\
\mathrm{b} \mathrm{Ci}=\mathrm{Curie} \text { : Concentration for source term multiplied by mass of PM10 material }(15,921,093 \mathrm{~g}) \text {. } \\
{ }^{\mathrm{c}} \text { Tritium associated with soil moisture. Assumed } 10 \text { percent moisture (gravimetric). }\end{array}$} \\
\hline
\end{tabular}




\section{Distances}

Distances entered into CAP88-PC are listed in Table 2 and correspond with distances in kilometers $(\mathrm{km})$ to populated locations outside the NTS boundary. The exception to this is at the closest NTS boundary which is due east of the proposed Divine Strake experiment. There is no public access there due to the U.S. Air Force's Nevada Test and Training Range, but the location was used as a worst case for a hypothetical offsite resident.

\section{Food Source Scenario}

The food source scenario selected for the CAP88-PC modeling was the rural scenario. EPA default values for this scenario were used. The rural scenario results in the highest dose calculation due to the assumption that all food (vegetable, milk, and meat) were produced within the assessment area (i.e., no food was imported).

Table 2. Bearing and distance from the proposed Divine Strake experiment: Latitude $37.0234811^{\circ}$, Longitude $116.1819632^{\circ}$ (NAD 83)

\begin{tabular}{|lccc|}
\hline Location & Distance (km) & Bearing (Degrees) & Sector \\
\hline Eastern NTS Boundary & 22.60 & 90.00 & E \\
Amargosa Valley & 46.71 & 204.15 & SSW \\
American Silica & 48.94 & 154.43 & SSE \\
Springdale & 51.12 & 271.08 & W \\
U.S. Ecology & 53.27 & 240.21 & WSW \\
Beatty & 54.36 & 251.08 & WSW \\
Crystal & 58.78 & 179.31 & S \\
Amargosa Center & 59.11 & 208.56 & SSW \\
Tolicha Peak & 61.71 & 298.81 & WNW \\
Cactus Springs & 63.60 & 139.57 & SE \\
Indian Springs & 67.53 & 136.92 & SE \\
Sarcobatus Flat & 69.59 & 285.80 & WNW \\
Medlin's Ranch & 70.34 & 52.60 & NE \\
Ash Meadows & 73.52 & 188.03 & S \\
Stateline Area & 75.75 & 196.05 & SSW \\
Penoyer Farm & 76.38 & 22.11 & NNE \\
Cold Creek & 78.44 & 149.26 & SSE \\
SNV Prison & 79.13 & 134.46 & SE \\
Rachel & 79.45 & 27.98 & NNE \\
\hline
\end{tabular}




\subsection{NARAC Modeling}

NARAC provides tools and services that map the spread of hazardous material accidentally or intentionally released into the atmosphere. Located at the University of California's LLNL, NARAC is a national support and resource center for planning, preparedness, real-time emergency response, and threat assessments involving nuclear, radiological, chemical, biological, or natural emissions. NARAC predictions provide information on affected areas and populations (NARAC.1lnl.gov, 2006).

The NARAC emergency response system consists of a coupled suite of meteorological and dispersion models. The data assimilation model, Atmospheric Data Assimilation and Parametrization Techniques, constructs variables such as mean winds, pressure, precipitation, temperature, and turbulence, using a variety of interpolation methods and atmospheric parameterizations. Non-divergent wind fields are produced by an adjustment procedure based on the variational principle and a finite-element discretization. The dispersion model, Lagrangian Operational Dispersion Integrator, solves the three-dimensional advection-diffusion equation using a Lagrangian stochastic, Monte Carlo method. LODI includes methods for simulating the processes of mean wind advection, turbulent diffusion, radioactive decay and production, wet deposition, gravitational settling, dry deposition, and buoyant/momentum plume rise. The models are coupled to NARAC databases providing topography, geographical data, and health risk levels. Real-time meteorological observational data, and global and mesoscale forecast model predictions are available. For more information on NARAC, models, and their testing and evaluation see http://narac.llnl.gov/modeling.php (NARAC.llnl.gov, 2006).

Radionuclide transport and potential dose to persons from the proposed Divine Strake experiment were modeled by NARAC for releases beginning on two representative days in January, one during 2005 and one during 2006 (see Meteorology below).

\subsubsection{NARAC Inputs}

\section{Source Term Area}

The source term area used was the same as that described above: a circle with radius rounded to the nearest hundred meters (radius $=300 \mathrm{~m}$ ).

\section{Source Term}

The source term used was the same as described above and listed in Table 1.

\section{Meteorology}

Since it is not known when the proposed Divine Strake experiment will be conducted, meteorology was used from the month of January because, in general, it would provide a higher (more conservative) dose estimate due to average winds blowing in a more northerly direction, lower average wind speeds, and more stable conditions. Specific meteorological data used were from two days in January. The first was January 15, 2005; the second was January 20, 2006. The time of day the models started was 1600 Universal Coordinated Time (UTC), or 0800 
Pacific Standard Time (PST). Data from all available meteorological stations on the NTS plus many off of the NTS were used (see Appendix B for figures showing meteorological stations).

\section{Population Estimates}

Population within estimated plume areas was provided using a database from Los Alamos National Laboratory. The database includes U.S. Census Bureau residential data and business population data (from the State Business Directory). It includes estimates of day-night worker migration, thereby providing a population density database that accounts for time-of-day population variation for the entire United States on a 250-m resolution grid (McPherson and Brown, 2003).

\section{Terrain Source}

The National Imagery and Mapping Agency has developed standard digital datasets, known as Digital Terrain Elevation Data (DTED). DTED are a uniform matrix of terrain elevation values that provide basic quantitative data for systems and applications that require terrain elevation, slope, and/or surface roughness information.

\section{RESULTS}

\subsection{CAP88-PC}

Model results from CAP88-PC are provided in Appendix A and summarized in Table 3. The worst case scenario (wind blowing directly at a hypothetical resident at the closest distance along the east NTS boundary) would result in a dose of 0.005 mrem or 20 times lower than the level at which EPA approval is required. Though the closest NTS boundary was the eastern boundary, potential dose would be very similar at the western boundary (assuming wind blowing towards that direction) given the proposed Divine Strake experiment is nearly centered between the eastern (22.6 km away) and western (23.5 km away) NTS boundaries. The closest offsite population is Amargosa Valley, $46.7 \mathrm{~km}$ to the south-southwest of the proposed Divine Strake experiment. Based on this modeling, wind blowing directly towards Amargosa Valley during the proposed Divine Strake experiment would result in a potential dose of $0.002 \mathrm{mrem}$, about 50 times lower than the level at which EPA approval is required. Release requirements set for the experiment call for surface wind to be blowing from the southwest (240 degrees) through the southeast (120 degrees) at less than 25 miles per hour (DOE, 2006a). In this direction, Medlin's Ranch, Penoyer Farm, and Rachel are the closest populated locations potentially downwind from the experiment. CAP88-PC predicted potential doses of $0.0011,0.0010$, and $0.00096 \mathrm{mrem}$, respectively, at these locations with wind blowing directly at them.

Over 67 percent of the potential dose predicted by CAPP88-PC was from nuclides in the ${ }^{235} \mathrm{U}$ chain. Dose from radionuclides in the ${ }^{232} \mathrm{Th}$ and ${ }^{238} \mathrm{U}$ chains accounted for 11 and 8 percent, of the total dose, respectively. Average concentrations of ${ }^{238} \mathrm{U}$ and ${ }^{235} \mathrm{U}$ reported in the site characterization report for the proposed Divine Strake experiment (DOE, 2006c) are within the range expected for natural uranium (EPA, 1994) and concentration ratios of these averages also suggest natural uranium. The highest contributor to dose from a purely man-made radionuclide was ${ }^{239} \mathrm{Pu}$ which accounted for 9 percent of the total. 
Table 3. Potential dose (mrem) to offsite residents from the proposed Divine Strake experiment based on CAP88-PC Version 3.0 predictions

\begin{tabular}{|c|c|c|c|c|}
\hline Location & $\begin{array}{c}\text { Distance } \\
\text { (km) }\end{array}$ & Sector $^{(a)}$ & $\begin{array}{c}\text { Potential Dose if } \\
\text { Wind Blowing } \\
\text { Directly at Location } \\
\text { (mrem) }\end{array}$ & $\begin{array}{c}\text { Potential Dose if Wind } \\
\text { Blowing at Annual } \\
\text { Average Conditions } \\
\text { (mrem) }\end{array}$ \\
\hline Eastern NTS Boundary & 22.60 & $\mathrm{E}$ & $5.0 \mathrm{E}-03$ & $2.8 \mathrm{E}-04$ \\
\hline Amargosa Valley & 46.71 & SSW & $2.1 \mathrm{E}-03$ & $3.6 \mathrm{E}-05$ \\
\hline American Silica & 48.94 & SSE & $1.9 \mathrm{E}-03$ & $5.4 \mathrm{E}-05$ \\
\hline Springdale & 51.12 & W & $1.8 \mathrm{E}-03$ & 7.4E-05 \\
\hline U.S. Ecology & 53.27 & WSW & $1.7 \mathrm{E}-03$ & $7.8 \mathrm{E}-05$ \\
\hline Beatty & 54.36 & WSW & $1.7 \mathrm{E}-03$ & $7.5 \mathrm{E}-05$ \\
\hline Crystal & 58.78 & $\mathrm{~S}$ & $1.5 \mathrm{E}-03$ & $3.1 \mathrm{E}-05$ \\
\hline Amargosa Center & 59.11 & SSW & $1.4 \mathrm{E}-03$ & $2.0 \mathrm{E}-05$ \\
\hline Tolicha Peak & 61.71 & WNW & $1.3 \mathrm{E}-03$ & $2.1 \mathrm{E}-05$ \\
\hline Cactus Springs & 63.60 & SE & $1.3 \mathrm{E}-03$ & $9.2 \mathrm{E}-05$ \\
\hline Indian Springs & 67.53 & $\mathrm{SE}$ & $1.2 \mathrm{E}-03$ & $8.5 \mathrm{E}-05$ \\
\hline Sarcobatus Flat & 69.59 & WNW & $1.1 \mathrm{E}-03$ & $1.8 \mathrm{E}-05$ \\
\hline Medlin's Ranch & 70.34 & $\mathrm{NE}$ & $1.1 \mathrm{E}-03$ & $3.1 \mathrm{E}-05$ \\
\hline Ash Meadows & 73.52 & $\mathrm{~S}$ & $1.1 \mathrm{E}-03$ & $1.7 \mathrm{E}-05$ \\
\hline Stateline Area & 75.75 & SSW & $1.0 \mathrm{E}-03$ & $1.1 \mathrm{E}-05$ \\
\hline Penoyer Farm & 76.38 & NNE & $1.0 \mathrm{E}-03$ & $4.0 \mathrm{E}-05$ \\
\hline Cold Creek & 78.44 & SSE & $9.7 \mathrm{E}-04$ & $1.8 \mathrm{E}-05$ \\
\hline SNV Prison & 79.13 & $\mathrm{SE}$ & $9.6 \mathrm{E}-04$ & $6.7 \mathrm{E}-05$ \\
\hline Rachel & 79.45 & NNE & $9.6 \mathrm{E}-04$ & $3.8 \mathrm{E}-05$ \\
\hline
\end{tabular}

(a) $\mathrm{E}=$ east, $\mathrm{NE}=$ northeast, $\mathrm{NNE}=$ north-northeast, $\mathrm{S}=$ south, $\mathrm{SE}=$ southeast, $\mathrm{SSE}=$ south-southeast, $\mathrm{SSW}=$ southsouthwest, $\mathrm{W}=$ =west, WNW=west-northwest, WSW=west-southwest

\subsection{NARAC}

Input to and output from the NARAC models are provided in Appendix B. Because of meteorological conditions on days modeled, plume movement generally moved southwest. This was due to mid-level wind conditions. Potential dose from the proposed experiment are displayed in Figure 1 for conditions on January 15, 2005, and in Figure 2 for conditions on January 20, 2006. The modeled dose area extended from the release point out to $60 \mathrm{~km}$ but the edge of the 0.01 mrem dose area extended to less than $25 \mathrm{~km}$ from the release point and was inside the NTS boundaries. The approximate predicted dose value at the NTS boundary for January 15, 2005, was 0.006 mrem and on January 20, 2006, was $0.007 \mathrm{mrem}$. In both cases, potential dose was well below the 0.1-mrem level at which EPA approval is required. Though meteorological conditions on days modeled resulted in the plume moving towards the NTS south and western boundary, potential dose would be very similar at the eastern boundary (assuming similar wind blowing towards that direction) given the proposed Divine Strake experiment is nearly centered between the eastern (22.6 km away) and western (23.5 km away) boundaries. 


\section{CONCLUSION}

Potential radiation dose to a hypothetical maximally exposed individual at the closest NTS boundary to the proposed Divine Strake experiment as estimated by the CAP88-PC model was 0.005 mrem with wind blowing directly towards that location. Boundary dose as modeled by NARAC ranged from 0.006 to $0.007 \mathrm{mrem}$. Potential doses to actual offsite populated locations were generally two to five times lower still, or about 40 to 100 times lower then the 0.1 -mrem level at which EPA approval is required per 40 CFR $\S 61.96$. 


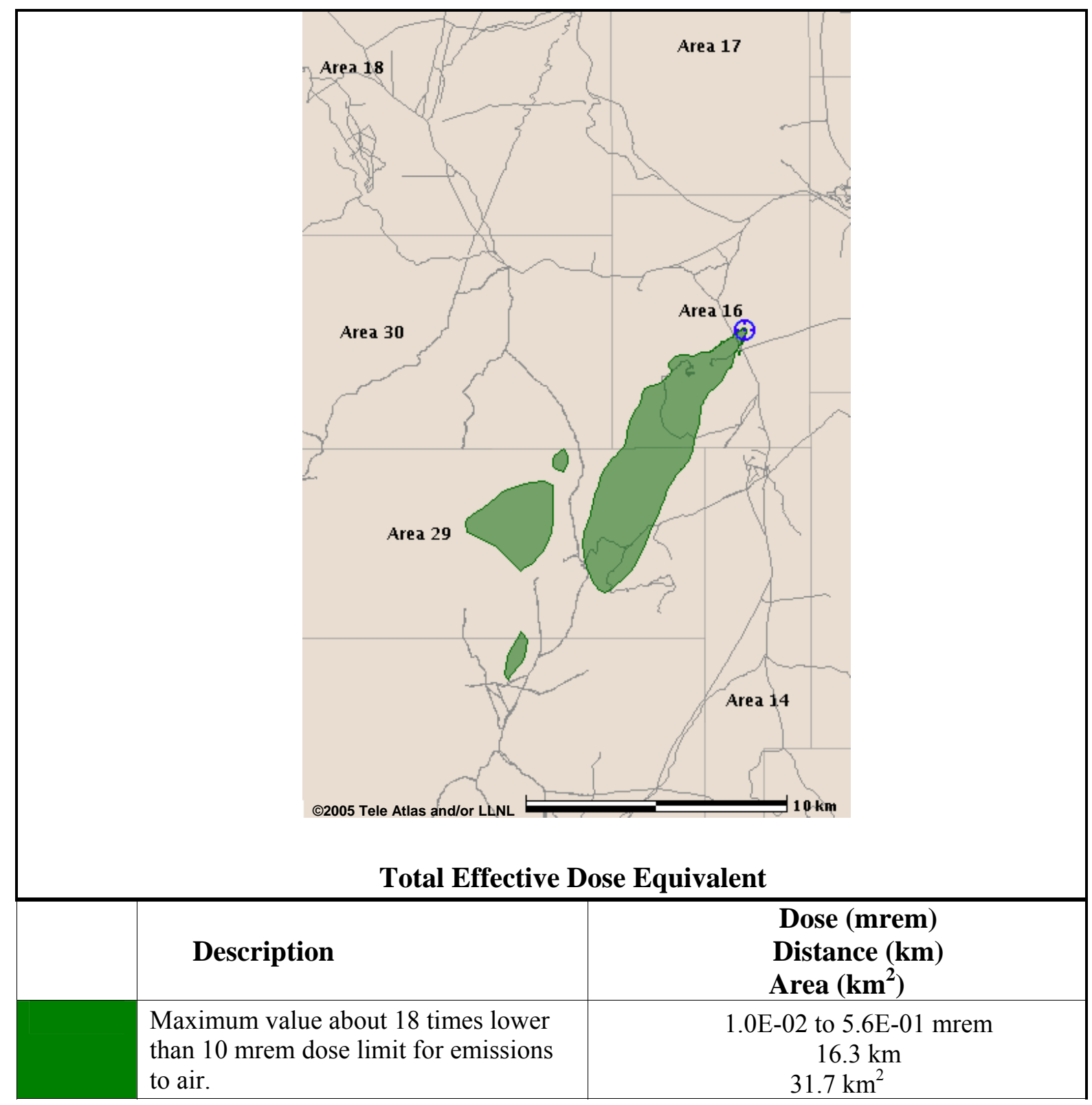

Figure 1. NARAC modeled release using meteorology from January 15, 2005.

Potential dose within the range displayed is completely within the NTS boundaries. Boundary dose based on this modeling was about $0.006 \mathrm{mrem}$. 


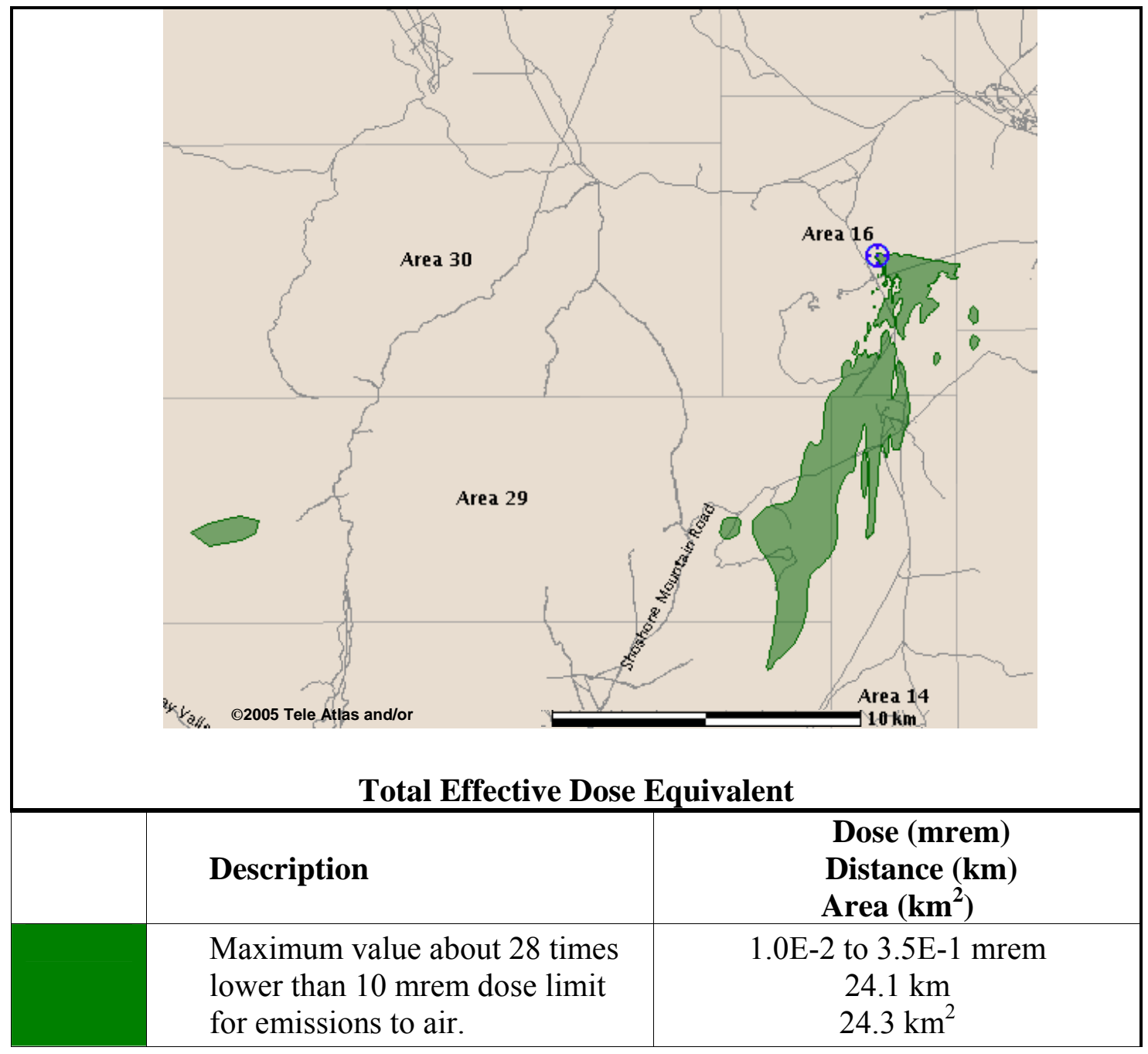

Figure 2. NARAC modeled release using meteorology from January 20, 2006.

Potential dose within the range displayed is completely within the NTS boundaries. Boundary dose based on this modeling was about $0.007 \mathrm{mrem}$. 


\section{THIS PAGE INTENTIONALLY LEFT BLANK}




\section{REFERENCES}

DOE, see U.S. Department of Energy.

EPA, see U.S. Environmental Protection Agency.

Federal Register, 2006. National Emission Standards for Hazardous Air Pollutants (Radionuclides), Availability of Updated Compliance Model. Federal Register, Vol. 71 (February 21, 2006): $8854-8856$.

GlobalSecurity.org, 2006. Explosives - ANFO (Ammonium Nitrate - Fuel Oil) . Available: http://www.globalsecurity.org/military/systems/munitions/explosives-anfo.htm (Accessed 2006, October).

McPherson, T. N. and M. J. Brown, 2003. U.S. Day and Night Population Database UCRL pending Submitted to American Nuclear Society 2006 International Joint Topical Meeting: "Sharing Solutions For Emergencies and Hazardous Environments", Feb. 1215, 2006, Salt Lake City, Utah (Revision 2.0) - Description of Methodology, Report LA-UR-03-8389, Los Alamos National Laboratory, Los Alamos, NM.

NARAC.1lnl.gov, 2006. National Atmospheric Release Advisory Center. Available: http://narac.llnl.gov/ (Accessed 2006, October)

Rosenblum, S., 2006. E-mail message from Shelly Rosenblum, EPA, to Bruce Hurley, NNSA NSO, (Rosenblum.Shelly@epamail.epa.gov; Subject: DIVINE STRAKE - Radionuclide NESHAP), May 17, 2006.

Shroff, B., 2006. CAP88-PC Version 3.0 User Guide. Trinity Engineering Associates, Inc., Cincinnati, OH. March, 2006.

U.S. Department of Energy, 2000. United States Nuclear Tests July 1945 through September 1992. DOE/NV-209 (Rev15), Nevada Operations Office, Las Vegas, NV.

U.S. Department of Energy, 2006a. Large-Scale, Open-Air Explosive Detonation, DIVINE STRAKE, at the Nevada Test Site, Revised Environmental Assessment. DOE/EA-1550, National Nuclear Security Administration Nevada Site Office, Las Vegas, NV.

U.S. Department of Energy, 2006b. Sampling and Analysis Plan for the Divine Strake Experiment Nevada Test Site, Nevada. DOE/NV--1139, National Nuclear Security Administration Nevada Site Office, Las Vegas, NV.

U.S. Department of Energy, 2006c. Site Characterization Report for the Divine Strake Experiment at the Nevada Test Site. DOE/NV-1177, National Nuclear Security Administration Nevada Site Office, Las Vegas, NV.

U.S. Environmental Protection Agency, 1994. Technical Support Document for the Development of Radionuclide Cleanup Levels for Soil. EPA 402-R-96-011 A, Office of Radiation and Indoor Air, Washington, D.C.

U.S. Nuclear Regulatory Commission, 1977. Regulatory Guide 1.109, Calculation of annual Doses to Man from Routine Releases of Reactor Effluents for the purpose of Evaluating Compliance with 10 CFR Part 50, Appendix I. NUREG 1.109. U.S. Nuclear Regulatory Commission, Office of Standards Development, Washington, D.C. 
THIS PAGE INTENTIONALLY LEFT BLANK 


\title{
Appendix A
}

CAP88-PC Version 3.0 Modeling Output for the Proposed Divine Strake Experiment

\author{
October 2006
}

Source term Values Based on Maximum Upper Confidence Intervals from Site Characterization 


\author{
C A P 88 - P C \\ Version 3.0 \\ Clean Air Act Assessment Package - 1988
}

$\begin{array}{lllllllll}S \text { Y N O P S I S R E P O R T } & \end{array}$

Non-Radon Individual Assessment

Oct 26, 2006 08:38 am

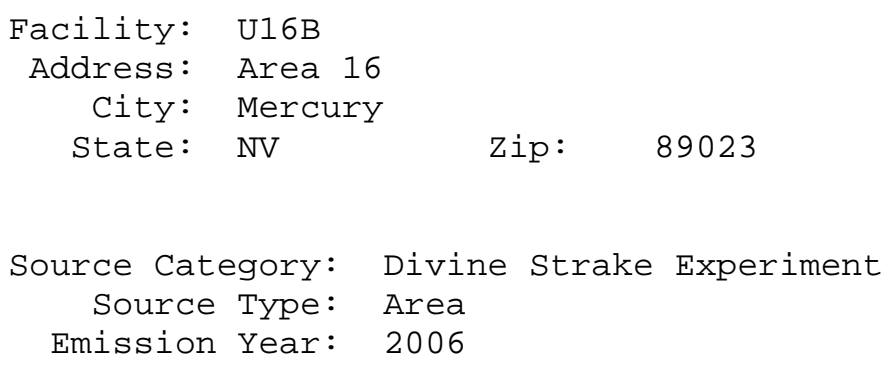

Comments: Dose from the proposed Divine Strake experiment: Single wind direction used. Source used was maximum upper confidence interval reported in D0E/NV-1177 and total PM10

$\begin{gathered}\text { Effective Dose Equivalent } \\ \text { (mrem/year) }\end{gathered}$
$4.98 \mathrm{E}-03$

At This Location: 22600 Meters South Southeast

Dataset Name: DS SNJV Max UCI

Dataset Date: 10/26/2006 7:32:00 AM

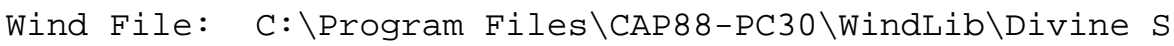


oct 26, $2006 \quad 08: 38$ am

MAXIMALLY EXPOSED INDIVIDUAL

Location of The Individual: 22600 Meters South Southeast

Lifetime Fatal Cancer Risk: 1.61E-09

ORGAN DOSE EQUIVALENT SUMMARY

\begin{tabular}{|c|c|}
\hline Organ & $\begin{array}{c}\text { Dose } \\
\text { Equivalent } \\
(\mathrm{mrem} / \mathrm{y})\end{array}$ \\
\hline Adrenals & $1.33 E-04$ \\
\hline B Surfac & $1.47 \mathrm{E}-\odot 4$ \\
\hline Breasts & $7.37 \mathrm{E}-03$ \\
\hline St Wall & $1.37 \mathrm{E}-\odot 4$ \\
\hline ULI Wall & $1.49 E-04$ \\
\hline Kidneys & $1.29 \mathrm{E}-\odot 4$ \\
\hline Lungs & $1.41 \mathrm{E}-04$ \\
\hline Ovaries & $1.38 \mathrm{E}-04$ \\
\hline $\mathrm{R}$ Marrow & $1.59 E-04$ \\
\hline Spleen & $2.01 E-\odot 4$ \\
\hline Thymus & $2.15 \mathrm{E}-04$ \\
\hline Uterus & $7.38 E-\odot 4$ \\
\hline Bld Wall & $7.46 E-04$ \\
\hline Brain & $1.52 \mathrm{E}-04$ \\
\hline Esophagu & $2.02 \mathrm{E}-04$ \\
\hline SI Wall & $1.33 E-\odot 4$ \\
\hline LLI Wall & $4.37 \mathrm{E}-04$ \\
\hline Liver & $1.78 \mathrm{E}-\odot 3$ \\
\hline Muscle & $1.49 E-04$ \\
\hline Pancreas & $2.19 E-\odot 4$ \\
\hline Skin & $1.38 E-04$ \\
\hline Testes & $1.43 E-\odot 4$ \\
\hline Thyroid & $1.37 \mathrm{E}-04$ \\
\hline EFFEC & $4.98 \mathrm{E}-03$ \\
\hline
\end{tabular}


RADIONUCLIDE EMISSIONS DURING THE YEAR 2006

\begin{tabular}{|c|c|c|c|c|}
\hline Nuclide & Type & Size & $\begin{array}{c}\text { Source } \\
\# 1 \\
\mathrm{Ci} / \mathrm{y}\end{array}$ & $\begin{array}{r}\text { TOTAL } \\
\mathrm{Ci} / \mathrm{y}\end{array}$ \\
\hline Cs -137 & $\mathrm{~F}$ & 1 & $8.8 \mathrm{E}-06$ & $8.8 \mathrm{E}-06$ \\
\hline $\mathrm{Ba}-137 \mathrm{~m}$ & M & 1 & $8.8 \mathrm{E}-06$ & $8.8 E-06$ \\
\hline Am- 241 & M & 1 & 1.1E- 05 & 1. 1E- 05 \\
\hline $\mathrm{Pu}-238$ & M & 1 & $8.2 \mathrm{E}-07$ & $8.2 \mathrm{E}-07$ \\
\hline Pu-239 & M & 1 & $3.3 E-05$ & $3.3 E-05$ \\
\hline $\mathrm{Sr}-90$ & M & 1 & $3.1 \mathrm{E}-06$ & $3.1 \mathrm{E}-06$ \\
\hline Y - $9 \odot$ & M & 1 & $3.1 \mathrm{E}-06$ & $3.1 \mathrm{E}-06$ \\
\hline$K-4 \odot$ & M & 1 & $2.9 \mathrm{E}-\odot 4$ & $2.9 E-\odot 4$ \\
\hline Th- 232 & $S$ & 1 & $3.0 \mathrm{E}-05$ & 3. $0 \mathrm{E}-05$ \\
\hline $\mathrm{Ra}-228$ & M & 1 & $3 . \odot E-\odot 5$ & 3. $0 \mathrm{E}-\odot 5$ \\
\hline$A C-228$ & $M$ & 1 & $3.0 \mathrm{E}-05$ & $3.0 \mathrm{E}-05$ \\
\hline Th-228 & $\mathrm{s}$ & 1 & $3.0 \mathrm{E}-05$ & $3.0 \mathrm{E}-05$ \\
\hline $\mathrm{Ra}-224$ & M & 1 & $3.0 \mathrm{E}-\odot 5$ & 3. $\odot E-\odot 5$ \\
\hline $\mathrm{Rn}-220$ & G & $\odot$ & $3.0 \mathrm{E}-05$ & $3.0 \mathrm{E}-05$ \\
\hline Po-216 & M & 1 & $3.0 \mathrm{E}-05$ & $3.0 \mathrm{E}-05$ \\
\hline $\mathrm{Pb}-212$ & M & 1 & $3.0 E-05$ & 3. $\odot E-\odot 5$ \\
\hline $\mathrm{Bi}-212$ & $M$ & 1 & $3.0 \mathrm{E}-05$ & $3.0 \mathrm{E}-05$ \\
\hline Po- 212 & M & 1 & $1.9 \mathrm{E}-05$ & 1. $9 \mathrm{E}-05$ \\
\hline Tl-208 & M & 1 & $1.1 \mathrm{E}-05$ & 1. $1 \mathrm{E}-05$ \\
\hline$U-238$ & M & 1 & $4.8 \mathrm{E}-05$ & $4.8 E-05$ \\
\hline Th-234 & S & 1 & $4.8 \mathrm{E}-05$ & $4.8 \mathrm{E}-05$ \\
\hline $\mathrm{Pa}-234 \mathrm{~m}$ & M & 1 & $4.8 \mathrm{E}-05$ & $4.8 E-05$ \\
\hline $\mathrm{Pa}-234$ & $M$ & 1 & $6.2 \mathrm{E}-08$ & $6.2 \mathrm{E}-08$ \\
\hline$U-234$ & $M$ & 1 & $4.8 \mathrm{E}-05$ & $4.8 E-05$ \\
\hline Th-230 & S & 1 & $4.8 \mathrm{E}-05$ & $4.8 E-05$ \\
\hline $\mathrm{Ra}-226$ & $M$ & 1 & $4.8 E-05$ & $4.8 E-05$ \\
\hline$R n-222$ & G & $\odot$ & $4.8 \mathrm{E}-05$ & $4.8 E-05$ \\
\hline Po- 218 & M & 1 & $4.8 \mathrm{E}-05$ & $4.8 \mathrm{E}-05$ \\
\hline $\mathrm{Pb}-214$ & M & 1 & $4.8 \mathrm{E}-05$ & $4.8 \mathrm{E}-05$ \\
\hline At -218 & M & 1 & $9.6 \mathrm{E}-09$ & $9.6 \mathrm{E}-\odot 9$ \\
\hline Bi-214 & M & 1 & $4.8 \mathrm{E}-05$ & $4.8 \mathrm{E}-05$ \\
\hline Po-214 & M & 1 & $4.8 \mathrm{E}-05$ & $4.8 \mathrm{E}-05$ \\
\hline $\mathrm{Pb}-210$ & M & 1 & $4.8 \mathrm{E}-05$ & 4. $8 \mathrm{E}-05$ \\
\hline $\mathrm{Bi}-210$ & $M$ & 1 & $4.8 \mathrm{E}-05$ & $4.8 \mathrm{E}-05$ \\
\hline Po-210 & M & 1 & $4.8 \mathrm{E}-05$ & $4.8 \mathrm{E}-05$ \\
\hline U-235 & M & 1 & $6.7 \mathrm{E}-05$ & $6.7 \mathrm{E}-05$ \\
\hline Th-231 & $\mathrm{s}$ & 1 & $6.7 \mathrm{E}-05$ & $6.7 \mathrm{E}-05$ \\
\hline $\mathrm{Pa}-231$ & $M$ & 1 & 6. 7E- -55 & $6.7 \mathrm{E}-05$ \\
\hline$A c-227$ & M & 1 & $6.7 \mathrm{E}-05$ & $6.7 \mathrm{E}-05$ \\
\hline Th -227 & $\mathrm{~s}$ & 1 & $6.6 \mathrm{E}-05$ & $6.6 \mathrm{E}-05$ \\
\hline $\mathrm{Fr}-223$ & M & 1 & $9.4 \mathrm{E}-07$ & $9.4 \mathrm{E}-07$ \\
\hline $\mathrm{Ra}-223$ & M & 1 & $6.7 \mathrm{E}-05$ & $6.7 \mathrm{E}-05$ \\
\hline $\mathrm{Rn}-219$ & G & 0 & $6.7 \mathrm{E}-05$ & $6.7 \mathrm{E}-05$ \\
\hline Po- 215 & $M$ & 1 & 6. $7 \mathrm{E}-05$ & $6.7 \mathrm{E}-05$ \\
\hline $\mathrm{Pb}-211$ & M & 1 & $6.7 \mathrm{E}-05$ & $6.7 \mathrm{E}-05$ \\
\hline
\end{tabular}




$\begin{array}{lllll}\mathrm{Bi}-211 & \mathrm{M} & 1 & 6.7 \mathrm{E}-05 & 6.7 \mathrm{E}-05 \\ \mathrm{Tl}-207 & \mathrm{M} & 1 & 6.7 \mathrm{E}-05 & 6.7 \mathrm{E}-05 \\ \mathrm{PO}-211 & \mathrm{M} & 1 & 1.9 \mathrm{E}-07 & 1.9 \mathrm{E}-07 \\ \mathrm{Al}-26 & \mathrm{M} & 1 & 1.3 \mathrm{E}-06 & 1.3 \mathrm{E}-06 \\ \mathrm{Be}-7 & \mathrm{M} & 1 & 8.4 \mathrm{E}-06 & 8.4 \mathrm{E}-06 \\ \mathrm{Cm}-243 & \mathrm{M} & 1 & 6.9 \mathrm{E}-06 & 6.9 \mathrm{E}-06 \\ \mathrm{C}-58 & \mathrm{M} & 1 & 6.4 \mathrm{E}-07 & 6.4 \mathrm{E}-07 \\ \mathrm{C}-60 & \mathrm{M} & 1 & 1.2 \mathrm{E}-06 & 1.2 \mathrm{E}-06 \\ \mathrm{C}-134 & \mathrm{~F} & 1 & 5.8 \mathrm{E}-06 & 5.8 \mathrm{E}-06 \\ \mathrm{Eu}-152 & \mathrm{M} & 1 & 7.2 \mathrm{E}-06 & 7.2 \mathrm{E}-06 \\ \mathrm{Eu}-154 & \mathrm{M} & 1 & 5.6 \mathrm{E}-06 & 5.6 \mathrm{E}-06 \\ \mathrm{Eu}-155 & \mathrm{M} & 1 & 3.5 \mathrm{E}-06 & 3.5 \mathrm{E}-06 \\ \mathrm{Nb}-94 & \mathrm{M} & 1 & 1.1 \mathrm{E}-06 & 1.1 \mathrm{E}-06 \\ \mathrm{Sb}-125 & \mathrm{M} & 1 & 2.9 \mathrm{E}-06 & 2.9 \mathrm{E}-06 \\ \mathrm{H}-3 & \mathrm{~V} & 0 & 1.3 \mathrm{E}-05 & 1.3 \mathrm{E}-05\end{array}$

\section{SITE INFORMATION}

Temperature: 13 degrees $\mathrm{C}$ Precipitation: $\quad 3 \mathrm{~cm} / \mathrm{y}$

Humidity: $\quad 3 \mathrm{~g} / \mathrm{cu} \mathrm{m}$ Mixing Height: $3000 \mathrm{~m}$ 
SYNOPSIS

Page 3

\section{SOURCE INFORMATION}

Source Number: 1

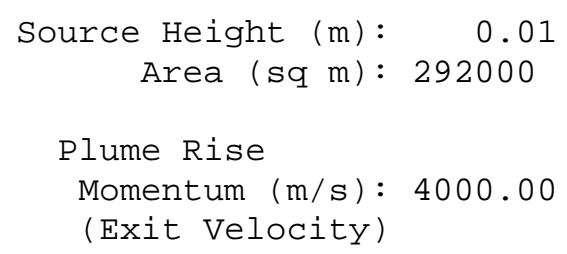

AGRICULTURAL DATA

\begin{tabular}{|c|c|c|c|}
\hline & Vegetable & Milk & Meat \\
\hline Fraction Home Produced: & 0.700 & 0.400 & 0.440 \\
\hline Fraction From Assessment Area: & 0.300 & 0.600 & 0.560 \\
\hline Fraction Imported: & 0.000 & 0.000 & 0.000 \\
\hline
\end{tabular}

Food Arrays were not generated for this run. Default values used.

DISTANCES (M) USED FOR MAXIMUM INDIVIDUAL ASSESSMENT

$\begin{array}{llllllllll}22600 & 46710 & 48940 & 49330 & 51120 & 53270 & 54360 & 58780 & 59110 & 61710 \\ 63600 & 67530 & 69590 & 70340 & 73520 & 75750 & 76380 & 78440 & 79130 & 79450\end{array}$




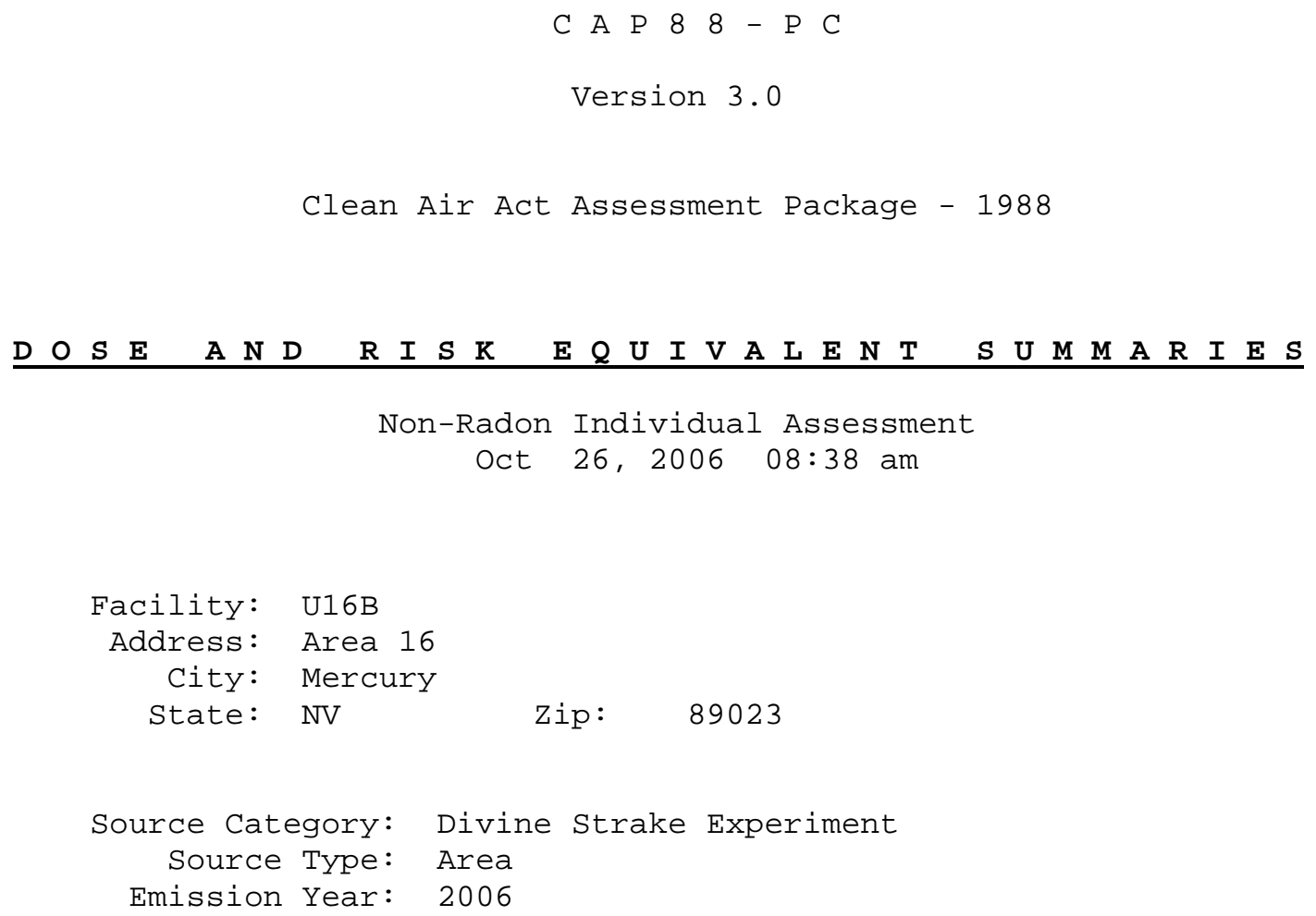

Comments: Dose from the proposed Divine Strake experiment: Single wind direction used. Source used was maximum upper confidence interval reported in DOE/NV-1177 and total PM10.

Stake \6KNTE.WND

Dataset Name: DS SNJV Max UCI

Dataset Date: 10/26/2006 7:32:00 AM

Wind File: . C: $\backslash$ Program Files \CAP88-PC30\WindLib\Divine 
ORGAN DOSE EQUIVALENT SUMMARY

\begin{tabular}{lc} 
Organ & $\begin{array}{c}\text { Selected } \\
\text { Individual } \\
(\mathrm{mrem} / \mathrm{y})\end{array}$ \\
\cline { 2 - 2 } Adrenals & $1.33 \mathrm{E}-\odot 4$ \\
B Surfac & $1.47 \mathrm{E}-\odot 4$ \\
Breasts & $7.37 \mathrm{E}-\odot 3$ \\
St Wall & $1.37 \mathrm{E}-\odot 4$ \\
ULI Wall & $1.49 \mathrm{E}-\odot 4$ \\
Kidneys & $1.29 \mathrm{E}-\odot 4$ \\
Lungs & $1.41 \mathrm{E}-\odot 4$ \\
Ovaries & $1.38 \mathrm{E}-\odot 4$ \\
R Marrow & $1.59 \mathrm{E}-\odot 4$ \\
Spleen & $2.01 \mathrm{E}-\odot 4$ \\
Thymus & $2.15 \mathrm{E}-\odot 4$ \\
Uterus & $7.38 \mathrm{E}-\odot 4$ \\
Bld Wall & $7.46 \mathrm{E}-\odot 4$ \\
Brain & $1.52 \mathrm{E}-\odot 4$ \\
Esophagu & $2.02 \mathrm{E}-\odot 4$ \\
SI Wall & $1.33 \mathrm{E}-\odot 4$ \\
LLI Wall & $4.37 \mathrm{E}-\odot 4$ \\
Liver & $1.78 \mathrm{E}-\odot 3$ \\
Muscle & $1.49 \mathrm{E}-\odot 4$ \\
Pancreas & $2.19 \mathrm{E}-\odot 4$ \\
Skin & $1.38 \mathrm{E}-\odot 4$ \\
Testes & $1.43 \mathrm{E}-\odot 4$ \\
Thyroid & $1.37 \mathrm{E}-\odot 4$ \\
& \\
EFFEC & $4.98 \mathrm{E}-\odot 3$
\end{tabular}

PATHWAY EFFECTIVE DOSE EQUIVALENT SUMMARY

Pathway

INGESTION

INHALATION

AIR IMMERSION

GROUND SURFACE

INTERNAL

EXTERNAL

TOTAL
Selected

Individual

$(\mathrm{mrem} / \mathrm{y})$

$1.48 \mathrm{E}-04$

$4.71 \mathrm{E}-03$

$1.37 \mathrm{E}-08$

1. $20 \mathrm{E}-\mathrm{-} \mathrm{-}$

$4.86 \mathrm{E}-03$

1. $20 \mathrm{E}-\mathrm{\odot} 4$

4. $98 \mathrm{E}-03$ 
Oct 26, $2006 \quad 08: 38$ am

SUMMARY

Page 2

NUCLIDE EFFECTIVE DOSE EQUIVALENT SUMMARY

Nuclide

Cs -137

$\mathrm{Ba}-137 \mathrm{~m}$

Am- 241

$\mathrm{Np}-237$

$\mathrm{Pa}-233$

U-233

Th- 229

$\mathrm{Ra}-225$

Ac- -225

Fr- 221

At -217

$\mathrm{Bi}-213$

Po- 213

$\mathrm{Pb}-209$

Tl-209

$\mathrm{Pu}-238$

U-234

Th- 230

$\mathrm{Ra}-226$

$\mathrm{Rn}-222$

Po- 218

$\mathrm{Pb}-214$

Bi-214

Po- 214

$\mathrm{Pb}-210$

Bi-210

Po- 210

At -218

Sr -90

$\mathrm{Y}-90$

$\mathrm{K}-40$

Th-232

$\mathrm{Ra}-228$

Ac- 228

Th- 228

$\mathrm{Ra}-224$

Rn- 220

Po- 216

$\mathrm{Pb}-212$

Bi-212

Po-212

Tl-208

U-238

Th-234
Selected

Individual

(mrem/y)

1. 23E- 06

2. . $04 \mathrm{E}-06$

1. $23 E-\odot 4$

๑ . ००E+००

$\odot .00 E+\odot \odot$

๑ . ००E+००

$\odot . ~ \odot \odot E+\odot \odot$

๑ . ००E+००

๑. . ०० +००

$\odot . ~ . \odot E+\odot \odot$

๑. . O०E+००

๑ . ००E+००

$\odot . ~ \odot \odot E+\odot \odot$

$\odot . ~ . \odot E+\odot \odot$

$\odot . ~ \odot \odot E+\odot \odot$

9. 79E- 06

4. 42E- 05

1. $77 \mathrm{E}-04$

6. . $01 E-05$

1. $04 \mathrm{E}-12$

1. $78 \mathrm{E}-10$

5. 10E-06

2. $98 \mathrm{E}-05$

1. $63 \mathrm{E}-09$

3. 28E- $\odot 5$

1. 89E- 06

4. 66E- 05

9. $43 \mathrm{E}-16$

8. 57E- 07

1. $46 \mathrm{E}-07$

4. 88E- 05

1. $91 \mathrm{E}-04$

4. 64E- 05

1. 20E- 05

3. $02 \mathrm{E}-04$

2. 28E- $\odot 5$

6. $20 \mathrm{E}-13$

2. $04 \mathrm{E}-10$

3. . $01 E-06$

3. $08 \mathrm{E}-06$

$\odot . \odot \odot E+\odot \odot$

1. 35E- 05

3. 64E- 05

2. 87E- 07 


\begin{tabular}{|c|c|}
\hline $\mathrm{Pa}-234 \mathrm{~m}$ & $2.21 \mathrm{E}-06$ \\
\hline $\mathrm{Pa}-234$ & $1.17 \mathrm{E}-11$ \\
\hline Al-26 & $1.36 \mathrm{E}-06$ \\
\hline $\mathrm{Be}-7$ & $7.67 \mathrm{E}-\odot 8$ \\
\hline $\mathrm{Cm}-243$ & $5.68 \mathrm{E}-\odot 5$ \\
\hline Am - 243 & $\odot . \odot \odot \mathrm{E}+\odot \odot$ \\
\hline$N p-239$ & $\odot . \odot \odot E+\odot \odot$ \\
\hline Pu-239 & $4.23 E-04$ \\
\hline U-235 & $5.89 \mathrm{E}-05$ \\
\hline Th-231 & $4.54 \mathrm{E}-07$ \\
\hline $\mathrm{Pa}-231$ & $1.63 \mathrm{E}-03$ \\
\hline Ac - 227 & $1.27 \mathrm{E}-\odot 3$ \\
\hline Th - 227 & $1.79 \mathrm{E}-\odot 4$ \\
\hline $\mathrm{Ra}-223$ & $1.35 E-04$ \\
\hline$R n-219$ & $2.02 \mathrm{E}-10$ \\
\hline Po-215 & $4.82 \mathrm{E}-\odot 9$ \\
\hline $\mathrm{Pb}-211$ & $2.91 \mathrm{E}-06$ \\
\hline Bi-211 & $1.26 \mathrm{E}-06$ \\
\hline Tl-207 & $1.59 \mathrm{E}-06$ \\
\hline Po-211 & $6.74 \mathrm{E}-14$ \\
\hline$F r-223$ & $3.33 E-\odot 8$ \\
\hline Co-58 & $8.51 \mathrm{E}-\odot 8$ \\
\hline Co-60 & $1.19 \mathrm{E}-06$ \\
\hline Cs -134 & $4.4 \odot E-\odot 6$ \\
\hline Eu-152 & $8.62 \mathrm{E}-08$ \\
\hline$G d-152$ & $\odot . \odot \odot E+\odot \odot$ \\
\hline Eu-154 & $7.95 E-\odot 8$ \\
\hline Eu-155 & $8.48 \mathrm{E}-\odot 8$ \\
\hline $\mathrm{Nb}-94$ & $6.92 \mathrm{E}-07$ \\
\hline$S b-125$ & $4.87 \mathrm{E}-07$ \\
\hline $\mathrm{Te}-125 \mathrm{~m}$ & $3.06 \mathrm{E}-09$ \\
\hline $\mathrm{H}-3$ & $4.29 \mathrm{E}-10$ \\
\hline TOTAL & $4.98 \mathrm{E}-03$ \\
\hline
\end{tabular}


CANCER RISK SUMMARY

\begin{tabular}{lc} 
& $\begin{array}{c}\text { Selected Individual } \\
\text { Total Lifetime } \\
\text { Fatal Cancer Risk }\end{array}$ \\
& \\
\cline { 2 - 2 } Esophagu & $4.16 \mathrm{E}-12$ \\
Stomach & $1.20 \mathrm{E}-11$ \\
Colon & $3.97 \mathrm{E}-11$ \\
Liver & $1.39 \mathrm{E}-10$ \\
LUNG & $1.22 \mathrm{E}-09$ \\
Bone & $8.15 \mathrm{E}-11$ \\
Skin & $1.96 \mathrm{E}-12$ \\
Breast & $1.04 \mathrm{E}-11$ \\
Ovary & $1.69 \mathrm{E}-11$ \\
Bladder & $1.00 \mathrm{E}-11$ \\
Kidneys & $8.67 \mathrm{E}-12$ \\
Thyroid & $9.61 \mathrm{E}-13$ \\
Leukemia & $2.23 \mathrm{E}-11$ \\
Residual & $4.40 \mathrm{E}-11$ \\
Total & $1.61 \mathrm{E}-09$ \\
ToTAL & $3.23 \mathrm{E}-09$ \\
&
\end{tabular}

PATHWAY RISK SUMMARY

Pathway

INGESTION

INHALATION

AIR IMMERSION

GROUND SURFACE

INTERNAL

EXTERNAL

TOTAL
Selected Individual Total Lifetime Fatal Cancer Risk

$5.73 \mathrm{E}-11$

1. $50 \mathrm{E}-09$

7. $41 \mathrm{E}-15$

$5.89 \mathrm{E}-11$

1. $55 \mathrm{E}-09$

$5.89 \mathrm{E}-11$

1. 61E- 09 
Nuclide

Cs -137

$\mathrm{Ba}-137 \mathrm{~m}$

Am- 241

$\mathrm{Np}-237$

$\mathrm{Pa}-233$

U -233

Th- 229

$\mathrm{Ra}-225$

Ac -225

Fr -221

At -217

Bi- 213

Po -213

$\mathrm{Pb}-209$

Tl- 209

$\mathrm{Pu}-238$

$\mathrm{U}-234$

Th -230

$\mathrm{Ra}-226$

$\mathrm{Rn}-222$

Po- 218

$\mathrm{Pb}-214$

Bi-214

Po- 214

$\mathrm{Pb}-210$

Bi -210

Po- 210

At -218

$\mathrm{Sr}-90$

$\mathrm{Y}-90$

$\mathrm{K}-40$

Th- 232

$\mathrm{Ra}-228$

Ac -228

Th -228

$\mathrm{Ra}-224$

$\mathrm{Rn}-220$

Po- 216

$\mathrm{Pb}-212$

Bi- 212

Po -212

$\mathrm{Tl}-208$

$\mathrm{U}-238$

Th-234
Selected Individual Total Lifetime Fatal Cancer Risk

$6.18 \mathrm{E}-13$

$1.10 \mathrm{E}-12$

1. $94 \mathrm{E}-11$

$\odot .00 \mathrm{E}+\odot \odot$

$\odot .0 \odot \mathrm{E}+\odot \odot$

$\odot .0 \odot E+\odot \odot$

$\odot .0 \odot \mathrm{E}+\odot \odot$

$\odot .00 \mathrm{E}+\odot \odot$

$\odot .0 \odot E+\odot \odot$

$\odot .00 \mathrm{E}+\odot \odot$

$\odot .00 \mathrm{E}+\odot \odot$

$\odot .00 \mathrm{E}+\odot \odot$

$\odot .00 \mathrm{E}+\odot \odot$

$\odot .00 E+\odot \odot$

$\odot .00 \mathrm{E}+\odot \odot$

1. 70E-12

3. $62 \mathrm{E}-11$

8. $96 \mathrm{E}-11$

4. $21 \mathrm{E}-11$

5. 66E-19

9. $74 \mathrm{E}-17$

2. $74 \mathrm{E}-12$

1. $58 \mathrm{E}-11$

8. $92 \mathrm{E}-16$

1. $48 \mathrm{E}-11$

1. $10 \mathrm{E}-12$

3. $64 \mathrm{E}-11$

4. $58 \mathrm{E}-22$

5. $06 \mathrm{E}-13$

1. $85 \mathrm{E}-14$

3. 31E-11

8. $38 \mathrm{E}-11$

1. $99 \mathrm{E}-11$

6. $37 \mathrm{E}-12$

2. $58 \mathrm{E}-10$

1. $95 \mathrm{E}-11$

3. 39E-19

1. $12 \mathrm{E}-16$

2. $05 \mathrm{E}-12$

1. $43 \mathrm{E}-12$

$\odot .00 \mathrm{E}+\odot \odot$

7. 36E-12

2. $97 \mathrm{E}-11$

2. $24 \mathrm{E}-13$ 
$\mathrm{Pa}-234 \mathrm{~m}$

$\mathrm{Pa}-234$

Al-26

$\mathrm{Be}-7$

Cm- 243

Am- 243

$\mathrm{Np}-239$

$\mathrm{Pu}-239$

U-235

Th-231

$\mathrm{Pa}-231$

Ac -227

Th-227

Ra-223

Rn-219

Po- 215

$\mathrm{Pb}-211$

Bi-211

Tl-207

Po-211

$\mathrm{Fr}-223$

Co-58

Co- 60

Cs-134

Eu-152

Gd-152

Eu-154

Eu-155

$\mathrm{Nb}-94$

$\mathrm{Sb}-125$

Te-125m

H-3

TOTAL
3. $55 \mathrm{E}-13$

7. $48 \mathrm{E}-18$

7. $41 \mathrm{E}-13$

4. $20 \mathrm{E}-14$

1. 17E-11

$\odot .00 E+0 \odot$

$\odot .00 \mathrm{E}+\odot \odot$

6. 69E-11

4. $71 \mathrm{E}-11$

2. $08 \mathrm{E}-13$

1. $55 \mathrm{E}-10$

3. $33 \mathrm{E}-10$

1. $55 \mathrm{E}-10$

1. $14 \mathrm{E}-10$

1. $09 \mathrm{E}-16$

2. $64 \mathrm{E}-15$

1. $07 \mathrm{E}-12$

6. $91 \mathrm{E}-13$

2. $03 \mathrm{E}-13$

3. $69 \mathrm{E}-20$

1. $38 \mathrm{E}-14$

4. $70 \mathrm{E}-14$

6. $75 \mathrm{E}-13$

2. $36 \mathrm{E}-12$

4. $21 \mathrm{E}-14$

$\odot .00 \mathrm{E}+\odot \odot$

3. $98 \mathrm{E}-14$

4. $50 \mathrm{E}-14$

3. $81 \mathrm{E}-13$

2. $67 \mathrm{E}-13$

1. 39E-15

2. $64 \mathrm{E}-16$

1. 61E- 09 
Oct 26, $2006 \quad 08: 38$ am

SUMMARY

Page 5

INDIVIDUAL EFFECTIVE DOSE EQUIVALENT RATE ( $\mathrm{mrem} / \mathrm{y}$ )

(All Radionuclides and Pathways)

\begin{tabular}{|c|c|c|c|c|c|c|c|}
\hline \multirow[b]{2}{*}{ Direction } & \multicolumn{6}{|c|}{ Distance $(\mathrm{m})$} & \multirow[b]{2}{*}{54360} \\
\hline & 22600 & 46710 & 48940 & 49330 & 51120 & 53270 & \\
\hline $\mathrm{N}$ & $6.3 \mathrm{E}-12$ & $6.3 \mathrm{E}-12$ & $6.3 \mathrm{E}-12$ & $6.3 \mathrm{E}-12$ & $6.3 \mathrm{E}-12$ & $6.3 \mathrm{E}-12$ & $6.3 \mathrm{E}-12$ \\
\hline NNW & $6.3 \mathrm{E}-12$ & $6.3 \mathrm{E}-12$ & $6.3 \mathrm{E}-12$ & $6.3 \mathrm{E}-12$ & $6.3 \mathrm{E}-12$ & $6.3 \mathrm{E}-12$ & $6.3 \mathrm{E}-12$ \\
\hline NW & $6.3 \mathrm{E}-12$ & $6.3 E-12$ & $6.3 \mathrm{E}-12$ & $6.3 \mathrm{E}-12$ & $6.3 \mathrm{E}-12$ & $6.3 \mathrm{E}-12$ & $6.3 E-12$ \\
\hline WNW & $6.3 \mathrm{E}-12$ & $6.3 \mathrm{E}-12$ & $6.3 E-12$ & $6.3 \mathrm{E}-12$ & $6.3 \mathrm{E}-12$ & $6.3 \mathrm{E}-12$ & 6. $3 \mathrm{E}-12$ \\
\hline W & $6.3 \mathrm{E}-12$ & $6.3 \mathrm{E}-12$ & $6.3 \mathrm{E}-12$ & $6.3 \mathrm{E}-12$ & $6.3 \mathrm{E}-12$ & $6.3 \mathrm{E}-12$ & $6.3 \mathrm{E}-12$ \\
\hline WSW & $6.3 \mathrm{E}-12$ & $6.3 E-12$ & $6.3 \mathrm{E}-12$ & $6.3 \mathrm{E}-12$ & $6.3 \mathrm{E}-12$ & $6.3 \mathrm{E}-12$ & $6.3 \mathrm{E}-12$ \\
\hline SW & $6.3 \mathrm{E}-12$ & $6.3 E-12$ & $6.3 \mathrm{E}-12$ & $6.3 \mathrm{E}-12$ & $6.3 \mathrm{E}-12$ & $6.3 \mathrm{E}-12$ & $6.3 \mathrm{E}-12$ \\
\hline SSW & $6.3 \mathrm{E}-12$ & $6.3 E-12$ & $6.3 \mathrm{E}-12$ & $6.3 \mathrm{E}-12$ & $6.3 \mathrm{E}-12$ & $6.3 \mathrm{E}-12$ & $6.3 \mathrm{E}-12$ \\
\hline $\mathrm{S}$ & $6.3 E-12$ & $6.3 \mathrm{E}-12$ & $6.3 E-12$ & $6.3 E-12$ & $6.3 E-12$ & $6.3 \mathrm{E}-12$ & $6.3 \mathrm{E}-12$ \\
\hline SSE & $5.0 \mathrm{E}-03$ & $2.1 \mathrm{E}-03$ & $1.9 \mathrm{E}-03$ & $1.9 \mathrm{E}-03$ & $1.8 \mathrm{E}-03$ & $1.7 \mathrm{E}-03$ & $1.7 \mathrm{E}-03$ \\
\hline SE & $6.3 \mathrm{E}-12$ & $6.3 \mathrm{E}-12$ & $6.3 \mathrm{E}-12$ & $6.3 \mathrm{E}-12$ & $6.3 \mathrm{E}-12$ & $6.3 \mathrm{E}-12$ & $6.3 \mathrm{E}-12$ \\
\hline ESE & $6.3 \mathrm{E}-12$ & $6.3 \mathrm{E}-12$ & $6.3 E-12$ & $6.3 \mathrm{E}-12$ & $6.3 \mathrm{E}-12$ & $6.3 \mathrm{E}-12$ & $6.3 \mathrm{E}-12$ \\
\hline E & $6.3 \mathrm{E}-12$ & $6.3 \mathrm{E}-12$ & $6.3 \mathrm{E}-12$ & $6.3 \mathrm{E}-12$ & $6.3 \mathrm{E}-12$ & $6.3 \mathrm{E}-12$ & 6. $3 \mathrm{E}-12$ \\
\hline ENE & $6.3 \mathrm{E}-12$ & $6.3 E-12$ & $6.3 \mathrm{E}-12$ & $6.3 \mathrm{E}-12$ & $6.3 \mathrm{E}-12$ & $6.3 \mathrm{E}-12$ & $6.3 \mathrm{E}-12$ \\
\hline NE & $6.3 E-12$ & $6.3 E-12$ & $6.3 \mathrm{E}-12$ & $6.3 \mathrm{E}-12$ & $6.3 E-12$ & $6.3 E-12$ & $6.3 E-12$ \\
\hline NNE & $6.3 E-12$ & $6.3 E-12$ & $6.3 \mathrm{E}-12$ & $6.3 \mathrm{E}-12$ & $6.3 \mathrm{E}-12$ & $6.3 \mathrm{E}-12$ & $6.3 \mathrm{E}-12$ \\
\hline
\end{tabular}

Distance $(\mathrm{m})$

\begin{tabular}{|c|c|c|c|c|c|c|c|}
\hline Direction & 58780 & 59110 & 61710 & 63600 & 67530 & 69590 & 70340 \\
\hline $\mathrm{N}$ & $6.3 \mathrm{E}-12$ & $6.3 E-12$ & $6.3 E-12$ & $6.3 E-12$ & $6.3 \mathrm{E}-12$ & $6.3 \mathrm{E}-12$ & 6. $3 E-12$ \\
\hline NNW & $6.3 \mathrm{E}-12$ & $6.3 \mathrm{E}-12$ & $6.3 \mathrm{E}-12$ & $6.3 \mathrm{E}-12$ & $6.3 \mathrm{E}-12$ & $6.3 \mathrm{E}-12$ & $6.3 \mathrm{E}-12$ \\
\hline NW & $6.3 \mathrm{E}-12$ & $6.3 \mathrm{E}-12$ & $6.3 \mathrm{E}-12$ & $6.3 \mathrm{E}-12$ & $6.3 \mathrm{E}-12$ & $6.3 \mathrm{E}-12$ & $6.3 \mathrm{E}-12$ \\
\hline WNW & $6.3 \mathrm{E}-12$ & $6.3 E-12$ & $6.3 E-12$ & $6.3 E-12$ & $6.3 \mathrm{E}-12$ & $6.3 \mathrm{E}-12$ & 6. $3 E-12$ \\
\hline W & $6.3 \mathrm{E}-12$ & $6.3 \mathrm{E}-12$ & $6.3 \mathrm{E}-12$ & $6.3 \mathrm{E}-12$ & 6. $3 \mathrm{E}-12$ & $6.3 \mathrm{E}-12$ & 6. $3 \mathrm{E}-12$ \\
\hline WSW & $6.3 \mathrm{E}-12$ & $6.3 \mathrm{E}-12$ & $6.3 \mathrm{E}-12$ & $6.3 \mathrm{E}-12$ & 6. $3 \mathrm{E}-12$ & $6.3 \mathrm{E}-12$ & $6.3 \mathrm{E}-12$ \\
\hline SW & $6.3 E-12$ & $6.3 E-12$ & $6.3 E-12$ & $6.3 \mathrm{E}-12$ & $6.3 \mathrm{E}-12$ & $6.3 \mathrm{E}-12$ & 6. $3 E-12$ \\
\hline SSW & $6.3 \mathrm{E}-12$ & 6. $3 \mathrm{E}-12$ & $6.3 \mathrm{E}-12$ & $6.3 \mathrm{E}-12$ & $6.3 \mathrm{E}-12$ & $6.3 \mathrm{E}-12$ & 6. $3 \mathrm{E}-12$ \\
\hline $\mathrm{S}$ & $6.3 \mathrm{E}-12$ & $6.3 \mathrm{E}-12$ & $6.3 \mathrm{E}-12$ & $6.3 \mathrm{E}-12$ & 6. $3 \mathrm{E}-12$ & $6.3 \mathrm{E}-12$ & $6.3 \mathrm{E}-12$ \\
\hline SSE & $1.5 \mathrm{E}-03$ & 1. 4E- -3 & 1. 3E- - 3 & 1. 3E-०3 & 1. $2 \mathrm{E}-\odot 3$ & 1.1E- -3 & $1.1 \mathrm{E}-03$ \\
\hline SE & $6.3 \mathrm{E}-12$ & $6.3 \mathrm{E}-12$ & $6.3 \mathrm{E}-12$ & $6.3 \mathrm{E}-12$ & $6.3 \mathrm{E}-12$ & $6.3 \mathrm{E}-12$ & $6.3 \mathrm{E}-12$ \\
\hline ESE & $6.3 \mathrm{E}-12$ & $6.3 \mathrm{E}-12$ & $6.3 \mathrm{E}-12$ & $6.3 E-12$ & $6.3 \mathrm{E}-12$ & $6.3 \mathrm{E}-12$ & $6.3 \mathrm{E}-12$ \\
\hline E & $6.3 E-12$ & $6.3 \mathrm{E}-12$ & $6.3 \mathrm{E}-12$ & $6.3 E-12$ & $6.3 \mathrm{E}-12$ & $6.3 \mathrm{E}-12$ & $6.3 \mathrm{E}-12$ \\
\hline ENE & $6.3 \mathrm{E}-12$ & $6.3 \mathrm{E}-12$ & $6.3 \mathrm{E}-12$ & $6.3 \mathrm{E}-12$ & $6.3 \mathrm{E}-12$ & $6.3 \mathrm{E}-12$ & $6.3 \mathrm{E}-12$ \\
\hline NE & $6.3 E-12$ & $6.3 \mathrm{E}-12$ & $6.3 \mathrm{E}-12$ & $6.3 \mathrm{E}-12$ & $6.3 \mathrm{E}-12$ & $6.3 \mathrm{E}-12$ & 6. $3 E-12$ \\
\hline NNE & $6.3 \mathrm{E}-12$ & $6.3 \mathrm{E}-12$ & $6.3 \mathrm{E}-12$ & $6.3 \mathrm{E}-12$ & 6. $3 \mathrm{E}-12$ & $6.3 \mathrm{E}-12$ & 6. $3 \mathrm{E}-12$ \\
\hline
\end{tabular}


Oct 26, $2006 \quad 08: 38$ am

SUMMARY

Page 6

INDIVIDUAL EFFECTIVE DOSE EQUIVALENT RATE (mrem/y)

(All Radionuclides and Pathways)

\begin{tabular}{|c|c|c|c|c|c|c|}
\hline \multirow[b]{2}{*}{ Direction } & \multicolumn{6}{|c|}{ Distance $(\mathrm{m})$} \\
\hline & 73520 & 75750 & 76380 & 78440 & 79130 & 79450 \\
\hline $\mathrm{N}$ & $6.3 \mathrm{E}-12$ & $6.3 \mathrm{E}-12$ & $6.3 \mathrm{E}-12$ & $6.3 \mathrm{E}-12$ & $6.3 \mathrm{E}-12$ & $6.3 \mathrm{E}-12$ \\
\hline NNW & $6.3 \mathrm{E}-12$ & $6.3 E-12$ & $6.3 E-12$ & $6.3 \mathrm{E}-12$ & $6.3 \mathrm{E}-12$ & $6.3 \mathrm{E}-12$ \\
\hline NW & $6.3 E-12$ & $6.3 E-12$ & $6 \cdot 3 E-12$ & $6.3 E-12$ & $6.3 E-12$ & $6.3 E-12$ \\
\hline WNW & $6.3 \mathrm{E}-12$ & $6.3 E-12$ & $6.3 \mathrm{E}-12$ & $6.3 \mathrm{E}-12$ & $6.3 \mathrm{E}-12$ & $6.3 \mathrm{E}-12$ \\
\hline W & $6 \cdot 3 E-12$ & $6 \cdot 3 E-12$ & $6 \cdot 3 E-12$ & $6 \cdot 3 E-12$ & $6 \cdot 3 \mathrm{E}-12$ & $6.3 E-12$ \\
\hline WSW & $6.3 E-12$ & $6 \cdot 3 E-12$ & $6 \cdot 3 E-12$ & $6.3 E-12$ & $6.3 E-12$ & $6.3 E-12$ \\
\hline SW & $6.3 \mathrm{E}-12$ & $6.3 E-12$ & $6.3 \mathrm{E}-12$ & $6.3 \mathrm{E}-12$ & $6.3 \mathrm{E}-12$ & 6. $3 E-12$ \\
\hline SSW & $6.3 \mathrm{E}-12$ & $6.3 \mathrm{E}-12$ & $6.3 \mathrm{E}-12$ & $6.3 \mathrm{E}-12$ & $6.3 E-12$ & $6.3 E-12$ \\
\hline$S$ & $6 \cdot 3 E-12$ & $6 \cdot 3 E-12$ & $6 \cdot 3 E-12$ & $6.3 E-12$ & $6 \cdot 3 E-12$ & $6.3 E-12$ \\
\hline SSE & $1.1 \mathrm{E}-03$ & 1. $\odot E-\odot 3$ & 1. ๑E-๑3 & $9.7 \mathrm{E}-\odot 4$ & $9.6 \mathrm{E}-\odot 4$ & $9.6 \mathrm{E}-04$ \\
\hline SE & $6.3 \mathrm{E}-12$ & $6.3 \mathrm{E}-12$ & $6.3 \mathrm{E}-12$ & $6.3 \mathrm{E}-12$ & $6.3 \mathrm{E}-12$ & $6.3 \mathrm{E}-12$ \\
\hline ESE & $6 \cdot 3 E-12$ & $6 \cdot 3 E-12$ & $6 \cdot 3 E-12$ & $6 \cdot 3 E-12$ & $6 \cdot 3 E-12$ & $6.3 E-12$ \\
\hline E & $6.3 E-12$ & $6.3 E-12$ & $6.3 E-12$ & $6.3 E-12$ & $6.3 E-12$ & $6.3 \mathrm{E}-12$ \\
\hline ENE & $6.3 E-12$ & $6.3 E-12$ & $6.3 E-12$ & $6.3 E-12$ & $6.3 E-12$ & $6.3 E-12$ \\
\hline $\mathrm{NE}$ & $6.3 E-12$ & $6.3 E-12$ & $6.3 \mathrm{E}-12$ & $6.3 \mathrm{E}-12$ & $6.3 \mathrm{E}-12$ & $6.3 \mathrm{E}-12$ \\
\hline NNE & $6 \cdot 3 E-12$ & $6 \cdot 3 E-12$ & $6 \cdot 3 E-12$ & $6.3 E-12$ & $6 \cdot 3 E-12$ & $6.3 E-12$ \\
\hline
\end{tabular}


Oct 26, $2006 \quad 08: 38$ am

SUMMARY

Page 7

INDIVIDUAL LIFETIME RISK (deaths)

(All Radionuclides and Pathways)

\begin{tabular}{|c|c|c|c|c|c|c|c|}
\hline \multirow[b]{2}{*}{ Direction } & \multicolumn{6}{|c|}{ Distance (m) } & \multirow[b]{2}{*}{54360} \\
\hline & 22600 & 46710 & 48940 & 49330 & 51120 & 53270 & \\
\hline $\mathrm{N}$ & 4. $. \mathrm{E}-18$ & 4. $0 \mathrm{E}-18$ & 4. $0 \mathrm{E}-18$ & 4. $0 \mathrm{E}-18$ & 4. $0 \mathrm{E}-18$ & 4. $. \mathrm{E}-18$ & 4. $0 \mathrm{E}-18$ \\
\hline NNW & $4.0 \mathrm{E}-18$ & 4. $0 \mathrm{E}-18$ & 4. $. \mathrm{E}-18$ & 4. $0 \mathrm{E}-18$ & 4. $0 \mathrm{E}-18$ & 4. $0 \mathrm{E}-18$ & 4. $0 \mathrm{E}-18$ \\
\hline NW & 4. $\odot E-18$ & 4. $\odot E-18$ & 4. $0 \mathrm{E}-18$ & 4. $\odot E-18$ & 4. $0 \mathrm{E}-18$ & 4. $\odot E-18$ & 4. $0 \mathrm{E}-18$ \\
\hline WNW & 4. $0 \mathrm{E}-18$ & 4. $\odot E-18$ & 4. $0 \mathrm{E}-18$ & 4. $0 \mathrm{E}-18$ & $4.0 \mathrm{E}-18$ & $4.0 \mathrm{E}-18$ & 4. $0 \mathrm{E}-18$ \\
\hline W & $4.0 \mathrm{E}-18$ & 4. $0 \mathrm{E}-18$ & $4.0 \mathrm{E}-18$ & 4. $0 \mathrm{E}-18$ & $4.0 \mathrm{E}-18$ & $4.0 \mathrm{E}-18$ & 4. $0 \mathrm{E}-18$ \\
\hline WSW & 4. $\odot E-18$ & 4. $\odot E-18$ & 4. $0 \mathrm{E}-18$ & 4. $\odot E-18$ & 4. $0 \mathrm{E}-18$ & 4. $\odot E-18$ & 4. $0 \mathrm{E}-18$ \\
\hline SW & 4. $0 \mathrm{E}-18$ & 4. $0 \mathrm{E}-18$ & 4. $. \mathrm{E}-18$ & 4. . $0 \mathrm{E}-18$ & 4. $0 \mathrm{E}-18$ & 4. $0 \mathrm{E}-18$ & 4. $0 \mathrm{E}-18$ \\
\hline SSW & $4.0 E-18$ & 4. $0 \mathrm{E}-18$ & $4.0 \mathrm{E}-18$ & $4.0 \mathrm{E}-18$ & $4.0 \mathrm{E}-18$ & $4.0 \mathrm{E}-18$ & $4.0 \mathrm{E}-18$ \\
\hline $\mathrm{S}$ & 4. $\odot E-18$ & 4. $\odot E-18$ & 4. $\odot \mathrm{E}-18$ & 4. $0 \mathrm{E}-18$ & 4. $0 \mathrm{E}-18$ & 4. $\odot E-18$ & 4. $0 \mathrm{E}-18$ \\
\hline SSE & $1.6 \mathrm{E}-09$ & $6.7 \mathrm{E}-10$ & $6.3 E-10$ & $6.2 \mathrm{E}-10$ & $5.9 \mathrm{E}-10$ & $5.6 \mathrm{E}-10$ & $5.4 \mathrm{E}-10$ \\
\hline SE & 4. $0 \mathrm{E}-18$ & 4. $0 \mathrm{E}-18$ & 4. $\odot \mathrm{E}-18$ & 4. $0 \mathrm{E}-18$ & 4. $0 \mathrm{E}-18$ & 4. $0 \mathrm{E}-18$ & 4. $0 \mathrm{E}-18$ \\
\hline ESE & 4. $0 \mathrm{E}-18$ & 4. $0 \mathrm{E}-18$ & 4. $\odot \mathrm{E}-18$ & 4. $\odot E-18$ & 4. $0 \mathrm{E}-18$ & 4. $0 \mathrm{E}-18$ & 4. $0 \mathrm{E}-18$ \\
\hline E & $4.0 E-18$ & 4. $0 \mathrm{E}-18$ & 4. $0 \mathrm{E}-18$ & 4. $0 \mathrm{E}-18$ & $4.0 E-18$ & $4.0 E-18$ & 4. $0 \mathrm{E}-18$ \\
\hline ENE & 4. $0 \mathrm{E}-18$ & 4. $0 \mathrm{E}-18$ & 4. $\odot \mathrm{E}-18$ & 4. $\odot \mathrm{E}-18$ & 4. $0 \mathrm{E}-18$ & 4. $\odot E-18$ & 4. $0 \mathrm{E}-18$ \\
\hline NE & 4. $\odot E-18$ & 4. $0 \mathrm{E}-18$ & 4. $\odot \mathrm{E}-18$ & 4. $\odot \mathrm{E}-18$ & 4. $0 \mathrm{E}-18$ & 4. $\odot E-18$ & 4. $0 \mathrm{E}-18$ \\
\hline NNE & 4. $0 \mathrm{E}-18$ & 4. $0 \mathrm{E}-18$ & 4. $0 \mathrm{E}-18$ & 4. $0 \mathrm{E}-18$ & 4. $0 \mathrm{E}-18$ & 4. $0 \mathrm{E}-18$ & $4.0 \mathrm{E}-18$ \\
\hline
\end{tabular}

Distance $(\mathrm{m})$

\begin{tabular}{|c|c|c|c|c|c|c|c|}
\hline Direction & 58780 & 59110 & 61710 & 63600 & 67530 & 69590 & 70340 \\
\hline $\mathrm{N}$ & 4. $0 E-18$ & 4. $0 \mathrm{E}-18$ & $4.0 \mathrm{E}-18$ & 4. $0 \mathrm{E}-18$ & $4.0 \mathrm{E}-18$ & 4. $0 \mathrm{E}-18$ & $4.0 \mathrm{E}-18$ \\
\hline NNW & 4. $\odot E-18$ & 4. $\odot E-18$ & 4. $0 \mathrm{E}-18$ & 4. $0 \mathrm{E}-18$ & 4. $0 \mathrm{E}-18$ & 4. $\odot E-18$ & 4. $0 \mathrm{E}-18$ \\
\hline NW & 4. $0 \mathrm{E}-18$ & 4. $0 \mathrm{E}-18$ & 4. $0 \mathrm{E}-18$ & 4. $0 \mathrm{E}-18$ & 4. $0 \mathrm{E}-18$ & 4. $0 \mathrm{E}-18$ & 4. $0 \mathrm{E}-18$ \\
\hline WNW & 4. $0 \mathrm{E}-18$ & 4. $0 \mathrm{E}-18$ & 4. $0 \mathrm{E}-18$ & 4. $0 \mathrm{E}-18$ & 4. $0 \mathrm{E}-18$ & 4. $0 \mathrm{E}-18$ & $4.0 \mathrm{E}-18$ \\
\hline W & 4. $\odot E-18$ & 4. $\odot E-18$ & 4. $0 \mathrm{E}-18$ & 4. $0 \mathrm{E}-18$ & 4. $0 \mathrm{E}-18$ & 4. $\odot E-18$ & 4. $0 \mathrm{E}-18$ \\
\hline WSW & 4. $0 \mathrm{E}-18$ & 4. $0 \mathrm{E}-18$ & 4. $0 \mathrm{E}-18$ & 4. $0 \mathrm{E}-18$ & 4. $0 \mathrm{E}-18$ & 4. $0 \mathrm{E}-18$ & 4. $0 \mathrm{E}-18$ \\
\hline SW & $4.0 \mathrm{E}-18$ & 4. $0 \mathrm{E}-18$ & $4.0 \mathrm{E}-18$ & $4.0 \mathrm{E}-18$ & $4.0 \mathrm{E}-18$ & 4. $0 \mathrm{E}-18$ & $4.0 \mathrm{E}-18$ \\
\hline SSW & 4. $0 \mathrm{E}-18$ & 4. $0 \mathrm{E}-18$ & 4. $0 \mathrm{E}-18$ & 4. $0 \mathrm{E}-18$ & 4. $0 \mathrm{E}-18$ & 4. $\odot E-18$ & 4. $0 \mathrm{E}-18$ \\
\hline$S$ & $4.0 \mathrm{E}-18$ & $4.0 \mathrm{E}-18$ & 4. $0 \mathrm{E}-18$ & 4. $0 \mathrm{E}-18$ & 4. $0 \mathrm{E}-18$ & 4. $0 \mathrm{E}-18$ & $4.0 \mathrm{E}-18$ \\
\hline SSE & $4.7 \mathrm{E}-10$ & $4.6 \mathrm{E}-10$ & $4.3 E-10$ & $4.2 \mathrm{E}-10$ & $3.9 \mathrm{E}-10$ & $3.7 \mathrm{E}-10$ & $3.7 \mathrm{E}-10$ \\
\hline SE & 4. $0 \mathrm{E}-18$ & 4. $0 \mathrm{E}-18$ & 4. $0 \mathrm{E}-18$ & 4. $0 \mathrm{E}-18$ & 4. $0 \mathrm{E}-18$ & 4. $0 \mathrm{E}-18$ & 4. $0 \mathrm{E}-18$ \\
\hline ESE & $4.0 \mathrm{E}-18$ & 4. $0 \mathrm{E}-18$ & $4.0 E-18$ & 4. $0 \mathrm{E}-18$ & $4.0 \mathrm{E}-18$ & 4. $0 \mathrm{E}-18$ & 4. $0 \mathrm{E}-18$ \\
\hline $\mathrm{E}$ & 4. $0 \mathrm{E}-18$ & $4.0 \mathrm{E}-18$ & 4. $0 \mathrm{E}-18$ & 4. $0 \mathrm{E}-18$ & 4. $0 \mathrm{E}-18$ & 4. $0 \mathrm{E}-18$ & 4. $0 \mathrm{E}-18$ \\
\hline ENE & 4. $0 \mathrm{E}-18$ & 4. $0 \mathrm{E}-18$ & 4. $0 \mathrm{E}-18$ & 4. $0 \mathrm{E}-18$ & 4. $0 \mathrm{E}-18$ & 4. $0 \mathrm{E}-18$ & 4. $0 \mathrm{E}-18$ \\
\hline NE & $4.0 \mathrm{E}-18$ & 4. $0 \mathrm{E}-18$ & $4.0 \mathrm{E}-18$ & 4. $0 \mathrm{E}-18$ & 4. $0 \mathrm{E}-18$ & 4. $0 \mathrm{E}-18$ & $4.0 \mathrm{E}-18$ \\
\hline NNE & 4. $0 \mathrm{E}-18$ & 4. $0 \mathrm{E}-18$ & 4. $0 \mathrm{E}-18$ & 4. $0 \mathrm{E}-18$ & 4. $0 \mathrm{E}-18$ & 4. $0 \mathrm{E}-18$ & 4. $0 \mathrm{E}-18$ \\
\hline
\end{tabular}


oct 26, 2006 08:38 am

SUMMARY

Page 8

INDIVIDUAL LIFETIME RISK (deaths)

(All Radionuclides and Pathways)

\begin{tabular}{|c|c|c|c|c|c|c|}
\hline \multirow[b]{2}{*}{ Direction } & \multicolumn{6}{|c|}{ Distance $(\mathrm{m})$} \\
\hline & 73520 & 75750 & 76380 & 78440 & 79130 & 79450 \\
\hline $\mathrm{N}$ & 4. $0 \mathrm{E}-18$ & 4. $0 \mathrm{E}-18$ & 4. $0 \mathrm{E}-18$ & 4. $0 \mathrm{E}-18$ & 4. $0 \mathrm{E}-18$ & 4. $0 \mathrm{E}-18$ \\
\hline NNW & 4. $0 \mathrm{E}-18$ & 4. $0 \mathrm{E}-18$ & 4. $0 \mathrm{E}-18$ & 4. $0 \mathrm{E}-18$ & 4. $0 \mathrm{E}-18$ & 4. $0 \mathrm{E}-18$ \\
\hline NW & 4. $. \mathrm{E}-18$ & 4. $0 \mathrm{E}-18$ & 4. $. E-18$ & 4. $0 \mathrm{E}-18$ & 4. $0 \mathrm{E}-18$ & 4. $0 \mathrm{E}-18$ \\
\hline WNW & 4. $\odot E-18$ & 4. $0 \mathrm{E}-18$ & 4. $0 \mathrm{E}-18$ & 4. $0 \mathrm{E}-18$ & 4. $0 \mathrm{E}-18$ & 4. $0 \mathrm{E}-18$ \\
\hline W & 4. $0 \mathrm{E}-18$ & 4. $0 \mathrm{E}-18$ & 4. $0 \mathrm{E}-18$ & 4. $0 \mathrm{E}-18$ & 4. $0 \mathrm{E}-18$ & $4.0 \mathrm{E}-18$ \\
\hline WSW & 4. $\odot E-18$ & 4. $0 \mathrm{E}-18$ & 4. $\odot E-18$ & 4. $0 \mathrm{E}-18$ & 4. $0 \mathrm{E}-18$ & $4.0 E-18$ \\
\hline SW & 4. $\odot E-18$ & 4. $\odot E-18$ & 4. $\odot \mathrm{E}-18$ & 4. $\odot E-18$ & 4. $0 \mathrm{E}-18$ & 4. $0 \mathrm{E}-18$ \\
\hline SSW & 4. $0 \mathrm{E}-18$ & 4. $0 \mathrm{E}-18$ & 4. $\mathrm{OE}-18$ & 4. $0 \mathrm{E}-18$ & 4. $0 \mathrm{E}-18$ & $4.0 \mathrm{E}-18$ \\
\hline $\mathrm{S}$ & 4. $0 \mathrm{E}-18$ & 4. $0 \mathrm{E}-18$ & 4. $0 \mathrm{E}-18$ & 4. $0 \mathrm{E}-18$ & $4.0 \mathrm{E}-18$ & 4. $\mathrm{OE}-18$ \\
\hline SSE & $3.4 \mathrm{E}-10$ & 3. $3 E-10$ & $3.3 E-10$ & $3.2 \mathrm{E}-10$ & $3.1 \mathrm{E}-10$ & $3.1 \mathrm{E}-10$ \\
\hline SE & 4. $0 \mathrm{E}-18$ & 4. $0 \mathrm{E}-18$ & 4. $0 \mathrm{E}-18$ & 4. $0 \mathrm{E}-18$ & 4. $0 \mathrm{E}-18$ & 4. $0 \mathrm{E}-18$ \\
\hline ESE & 4. $0 \mathrm{E}-18$ & $4.0 \mathrm{E}-18$ & 4. $0 \mathrm{E}-18$ & 4. $0 \mathrm{E}-18$ & $4.0 \mathrm{E}-18$ & 4. $0 \mathrm{E}-18$ \\
\hline$E$ & 4. $\odot E-18$ & 4. $\odot E-18$ & 4. $\odot E-18$ & 4. $\odot E-18$ & 4. $\odot E-18$ & 4. $0 \mathrm{E}-18$ \\
\hline ENE & 4. $0 \mathrm{E}-18$ & 4. $0 \mathrm{E}-18$ & 4. $0 \mathrm{E}-18$ & 4. $0 \mathrm{E}-18$ & 4. $0 \mathrm{E}-18$ & 4. $0 \mathrm{E}-18$ \\
\hline NE & 4. $0 \mathrm{E}-18$ & 4. $0 \mathrm{E}-18$ & 4. $0 \mathrm{E}-18$ & 4. $0 \mathrm{E}-18$ & $4.0 \mathrm{E}-18$ & 4. $0 \mathrm{E}-18$ \\
\hline NNE & $4.0 \mathrm{E}-18$ & 4. $. E-18$ & 4. $. E-18$ & 4. $0 \mathrm{E}-18$ & 4. $0 \mathrm{E}-18$ & 4. $0 \mathrm{E}-18$ \\
\hline
\end{tabular}




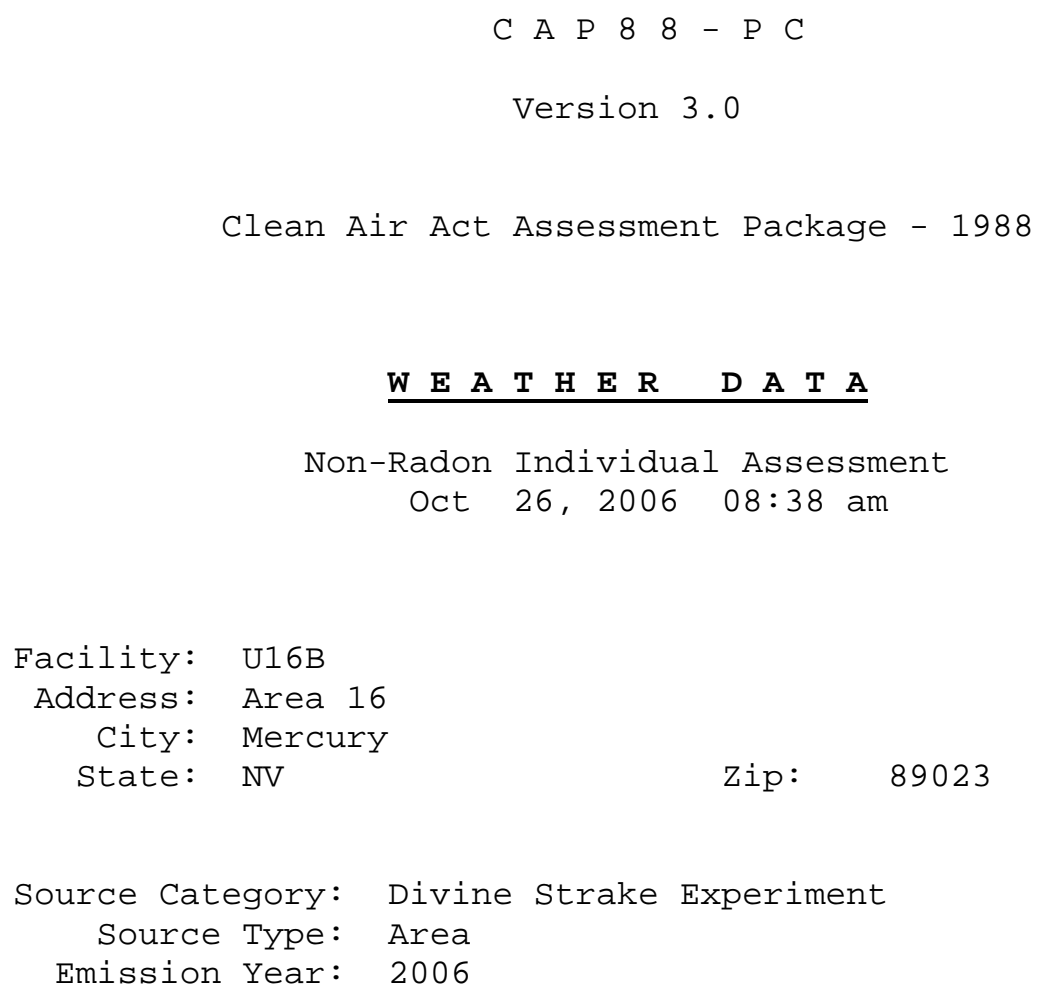

Comments: Dose from the proposed Divine Strake experiment: Single wind direction used. Source used was maximum upper confidence interval reported in DOE/NV-1177 and total PM10.

Dataset Name: DS SNJV Max UCI

Dataset Date: 10/26/2006 7:32:00 AM

Wind File: C:\Program Files \CAP88-PC30\WindLib\Divine 
HARMONIC AVERAGE WIND SPEEDS (WIND TOWARDS)

\begin{tabular}{|c|c|c|c|c|c|c|c|c|}
\hline \multirow[b]{2}{*}{ Dir } & \multicolumn{7}{|c|}{ Pasquill Stability Class } & \multirow[b]{2}{*}{$\begin{array}{l}\text { Wind } \\
\text { Freq }\end{array}$} \\
\hline & $A$ & $B$ & C & $\mathrm{D}$ & $E$ & $F$ & G & \\
\hline $\mathrm{N}$ & 0.000 & 0.000 & 0.000 & 0.000 & 0.000 & 0.000 & 0.000 & 0.000 \\
\hline NNW & 0.000 & 0.000 & 0.000 & 0.000 & 0.000 & 0.000 & 0.000 & 0.000 \\
\hline NW & 0.000 & 0.000 & 0.000 & 0.000 & 0.000 & 0.000 & 0.000 & 0.000 \\
\hline WNW & $\odot . \odot \odot \odot$ & $\odot .0 \odot \odot$ & $\odot .0 \odot \odot$ & $\odot . \odot \odot \odot$ & $\odot . \odot \odot \odot$ & $\odot .0 \odot \odot$ & $\odot .0 \odot \odot$ & $\odot .00 \odot$ \\
\hline W & 0.000 & 0.000 & 0.000 & 0.000 & 0.000 & 0.000 & 0.000 & 0.000 \\
\hline WSW & 0.000 & 0.000 & 0.000 & 0.000 & 0.000 & 0.000 & 0.000 & 0.000 \\
\hline SW & $\odot .0 \odot \odot$ & $\odot . \odot \odot \odot$ & $\odot .0 \odot \odot$ & $\odot . \odot \odot \odot$ & $\odot . \odot \odot \odot$ & $\odot .0 \odot \odot$ & $\odot .0 \odot \odot$ & $\odot .00 \odot$ \\
\hline SSW & $\odot .0 \odot \odot$ & $\odot .0 \odot \odot$ & $\odot .0 \odot \odot$ & $\odot . \odot \odot \odot$ & $\odot . \odot \odot \odot$ & $\odot .0 \odot \odot$ & $\odot .0 \odot \odot$ & $\odot .00 \odot$ \\
\hline S & 0.000 & 0.000 & 0.000 & 0.000 & 0.000 & 0.000 & 0.000 & 0.000 \\
\hline SSE & $\odot . \odot \odot \odot$ & $\odot . \odot \odot \odot$ & $\odot .0 \odot \odot$ & $\odot . \odot \odot \odot$ & 3.089 & $\odot .0 \odot \odot$ & $\odot .0 \odot \odot$ & 1.000 \\
\hline SE & $\odot .0 \odot \odot$ & $\odot .0 \odot \odot$ & $\odot .0 \odot \odot$ & $\odot . \odot \odot \odot$ & $\odot . \odot \odot \odot$ & $\odot .00 \odot$ & $0.0 \odot \odot$ & $\odot .00 \odot$ \\
\hline ESE & $\odot .000$ & $\odot .000$ & 0.000 & $\odot .0 \odot \odot$ & $\odot .00 \%$ & $\odot .000$ & $\odot .000$ & $\odot .000$ \\
\hline$E$ & $\odot .0 \odot \odot$ & 0.000 & 0.000 & ๑. . 000 & $\odot .000$ & 0.000 & $\odot .000$ & 0.000 \\
\hline ENE & $\odot .0 \odot ०$ & $\odot .000$ & 0.000 & $\odot .0 \odot \odot$ & $\odot .0 \odot ०$ & $\odot .000$ & 0.000 & $\odot .00 \odot$ \\
\hline NE & 0.000 & $\odot .000$ & 0.000 & $\odot .0 \odot ०$ & $\odot .000$ & $\odot .000$ & 0.000 & 0.000 \\
\hline NNE & 0.000 & 0.000 & 0.000 & 0.000 & 0.000 & 0.000 & 0.000 & 0.000 \\
\hline
\end{tabular}

ARITHMETIC AVERAGE WIND SPEEDS (WIND TOWARDS)

Pasquill Stability Class

\begin{tabular}{|c|c|c|c|c|c|c|c|}
\hline Dir & $A$ & B & C & D & E & $F$ & G \\
\hline $\mathrm{N}$ & 0.000 & 0.000 & 0.000 & $0.0 \odot 0$ & 0.000 & 0.000 & $\odot .000$ \\
\hline NNW & 0.000 & 0.000 & 0.000 & 0.000 & 0.000 & 0.000 & 0.000 \\
\hline NW & 0.000 & 0.000 & 0.000 & 0.000 & 0.000 & 0.000 & 0.000 \\
\hline WNW & 0.000 & 0.000 & 0.000 & 0.000 & $\odot .000$ & 0.000 & 0.000 \\
\hline W & 0.000 & 0.000 & 0.000 & 0.000 & 0.000 & 0.000 & 0.000 \\
\hline WSW & 0.000 & 0.000 & 0.000 & 0.000 & $\odot .000$ & 0.000 & 0.000 \\
\hline SW & 0.000 & 0.000 & 0.000 & 0.000 & 0.000 & 0.000 & 0.000 \\
\hline SSW & $\odot .0 \odot \odot$ & $\odot .000$ & 0.000 & 0.000 & 0.000 & $\odot .0 \odot \odot$ & $\odot .00 \odot$ \\
\hline S & 0.000 & 0.000 & 0.000 & 0.000 & 0.000 & 0.000 & 0.000 \\
\hline SSE & 0.000 & 0.000 & $\odot .0 \odot \odot$ & 0.000 & 3.089 & $\odot .000$ & $\odot .000$ \\
\hline SE & 0.000 & 0.000 & 0.000 & 0.000 & 0.000 & 0.000 & 0.000 \\
\hline ESE & 0.000 & $\odot .000$ & $\odot .0 \odot \odot$ & 0.000 & 0.000 & 0.000 & $\odot .000$ \\
\hline$E$ & 0.000 & 0.000 & 0.000 & 0.000 & 0.000 & 0.000 & 0.000 \\
\hline ENE & $\odot .000$ & $\odot .000$ & $\odot .0 \odot \odot$ & 0.000 & $\odot .000$ & $\odot .000$ & $\odot .000$ \\
\hline NE & 0.000 & 0.000 & 0.000 & 0.000 & 0.000 & 0.000 & 0.000 \\
\hline NNE & 0.000 & $\odot .0 \odot \odot$ & 0.000 & 0.000 & 0.000 & 0.000 & $\odot .000$ \\
\hline
\end{tabular}


FREQUENCIES OF STABILITY CLASSES (WIND TOWARDS)

\begin{tabular}{|c|c|c|c|c|c|c|c|}
\hline \multirow[b]{2}{*}{ Dir } & \multicolumn{7}{|c|}{ Pasquill Stability Class } \\
\hline & A & B & C & D & E & $\mathrm{F}$ & G \\
\hline $\mathrm{N}$ & 0.0000 & 0.0000 & 0.0000 & 0.0000 & 0.0000 & $\odot .000 \odot$ & $\odot .000 \odot$ \\
\hline NNW & $\odot . \odot \odot \odot \odot$ & $\odot .0 \odot \odot \odot$ & $\odot .0 \odot \odot \odot$ & $\odot .0 \odot \odot \odot$ & $\odot .0 \odot \odot \odot$ & $\odot .0 \odot \odot \odot$ & $\odot .0 \odot \odot \odot$ \\
\hline NW & $\odot . \odot \odot \odot \odot$ & $\odot . \odot \odot \odot \odot$ & $\odot . \odot \odot \odot \odot$ & $\odot . \odot \odot \odot \odot$ & $\odot . \odot \odot \odot \odot$ & $\odot . \odot \odot \odot \odot$ & $\odot . \odot \odot \odot \odot$ \\
\hline WNW & $\odot .0 \odot \odot \odot$ & $\odot .000 \odot$ & $\odot .0000$ & $\odot . \odot \odot \odot \odot$ & $\odot . \odot \odot \odot \odot$ & $\odot .0 \odot \odot \odot$ & $\odot .0000$ \\
\hline W & $\odot . \odot \odot \odot \odot$ & 0.0000 & $\odot .0 \odot \odot \odot$ & $\odot .0 \odot \odot \odot$ & $0.000 \odot$ & $\odot .0 \odot \odot \odot$ & $\odot .0 \odot \odot \odot$ \\
\hline WSW & $\odot . \odot \odot \odot \odot$ & $\odot . \odot \odot \odot \odot$ & $\odot . \odot \odot \odot \odot$ & $\odot . \odot \odot \odot \odot$ & $\odot . \odot \odot \odot \odot$ & $\odot . \odot \odot \odot \odot$ & $\odot . \odot \odot \odot \odot$ \\
\hline SW & $\odot . \odot \odot \odot \odot$ & $\odot . \odot \odot \odot \odot$ & $\odot .0 \odot \odot \odot$ & $\odot . \odot \odot \odot \odot$ & $\odot .0 \odot \odot \odot$ & $\odot .0 \odot \odot \odot$ & $\odot .0 \odot \odot \odot$ \\
\hline SSW & $\odot . \odot \odot \odot \odot$ & $\odot .0 \odot \odot \odot$ & $\odot . \odot \odot \odot \odot$ & $\odot . \odot \odot \odot \odot$ & $\odot .0 \odot \odot \odot$ & $\odot .0 \odot \odot \odot$ & $\odot .0 \odot \odot \odot$ \\
\hline $\mathrm{S}$ & $\odot . \odot \odot \odot \odot$ & $\odot . \odot \odot \odot \odot$ & $\odot . \odot \odot \odot \odot$ & $\odot . \odot \odot \odot \odot$ & $\odot . \odot \odot \odot \odot$ & $\odot .0 \odot \odot \odot$ & $\odot . \odot \odot \odot \odot$ \\
\hline SSE & 0.0000 & 0.0000 & 0.0000 & 0.0000 & 1.0000 & 0.0000 & 0.0000 \\
\hline SE & 0.0000 & 0.0000 & 0.0000 & 0.0000 & 0.0000 & 0.0000 & 0.0000 \\
\hline ESE & $\odot . \odot \odot \odot \odot$ & $\odot . \odot \odot \odot \odot$ & $\odot . \odot \odot \odot \odot$ & $\odot . \odot \odot \odot \odot$ & $\odot . \odot \odot \odot \odot$ & $\odot . \odot \odot \odot \odot$ & $\odot . \odot \odot \odot \odot$ \\
\hline $\mathrm{E}$ & $\odot .0000$ & 0.0000 & 0.0000 & 0.0000 & 0.0000 & 0.0000 & 0.0000 \\
\hline ENE & $\odot . \odot \odot \odot \odot$ & $\odot .0 \odot \odot \odot$ & $\odot .0 \odot \odot \odot$ & $\odot . \odot \odot \odot \odot$ & $\odot .0 \odot \odot \odot$ & $\odot .0 \odot \odot \odot$ & $\odot .0 \odot \odot \odot$ \\
\hline $\mathrm{NE}$ & $\odot . \odot \odot \odot \odot$ & $\odot . \odot \odot \odot \odot$ & $\odot . \odot \odot \odot \odot$ & $\odot . \odot \odot \odot \odot$ & $\odot . \odot \odot \odot \odot$ & $\odot . \odot \odot \odot \odot$ & $\odot . \odot \odot \odot \odot$ \\
\hline NNE & $\odot . \odot \odot \odot \odot$ & $\odot . \odot \odot \odot \odot$ & $\odot . \odot \odot \odot \odot$ & 0.0000 & 0.0000 & 0.0000 & 0.0000 \\
\hline TOTAL & $\odot .0 \odot \odot \odot$ & $\odot .000 \odot$ & $\odot .00 \odot \odot$ & $\odot .0000$ & 1.0000 & $\odot .000 \odot$ & $\odot .0 \odot 0 \odot$ \\
\hline
\end{tabular}

ADDITIONAL WEATHER INFORMATION

Average Air Temperature: 13.2 degrees $\mathrm{C}$ 286.36 K

Precipitation: $\quad 3.0 \mathrm{~cm} / \mathrm{y}$ Humidity: $\quad 3.0 \mathrm{~g} / \mathrm{cu} \mathrm{m}$

Lid Height: 3000 meters

Surface Roughness Length: 0.010 meters Height of Wind Measurements: 10.0 meters Average Wind Speed: $3.089 \mathrm{~m} / \mathrm{s}$

Vertical Temperature Gradients:

$\begin{array}{ll}\text { STABILITY E } & 0.073 \mathrm{k} / \mathrm{m} \\ \text { STABILITY F } & 0.109 \mathrm{k} / \mathrm{m} \\ \text { STABILITY G } & 0.146 \mathrm{k} / \mathrm{m}\end{array}$




\author{
C A P $88-P$ C \\ Version 3.0 \\ Clean Air Act Assessment Package - 1988 \\ GEN E R A L D A T A \\ Non-Radon Individual Assessment \\ oct 26, 2006 08:38 am

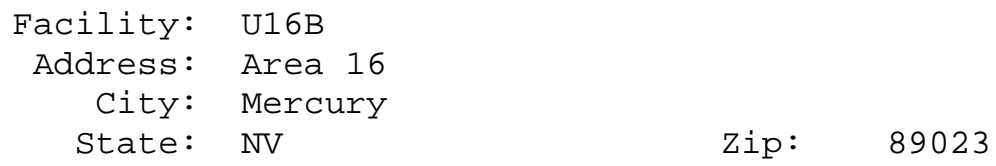

Comments: Dose from the proposed Divine Strake experiment: Single wind direction used. Source used was maximum upper confidence interval reported in D0E/NV-1177 and total PM10.

Dataset Name: DS SNJV Max UCI

Dataset Date: 10/26/2006 7:32:00 AM

Stake \6KNTE. WND 


\begin{tabular}{|c|c|c|c|c|}
\hline Nuclide & $\begin{array}{l}\text { Clearance } \\
\text { Type }\end{array}$ & $\begin{array}{l}\text { Particle } \\
\text { Size } \\
\text { (microns) }\end{array}$ & $\begin{array}{l}\text { Scavenging } \\
\text { Coefficient } \\
\text { (per second) }\end{array}$ & $\begin{array}{c}\text { Dry } \\
\text { Deposition } \\
\text { Velocity } \\
(\mathrm{m} / \mathrm{s})\end{array}$ \\
\hline Cs - 137 & $\mathrm{~F}$ & 1 & $3.00 E-07$ & $1.80 E-03$ \\
\hline $\mathrm{Ba}-137 \mathrm{~m}$ & $M$ & 1 & $3.00 E-07$ & $1.80 E-03$ \\
\hline Am- 241 & $M$ & 1 & $3.00 E-07$ & $1.80 E-03$ \\
\hline$N p-237$ & $M$ & 1 & $3.00 \mathrm{E}-07$ & 1. 80E-०3 \\
\hline $\mathrm{Pa}-233$ & $M$ & 1 & $3.00 E-07$ & $1.80 E-03$ \\
\hline$U-233$ & $M$ & 1 & $3.00 E-07$ & $1.80 E-03$ \\
\hline Th - 229 & $\mathrm{~S}$ & 1 & $3.00 E-07$ & $1.80 E-03$ \\
\hline $\mathrm{Ra}-225$ & $M$ & 1 & $3.00 \mathrm{E}-07$ & $1.80 E-03$ \\
\hline$A c-225$ & $M$ & 1 & $3.00 E-07$ & $1.80 E-03$ \\
\hline Fr - 221 & $M$ & 1 & $3.00 E-\odot 7$ & 1. 80E- - 3 \\
\hline At -217 & $M$ & 1 & $3.00 \mathrm{E}-\odot 7$ & 1. 80E- - 3 \\
\hline $\mathrm{Bi}-213$ & $M$ & 1 & $3.00 \mathrm{E}-07$ & $1.80 E-03$ \\
\hline Po- 213 & $M$ & 1 & $3.00 E-07$ & $1.80 E-03$ \\
\hline$P b-209$ & $M$ & 1 & $3.0 \odot E-\odot 7$ & 1. 80E- - 3 \\
\hline Tl- 209 & $M$ & 1 & $3.00 \mathrm{E}-\odot 7$ & 1. 80E- - 3 \\
\hline Pu- 238 & $M$ & 1 & $3.00 \mathrm{E}-07$ & $1.80 E-03$ \\
\hline U-234 & $M$ & 1 & $3 . \odot \odot E-\odot 7$ & 1. 80E-०3 \\
\hline Th - 230 & $\mathrm{~S}$ & 1 & $3 . \odot \odot E-\odot 7$ & 1. 80E- - 3 \\
\hline $\mathrm{Ra}-226$ & $M$ & 1 & $3.00 E-\odot 7$ & 1. 80E- - 3 \\
\hline Rn - 222 & $\mathrm{G}$ & $\odot$ & $\odot . \odot \odot E+\odot \odot$ & $\odot . \odot \odot E+\odot \odot$ \\
\hline Po- 218 & $M$ & 1 & $3.00 \mathrm{E}-07$ & 1. 80E- - 3 \\
\hline $\mathrm{Pb}-214$ & $M$ & 1 & $3.00 \mathrm{E}-\odot 7$ & 1. 80E- - 3 \\
\hline $\mathrm{Bi}-214$ & $M$ & 1 & 3. ००E - ०7 & 1. 80E- - 33 \\
\hline Po- 214 & $M$ & 1 & 3. ००E - ०७ & $1.80 E-03$ \\
\hline $\mathrm{Pb}-210$ & M & 1 & 3. ००E - ๑7 & 1. 80E- - 3 \\
\hline $\mathrm{Bi}-210$ & $M$ & 1 & 3. ००E - ०7 & 1. 80E- - 3 \\
\hline Po- 210 & M & 1 & $3.0 \odot E-\odot 7$ & 1. 80E-०3 \\
\hline At - 218 & $M$ & 1 & 3. ००E - ०७ & 1. 80E- - 3 \\
\hline Sr-90 & $M$ & 1 & 3. ००E - ๑7 & 1. 8०E-०3 \\
\hline$Y-90$ & $M$ & 1 & 3. ००E - ๑7 & 1. 80E-०3 \\
\hline $\mathrm{K}-40$ & $M$ & 1 & 3. ००E - ०7 & 1. 80E-०3 \\
\hline Th- 232 & $\mathrm{~S}$ & 1 & $3.00 E-07$ & $1.80 E-03$ \\
\hline $\mathrm{Ra}-228$ & $M$ & 1 & $3.00 E-07$ & $1.80 E-03$ \\
\hline$A c-228$ & $M$ & 1 & 3. ००E - ०7 & 1. 80E-०3 \\
\hline Th - 228 & $\mathrm{~S}$ & 1 & $3.00 E-07$ & $1.80 E-03$ \\
\hline $\mathrm{Ra}-224$ & $M$ & 1 & $3.00 E-07$ & $1.80 E-03$ \\
\hline Rn - 220 & G & $\odot$ & $\odot .00 \mathrm{E}+0 \odot$ & $\odot . \odot \odot E+\odot \odot$ \\
\hline Po- 216 & $M$ & 1 & $3.00 E-07$ & $1.80 E-03$ \\
\hline $\mathrm{Pb}-212$ & $M$ & 1 & $3.00 E-07$ & $1.80 E-03$ \\
\hline $\mathrm{Bi}-212$ & $M$ & 1 & 3. ००E - ๑7 & 1. 80E-०3 \\
\hline Po- 212 & $M$ & 1 & $3.00 E-07$ & $1.80 E-03$ \\
\hline Tl- 208 & $M$ & 1 & 3. ००E - ๑7 & $1.80 E-03$ \\
\hline$U-238$ & $M$ & 1 & $3.00 E-07$ & $1.80 E-03$ \\
\hline Th - 234 & $\mathrm{~S}$ & 1 & $3.0 \odot E-\odot 7$ & 1. 80E-०3 \\
\hline
\end{tabular}


Appendix A

$\mathrm{Pa}-234 \mathrm{~m}$
$\mathrm{~Pa}-234$
$\mathrm{Al}-26$
$\mathrm{Be}-7$
$\mathrm{Cm}-243$
$\mathrm{Am}-243$
$\mathrm{~Np}-239$
$\mathrm{Pu}-239$
$\mathrm{U}-235$
$\mathrm{Th}-231$
$\mathrm{~Pa}-231$
$\mathrm{AC}-227$
$\mathrm{Th}-227$
$\mathrm{Ra}-223$
$\mathrm{Rn}-219$
$\mathrm{Po}-215$
$\mathrm{~Pb}-211$
$\mathrm{Bi}-211$
$\mathrm{Tl}-207$
$\mathrm{Po}-211$
$\mathrm{Fr}-223$
$\mathrm{Co}-58$
$\mathrm{Co}-60$
$\mathrm{CS}-134$
$\mathrm{Eu}-152$
$\mathrm{Gd}-152$
$\mathrm{Eu}-154$
$\mathrm{Eu}-155$
$\mathrm{Nb}-94$
$\mathrm{Sb}-125$
$\mathrm{Te}-125 \mathrm{~m}$
$\mathrm{H}-3$

$\begin{array}{ll}M & 1 \\ M & 1 \\ M & 1 \\ M & 1 \\ M & 1 \\ M & 1 \\ M & 1 \\ M & 1 \\ M & 1 \\ S & 1 \\ M & 1 \\ M & 1 \\ S & 1 \\ M & 1 \\ G & 0 \\ M & 1 \\ M & 1 \\ M & 1 \\ M & 1 \\ M & 1 \\ M & 1 \\ M & 1 \\ M & 1 \\ F & 1 \\ M & 1 \\ M & 1 \\ M & 1 \\ M & 1 \\ M & 1 \\ M & 1 \\ M & 0 \\ V & \end{array}$

\begin{tabular}{|c|c|}
\hline 3. $00 \mathrm{E}-07$ & $1.80 \mathrm{E}-03$ \\
\hline 3. $\odot \odot E-\odot 7$ & $1.80 \mathrm{E}-03$ \\
\hline 3. ००E - ๑7 & $1.80 \mathrm{E}-03$ \\
\hline 3. $\odot \odot E-\odot 7$ & $1.80 E-\odot 3$ \\
\hline 3. $\odot \odot E-\odot 7$ & $1.80 \mathrm{E}-03$ \\
\hline 3. $0 \odot \mathrm{E}-\odot 7$ & $1.80 \mathrm{E}-03$ \\
\hline $3 . \odot \odot E-\odot 7$ & $1.80 \mathrm{E}-03$ \\
\hline 3. ๑९E - ๑7 & $1.80 E-\odot 3$ \\
\hline 3. ००E- - 7 & $1.80 \mathrm{E}-03$ \\
\hline 3. $\odot \odot E-\odot 7$ & $1.80 \mathrm{E}-03$ \\
\hline 3. $\odot \odot E-\odot 7$ & $1.80 \mathrm{E}-03$ \\
\hline 3. $\odot \odot E-\odot 7$ & $1.80 \mathrm{E}-03$ \\
\hline 3. ००E - ๑7 & $1.80 \mathrm{E}-03$ \\
\hline $3 . \odot \odot E-\odot 7$ & $1.80 \mathrm{E}-\odot 3$ \\
\hline$\odot . \odot \odot E+\odot \odot$ & $\odot . \odot \odot E+\odot \odot$ \\
\hline $3.00 \mathrm{E}-07$ & $1.80 E-03$ \\
\hline $3.00 \mathrm{E}-\odot 7$ & 1. $80 \mathrm{E}-03$ \\
\hline 3. ००E-๑7 & $1.80 \mathrm{E}-03$ \\
\hline 3. $\odot \odot E-\odot 7$ & $1.80 \mathrm{E}-03$ \\
\hline 3. ००E - ๑7 & $1.80 \mathrm{E}-03$ \\
\hline 3. ๑९E - ๑7 & $1.80 E-03$ \\
\hline 3. $\odot \odot E-\odot 7$ & $1.80 \mathrm{E}-03$ \\
\hline 3. ००E-๑7 & $1.80 \mathrm{E}-03$ \\
\hline 3. $\odot \odot E-\odot 7$ & $1.80 \mathrm{E}-03$ \\
\hline $3.00 E-\odot 7$ & 1. $80 \mathrm{E}-03$ \\
\hline 3. $0 \odot \mathrm{E}-\odot 7$ & $1.80 \mathrm{E}-03$ \\
\hline 3. ००E-๑7 & $1.80 \mathrm{E}-\odot 3$ \\
\hline 3. $\odot \odot E-\odot 7$ & $1.80 \mathrm{E}-03$ \\
\hline 3. $0 \odot \mathrm{E}-\odot 7$ & $1.80 \mathrm{E}-03$ \\
\hline $3 . \odot \odot E-\odot 7$ & $1.80 \mathrm{E}-03$ \\
\hline 3. $\odot \odot E-\odot 7$ & 1. $80 \mathrm{E}-\odot 3$ \\
\hline$\odot . \odot \odot E+\odot \odot$ & $\odot . \odot \odot E+\odot \odot$ \\
\hline
\end{tabular}


VALUES FOR RADIONUCLIDE-DEPENDENT PARAMETERS

\begin{tabular}{|c|c|c|c|c|c|}
\hline \multirow[b]{2}{*}{ Nuclide } & \multicolumn{3}{|c|}{ DECAY CONSTANT (PER DAY) } & \multicolumn{2}{|c|}{ TRANSFER COEFFICIENT } \\
\hline & $\begin{array}{l}\text { Radio- } \\
\text { active (1) }\end{array}$ & Surface & Water & Milk (2) & Meat (3) \\
\hline Cs -137 & $6.32 \mathrm{E}-05$ & $5.48 E-05$ & $\odot . \odot \odot E+\odot \odot$ & $1.0 \odot E-02$ & $5.00 \mathrm{E}-02$ \\
\hline $\mathrm{Ba}-137 \mathrm{~m}$ & $3.91 \mathrm{E}+02$ & $5.48 E-\odot 5$ & $\odot . \odot \odot E+\odot \odot$ & $5 . \odot \odot E-\odot 4$ & $2 . \odot \odot E-\odot 4$ \\
\hline Am-241 & $4.39 E-06$ & $5.48 E-\odot 5$ & $\odot . \odot \odot E+\odot \odot$ & $2 . \odot \odot E-\odot 6$ & $5.00 E-\odot 5$ \\
\hline $\mathrm{Np}-237$ & $8.87 \mathrm{E}-10$ & $5.48 \mathrm{E}-\odot 5$ & $\odot . \odot \odot E+\odot \odot$ & $1.00 \mathrm{E}-\odot 5$ & $1.00 \mathrm{E}-03$ \\
\hline$P a-233$ & $2.57 \mathrm{E}-02$ & $5.48 E-\odot 5$ & $\odot . \odot \odot E+\odot \odot$ & $5 . \odot \odot E-\odot 6$ & $5.0 \odot E-\odot 6$ \\
\hline$U-233$ & $1.20 E-\odot 8$ & $5.48 \mathrm{E}-05$ & $\odot . \odot \odot E+\odot \odot$ & $4.0 \odot E-\odot 4$ & $8.00 E-\odot 4$ \\
\hline Th-229 & $2.58 \mathrm{E}-07$ & $5.48 \mathrm{E}-05$ & $\odot . \odot \odot E+\odot \odot$ & $5.0 \odot E-\odot 6$ & $1.00 E-04$ \\
\hline $\mathrm{Ra}-225$ & $4.68 \mathrm{E}-02$ & $5.48 E-05$ & $\odot . \odot \odot E+\odot \odot$ & $1.0 \odot E-\odot 3$ & $2.00 E-\odot 3$ \\
\hline Ac -225 & $6.93 E-02$ & $5.48 E-05$ & $\odot . \odot \odot E+\odot \odot$ & $2 . \odot \odot E-\odot 6$ & $2.00 E-\odot 5$ \\
\hline $\mathrm{Fr}-221$ & $2.08 \mathrm{E}+02$ & $5.48 \mathrm{E}-05$ & $\odot . \odot \odot E+\odot \odot$ & $8.00 E-03$ & $3.00 \mathrm{E}-02$ \\
\hline At -217 & $1.85 E+\odot 6$ & $5.48 \mathrm{E}-05$ & $\odot . \odot \odot E+\odot \odot$ & $1 . \odot \odot E-\odot 2$ & 1. $\odot \odot E-\odot 2$ \\
\hline $\mathrm{Bi}-213$ & $2.19 \mathrm{E}+01$ & $5.48 \mathrm{E}-05$ & $\odot . \odot \odot E+\odot \odot$ & $1.00 \mathrm{E}-03$ & $2.00 E-\odot 3$ \\
\hline Po- 213 & $1.43 \mathrm{E}+10$ & $5.48 \mathrm{E}-05$ & $\odot . \odot \odot E+\odot \odot$ & $4.00 E-\odot 4$ & $5.00 E-03$ \\
\hline$P b-209$ & $5.11 \mathrm{E}+\odot \odot$ & $5.48 \mathrm{E}-05$ & $\odot . \odot \odot E+\odot \odot$ & $3 . \odot \odot E-\odot 4$ & $8.00 \mathrm{E}-\odot 4$ \\
\hline Tl-209 & $4.54 \mathrm{E}+02$ & $5.48 E-05$ & $\odot . \odot \odot E+\odot \odot$ & $3.00 \mathrm{E}-\odot 3$ & $2.0 \odot E-\odot 2$ \\
\hline $\mathrm{Pu}-238$ & $2.16 \mathrm{E}-05$ & $5.48 E-\odot 5$ & $\odot . \odot \odot E+\odot \odot$ & $1 . \odot \odot E-\odot 6$ & $1.0 \odot E-\odot 4$ \\
\hline$U-234$ & $7.76 \mathrm{E}-\odot 9$ & $5.48 E-05$ & $\odot . \odot \odot E+\odot \odot$ & $4.0 \odot E-\odot 4$ & $8.0 \odot E-\odot 4$ \\
\hline Th - 230 & $2.46 E-\odot 8$ & $5.48 \mathrm{E}-05$ & $\odot . \odot \odot E+\odot \odot$ & $5.0 \odot E-\odot 6$ & $1.00 E-\odot 4$ \\
\hline $\mathrm{Ra}-226$ & $1.19 \mathrm{E}-06$ & $5.48 E-\odot 5$ & $\odot . \odot \odot E+\odot \odot$ & 1. ๑๑E - ๑3 & 2. $\odot \odot E-\odot 3$ \\
\hline$R n-222$ & $1.81 \mathrm{E}-01$ & $5.48 E-\odot 5$ & $\odot . \odot \odot E+\odot \odot$ & $\odot . \odot \odot E+\odot \odot$ & $\odot . \odot \odot E+\odot \odot$ \\
\hline Po- 218 & $3.27 \mathrm{E}+\Theta 2$ & $5.48 E-05$ & $\odot . \odot \odot E+\odot \odot$ & $4.0 \odot E-\odot 4$ & $5.00 \mathrm{E}-03$ \\
\hline $\mathrm{Pb}-214$ & $3.72 \mathrm{E}+01$ & $5.48 E-05$ & $\odot . \odot \odot E+\odot \odot$ & $3 . \odot \odot E-\odot 4$ & 8. $\odot \odot E-\odot 4$ \\
\hline $\mathrm{Bi}-214$ & $5.02 \mathrm{E}+01$ & $5.48 E-05$ & $\odot . \odot \odot E+\odot \odot$ & $1.00 \mathrm{E}-\odot 3$ & $2.00 \mathrm{E}-\odot 3$ \\
\hline Po- 214 & $3.64 \mathrm{E}+08$ & $5.48 \mathrm{E}-05$ & $\odot . \odot \odot E+\odot \odot$ & $4.0 \odot E-\odot 4$ & $5.00 E-03$ \\
\hline $\mathrm{Pb}-210$ & $8.51 E-05$ & $5.48 E-\odot 5$ & $\odot . \odot \odot E+\odot \odot$ & $3 . \odot \odot E-\odot 4$ & 8. $\odot \odot E-\odot 4$ \\
\hline $\mathrm{Bi}-210$ & $1.38 \mathrm{E}-01$ & $5.48 E-05$ & $\odot . \odot \odot E+\odot \odot$ & $1.0 \odot E-\odot 3$ & $2 . \odot \odot E-\odot 3$ \\
\hline Po -210 & $5.01 E-03$ & $5.48 E-05$ & $\odot . \odot \odot E+\odot \odot$ & $4.0 \odot E-\odot 4$ & $5.00 \mathrm{E}-03$ \\
\hline At -218 & $2.99 \mathrm{E}+\odot 4$ & $5.48 E-\odot 5$ & $\odot . \odot \odot E+\odot \odot$ & 1. $\odot \odot E-\odot 2$ & 1. $\odot \odot E-\odot 2$ \\
\hline Sr - 9๑ & $6.52 \mathrm{E}-05$ & $5.48 \mathrm{E}-05$ & $\odot . \odot \odot E+\odot \odot$ & $2.0 \odot E-\odot 3$ & $1.00 \mathrm{E}-02$ \\
\hline$Y-90$ & $2.60 \mathrm{E}-01$ & $5.48 E-05$ & $\odot . \odot \odot E+\odot \odot$ & $6.00 \mathrm{E}-05$ & $2.00 E-03$ \\
\hline $\mathrm{K}-40$ & $1.48 \mathrm{E}-12$ & $5.48 E-\odot 5$ & $\odot . \odot \odot E+\odot \odot$ & $7 . \odot \odot E-\odot 3$ & $2 . \odot \odot E-\odot 2$ \\
\hline Th- 232 & $1.35 E-13$ & $5.48 E-05$ & $\odot . \odot \odot E+\odot \odot$ & $5.00 \mathrm{E}-06$ & $1.00 E-04$ \\
\hline $\mathrm{Ra}-228$ & $3.30 E-04$ & $5.48 E-05$ & $\odot . \odot \odot E+\odot \odot$ & 1. $\odot \odot E-\odot 3$ & 2. $\odot \odot E-\odot 3$ \\
\hline Ac -228 & $2.71 \mathrm{E}+\odot \odot$ & $5.48 \mathrm{E}-05$ & $\odot . \odot \odot E+\odot \odot$ & $2 . \odot \odot E-\odot 6$ & $2.0 \odot E-\odot 5$ \\
\hline Th - 228 & $9.92 \mathrm{E}-\odot 4$ & $5.48 \mathrm{E}-05$ & $\odot . \odot \odot E+\odot \odot$ & $5.00 \mathrm{E}-06$ & $1.00 E-04$ \\
\hline $\mathrm{Ra}-224$ & $1.89 \mathrm{E}-01$ & $5.48 E-05$ & $\odot . \odot \odot E+\odot \odot$ & 1. $\odot \odot E-\odot 3$ & 2. $\odot \odot E-\odot 3$ \\
\hline$R n-220$ & $1 . \odot 8 \mathrm{E}+03$ & $5.48 E-05$ & $\odot . \odot \odot E+\odot \odot$ & $\odot . \odot \odot E+\odot \odot$ & $\odot . \odot \odot E+\odot \odot$ \\
\hline Po- 216 & $3.99 E+05$ & $5.48 E-05$ & $\odot . \odot \odot E+\odot \odot$ & $4.0 \odot E-\odot 4$ & $5.00 \mathrm{E}-03$ \\
\hline $\mathrm{Pb}-212$ & $1.56 \mathrm{E}+\odot \odot$ & $5.48 E-05$ & $\odot . \odot \odot E+\odot \odot$ & $3 . \odot \odot E-\odot 4$ & $8.0 \odot E-\odot 4$ \\
\hline $\mathrm{Bi}-212$ & $1.65 \mathrm{E}+01$ & $5.48 E-05$ & $\odot . \odot \odot E+\odot \odot$ & $1.00 \mathrm{E}-03$ & $2.00 E-03$ \\
\hline Po-212 & $1.96 \mathrm{E}+11$ & $5.48 \mathrm{E}-05$ & $\odot . \odot \odot E+\odot \odot$ & $4.0 \odot E-\odot 4$ & $5.00 E-\odot 3$ \\
\hline Tl-208 & $3.25 \mathrm{E}+02$ & $5.48 E-\odot 5$ & $\odot . \odot \odot E+\odot \odot$ & $3 . \odot \odot E-\odot 3$ & $2 . \odot \odot E-\odot 2$ \\
\hline$U-238$ & $4.25 E-13$ & $5.48 \mathrm{E}-\odot 5$ & $\odot . \odot \odot E+\odot \odot$ & $4.0 \odot E-\odot 4$ & $8.0 \odot E-\odot 4$ \\
\hline
\end{tabular}


Appendix A

\begin{tabular}{|c|c|c|c|c|c|}
\hline Th-234 & $2.88 E-02$ & $5.48 E-05$ & $\odot . \odot \odot E+\odot \odot$ & $5 . \odot \odot E-\odot 6$ & 1. ००E-०4 \\
\hline $\mathrm{Pa}-234 \mathrm{~m}$ & $8.53 E+02$ & $5.48 E-05$ & $\odot . \odot \odot E+\odot \odot$ & $5.00 \mathrm{E}-\odot 6$ & $5.00 \mathrm{E}-06$ \\
\hline $\mathrm{Pa}-234$ & $2.48 E+\odot \odot$ & $5.48 E-05$ & $\odot . \odot \odot E+\odot \odot$ & $5 . \odot \odot E-\odot 6$ & 5. $00 \mathrm{E}-\odot 6$ \\
\hline Al-26 & $2.65 E-\odot 9$ & $5.48 E-05$ & $\odot . \odot \odot E+\odot \odot$ & 2. ๑०E-๑4 & $5.00 \mathrm{E}-04$ \\
\hline $\mathrm{Be}-7$ & 1. 3०E- $\odot 2$ & $5.48 E-05$ & $\odot . \odot \odot E+\odot \odot$ & $2 . \odot \odot E-\odot 6$ & 5. ००E-๑3 \\
\hline $\mathrm{Cm}-243$ & $6.66 E-05$ & $5.48 E-05$ & $\odot . \odot \odot E+\odot \odot$ & 2. $\odot \odot E-\odot 6$ & 2. $00 \mathrm{E}-05$ \\
\hline Am- 243 & $2.57 E-07$ & $5.48 E-05$ & $\odot . \odot \odot E+\odot \odot$ & 2. ๑०E - ०6 & $5.00 \mathrm{E}-\odot 5$ \\
\hline Np - 239 & 2. $94 \mathrm{E}-01$ & $5.48 E-05$ & $\odot . \odot \odot E+\odot \odot$ & 1. ००E- $\odot 5$ & 1. $\odot \odot E-\odot 3$ \\
\hline Pu-239 & $7.88 E-08$ & $5.48 E-05$ & $\odot . \odot \odot E+\odot \odot$ & 1. ००Е-๑6 & 1. $\odot \odot E-\odot 4$ \\
\hline$U-235$ & 2. $70 \mathrm{E}-12$ & $5.48 E-05$ & $\odot . \odot \odot E+\odot \odot$ & 4. ००Е-๑4 & 8. $.0 \mathrm{E}-\odot 4$ \\
\hline Th-231 & $6.52 E-01$ & $5.48 E-05$ & $\odot . \odot \odot E+\odot \odot$ & 5. ๑०Е - ०6 & 1. $\odot \odot E-\odot 4$ \\
\hline$P a-231$ & $5.79 E-08$ & $5.48 E-05$ & $\odot . \odot \odot E+\odot \odot$ & 5. ००E - ०6 & $5.00 \mathrm{E}-06$ \\
\hline$A c-227$ & $8.71 E-05$ & $5.48 E-05$ & $\odot . \odot \odot E+\odot \odot$ & 2. $\odot ० E-\odot 6$ & 2. $00 \mathrm{E}-\odot 5$ \\
\hline Th- 227 & $3.70 E-\odot 2$ & $5.48 E-05$ & $\odot . \odot \odot E+\odot \odot$ & $5 . \odot \odot E-\odot 6$ & 1. $.0 \mathrm{E}-\odot 4$ \\
\hline $\mathrm{Ra}-223$ & $6.06 E-02$ & $5.48 E-05$ & $\odot . \odot \odot E+\odot \odot$ & 1. $00 \mathrm{E}-03$ & 2. .०० - ०3 \\
\hline Rn- 219 & $1.51 E+04$ & $5.48 E-05$ & $\odot . \odot \odot E+\odot \odot$ & $\odot . \odot \odot E+\odot \odot$ & $\odot . \odot \odot E+\odot \odot$ \\
\hline Po- 215 & $3.36 E+07$ & $5.48 E-05$ & $\odot . \odot \odot E+\odot \odot$ & $4 . \odot \odot E-\odot 4$ & 5. ००E- - \\
\hline $\mathrm{Pb}-211$ & $2.76 E+01$ & $5.48 E-05$ & $\odot . \odot \odot E+\odot \odot$ & 3. $00 \mathrm{E}-04$ & 8. $00 \mathrm{E}-04$ \\
\hline Bi-211 & $4.66 E+02$ & $5.48 E-05$ & $\odot . \odot \odot E+\odot \odot$ & 1. $00 \mathrm{E}-03$ & 2. .०० - ०3 \\
\hline Tl - 207 & 2. $09 \mathrm{E}+02$ & $5.48 E-05$ & $\odot . \odot \odot E+\odot \odot$ & 3. ००E - ०3 & 2. $\odot \odot E-\odot 2$ \\
\hline Po- 211 & $1.16 \mathrm{E}+05$ & $5.48 E-05$ & $\odot . \odot \odot E+\odot \odot$ & $4.0 \odot E-\odot 4$ & 5. ००E-०3 \\
\hline $\mathrm{Fr}-223$ & $4.58 E+01$ & $5.48 E-05$ & $\odot . \odot \odot E+\odot \odot$ & 8. ००E-०3 & 3. ००E-०2 \\
\hline Co-58 & $9.79 E-03$ & $5.48 E-05$ & $\odot . \odot \odot E+\odot \odot$ & 2. ००E - ०3 & 3. $0 \odot \mathrm{E}-02$ \\
\hline Co- 60 & $3.60 E-\odot 4$ & $5.48 E-05$ & $\odot . \odot \odot E+\odot \odot$ & $2 . \odot \odot E-\odot 3$ & 3. ००E-๑2 \\
\hline Cs - 134 & $9.20 E-04$ & $5.48 E-05$ & $\odot . \odot \odot E+\odot \odot$ & 1. $00 \mathrm{E}-02$ & 5. $00 \mathrm{E}-02$ \\
\hline Eu-152 & $1.32 \mathrm{E}+00$ & $5.48 E-05$ & $\odot . \odot \odot E+\odot \odot$ & $6.00 \mathrm{E}-\odot 5$ & $2.0 \odot E-\odot 3$ \\
\hline Gd-152 & $1.76 \mathrm{E}-17$ & $5.48 E-05$ & $\odot . \odot \odot E+\odot \odot$ & $6.00 \mathrm{E}-05$ & 2. ००E-०3 \\
\hline Eu-154 & $1.78 \mathrm{E}+\odot \odot$ & $5.48 E-05$ & $\odot . \odot \odot E+\odot \odot$ & $6.0 \odot E-\odot 5$ & $2 . \odot \odot E-\odot 3$ \\
\hline Eu-155 & $2.16 E-04$ & $5.48 E-05$ & $\odot . \odot \odot E+\odot \odot$ & $6.00 \mathrm{E}-05$ & 2. .०० - ०3 \\
\hline Nb - 94 & $9.35 E-\odot 8$ & $5.48 E-05$ & $\odot . \odot \odot E+\odot \odot$ & $2 . \odot \odot E-\odot 6$ & 3. ००E-๑7 \\
\hline Sb-125 & $6.85 E-04$ & $5.48 E-05$ & $\odot . \odot \odot E+\odot \odot$ & 1. ००E-०4 & 1. ००E-०3 \\
\hline Te-125m & 1. $20 \mathrm{E}-\odot 2$ & $5.48 E-05$ & $\odot . \odot \odot E+\odot \odot$ & $5 . \odot \odot E-\odot 4$ & 7. ००E-๑3 \\
\hline $\mathrm{H}-3$ & $1.54 \mathrm{E}-04$ & $5.48 E-05$ & $\odot . \odot \odot E+\odot \odot$ & $\odot . \odot \odot E+\odot \odot$ & $\odot . \odot \odot E+\odot \odot$ \\
\hline
\end{tabular}

FOOTNOTES :

(1) Fraction of animal's daily intake of nuclide which appears in each $L$ of milk (days/L)

(2) Fraction of animal's daily intake of nuclide which appears in each $\mathrm{kg}$ of meat (days $/ \mathrm{kg}$ ) 
VALUES FOR RADIONUCLIDE-DEPENDENT PARAMETERS

\begin{tabular}{|c|c|c|c|c|}
\hline \multirow[b]{2}{*}{ Nuclide } & \multicolumn{2}{|c|}{$\begin{array}{l}\text { CONCENTRATION } \\
\text { UPTAKE FACTOR }\end{array}$} & \multicolumn{2}{|c|}{ GI UPTAKE FRACTION } \\
\hline & Forage (1) & Edible (2) & Inhalation & Ingestion \\
\hline Cs-137 & 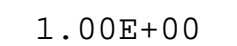 & 2. .०E - ०1 & 1. ००E+०० & 1. ००E+०० \\
\hline $\mathrm{Ba}-137 \mathrm{~m}$ & 1. ००E- 01 & 1. ००E - ०2 & 2. $.0 \mathrm{E}-\odot 1$ & 2. ००E - ०1 \\
\hline Am-241 & 1. ००E- 01 & 1. ००E-०3 & 5. ००E - ०4 & $5.0 \odot E-\odot 4$ \\
\hline$N p-237$ & 1. ००Е- 01 & $2.00 \mathrm{E}-\odot 2$ & 5. ००E- - 4 & 5. ००Е- - \\
\hline $\mathrm{Pa}-233$ & 1. ००E- 01 & 1. ००E- 02 & $5.0 \odot E-\odot 4$ & 5. ००E - ๑4 \\
\hline$U-233$ & 1. ००E- 01 & 2. ००E-०3 & $2.0 \odot E-\odot 2$ & 2. ००E-๑2 \\
\hline Th - 229 & 1. ००E- 01 & 1. ००E - ०3 & 5. ००E- - 4 & $5.0 \odot E-04$ \\
\hline $\mathrm{Ra}-225$ & 2. ००E- 01 & $4.00 \mathrm{E}-\odot 2$ & 2. $00 \mathrm{E}-01$ & 2. ००E- 01 \\
\hline$A c-225$ & 1. ๑७Е- ०1 & 1. ००E-०3 & 5. ००Е - ๑4 & 5. ๑७Е-๑4 \\
\hline Fr- 221 & 1. ०७Е- $० 1$ & 3. ००E-०2 & 1. ००E+०० & 1. ००E+०० \\
\hline At -217 & $9.0 \odot E-01$ & 2. . ०० - 01 & 1. ००E+०० & 1. ००E+०० \\
\hline $\mathrm{Bi}-213$ & 5. ००E- 01 & 1. $00 \mathrm{E}-01$ & 5. ००E- - 2 & 5. ००E- - \\
\hline Po- 213 & 1. ๑७Е- ०1 & 1. ००E-०3 & 1. ००E - ०1 & 1. ๑๑Е - ०1 \\
\hline$P b-209$ & 1. ०७Е- ०1 & 4. ००E-०3 & 2. $0 \odot E-\odot 1$ & 2. ๑๑Е - ๑1 \\
\hline Tl- 209 & $6.00 \mathrm{E}-01$ & 2. .०० - ०1 & 1. $\odot \odot E+\odot \odot$ & 1. ००E+०० \\
\hline Pu- 238 & 1. ๑७Е- -1 & 1. ००E-०3 & 5. ००E - ०4 & 5. ๑७Е - ๑४ \\
\hline U-234 & 1. ๑७Е- -1 & 2. ००E-०3 & 2. ००E - ०2 & 2. ๑७E - ๑2 \\
\hline Th - 230 & 1. ०७Е-०1 & 1. ००E-०3 & 5. ००E - ०4 & 5. ๑७Е - ๑4 \\
\hline $\mathrm{Ra}-226$ & 2. ๑७E - ๑1 & 4. ००E - ๑2 & 2. ००E- -1 & 2. ๑๑E - ०1 \\
\hline$R n-222$ & ๑ . ०७Е+०० & $\odot . \odot \odot E+\odot \odot$ & $\odot . \odot \odot E+\odot \odot$ & $\odot . \odot \odot E+\odot \odot$ \\
\hline Po- 218 & 1. ०७Е- $९ 1$ & 1. ००E-०3 & 1. ००E - ०1 & 1. ๑๑Е - ०1 \\
\hline $\mathrm{Pb}-214$ & 1. ००Е-०1 & 4. ००E- - & 2. $. \odot E-\odot 1$ & 2. ๑०Е- $\odot 1$ \\
\hline Bi-214 & 5. ००E- 01 & 1. .0०E- - 1 & $5.0 \odot E-\odot 2$ & 5. ००E- $\odot 2$ \\
\hline Po- 214 & 1. ००E- 01 & 1. ००E- - & $1.00 \mathrm{E}-01$ & 1. ००E- 01 \\
\hline $\mathrm{Pb}-210$ & 1. ००E- 01 & 4. ००E- - & 2. $00 \mathrm{E}-01$ & 2. ००E- 01 \\
\hline $\mathrm{Bi}-210$ & 5. ००Е- $\odot 1$ & 1. $00 \mathrm{E}-01$ & 5. ००E-๑2 & 5. ๑०Е- - \\
\hline Po- 210 & 1. ००E- 01 & 1. ००E- - & 1. ००E-०1 & 1. ००E- 01 \\
\hline At - 218 & $9.00 \mathrm{-}-01$ & 2. $00 \mathrm{E}-01$ & 1. ००E+०० & 1. ००E+०० \\
\hline Sr-90 & 4. .०E+०० & 3. 00E- - 1 & 3. ००E- - & 3. ००E- 01 \\
\hline$Y-90$ & 1. ००E-०1 & 2. $0 \odot E-\odot 3$ & 1. ००E-๑4 & 1. ००Е-०4 \\
\hline$K-40$ & 3. ००E+०० & 3. 0०E - ०1 & 1. $\odot \odot E+\odot \odot$ & 1. ००E+०० \\
\hline Th-232 & 1. ००E- 01 & 1. ००E- - & $5.00 E-\odot 4$ & 5. ००E- - \\
\hline $\mathrm{Ra}-228$ & 2. .०० - 01 & $4.00 E-\odot 2$ & 2. $00 \mathrm{E}-01$ & 2. ००E- 01 \\
\hline$A c-228$ & 1. ००E- 01 & 1. ००E-०3 & 5. ००E - ๑4 & 5. ००Е- - \\
\hline Th - 228 & 1. ००E- 01 & 1. ००E-०3 & $5.00 E-\odot 4$ & 5. ००Е- - \\
\hline $\mathrm{Ra}-224$ & 2. .०E- 01 & 4. ००E- ०2 & 2. $00 \mathrm{E}-01$ & 2. ००E- 01 \\
\hline$R n-220$ & ๑ . ००Е+०० & $\odot . \odot \odot E+\odot \odot$ & $\odot . \odot \odot E+\odot \odot$ & $\odot . \odot \odot E+\odot \odot$ \\
\hline Po- 216 & 1. ००E- 01 & 1. ००E-०3 & 1. $00 \mathrm{E}-01$ & 1. ००E- 01 \\
\hline $\mathrm{Pb}-212$ & 1. ००Е- 01 & $4.00 E-\odot 3$ & 2. $0 \odot E-\odot 1$ & 2. ००E - 01 \\
\hline $\mathrm{Bi}-212$ & 5. ००Е- 01 & 1. $00 \mathrm{E}-01$ & 5. ००E - ๑2 & 5. ००E- - \\
\hline Po- 212 & 1. ००E- 01 & 1. ००E-०3 & 1. $00 \mathrm{E}-01$ & 1. .०Е- 01 \\
\hline Tl- 208 & 6. .०Е- 01 & 2. .०० - ०1 & 1. $\odot \odot E+\odot \odot$ & 1. ००E+०० \\
\hline U-238 & 1. ๑७Е-०1 & 2. ००E-๑3 & 2. ००E-๑2 & 2. ๑७Е-๑2 \\
\hline
\end{tabular}




\begin{tabular}{|c|c|c|c|c|}
\hline Th-234 & 1. ००E - 01 & 1. ००E- - & 5. ००E - ०4 & $5.0 \odot E-\odot 4$ \\
\hline$P a-234 m$ & 1. . ००E-०1 & 1. ००E-०2 & 5. $00 \mathrm{E}-\odot 4$ & $5 . \odot \odot E-\odot 4$ \\
\hline $\mathrm{Pa}-234$ & 1. ००E- ०1 & 1. $00 \mathrm{E}-02$ & $5.00 E-\odot 4$ & $5.00 E-\odot 4$ \\
\hline Al- 26 & 1. ๑७E- $९ 1$ & 4. ००E-๑3 & 1. ००E - ०2 & 1. $\odot \odot E-\odot 2$ \\
\hline $\mathrm{Be}-7$ & 1. ๑๑Е-๑1 & $4 . \odot \odot E-\odot 3$ & 5. ๑७Е - ๑3 & 5. ००Е-๑3 \\
\hline $\mathrm{Cm}-243$ & 1. ००E-०1 & 1. .०० - ०3 & $5.00 E-\odot 4$ & $5.00 E-04$ \\
\hline Am- 243 & 1. ००E- ०1 & 1. .0०E-०3 & $5.00 E-\odot 4$ & $5.0 \odot E-\odot 4$ \\
\hline$N p-239$ & 1. ๑०Е- ०1 & 2. ००E-๑2 & 5. ๑०E - ๑4 & 5. ००E-๑4 \\
\hline Pu- 239 & 1. ००E-०1 & 1. .०० - ०3 & $5.00 E-\odot 4$ & $5.0 \odot E-\odot 4$ \\
\hline$U-235$ & 1. ๑०Е-०1 & 2. .०Е-०3 & $2 . \odot \odot E-\odot 2$ & 2. $\odot \odot E-\odot 2$ \\
\hline Th-231 & 1. ००E-०1 & 1. .०० - ०3 & $5.00 E-\odot 4$ & $5.00 E-\odot 4$ \\
\hline $\mathrm{Pa}-231$ & 1. .०० - 01 & 1. ००E-०2 & 5. ००E - 04 & $5.00 E-04$ \\
\hline$A C-227$ & 1. .०० - 01 & 1. ००E-๑3 & 5. ००E - ๑4 & 5. ๑७E- - ४4 \\
\hline Th-227 & 1. .०० - 01 & 1. ००E-๑3 & 5. ००E - ๑4 & 5. ๑७E - ๑4 \\
\hline $\mathrm{Ra}-223$ & 2. ००E-०1 & 4. .०० - ०2 & 2. .०E - ०1 & 2. . $00 \mathrm{E}-01$ \\
\hline Rn- 219 & $\odot . \odot \odot E+\odot \odot$ & $\odot . \odot \odot E+\odot \odot$ & $\odot . \odot \odot E+\odot \odot$ & $\odot . \odot \odot E+\odot \odot$ \\
\hline Po- 215 & 1. ๑๑Е- ०1 & 1. $.0 \mathrm{E}-\odot 3$ & 1. $\odot \odot E-\odot 1$ & 1. $. \odot E-\odot 1$ \\
\hline $\mathrm{Pb}-211$ & 1. ००E-०1 & 4. .०Е- - ०3 & 2. $.0 \mathrm{E}-01$ & 2. . $00 \mathrm{E}-01$ \\
\hline Bi-211 & 5. ००Е- 01 & 1. $00 \mathrm{E}-01$ & $5.00 \mathrm{E}-02$ & $5.0 \odot E-02$ \\
\hline Tl- 207 & 6. ००Е- 01 & 2. $00 \mathrm{E}-01$ & 1. $.0 \mathrm{E}+0 \odot$ & 1. $.0 \mathrm{E}+0 \odot$ \\
\hline Po- 211 & 1. ००Е-०1 & 1. .०० - ०3 & 1. $00 \mathrm{E}-01$ & 1. $00 \mathrm{E}-01$ \\
\hline Fr - 223 & 1. ००E-०1 & 3. $00 \mathrm{E}-02$ & 1. $\odot ० E+\odot \odot$ & 1. $.0 \mathrm{E}+00$ \\
\hline $\mathrm{Co}-58$ & 2. $\odot \odot E+\odot \odot$ & 8. $00 \mathrm{E}-02$ & 1. $00 \mathrm{E}-01$ & 1. $00 \mathrm{E}-01$ \\
\hline $\mathrm{Co}-60$ & $2 . \odot \odot E+\odot \odot$ & 8. ००E-๑2 & 1. $\odot \odot E-\odot 1$ & 1. $\odot \odot E-\odot 1$ \\
\hline Cs - 134 & 1. $\odot \odot E+\odot \odot$ & 2. $00 \mathrm{E}-01$ & 1. $.0 \mathrm{E}+0 \odot$ & 1. $00 \mathrm{E}+0 \odot$ \\
\hline Eu-152 & 1. $\odot \odot E-\odot 1$ & $2.0 \odot E-\odot 3$ & $5.00 \mathrm{E}-\odot 4$ & $5.0 \odot E-\odot 4$ \\
\hline Gd-152 & 1. ००E-०1 & 2. ००E-०3 & $5 . \odot \odot E-\odot 4$ & $5.0 \odot E-\odot 4$ \\
\hline Eu-154 & 1. ๑०Е-๑1 & $2 . \odot \odot E-\odot 3$ & 5. ๑०E - ๑4 & $5 . \odot \odot E-\odot 4$ \\
\hline Eu-155 & 1. $0 \odot E-01$ & 2. ००E-०3 & $5.00 \mathrm{E}-04$ & $5.00 \mathrm{E}-04$ \\
\hline $\mathrm{Nb}-94$ & 1. ๑०Е-०1 & 1. $. \odot E-\odot 2$ & 1. $\odot \odot E-\odot 2$ & 1. $\odot \odot E-\odot 2$ \\
\hline Sb-125 & 1. $.0 \mathrm{E}-01$ & 1. ००E- 02 & 1. $.0 \mathrm{E}-01$ & 1. .०Е- - 1 \\
\hline Te-125m & $1.30 E+\odot \odot$ & 1. $. \odot E-\odot 1$ & 3. ๑०E - ०1 & 3. ๑०E - ०1 \\
\hline $\mathrm{H}-3$ & $\odot . \odot \odot E+\odot \odot$ & $\odot . \odot \odot E+\odot \odot$ & 1. $\odot \odot E+\odot \odot$ & 1. $\odot \odot E+\odot \odot$ \\
\hline
\end{tabular}

FOOTNOTES: (1) Concentration factor for uptake of nuclide from soil for pasture and forage (in $\mathrm{pCi} / \mathrm{kg}$ dry weight per $\mathrm{pCi} / \mathrm{kg}$ dry soil)

(2) Concentration factor for uptake of nuclide from soil by edible parts of crops (in $\mathrm{pCi} / \mathrm{kg}$ wet weight per $\mathrm{pCi} / \mathrm{kg}$ dry soil) 
oct 26, 2006 08:38 am

GENERAL

Page 4

DECAY CHAIN ACTIVITIES

\begin{tabular}{|c|c|c|c|c|c|}
\hline Nuclide & Stack & Activity at 500. & seconds & Activity at & 100.00 years \\
\hline Cs - 137 & 1 & $8.7600 \mathrm{E}-06$ & & $2.9690 E-06$ & \\
\hline $\mathrm{Ba}-137 \mathrm{~m}$ & 1 & $8.3360 E-06$ & & $2.8070 \mathrm{E}-06$ & \\
\hline Am-241 & 1 & $1.1400 \mathrm{E}-05$ & & $3.8820 E-06$ & \\
\hline$N p-237$ & 1 & $\odot .0000 \mathrm{E}+0 \odot$ & & $\odot .0000 \mathrm{E}+0 \odot$ & \\
\hline $\mathrm{Pa}-233$ & 1 & $\odot .0000 \mathrm{E}+0 \odot$ & & ०. .००००E+०० & \\
\hline$U-233$ & 1 & $\odot .0000 \mathrm{E}+0 \odot$ & & ๑. .०००E+०० & \\
\hline Th-229 & 1 & $\odot .0000 \mathrm{E}+0 \odot$ & & ๑. .०००E+०० & \\
\hline $\mathrm{Ra}-225$ & 1 & $\odot .0000 \mathrm{E}+0 \odot$ & & $\odot .0000 \mathrm{E}+\odot \odot$ & \\
\hline$A c-225$ & 1 & $\odot .0000 \mathrm{E}+0 \odot$ & & ०. .००००E+०० & \\
\hline Fr-221 & 1 & $\odot .0000 \mathrm{E}+0 \odot$ & & ๑. .००००E+०० & \\
\hline At -217 & 1 & $\odot .000 \odot E+\odot \odot$ & & ๑. ००००E+०० & \\
\hline $\mathrm{Bi}-213$ & 1 & $\odot .0000 \mathrm{E}+0 \odot$ & & ०. .००००E+०० & \\
\hline Po- 213 & 1 & $\odot . \odot \odot \odot \odot E+\odot \odot$ & & $\odot . \odot \odot \odot \odot E+\odot \odot$ & \\
\hline $\mathrm{Pb}-209$ & 1 & $\odot . \odot \odot \odot \odot E+\odot \odot$ & & $\odot . \odot \odot \odot \odot E+\odot \odot$ & \\
\hline Tl-209 & 1 & ०. $.000 \mathrm{E}+\odot \odot$ & & ๑. .०००E+०० & \\
\hline $\mathrm{Pu}-238$ & 1 & $8.2000 \mathrm{E}-07$ & & $2.7880 E-07$ & \\
\hline U-234 & 1 & $4.8100 \mathrm{E}-05$ & & 1. 6380E- 05 & \\
\hline Th- 230 & 1 & $4.8100 \mathrm{E}-05$ & & 1. 6380E- 05 & \\
\hline $\mathrm{Ra}-226$ & 1 & $4.8100 E-05$ & & $1.6380 E-05$ & \\
\hline$R n-222$ & 1 & $4.8100 E-05$ & & $1.6370 \mathrm{E}-05$ & \\
\hline Po- 218 & 1 & 4. 810०E- 05 & & $1.6370 E-05$ & \\
\hline $\mathrm{Pb}-214$ & 1 & $4.8100 E-05$ & & $1.6370 \mathrm{E}-05$ & \\
\hline $\mathrm{Bi}-214$ & 1 & $4.8100 E-05$ & & $1.6370 E-05$ & \\
\hline Po- 214 & 1 & $4.8090 E-05$ & & $1.6370 \mathrm{E}-05$ & \\
\hline $\mathrm{Pb}-210$ & 1 & $4.8100 \mathrm{E}-05$ & & 1. 6380E- 05 & \\
\hline $\mathrm{Bi}-210$ & 1 & $4.8100 \mathrm{E}-05$ & & $1.6370 \mathrm{E}-05$ & \\
\hline Po- 210 & 1 & $4.8100 E-05$ & & $1.6380 \mathrm{E}-05$ & \\
\hline At - 218 & 1 & $9.6200 \mathrm{E}-09$ & & $\odot .0 \odot ० 0 \mathrm{E}+\odot \odot$ & \\
\hline Sr - 90 & 1 & $3.1000 \mathrm{E}-06$ & & $1.0500 E-06$ & \\
\hline$Y-90$ & 1 & $3.1000 \mathrm{E}-06$ & & 1. $0500 \mathrm{E}-06$ & \\
\hline$K-40$ & 1 & $2.8800 E-04$ & & $9.8100 \mathrm{E}-05$ & \\
\hline Th-232 & 1 & $2.9600 \mathrm{E}-05$ & & 1. $0080 \mathrm{E}-05$ & \\
\hline $\mathrm{Ra}-228$ & 1 & $2.9600 \mathrm{E}-05$ & & 1. $0080 \mathrm{E}-05$ & \\
\hline$A c-228$ & 1 & $2.9600 \mathrm{E}-05$ & & 1. $0080 \mathrm{E}-05$ & \\
\hline Th- 228 & 1 & $2.9600 \mathrm{E}-05$ & & 1. $0080 \mathrm{E}-05$ & \\
\hline $\mathrm{Ra}-224$ & 1 & $2.9600 \mathrm{E}-05$ & & 1. $0080 \mathrm{E}-05$ & \\
\hline $\mathrm{Rn}-220$ & 1 & $2.9600 \mathrm{E}-05$ & & 1. $0080 \mathrm{E}-05$ & \\
\hline Po- 216 & 1 & $2.9600 \mathrm{E}-05$ & & 1. $0080 \mathrm{E}-05$ & \\
\hline $\mathrm{Pb}-212$ & 1 & $2.9600 \mathrm{E}-05$ & & 1. $0080 \mathrm{E}-05$ & \\
\hline $\mathrm{Bi}-212$ & 1 & $2.9600 \mathrm{E}-05$ & & 1. $0080 \mathrm{E}-05$ & \\
\hline Po- 212 & 1 & $1.8960 E-05$ & & $6.4560 E-06$ & \\
\hline Tl- 208 & 1 & $1.0650 E-05$ & & $3.6200 \mathrm{E}-06$ & \\
\hline$U-238$ & 1 & $4.8100 \mathrm{E}-05$ & & $1.6380 \mathrm{E}-05$ & \\
\hline Th-234 & 1 & $4.8100 \mathrm{E}-05$ & & $1.6380 \mathrm{E}-05$ & \\
\hline $\mathrm{Pa}-234 \mathrm{~m}$ & 1 & $4.8000 E-05$ & & $1.6340 E-05$ & \\
\hline $\mathrm{Pa}-234$ & 1 & $6.2500 \mathrm{E}-08$ & & ๑. .००००E+०० & \\
\hline Al- 26 & 1 & 1. $2800 \mathrm{E}-06$ & & $4.3600 E-07$ & \\
\hline
\end{tabular}




\begin{tabular}{|c|c|c|c|}
\hline $\mathrm{Be}-7$ & 1 & 8. 4490E- - & 1. $2920 \mathrm{E}-06$ \\
\hline $\mathrm{Cm}-243$ & 1 & $6.9400 \mathrm{E}-06$ & $2.3510 \mathrm{E}-06$ \\
\hline Am-243 & 1 & $\odot . \odot \odot \odot \odot E+\odot \odot$ & $\odot . \odot \odot \odot \odot E+\odot \odot$ \\
\hline$N p-239$ & 1 & $\odot . \odot \odot \odot \odot E+\odot \odot$ & $\odot . \odot \odot \odot \odot E+\odot \odot$ \\
\hline Pu-239 & 1 & $3.260 \odot E-\odot 5$ & $1.1100 \mathrm{E}-05$ \\
\hline$U-235$ & 1 & $6.7300 \mathrm{E}-05$ & $2.2920 \mathrm{E}-05$ \\
\hline Th-231 & 1 & $6.7300 \mathrm{E}-05$ & $2.2910 \mathrm{E}-05$ \\
\hline $\mathrm{Pa}-231$ & 1 & $6.7300 \mathrm{E}-\odot 5$ & $2.2920 E-05$ \\
\hline Ac -227 & 1 & $6.7300 \mathrm{E}-05$ & $2.2920 \mathrm{E}-05$ \\
\hline Th- 227 & 1 & $6.6400 \mathrm{E}-05$ & $2.2600 \mathrm{E}-05$ \\
\hline $\mathrm{Ra}-223$ & 1 & $6.7300 \mathrm{E}-05$ & $2.2850 \mathrm{E}-05$ \\
\hline$R n-219$ & 1 & $6.7300 \mathrm{E}-05$ & $2.2850 \mathrm{E}-05$ \\
\hline Po- 215 & 1 & $6.7300 \mathrm{E}-05$ & $2.2850 \mathrm{E}-05$ \\
\hline$P b-211$ & 1 & $6.730 \odot E-\odot 5$ & $2.2850 E-05$ \\
\hline $\mathrm{Bi}-211$ & 1 & $6.7300 \mathrm{E}-05$ & $2.2850 \mathrm{E}-05$ \\
\hline Tl-207 & 1 & $6.7110 \mathrm{E}-05$ & $2.2780 \mathrm{E}-05$ \\
\hline Po- 211 & 1 & $1.884 \odot \mathrm{E}-\odot 7$ & $\odot . \odot \odot \odot \odot E+\odot \odot$ \\
\hline$F r-223$ & 1 & $9.3970 \mathrm{E}-07$ & $3.1610 \mathrm{E}-07$ \\
\hline Co-58 & 1 & $6.40 \odot \odot \mathrm{E}-\odot 7$ & $7.2630 \mathrm{E}-08$ \\
\hline Co-60 & 1 & 1. 2100E-๑6 & $4.0060 \mathrm{E}-07$ \\
\hline Cs -134 & 1 & $5.7600 \mathrm{E}-\odot 6$ & $1.8260 \mathrm{E}-06$ \\
\hline Eu-152 & 1 & $7.1450 E-\odot 6$ & $6.6930 \mathrm{E}-\odot 9$ \\
\hline Gd-152 & 1 & $\odot . \odot \odot \odot \odot E+\odot \odot$ & $\odot . \odot \odot \odot \odot E+\odot \odot$ \\
\hline Eu-154 & 1 & $5.5330 \mathrm{E}-06$ & $2.5710 \mathrm{E}-\odot 9$ \\
\hline Eu-155 & 1 & $3.470 \odot E-\odot 6$ & $1.1620 E-\odot 6$ \\
\hline $\mathrm{Nb}-94$ & 1 & 1. $080 \odot E-\odot 6$ & $3.6790 \mathrm{E}-07$ \\
\hline$S b-125$ & 1 & $2.9100 \mathrm{E}-\odot 6$ & $9.3920 \mathrm{E}-07$ \\
\hline $\mathrm{Te}-125 \mathrm{~m}$ & 1 & $\odot . \odot \odot \odot \odot E+\odot \odot$ & $7.8250 \mathrm{E}-\odot 8$ \\
\hline $\mathrm{H}-3$ & 1 & 1. $30 \odot \odot E-\odot 5$ & $4.3740 E-\odot 6$ \\
\hline
\end{tabular}




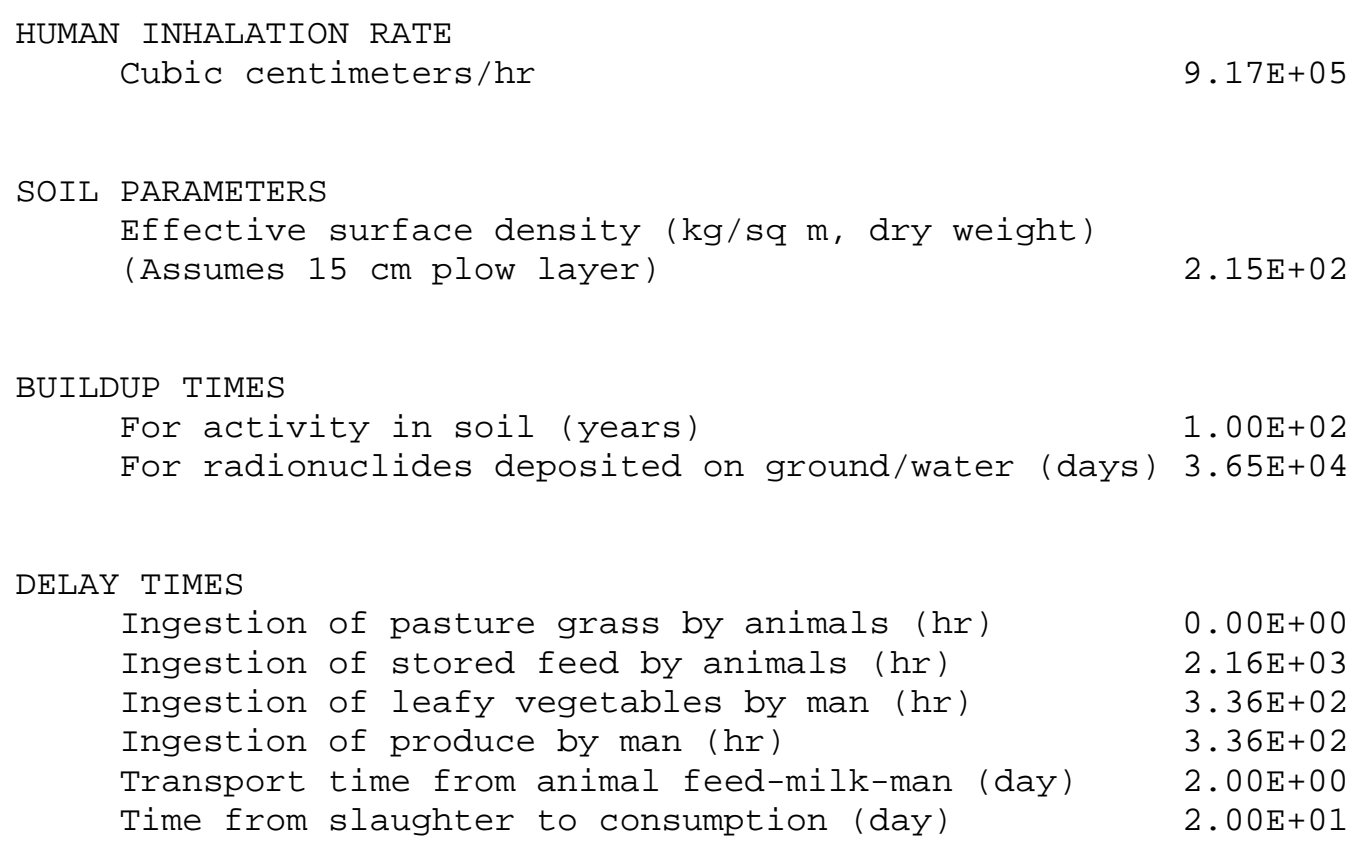


VALUES FOR RADIONUCLIDE-INDEPENDENT PARAMETERS

ANIMAL FEED CONSUMPTION FACTORS

Contaminated feed/forage (kg/day, dry weight) $\quad 1.56 \mathrm{E}+01$

DAIRY PRODUCTIVITY

Milk production of cow (L/day) $1.10 \mathrm{E}+01$

MEAT ANIMAL SLAUGHTER PARAMETERS

Muscle mass of animal at slaughter $(\mathrm{kg}) \quad 2.00 \mathrm{E}+\mathrm{O} 2$

Fraction of herd slaughtered (per day) 3.81E-03

DECONTAMINATION

Fraction of radioactivity retained after washing for leafy vegetables and produce

$5.00 \mathrm{E}-01$

FRACTIONS GROWN IN GARDEN OF INTEREST

Produce ingested

1. $00 \mathrm{E}+\odot \odot$

Leafy vegetables ingested

1. $\odot \odot E+\odot \odot$

INGESTION RATIOS:

IMMEDIATE SURROUNDING AREA/TOTAL WITHIN AREA Vegetables

Meat

Milk

7. $00 \mathrm{E}-01$

4. $40 \mathrm{E}-01$

4. $00 \mathrm{E}-01$

MINIMUM INGESTION FRACTIONS FROM OUTSIDE AREA (Minimum fractions of food types from outside area listed below are actual fixed values.)

Vegetables

Meat

Milk

$\odot .0 \odot \mathrm{E}+\odot \odot$

$\odot . \odot \odot E+\odot \odot$

$\odot . \odot \odot E+\odot \odot$

HUMAN FOOD UTILIZATION FACTORS

Produce ingestion ( $\mathrm{kg} / \mathrm{y})$

1. $76 \mathrm{E}+\odot 2$

Milk ingestion ( $\mathrm{L} / \mathrm{y})$

$1.12 \mathrm{E}+02$

Meat ingestion $(\mathrm{kg} / \mathrm{y})$

8. $50 \mathrm{E}+01$

Leafy vegetable ingestion ( $\mathrm{kg} / \mathrm{y}$ )

$1.80 \mathrm{E}+01$

SWIMMING PARAMETERS

Fraction of time spent swimming $\quad 0.00 \mathrm{E}+\odot \odot$

Dilution factor for water $(\mathrm{cm}) \quad 1.00 \mathrm{E}+\Theta \odot$ 


\author{
C A P 88 - P C \\ Version 3.0 \\ Clean Air Act Assessment Package - 1988
}

\title{
$\begin{array}{llllllllllllll}S & Y & N & O & P & S & I & S & R & E & P & O & R & T\end{array}$ \\ Non-Radon Individual Assessment \\ Nov 27, $2006 \quad 05: 40$ pm
}

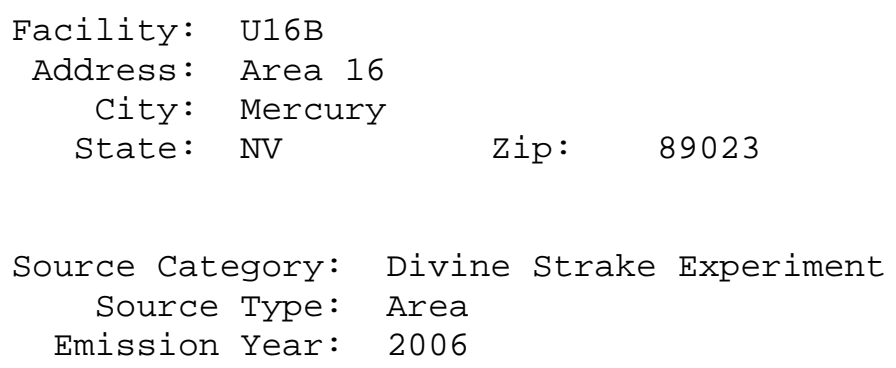

Comments: Dose from the proposed Divine Strake experiment: 2005 average wind conditions used. Source used was maximum upper confidence interval reported in D0E/NV-1177 and total PM10.

Effective Dose Equivalent

( $\mathrm{mrem} /$ year)

\section{1. $06 \mathrm{E}-03$}

At This Location: 22600 Meters East Southeast

Dataset Name: DS SNJV Max UCI

Dataset Date: 10/26/2006 8:58:00 AM

Wind File: C: \Program Files\CAP88-PC30\WindLib\Divine S 
MAXIMALLY EXPOSED INDIVIDUAL

Location of The Individual: 22600 Meters East Southeast

Lifetime Fatal Cancer Risk: 3.42E-10

ORGAN DOSE EQUIVALENT SUMMARY

\begin{tabular}{lc} 
Organ & $\begin{array}{c}\text { Dose } \\
\text { Equivalent } \\
\text { (mrem/y) }\end{array}$ \\
\cline { 2 - 2 } Adrenals & $2.83 \mathrm{E}-05$ \\
B Surfac & $3.15 \mathrm{E}-05$ \\
Breasts & $1.56 \mathrm{E}-03$ \\
St Wall & $2.92 \mathrm{E}-05$ \\
ULI Wall & $3.17 \mathrm{E}-05$ \\
Kidneys & $2.76 \mathrm{E}-05$ \\
Lungs & $3.02 \mathrm{E}-05$ \\
Ovaries & $2.95 \mathrm{E}-05$ \\
R Marrow & $3.41 \mathrm{E}-05$ \\
Spleen & $4.33 \mathrm{E}-05$ \\
Thymus & $4.59 \mathrm{E}-05$ \\
Uterus & $1.56 \mathrm{E}-04$ \\
Bld Wall & $1.58 \mathrm{E}-04$ \\
Brain & $3.25 \mathrm{E}-05$ \\
Esophagu & $4.30 \mathrm{E}-05$ \\
SI Wall & $2.85 \mathrm{E}-05$ \\
LLI Wall & $9.30 \mathrm{E}-05$ \\
Liver & $3.78 \mathrm{E}-04$ \\
Muscle & $3.17 \mathrm{E}-05$ \\
Pancreas & $4.66 \mathrm{E}-05$ \\
Skin & $2.95 \mathrm{E}-05$ \\
Testes & $3.05 \mathrm{E}-05$ \\
Thyroid & $2.92 \mathrm{E}-05$ \\
& \\
EFFEC & $1.06 \mathrm{E}-03$
\end{tabular}


RADIONUCLIDE EMISSIONS DURING THE YEAR 2006

\begin{tabular}{|c|c|c|c|c|}
\hline Nuclide & Type & Size & $\begin{array}{c}\text { Source } \\
\# 1 \\
\mathrm{Ci} / \mathrm{y}\end{array}$ & $\begin{array}{r}\text { TOTAL } \\
\mathrm{Ci} / \mathrm{y}\end{array}$ \\
\hline Cs -137 & $\mathrm{~F}$ & 1 & $8.8 \mathrm{E}-06$ & $8.8 \mathrm{E}-06$ \\
\hline $\mathrm{Ba}-137 \mathrm{~m}$ & M & 1 & $8.8 \mathrm{E}-06$ & $8.8 E-06$ \\
\hline Am- 241 & M & 1 & 1.1E- 05 & 1. 1E- 05 \\
\hline $\mathrm{Pu}-238$ & M & 1 & $8.2 \mathrm{E}-07$ & $8.2 \mathrm{E}-07$ \\
\hline Pu-239 & M & 1 & $3.3 E-05$ & $3.3 E-05$ \\
\hline $\mathrm{Sr}-90$ & M & 1 & $3.1 \mathrm{E}-06$ & $3.1 \mathrm{E}-06$ \\
\hline Y - $9 \odot$ & M & 1 & $3.1 \mathrm{E}-06$ & $3.1 \mathrm{E}-06$ \\
\hline$K-4 \odot$ & M & 1 & $2.9 \mathrm{E}-\odot 4$ & $2.9 E-\odot 4$ \\
\hline Th- 232 & $S$ & 1 & $3.0 \mathrm{E}-05$ & 3. $0 \mathrm{E}-05$ \\
\hline $\mathrm{Ra}-228$ & M & 1 & $3 . \odot E-\odot 5$ & 3. $0 \mathrm{E}-\odot 5$ \\
\hline$A C-228$ & $M$ & 1 & $3.0 \mathrm{E}-05$ & $3.0 \mathrm{E}-05$ \\
\hline Th-228 & $\mathrm{s}$ & 1 & $3.0 \mathrm{E}-05$ & $3.0 \mathrm{E}-05$ \\
\hline $\mathrm{Ra}-224$ & M & 1 & $3.0 \mathrm{E}-\odot 5$ & 3. $\odot E-\odot 5$ \\
\hline $\mathrm{Rn}-220$ & G & $\odot$ & $3.0 \mathrm{E}-05$ & $3.0 \mathrm{E}-05$ \\
\hline Po-216 & M & 1 & $3.0 \mathrm{E}-05$ & $3.0 \mathrm{E}-05$ \\
\hline $\mathrm{Pb}-212$ & M & 1 & $3.0 E-05$ & 3. $\odot E-\odot 5$ \\
\hline $\mathrm{Bi}-212$ & $M$ & 1 & $3.0 \mathrm{E}-05$ & $3.0 \mathrm{E}-05$ \\
\hline Po- 212 & M & 1 & $1.9 \mathrm{E}-05$ & 1. $9 \mathrm{E}-05$ \\
\hline Tl-208 & M & 1 & $1.1 \mathrm{E}-05$ & 1. $1 \mathrm{E}-05$ \\
\hline$U-238$ & M & 1 & $4.8 \mathrm{E}-05$ & $4.8 E-05$ \\
\hline Th-234 & S & 1 & $4.8 \mathrm{E}-05$ & $4.8 \mathrm{E}-05$ \\
\hline $\mathrm{Pa}-234 \mathrm{~m}$ & M & 1 & $4.8 \mathrm{E}-05$ & $4.8 E-05$ \\
\hline $\mathrm{Pa}-234$ & $M$ & 1 & $6.2 \mathrm{E}-08$ & $6.2 \mathrm{E}-08$ \\
\hline$U-234$ & $M$ & 1 & $4.8 \mathrm{E}-05$ & $4.8 E-05$ \\
\hline Th-230 & S & 1 & $4.8 \mathrm{E}-05$ & $4.8 E-05$ \\
\hline $\mathrm{Ra}-226$ & $M$ & 1 & $4.8 E-05$ & $4.8 E-05$ \\
\hline$R n-222$ & G & $\odot$ & $4.8 \mathrm{E}-05$ & $4.8 E-05$ \\
\hline Po- 218 & M & 1 & $4.8 \mathrm{E}-05$ & $4.8 \mathrm{E}-05$ \\
\hline $\mathrm{Pb}-214$ & M & 1 & $4.8 \mathrm{E}-05$ & $4.8 \mathrm{E}-05$ \\
\hline At -218 & M & 1 & $9.6 \mathrm{E}-09$ & $9.6 \mathrm{E}-\odot 9$ \\
\hline Bi-214 & M & 1 & $4.8 \mathrm{E}-05$ & $4.8 \mathrm{E}-05$ \\
\hline Po-214 & M & 1 & $4.8 \mathrm{E}-05$ & $4.8 \mathrm{E}-05$ \\
\hline $\mathrm{Pb}-210$ & M & 1 & $4.8 \mathrm{E}-05$ & 4. $8 \mathrm{E}-05$ \\
\hline $\mathrm{Bi}-210$ & $M$ & 1 & $4.8 \mathrm{E}-05$ & $4.8 \mathrm{E}-05$ \\
\hline Po-210 & M & 1 & $4.8 \mathrm{E}-05$ & $4.8 \mathrm{E}-05$ \\
\hline U-235 & M & 1 & $6.7 \mathrm{E}-05$ & $6.7 \mathrm{E}-05$ \\
\hline Th-231 & $\mathrm{s}$ & 1 & $6.7 \mathrm{E}-05$ & $6.7 \mathrm{E}-05$ \\
\hline $\mathrm{Pa}-231$ & $M$ & 1 & 6. 7E- -55 & $6.7 \mathrm{E}-05$ \\
\hline$A c-227$ & M & 1 & $6.7 \mathrm{E}-05$ & $6.7 \mathrm{E}-05$ \\
\hline Th -227 & $\mathrm{~s}$ & 1 & $6.6 \mathrm{E}-05$ & $6.6 \mathrm{E}-05$ \\
\hline $\mathrm{Fr}-223$ & M & 1 & $9.4 \mathrm{E}-07$ & $9.4 \mathrm{E}-07$ \\
\hline $\mathrm{Ra}-223$ & M & 1 & $6.7 \mathrm{E}-05$ & $6.7 \mathrm{E}-05$ \\
\hline $\mathrm{Rn}-219$ & G & 0 & $6.7 \mathrm{E}-05$ & $6.7 \mathrm{E}-05$ \\
\hline Po- 215 & $M$ & 1 & 6. $7 \mathrm{E}-05$ & $6.7 \mathrm{E}-05$ \\
\hline $\mathrm{Pb}-211$ & M & 1 & $6.7 \mathrm{E}-05$ & $6.7 \mathrm{E}-05$ \\
\hline
\end{tabular}




$\begin{array}{lllll}\mathrm{Bi}-211 & \mathrm{M} & 1 & 6.7 \mathrm{E}-05 & 6.7 \mathrm{E}-05 \\ \mathrm{Tl}-207 & \mathrm{M} & 1 & 6.7 \mathrm{E}-05 & 6.7 \mathrm{E}-05 \\ \mathrm{Po}-211 & \mathrm{M} & 1 & 1.9 \mathrm{E}-07 & 1.9 \mathrm{E}-07 \\ \mathrm{Al}-26 & \mathrm{M} & 1 & 1.3 \mathrm{E}-06 & 1.3 \mathrm{E}-06 \\ \mathrm{Be}-7 & \mathrm{M} & 1 & 8.4 \mathrm{E}-06 & 8.4 \mathrm{E}-06 \\ \mathrm{Cm}-243 & \mathrm{M} & 1 & 6.9 \mathrm{E}-06 & 6.9 \mathrm{E}-06 \\ \mathrm{Co}-58 & \mathrm{M} & 1 & 6.4 \mathrm{E}-07 & 6.4 \mathrm{E}-07 \\ \mathrm{Co}-60 & \mathrm{M} & 1 & 1.2 \mathrm{E}-06 & 1.2 \mathrm{E}-06 \\ \mathrm{Cs}-134 & \mathrm{~F} & 1 & 5.8 \mathrm{E}-06 & 5.8 \mathrm{E}-06 \\ \mathrm{Eu}-152 & \mathrm{M} & 1 & 7.2 \mathrm{E}-06 & 7.2 \mathrm{E}-06 \\ \mathrm{Eu}-154 & \mathrm{M} & 1 & 5.6 \mathrm{E}-06 & 5.6 \mathrm{E}-06 \\ \mathrm{Eu}-155 & \mathrm{M} & 1 & 3.5 \mathrm{E}-06 & 3.5 \mathrm{E}-06 \\ \mathrm{Nb}-94 & \mathrm{M} & 1 & 1.1 \mathrm{E}-06 & 1.1 \mathrm{E}-06 \\ \mathrm{Sb}-125 & \mathrm{M} & 1 & 2.9 \mathrm{E}-06 & 2.9 \mathrm{E}-06 \\ \mathrm{H}-3 & \mathrm{~V} & \odot & 1.3 \mathrm{E}-05 & 1.3 \mathrm{E}-05\end{array}$

\section{SITE INFORMATION}

Temperature: 13 degrees $\mathrm{C}$ Precipitation: $\quad 3 \mathrm{~cm} / \mathrm{y}$

Humidity: $\quad 3 \mathrm{~g} / \mathrm{cu} \mathrm{m}$ Mixing Height: $3000 \mathrm{~m}$ 


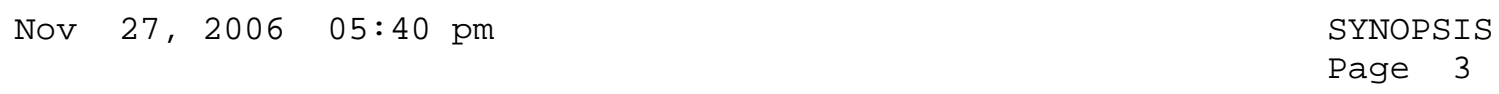

SOURCE INFORMATION

Source Number: 1

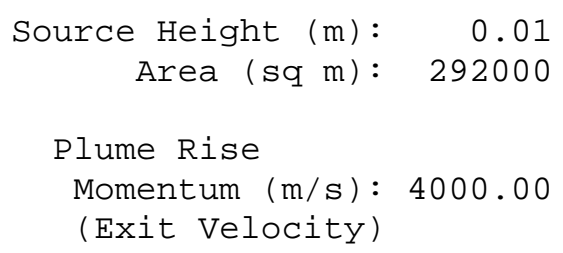

AGRICULTURAL DATA

\begin{tabular}{|c|c|c|c|}
\hline & Vegetable & Milk & Meat \\
\hline Fraction Home Produced: & 0.700 & 0.400 & 0.440 \\
\hline Fraction From Assessment Area: & 0.300 & 0.600 & 0.560 \\
\hline Fraction Imported: & 0.000 & 0.000 & 0.000 \\
\hline
\end{tabular}

Food Arrays were not generated for this run. Default values used.

DISTANCES (M) USED FOR MAXIMUM INDIVIDUAL ASSESSMENT

$\begin{array}{llllllllll}22600 & 46710 & 48940 & 49330 & 51120 & 53270 & 54360 & 58780 & 59110 & 61710 \\ 63600 & 67530 & 69590 & 70340 & 73520 & 75750 & 76380 & 78440 & 79130 & 79450\end{array}$




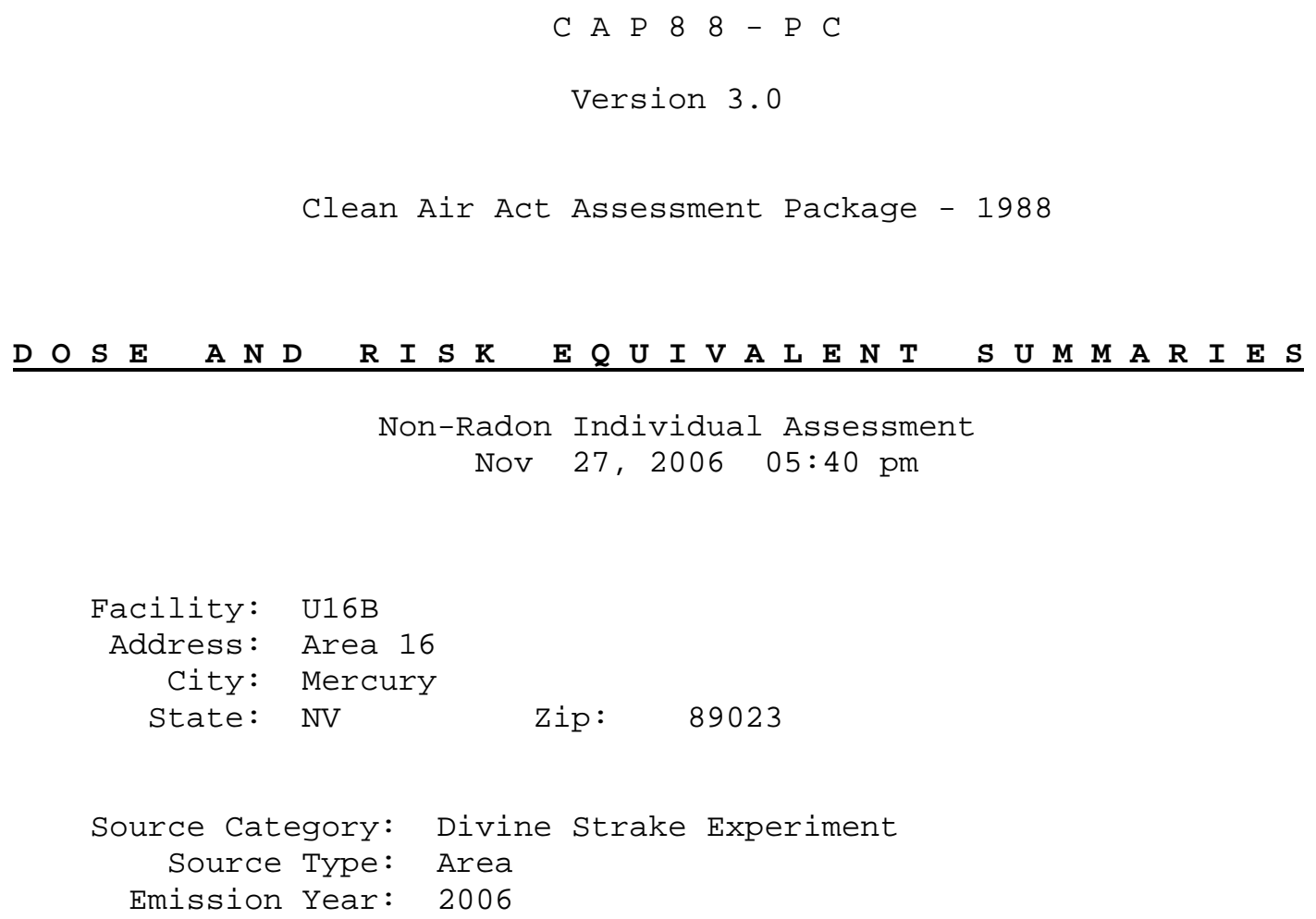

Comments: Dose from the proposed Divine Strake experiment: 2005 average wind conditions used. Source used was maximum upper confidence interval reported in DOE/NV-1177 and total PM10.

Dataset Name: DS SNJV Max UCI

Dataset Date: 10/26/2006 8:58:00 AM

Stake \05MEDA21. WND

Wind File: . C: \Program Files\CAP88-PC30\WindLib\Divine 
Appendix A

Nov 27, $2006 \quad 05: 40$ pm

SUMMARY

Page 1

ORGAN DOSE EQUIVALENT SUMMARY

\begin{tabular}{|c|c|}
\hline Organ & $\begin{array}{l}\text { Selected } \\
\text { Individual } \\
(\text { mrem/y) }\end{array}$ \\
\hline Adrenals & $2.83 E-05$ \\
\hline B Surfac & $3.15 \mathrm{E}-05$ \\
\hline Breasts & $1.56 \mathrm{E}-03$ \\
\hline St wall & $2.92 \mathrm{E}-05$ \\
\hline ULI Wall & $3.17 \mathrm{E}-05$ \\
\hline Kidneys & $2.76 \mathrm{E}-05$ \\
\hline Lungs & $3.02 \mathrm{E}-05$ \\
\hline Ovaries & $2.95 \mathrm{E}-05$ \\
\hline $\mathrm{R}$ Marrow & $3.41 \mathrm{E}-05$ \\
\hline spleen & $4.33 E-05$ \\
\hline Thymus & $4.59 \mathrm{E}-05$ \\
\hline Uterus & $1.56 \mathrm{E}-04$ \\
\hline Bld Wall & $1.58 \mathrm{E}-04$ \\
\hline Brain & $3.25 \mathrm{E}-05$ \\
\hline Esophagu & $4.30 E-05$ \\
\hline SI Wall & $2.85 \mathrm{E}-05$ \\
\hline LLI Wall & $9.30 \mathrm{E}-05$ \\
\hline Liver & $3.78 \mathrm{E}-\odot 4$ \\
\hline Muscle & $3.17 \mathrm{E}-05$ \\
\hline Pancreas & $4.66 \mathrm{E}-05$ \\
\hline Skin & $2.95 \mathrm{E}-05$ \\
\hline Testes & $3.05 \mathrm{E}-05$ \\
\hline Thyroid & $2.92 \mathrm{E}-05$ \\
\hline EFFEC & 1. $06 \mathrm{E}-03$ \\
\hline
\end{tabular}

PATHWAY EFFECTIVE DOSE EQUIVALENT SUMMARY

Pathway

INGESTION

INHALATION

AIR IMMERSION

GROUND SURFACE

INTERNAL

EXTERNAL

TOTAL
Selected

Individual

$(\mathrm{mrem} / \mathrm{y})$

$3.22 \mathrm{E}-05$

$9.98 \mathrm{E}-04$

$3.00 \mathrm{E}-09$

2. 55E- 05

1. $03 \mathrm{E}-03$

2. 55E- 05

1. $06 \mathrm{E}-03$ 
Nov $27,2006 \quad 05: 40 \mathrm{pm}$

SUMMARY

Page 2

NUCLIDE EFFECTIVE DOSE EQUIVALENT SUMMARY

Nuclide

Cs -137

$\mathrm{Ba}-137 \mathrm{~m}$

Am- 241

$\mathrm{Np}-237$

$\mathrm{Pa}-233$

U-233

Th- 229

$\mathrm{Ra}-225$

Ac- -225

Fr- 221

At -217

$\mathrm{Bi}-213$

Po- 213

$\mathrm{Pb}-209$

Tl-209

$\mathrm{Pu}-238$

U-234

Th- 230

$\mathrm{Ra}-226$

$\mathrm{Rn}-222$

Po- 218

$\mathrm{Pb}-214$

Bi-214

Po- 214

$\mathrm{Pb}-210$

Bi -210

Po- 210

At -218

Sr -90

$\mathrm{Y}-90$

$\mathrm{K}-40$

Th-232

Ra-228

Ac- 228

Th-228

Ra-224

$\mathrm{Rn}-220$

Po-216

$\mathrm{Pb}-212$

Bi-212

Po- 212

T1-208

U-238

Th-234
Selected

Individual

(mrem/y)

2. 71E- 07

$4.32 \mathrm{E}-07$

2. $61 \mathrm{E}-05$

๑. . O०E+००

$\odot . ~ . \odot E+\odot \odot$

๑ . ००E+००

$\odot . ~ \odot \odot E+\odot \odot$

๑ . ००E+००

๑ . ००E+०९

$\odot . ~ . \odot E+\odot \odot$

๑ . ००E+००

๑ . ००E+००

$\odot . ~ \odot \odot E+\odot \odot$

$\odot . ~ . \odot E+\odot \odot$

$\odot . \odot \odot E+\odot \odot$

2. $07 \mathrm{E}-06$

$9.36 E-06$

3. 76E- 05

1. $28 \mathrm{E}-05$

6. $85 \mathrm{E}-13$

3. $76 \mathrm{E}-11$

1. . $08 E-06$

6. 31E- 06

3. $45 \mathrm{E}-10$

7. . $04 \mathrm{E}-06$

3. $99 \mathrm{E}-07$

9. $91 \mathrm{E}-06$

2. $00 \mathrm{E}-16$

1. $88 \mathrm{E}-07$

3. 10E- 08

1. $05 E-05$

4. $05 E-05$

9. $95 \mathrm{E}-06$

2. 54E- 06

6. 39E- 05

4. 82E- $\odot 6$

4. $07 \mathrm{E}-13$

$4.32 \mathrm{E}-11$

6. 38E- 07

$6.53 \mathrm{E}-07$

$\odot . \odot \odot E+\odot \odot$

2. 86E- $\odot 6$

7. 71E-06

$6.11 E-08$ 


\begin{tabular}{|c|c|}
\hline $\mathrm{Pa}-234 \mathrm{~m}$ & $4.7 \odot E-\odot 7$ \\
\hline $\mathrm{Pa}-234$ & $2.48 \mathrm{E}-12$ \\
\hline Al-26 & $2.88 \mathrm{E}-07$ \\
\hline $\mathrm{Be}-7$ & $1.63 \mathrm{E}-08$ \\
\hline $\mathrm{Cm}-243$ & $1.20 \mathrm{E}-\odot 5$ \\
\hline Am-243 & $\odot . \odot \odot E+\odot \odot$ \\
\hline$N p-239$ & $\odot . \odot \odot \mathrm{E}+\odot \odot$ \\
\hline Pu-239 & $8.95 \mathrm{E}-05$ \\
\hline$U-235$ & $1.25 \mathrm{E}-05$ \\
\hline Th-231 & $9.63 E-\odot 8$ \\
\hline $\mathrm{Pa}-231$ & $3.46 \mathrm{E}-\odot 4$ \\
\hline Ac -227 & $2.68 \mathrm{E}-04$ \\
\hline Th-227 & $3.80 E-05$ \\
\hline $\mathrm{Ra}-223$ & $2.85 \mathrm{E}-05$ \\
\hline$R n-219$ & 1. $32 \mathrm{E}-10$ \\
\hline Po-215 & 1. $\odot 2 E-\odot 9$ \\
\hline$P b-211$ & $6.17 \mathrm{E}-\odot 7$ \\
\hline Bi-211 & $2.68 \mathrm{E}-\odot 7$ \\
\hline Tl-207 & $3.37 E-07$ \\
\hline Po-211 & $1.43 \mathrm{E}-14$ \\
\hline $\mathrm{Fr}-223$ & $7.06 \mathrm{E}-09$ \\
\hline Сo-58 & $1.80 \mathrm{E}-08$ \\
\hline Co- $6 \odot$ & $2.52 \mathrm{E}-07$ \\
\hline Cs -134 & $9.41 \mathrm{E}-07$ \\
\hline Eu-152 & $1.83 E-08$ \\
\hline Gd-152 & $\odot . \odot \odot E+\odot \odot$ \\
\hline Eu-154 & $1.68 E-08$ \\
\hline Eu-155 & $1.80 \mathrm{E}-08$ \\
\hline $\mathrm{Nb}-94$ & $1.47 \mathrm{E}-07$ \\
\hline Sb-125 & $1.03 E-07$ \\
\hline $\mathrm{Te}-125 \mathrm{~m}$ & $6.51 \mathrm{E}-10$ \\
\hline $\mathrm{H}-3$ & $3.00 \mathrm{E}-10$ \\
\hline TOTAL & 1.06E-03 \\
\hline
\end{tabular}


CANCER RISK SUMMARY

\begin{tabular}{lc} 
& $\begin{array}{c}\text { Selected Individual } \\
\text { Total Lifetime } \\
\text { Fatal Cancer Risk }\end{array}$ \\
\cline { 2 - 2 } Esophagu & $8.86 \mathrm{E}-13$ \\
Stomach & $2.56 \mathrm{E}-12$ \\
Colon & $8.52 \mathrm{E}-12$ \\
Liver & $2.94 \mathrm{E}-11$ \\
LUNG & $2.59 \mathrm{E}-10$ \\
Bone & $1.73 \mathrm{E}-11$ \\
Skin & $4.15 \mathrm{E}-13$ \\
Breast & $2.21 \mathrm{E}-12$ \\
Ovary & $3.59 \mathrm{E}-12$ \\
Bladder & $2.13 \mathrm{E}-12$ \\
Kidneys & $1.85 \mathrm{E}-12$ \\
Thyroid & $2.05 \mathrm{E}-13$ \\
Leukemia & $4.75 \mathrm{E}-12$ \\
Residual & $9.39 \mathrm{E}-12$ \\
Total & $3.42 \mathrm{E}-10$ \\
TotaL & $6.84 \mathrm{E}-10$ \\
&
\end{tabular}

PATHWAY RISK SUMMARY

Pathway

INGESTION

INHALATION

AIR IMMERSION

GROUND SURFACE

INTERNAL

EXTERNAL

TOTAL
Selected Individual Total Lifetime Fatal Cancer Risk

1. $25 \mathrm{E}-11$

$3.17 \mathrm{E}-10$

1. $62 \mathrm{E}-15$

1. $25 \mathrm{E}-11$

3. $29 \mathrm{E}-10$

1. $25 \mathrm{E}-11$

$3.42 \mathrm{E}-10$ 
Appendix A

Nov $27,2006 \quad 05: 40$ pm

SUMMARY

Page 4

NUCLIDE RISK SUMMARY

Nuclide

Cs -137

Ba-137m

Am- 241

$\mathrm{Np}-237$

$\mathrm{Pa}-233$

$\mathrm{U}-233$

Th- 229

$\mathrm{Ra}-225$

Ac -225

Fr -221

At -217

$\mathrm{Bi}-213$

Po-213

$\mathrm{Pb}-209$

Tl- 209

$\mathrm{Pu}-238$

$\mathrm{U}-234$

Th -230

$\mathrm{Ra}-226$

$\mathrm{Rn}-222$

Po- 218

$\mathrm{Pb}-214$

Bi- 214

Po- 214

$\mathrm{Pb}-210$

$\mathrm{Bi}-210$

Po- 210

At -218

Sr -90

$\mathrm{Y}-90$

$\mathrm{K}-40$

Th- 232

$\mathrm{Ra}-228$

Ac -228

Th- 228

$\mathrm{Ra}-224$

$\mathrm{Rn}-220$

Po- 216

$\mathrm{Pb}-212$

Bi-212

Po- 212

$\mathrm{T} 1-208$

U-238

Th -234
Selected Individual

Total Lifetime

Fatal Cancer Risk

1. $37 \mathrm{E}-13$

2. $33 \mathrm{E}-13$

$4.12 \mathrm{E}-12$

$\odot . ~ .0 \mathrm{E}+0 \odot$

$\odot ., \odot E+\odot \odot$

$\odot ., 0 \odot \mathrm{E}+\odot \odot$

$\odot . \odot \odot E+\odot \odot$

$\odot ., \odot E+\odot \odot$

$\odot . ~ \odot \odot E+\odot \odot$

$\odot . ~ \odot \odot E+\odot \odot$

$\odot . \odot \odot E+\odot \odot$

$\odot . ~ . \odot E+\odot \odot$

$\odot ., 0 \mathrm{E}+0 \odot$

$\odot . ~ . ० E+\odot \odot$

$\odot . ~ . \odot E+\odot \odot$

3. $61 \mathrm{E}-13$

7. $66 \mathrm{E}-12$

1. $90 \mathrm{E}-11$

8. $95 \mathrm{E}-12$

3. $72 \mathrm{E}-19$

2. $06 \mathrm{E}-17$

$5.82 E-13$

3. $35 \mathrm{E}-12$

1. $89 \mathrm{E}-16$

3. $17 \mathrm{E}-12$

2. 34E-13

7. $73 \mathrm{E}-12$

$9.70 \mathrm{E}-23$

1. $11 \mathrm{E}-13$

3. $92 \mathrm{E}-15$

7. $21 \mathrm{E}-12$

1. $77 \mathrm{E}-11$

4. $27 \mathrm{E}-12$

1. 35E- 12

5. $47 \mathrm{E}-11$

4. 14E-12

2. $23 E-19$

2. $37 \mathrm{E}-17$

4. $35 \mathrm{E}-13$

$3.03 E-13$

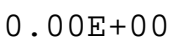

1. 56E-12

6. 30E-12

$4.78 \mathrm{E}-14$ 
Appendix A

$\mathrm{Pa}-234 \mathrm{~m}$

$\mathrm{Pa}-234$

Al-26

Be- 7

Cm- 243

Am- 243

$\mathrm{Np}-239$

$\mathrm{Pu}-239$

U-235

Th-231

$\mathrm{Pa}-231$

Ac -227

Th-227

Ra-223

Rn-219

Po- 215

$\mathrm{Pb}-211$

Bi-211

Tl-207

Po-211

$\mathrm{Fr}-223$

Co-58

Co- 60

Cs-134

Eu-152

Gd-152

Eu-154

Eu-155

$\mathrm{Nb}-94$

$\mathrm{Sb}-125$

Te-125m

H-3

TOTAL
7. 52E-14

1. $58 \mathrm{E}-18$

1. $57 \mathrm{E}-13$

8. $90 \mathrm{E}-15$

2. $48 \mathrm{E}-12$

$\odot .00 \mathrm{E}+\odot \odot$

$\odot .0 \odot \mathrm{E}+\odot \odot$

1. $42 \mathrm{E}-11$

9. $97 \mathrm{E}-12$

4. $42 \mathrm{E}-14$

3. $28 \mathrm{E}-11$

7. $05 \mathrm{E}-11$

3. $28 \mathrm{E}-11$

2. $42 \mathrm{E}-11$

7.16E-17

5. $60 \mathrm{E}-16$

2. $26 \mathrm{E}-13$

1. $47 \mathrm{E}-13$

4. $30 \mathrm{E}-14$

7. 81E-21

2. $92 \mathrm{E}-15$

9. $98 \mathrm{E}-15$

1. $43 \mathrm{E}-13$

5. $05 \mathrm{E}-13$

8. $92 \mathrm{E}-15$

$\odot .00 \mathrm{E}+\odot \odot$

8. 43E-15

9. $54 \mathrm{E}-15$

8. $08 \mathrm{E}-14$

5. 65E-14

2. $97 \mathrm{E}-16$

1. $85 \mathrm{E}-16$

$3.42 \mathrm{E}-10$ 
Nov 27, $2006 \quad 05: 40$ pm

SUMMARY

Page 5

INDIVIDUAL EFFECTIVE DOSE EQUIVALENT RATE ( $\mathrm{mrem} / \mathrm{y}$ )

(All Radionuclides and Pathways)

\begin{tabular}{|c|c|c|c|c|c|c|c|}
\hline \multirow[b]{2}{*}{ Direction } & \multicolumn{7}{|c|}{ Distance $(\mathrm{m})$} \\
\hline & 22600 & 46710 & 48940 & 49330 & 51120 & 53270 & 54360 \\
\hline $\mathrm{N}$ & $3.5 \mathrm{E}-\odot 4$ & $1.1 \mathrm{E}-04$ & 1. $0 \mathrm{E}-\odot 4$ & $9.9 \mathrm{E}-05$ & $9.3 E-05$ & $8.7 \mathrm{E}-05$ & $8.3 E-05$ \\
\hline NNW & 1. $7 \mathrm{E}-\odot 4$ & $4.9 E-\odot 5$ & $4.6 E-05$ & $4.6 E-\odot 5$ & 4. 3E- $\odot 5$ & 4. ๑E-๑5 & $3.9 E-05$ \\
\hline NW & 1. $3 E-04$ & $3.9 E-05$ & $3.7 \mathrm{E}-05$ & $3.6 \mathrm{E}-05$ & $3.4 \mathrm{E}-\odot 5$ & $3.2 \mathrm{E}-\odot 5$ & $3.1 \mathrm{E}-05$ \\
\hline WNW & 1. 3E-๑4 & $4.2 \mathrm{E}-05$ & $3.9 \mathrm{E}-05$ & $3.9 E-05$ & $3.7 \mathrm{E}-05$ & $3.4 \mathrm{E}-05$ & $3.3 E-05$ \\
\hline W & $2.9 \mathrm{E}-\odot 4$ & $8.5 E-\odot 5$ & $7.9 E-\odot 5$ & $7.8 \mathrm{E}-\odot 5$ & $7.4 \mathrm{E}-\odot 5$ & $6.8 \mathrm{E}-\odot 5$ & $6.6 \mathrm{E}-05$ \\
\hline WSW & $3.3 E-04$ & $9.8 \mathrm{E}-\odot 5$ & $9.1 \mathrm{E}-05$ & 9. ०E-๑5 & $8.5 E-\odot 5$ & $7.8 \mathrm{E}-\odot 5$ & $7.5 E-\odot 5$ \\
\hline SW & $3.5 E-04$ & $9.8 \mathrm{E}-05$ & $9.1 \mathrm{E}-05$ & $8.9 E-05$ & $8.4 \mathrm{E}-05$ & $7.8 E-05$ & $7.4 \mathrm{E}-05$ \\
\hline SSW & 1. $3 E-\odot 4$ & $3.6 E-05$ & $3.3 E-\odot 5$ & $3.3 E-\odot 5$ & $3.1 \mathrm{E}-05$ & $2.8 E-\odot 5$ & $2.7 \mathrm{E}-\odot 5$ \\
\hline $\mathrm{S}$ & $1.8 \mathrm{E}-04$ & $5.2 \mathrm{E}-\odot 5$ & $4.8 E-05$ & $4.7 E-05$ & $4.4 \mathrm{E}-05$ & $4.1 \mathrm{E}-05$ & $3.9 \mathrm{E}-\odot 5$ \\
\hline SSE & $2.3 E-04$ & $5.8 \mathrm{E}-05$ & $5.4 \mathrm{E}-05$ & $5.3 E-05$ & $5 . \odot E-\odot 5$ & $4.7 \mathrm{E}-05$ & $4.5 E-05$ \\
\hline SE & $7.3 E-\odot 4$ & $2.1 \mathrm{E}-\odot 4$ & $2 . \odot E-\odot 4$ & 1. $9 \mathrm{E}-\odot 4$ & $1.8 \mathrm{E}-\odot 4$ & $1.7 \mathrm{E}-\odot 4$ & $1.6 \mathrm{E}-\odot 4$ \\
\hline ESE & $1.1 \mathrm{E}-03$ & $3.4 \mathrm{E}-\odot 4$ & $3.1 \mathrm{E}-04$ & $3.1 \mathrm{E}-04$ & $2.9 \mathrm{E}-\odot 4$ & $2.7 \mathrm{E}-\odot 4$ & $2.6 \mathrm{E}-\odot 4$ \\
\hline$E$ & $2.8 \mathrm{E}-04$ & $8.7 \mathrm{E}-05$ & $8.1 \mathrm{E}-05$ & $8.0 \mathrm{E}-05$ & $7.6 \mathrm{E}-05$ & $7.1 \mathrm{E}-05$ & $6.8 \mathrm{E}-05$ \\
\hline ENE & $1.6 \mathrm{E}-\odot 4$ & $4.8 E-\odot 5$ & $4.5 E-05$ & 4. 4E- $\odot 5$ & $4.2 \mathrm{E}-\odot 5$ & $3.9 \mathrm{E}-05$ & $3.7 \mathrm{E}-05$ \\
\hline $\mathrm{NE}$ & $2.3 E-04$ & 7. $0 \mathrm{E}-\odot 5$ & $6.5 \mathrm{E}-05$ & $6.5 \mathrm{E}-05$ & $6.1 \mathrm{E}-05$ & $5.7 \mathrm{E}-05$ & $5.5 E-\odot 5$ \\
\hline NNE & $3.1 \mathrm{E}-04$ & 1. $0 \mathrm{E}-\odot 4$ & $9.5 \mathrm{E}-05$ & $9.4 \mathrm{E}-05$ & $8.9 E-05$ & $8.3 E-05$ & 8. $0 \mathrm{E}-05$ \\
\hline
\end{tabular}

Distance $(\mathrm{m})$

\begin{tabular}{|c|c|c|c|c|c|c|c|}
\hline Direction & 58780 & 59110 & 61710 & 63600 & 67530 & 69590 & 70340 \\
\hline $\mathrm{N}$ & $6.8 \mathrm{E}-\odot 5$ & $6.7 \mathrm{E}-\odot 5$ & $5.4 \mathrm{E}-\odot 5$ & $5.2 E-05$ & $4.8 E-05$ & $4.6 \mathrm{E}-\odot 5$ & $4.5 E-05$ \\
\hline NNW & $3.2 \mathrm{E}-05$ & $3.2 \mathrm{E}-05$ & $2.6 \mathrm{E}-05$ & $2.5 \mathrm{E}-05$ & $2.3 E-\odot 5$ & $2.2 \mathrm{E}-05$ & $2.2 \mathrm{E}-05$ \\
\hline NW & $2.6 E-05$ & $2.5 E-05$ & 2. $\odot \mathrm{E}-\odot 5$ & $1.9 E-05$ & $1.8 \mathrm{E}-05$ & 1.7E- 05 & 1. $7 \mathrm{E}-05$ \\
\hline WNW & $2.7 \mathrm{E}-\odot 5$ & $2.7 \mathrm{E}-\odot 5$ & $2.1 \mathrm{E}-05$ & $2.1 \mathrm{E}-05$ & 1. $9 \mathrm{E}-\odot 5$ & $1.8 \mathrm{E}-\odot 5$ & $1.8 \mathrm{E}-05$ \\
\hline W & $5.2 \mathrm{E}-05$ & $5 . \odot E-\odot 5$ & $3.7 \mathrm{E}-05$ & $3.5 \mathrm{E}-05$ & $3.3 E-05$ & $3.1 \mathrm{E}-05$ & $3.1 \mathrm{E}-05$ \\
\hline WSW & 6. ๑E-๑5 & $5.8 E-\odot 5$ & $4.3 E-\odot 5$ & $4.1 E-\odot 5$ & $3.8 E-\odot 5$ & $3.7 \mathrm{E}-05$ & $3.6 \mathrm{E}-\odot 5$ \\
\hline SW & $5.8 \mathrm{E}-05$ & $5.6 \mathrm{E}-\odot 5$ & 4. ०E-๑5 & $3.8 \mathrm{E}-05$ & $3.5 E-\odot 5$ & $3.4 \mathrm{E}-05$ & $3.3 E-05$ \\
\hline SSW & $2.1 \mathrm{E}-05$ & 2. $\odot E-\odot 5$ & $1.5 \mathrm{E}-05$ & 1. $4 \mathrm{E}-05$ & 1. $3 E-05$ & 1. $3 E-05$ & 1. $3 E-05$ \\
\hline $\mathrm{S}$ & $3.1 \mathrm{E}-05$ & $3 . \odot E-\odot 5$ & $2.2 \mathrm{E}-\odot 5$ & $2.1 \mathrm{E}-05$ & 1. $9 \mathrm{E}-\odot 5$ & 1. $9 \mathrm{E}-05$ & 1. $8 \mathrm{E}-05$ \\
\hline SSE & $3.6 \mathrm{E}-05$ & $3.4 \mathrm{E}-05$ & $2.5 E-\odot 5$ & $2.4 \mathrm{E}-05$ & $2.2 \mathrm{E}-\odot 5$ & $2.1 \mathrm{E}-05$ & $2.1 \mathrm{E}-05$ \\
\hline SE & 1. $3 E-\odot 4$ & 1. 3E- $\odot 4$ & $9.6 \mathrm{E}-05$ & $9.2 \mathrm{E}-05$ & $8.5 E-\odot 5$ & $8.1 \mathrm{E}-05$ & 8. $0 \mathrm{E}-05$ \\
\hline ESE & $2.2 \mathrm{E}-\odot 4$ & $2.1 \mathrm{E}-\odot 4$ & 1. $7 \mathrm{E}-\odot 4$ & $1.6 E-\odot 4$ & $1.5 E-\odot 4$ & 1. $4 \mathrm{E}-\odot 4$ & 1. $4 \mathrm{E}-\odot 4$ \\
\hline E & $5.6 \mathrm{E}-05$ & $5.4 \mathrm{E}-05$ & $4.3 E-05$ & $4.2 E-05$ & $3.8 E-05$ & $3.7 \mathrm{E}-05$ & $3.6 \mathrm{E}-05$ \\
\hline ENE & $3.1 \mathrm{E}-05$ & $3 . \odot E-\odot 5$ & $2.4 \mathrm{E}-05$ & $2.3 E-05$ & $2.1 \mathrm{E}-\odot 5$ & $2 . \odot E-\odot 5$ & 2. $.0 E-05$ \\
\hline NE & $4.6 E-\odot 5$ & $4.5 E-\odot 5$ & $3.6 \mathrm{E}-05$ & $3.5 E-05$ & $3.2 \mathrm{E}-\odot 5$ & $3.1 \mathrm{E}-05$ & $3.1 \mathrm{E}-05$ \\
\hline NNE & $6.7 E-\odot 5$ & $6.5 E-\odot 5$ & $5.4 \mathrm{E}-\odot 5$ & $5.2 \mathrm{E}-\odot 5$ & $4.8 E-05$ & $4.6 \mathrm{E}-\odot 5$ & $4.5 E-05$ \\
\hline
\end{tabular}


Nov 27, 2006 05:40 pm

SUMMARY

Page 6

INDIVIDUAL EFFECTIVE DOSE EQUIVALENT RATE (mrem/y) (All Radionuclides and Pathways)

\begin{tabular}{|c|c|c|c|c|c|c|}
\hline \multirow[b]{2}{*}{ Direction } & \multicolumn{6}{|c|}{ Distance $(\mathrm{m})$} \\
\hline & 73520 & 75750 & 76380 & 78440 & 79130 & 79450 \\
\hline $\mathrm{N}$ & $4.2 E-05$ & 4. $0 \mathrm{E}-05$ & 4. . -05 & $3.8 E-05$ & $3.8 E-05$ & $3.7 E-05$ \\
\hline NNW & $2.1 \mathrm{E}-05$ & 2. $\odot E-\odot 5$ & $2 . \odot E-\odot 5$ & 1. $9 \mathrm{E}-05$ & 1. $9 \mathrm{E}-\odot 5$ & $1.9 \mathrm{E}-05$ \\
\hline NW & $1.6 \mathrm{E}-\odot 5$ & $1.5 \mathrm{E}-\odot 5$ & $1.5 E-\odot 5$ & $1.5 E-\odot 5$ & $1.5 E-\odot 5$ & 1. $4 \mathrm{E}-05$ \\
\hline WNW & 1.7E- -5 & $1.6 \mathrm{E}-05$ & $1.6 \mathrm{E}-05$ & $1.6 \mathrm{E}-05$ & $1.5 \mathrm{E}-05$ & $1.5 \mathrm{E}-05$ \\
\hline W & $2.9 \mathrm{E}-\odot 5$ & $2.8 \mathrm{E}-\odot 5$ & $2.7 \mathrm{E}-05$ & $2.6 E-\odot 5$ & $2.6 \mathrm{E}-\odot 5$ & $2.6 \mathrm{E}-05$ \\
\hline WSW & $3.4 \mathrm{E}-\odot 5$ & $3.2 \mathrm{E}-\odot 5$ & $3.2 \mathrm{E}-05$ & $3.1 \mathrm{E}-05$ & $3 . \odot E-\odot 5$ & $3.0 \mathrm{E}-05$ \\
\hline SW & $3.1 \mathrm{E}-05$ & $3.0 \mathrm{E}-05$ & $2.9 \mathrm{E}-05$ & $2.8 \mathrm{E}-05$ & $2.8 \mathrm{E}-05$ & $2.7 \mathrm{E}-05$ \\
\hline SSW & 1. $2 \mathrm{E}-\odot 5$ & $1.1 \mathrm{E}-\odot 5$ & 1. 1E- 05 & $1.1 \mathrm{E}-05$ & $1.1 \mathrm{E}-05$ & 1.1E- 05 \\
\hline $\mathrm{S}$ & 1.7E-05 & $1.6 \mathrm{E}-05$ & $1.6 \mathrm{E}-05$ & $1.5 \mathrm{E}-05$ & $1.5 E-05$ & $1.5 \mathrm{E}-05$ \\
\hline SSE & $2.0 \mathrm{E}-05$ & 1. $9 \mathrm{E}-05$ & 1. $9 \mathrm{E}-05$ & $1.8 \mathrm{E}-05$ & $1.8 \mathrm{E}-05$ & $1.8 \mathrm{E}-05$ \\
\hline SE & $7.5 E-\odot 5$ & $7.2 \mathrm{E}-\odot 5$ & $7.1 \mathrm{E}-05$ & $6.8 \mathrm{E}-\odot 5$ & $6.7 \mathrm{E}-\odot 5$ & $6.6 \mathrm{E}-\odot 5$ \\
\hline ESE & $1.3 E-\odot 4$ & $1.3 E-\odot 4$ & $1.3 E-\odot 4$ & $1.2 \mathrm{E}-\odot 4$ & $1.2 \mathrm{E}-\odot 4$ & 1. $2 \mathrm{E}-04$ \\
\hline E & $3.4 \mathrm{E}-05$ & $3.3 E-05$ & $3.2 \mathrm{E}-05$ & $3.1 \mathrm{E}-05$ & $3.1 \mathrm{E}-05$ & $3.0 \mathrm{E}-05$ \\
\hline ENE & 1. $9 \mathrm{E}-\odot 5$ & 1. $8 \mathrm{E}-\odot 5$ & 1. $8 \mathrm{E}-05$ & 1. 7E- $\odot 5$ & 1. 7E- $\odot 5$ & 1. $7 \mathrm{E}-05$ \\
\hline NE & $2.9 E-05$ & $2.7 \mathrm{E}-05$ & $2.7 \mathrm{E}-05$ & $2.6 \mathrm{E}-05$ & $2.6 E-05$ & $2.6 \mathrm{E}-05$ \\
\hline NNE & $4.2 \mathrm{E}-05$ & $4.1 E-\odot 5$ & 4. $\odot E-\odot 5$ & $3.9 \mathrm{E}-05$ & $3.8 \mathrm{E}-05$ & $3.8 \mathrm{E}-05$ \\
\hline
\end{tabular}


Nov 27, $2006 \quad 05: 40$ pm

SUMMARY

Page 7

INDIVIDUAL LIFETIME RISK (deaths)

(All Radionuclides and Pathways)

\begin{tabular}{|c|c|c|c|c|c|c|c|}
\hline \multirow[b]{2}{*}{ Direction } & \multicolumn{6}{|c|}{ Distance (m) } & \multirow[b]{2}{*}{54360} \\
\hline & 22600 & 46710 & 48940 & 49330 & 51120 & 53270 & \\
\hline $\mathrm{N}$ & $1.1 \mathrm{E}-10$ & $3.5 \mathrm{E}-11$ & $3.3 \mathrm{E}-11$ & 3. $2 \mathrm{E}-11$ & 3. $0 \mathrm{E}-11$ & $2.8 \mathrm{E}-11$ & $2.7 \mathrm{E}-11$ \\
\hline NNW & $5.5 \mathrm{E}-11$ & 1. $6 \mathrm{E}-11$ & 1. $5 \mathrm{E}-11$ & 1. $5 \mathrm{E}-11$ & 1. $4 \mathrm{E}-11$ & 1. $3 E-11$ & 1. $3 \mathrm{E}-11$ \\
\hline NW & $4.2 \mathrm{E}-11$ & 1. $3 E-11$ & 1. $2 \mathrm{E}-11$ & 1. $2 \mathrm{E}-11$ & 1.1E-11 & 1. $1 \mathrm{E}-11$ & 1. $0 \mathrm{E}-11$ \\
\hline WNW & $4.3 E-11$ & $1.4 \mathrm{E}-11$ & 1. $3 \mathrm{E}-11$ & 1. $3 \mathrm{E}-11$ & 1. $2 \mathrm{E}-11$ & $1.1 \mathrm{E}-11$ & $1.1 \mathrm{E}-11$ \\
\hline W & $9.3 \mathrm{E}-11$ & $2.8 \mathrm{E}-11$ & $2.6 \mathrm{E}-11$ & $2.5 \mathrm{E}-11$ & $2.4 \mathrm{E}-11$ & $2.2 \mathrm{E}-11$ & $2.1 \mathrm{E}-11$ \\
\hline WSW & $1.1 \mathrm{E}-10$ & $3.2 \mathrm{E}-11$ & 3. .E-11 & $2.9 \mathrm{E}-11$ & $2.8 \mathrm{E}-11$ & $2.6 \mathrm{E}-11$ & $2.5 \mathrm{E}-11$ \\
\hline SW & $1.1 \mathrm{E}-10$ & $3.2 \mathrm{E}-11$ & $2.9 \mathrm{E}-11$ & $2.9 \mathrm{E}-11$ & $2.7 \mathrm{E}-11$ & $2.5 \mathrm{E}-11$ & $2.4 \mathrm{E}-11$ \\
\hline SSW & 4. $3 E-11$ & 1. $2 \mathrm{E}-11$ & 1. $1 \mathrm{E}-11$ & 1. $1 \mathrm{E}-11$ & 1. $0 \mathrm{E}-11$ & $9.3 \mathrm{E}-12$ & $8.9 E-12$ \\
\hline $\mathrm{S}$ & $6.0 \mathrm{E}-11$ & $1.7 \mathrm{E}-11$ & $1.6 \mathrm{E}-11$ & 1. $5 \mathrm{E}-11$ & 1. $4 \mathrm{E}-11$ & 1. $3 \mathrm{E}-11$ & 1. $3 E-11$ \\
\hline SSE & $7.5 \mathrm{E}-11$ & $1.9 \mathrm{E}-11$ & $1.8 \mathrm{E}-11$ & 1.7E-11 & 1. $6 \mathrm{E}-11$ & $1.5 \mathrm{E}-11$ & 1. $5 \mathrm{E}-11$ \\
\hline SE & $2.4 \mathrm{E}-10$ & $6.8 \mathrm{E}-11$ & $6.3 \mathrm{E}-11$ & 6. $3 \mathrm{E}-11$ & $5.9 \mathrm{E}-11$ & $5.5 \mathrm{E}-11$ & $5.3 E-11$ \\
\hline ESE & $3.4 \mathrm{E}-10$ & $1.1 \mathrm{E}-10$ & 1. $0 \mathrm{E}-10$ & 1. $0 \mathrm{E}-10$ & $9.5 \mathrm{E}-11$ & $8.9 \mathrm{E}-11$ & $8 \cdot 6 \mathrm{E}-11$ \\
\hline$E$ & $9.1 \mathrm{E}-11$ & $2.8 \mathrm{E}-11$ & $2.6 \mathrm{E}-11$ & $2.6 \mathrm{E}-11$ & $2.5 \mathrm{E}-11$ & $2.3 \mathrm{E}-11$ & $2.2 \mathrm{E}-11$ \\
\hline ENE & $5.3 \mathrm{E}-11$ & $1.6 \mathrm{E}-11$ & 1. $5 \mathrm{E}-11$ & 1. $4 \mathrm{E}-11$ & 1. $4 \mathrm{E}-11$ & 1. $3 E-11$ & 1. $2 \mathrm{E}-11$ \\
\hline $\mathrm{NE}$ & $7.4 \mathrm{E}-11$ & $2.3 E-11$ & $2.1 \mathrm{E}-11$ & $2.1 \mathrm{E}-11$ & $2.0 \mathrm{E}-11$ & 1. $9 \mathrm{E}-11$ & $1.8 \mathrm{E}-11$ \\
\hline NNE & $1.0 \mathrm{E}-10$ & 3. 3E-11 & $3.1 \mathrm{E}-11$ & $3.1 \mathrm{E}-11$ & $2.9 \mathrm{E}-11$ & $2.7 \mathrm{E}-11$ & $2.6 \mathrm{E}-11$ \\
\hline
\end{tabular}

Distance $(\mathrm{m})$

\begin{tabular}{|c|c|c|c|c|c|c|c|}
\hline Direction & 58780 & 59110 & 61710 & $6360 \odot$ & 67530 & 69590 & 70340 \\
\hline $\mathrm{N}$ & 2. $2 \mathrm{E}-11$ & 2. $2 \mathrm{E}-11$ & $1.8 \mathrm{E}-11$ & $1.7 \mathrm{E}-11$ & $1.6 \mathrm{E}-11$ & $1.5 \mathrm{E}-11$ & $1.5 \mathrm{E}-11$ \\
\hline NNW & $1.1 \mathrm{E}-11$ & 1. $0 \mathrm{E}-11$ & $8.5 \mathrm{E}-12$ & $8.2 \mathrm{E}-12$ & $7.6 \mathrm{E}-12$ & 7. $3 E-12$ & $7.2 \mathrm{E}-12$ \\
\hline NW & $8.4 \mathrm{E}-12$ & $8.2 \mathrm{E}-12$ & 6. $7 \mathrm{E}-12$ & $6.4 \mathrm{E}-12$ & 6. $0 \mathrm{E}-12$ & $5.8 \mathrm{E}-12$ & $5.7 \mathrm{E}-12$ \\
\hline WNW & $9.0 \mathrm{E}-12$ & $8.8 \mathrm{E}-12$ & $7.1 \mathrm{E}-12$ & $6.8 \mathrm{E}-12$ & $6.3 \mathrm{E}-12$ & $6.1 \mathrm{E}-12$ & 6. $0 \mathrm{E}-12$ \\
\hline W & $1.7 \mathrm{E}-11$ & $1.6 \mathrm{E}-11$ & 1. $2 \mathrm{E}-11$ & 1. $2 \mathrm{E}-11$ & 1.1E-11 & 1. $0 \mathrm{E}-11$ & 1. $0 \mathrm{E}-11$ \\
\hline WSW & $2.0 \mathrm{E}-11$ & 1. $9 \mathrm{E}-11$ & 1. $4 \mathrm{E}-11$ & 1. $4 \mathrm{E}-11$ & 1. $3 \mathrm{E}-11$ & 1. $2 \mathrm{E}-11$ & 1. $2 \mathrm{E}-11$ \\
\hline SW & $1.9 \mathrm{E}-11$ & 1. $8 \mathrm{E}-11$ & 1. $3 \mathrm{E}-11$ & 1. $3 \mathrm{E}-11$ & 1. $2 \mathrm{E}-11$ & 1. $1 \mathrm{E}-11$ & $1.1 \mathrm{E}-11$ \\
\hline SSW & $7.0 \mathrm{E}-12$ & $6.8 \mathrm{E}-12$ & 5. $0 \mathrm{E}-12$ & $4.8 \mathrm{E}-12$ & $4.5 \mathrm{E}-12$ & 4. $3 \mathrm{E}-12$ & $4.2 \mathrm{E}-12$ \\
\hline$S$ & 1. $0 \mathrm{E}-11$ & $9.8 \mathrm{E}-12$ & 7. 3E-12 & 7. $0 \mathrm{E}-12$ & $6.4 \mathrm{E}-12$ & $6.2 \mathrm{E}-12$ & $6.1 \mathrm{E}-12$ \\
\hline SSE & 1. $2 \mathrm{E}-11$ & $1.1 \mathrm{E}-11$ & 8. $3 \mathrm{E}-12$ & 8. $0 \mathrm{E}-12$ & $7.4 \mathrm{E}-12$ & $7.1 \mathrm{E}-12$ & 7. $0 \mathrm{E}-12$ \\
\hline SE & $4.2 \mathrm{E}-11$ & $4.1 \mathrm{E}-11$ & $3.1 \mathrm{E}-11$ & 3. $0 \mathrm{E}-11$ & $2.8 \mathrm{E}-11$ & $2.7 \mathrm{E}-11$ & $2.6 \mathrm{E}-11$ \\
\hline ESE & 7. $0 \mathrm{E}-11$ & $6.8 \mathrm{E}-11$ & $5.5 \mathrm{E}-11$ & $5.3 \mathrm{E}-11$ & $4.9 \mathrm{E}-11$ & 4. $7 \mathrm{E}-11$ & $4.6 \mathrm{E}-11$ \\
\hline E & $1.8 \mathrm{E}-11$ & $1.8 \mathrm{E}-11$ & 1. $4 \mathrm{E}-11$ & 1. $4 \mathrm{E}-11$ & 1. $3 \mathrm{E}-11$ & 1. $2 \mathrm{E}-11$ & 1. $2 \mathrm{E}-11$ \\
\hline ENE & 1. $0 \mathrm{E}-11$ & $9.8 \mathrm{E}-12$ & 7. $9 \mathrm{E}-12$ & $7.6 \mathrm{E}-12$ & 7. $0 \mathrm{E}-12$ & 6. $7 \mathrm{E}-12$ & $6.6 \mathrm{E}-12$ \\
\hline NE & $1.5 \mathrm{E}-11$ & $1.5 \mathrm{E}-11$ & 1. $2 \mathrm{E}-11$ & $1.1 \mathrm{E}-11$ & $1.1 \mathrm{E}-11$ & 1. $0 \mathrm{E}-11$ & 1. $0 \mathrm{E}-11$ \\
\hline NNE & $2.2 \mathrm{E}-11$ & $2.1 \mathrm{E}-11$ & $1.8 \mathrm{E}-11$ & $1.7 \mathrm{E}-11$ & $1.6 \mathrm{E}-11$ & 1. $5 \mathrm{E}-11$ & $1.5 \mathrm{E}-11$ \\
\hline
\end{tabular}


Nov 27, 2006 05:40 pm

SUMMARY

Page 8

INDIVIDUAL LIFETIME RISK (deaths)

(All Radionuclides and Pathways)

\begin{tabular}{|c|c|c|c|c|c|c|}
\hline \multirow[b]{2}{*}{ Direction } & \multicolumn{6}{|c|}{ Distance $(\mathrm{m})$} \\
\hline & 73520 & 75750 & 76380 & 78440 & 79130 & 79450 \\
\hline $\mathrm{N}$ & 1. $4 \mathrm{E}-11$ & 1. $3 \mathrm{E}-11$ & 1. $3 \mathrm{E}-11$ & 1. $3 \mathrm{E}-11$ & 1. $2 \mathrm{E}-11$ & 1. $2 \mathrm{E}-11$ \\
\hline NNW & $6.8 \mathrm{E}-12$ & $6.6 \mathrm{E}-12$ & $6.5 \mathrm{E}-12$ & $6.3 E-12$ & $6.2 \mathrm{E}-12$ & $6.2 \mathrm{E}-12$ \\
\hline NW & $5.4 \mathrm{E}-12$ & 5. $2 \mathrm{E}-12$ & $5.1 \mathrm{E}-12$ & $4.9 \mathrm{E}-12$ & $4.9 \mathrm{E}-12$ & 4. $8 \mathrm{E}-12$ \\
\hline WNW & $5.7 \mathrm{E}-12$ & $5.4 \mathrm{E}-12$ & $5.4 \mathrm{E}-12$ & $5.2 \mathrm{E}-12$ & $5.1 \mathrm{E}-12$ & $5.1 \mathrm{E}-12$ \\
\hline W & $9.5 \mathrm{E}-12$ & $9.1 \mathrm{E}-12$ & $9.0 \mathrm{E}-12$ & $8.6 \mathrm{E}-12$ & $8.5 \mathrm{E}-12$ & $8.5 E-12$ \\
\hline WSW & $1.1 \mathrm{E}-11$ & $1.1 \mathrm{E}-11$ & 1. $0 \mathrm{E}-11$ & 1. $0 \mathrm{E}-11$ & $9.9 \mathrm{E}-12$ & $9.8 \mathrm{E}-12$ \\
\hline SW & 1. $0 \mathrm{E}-11$ & $9.8 \mathrm{E}-12$ & $9.7 \mathrm{E}-12$ & $9.2 \mathrm{E}-12$ & $9.1 \mathrm{E}-12$ & 9. $\mathrm{OE}-12$ \\
\hline SSW & 4. $\mathrm{OE}-12$ & $3.8 \mathrm{E}-12$ & $3.8 \mathrm{E}-12$ & $3.6 \mathrm{E}-12$ & $3.6 \mathrm{E}-12$ & $3.6 \mathrm{E}-12$ \\
\hline $\mathrm{S}$ & $5.7 \mathrm{E}-12$ & $5.4 \mathrm{E}-12$ & $5.4 \mathrm{E}-12$ & $5.2 \mathrm{E}-12$ & $5.1 \mathrm{E}-12$ & $5.0 \mathrm{E}-12$ \\
\hline SSE & $6.6 \mathrm{E}-12$ & 6. $3 \mathrm{E}-12$ & $6.2 \mathrm{E}-12$ & 6. $\odot \mathrm{E}-12$ & $5.9 \mathrm{E}-12$ & $5.9 \mathrm{E}-12$ \\
\hline SE & $2.4 \mathrm{E}-11$ & $2.3 \mathrm{E}-11$ & $2.3 \mathrm{E}-11$ & $2.2 \mathrm{E}-11$ & $2.2 \mathrm{E}-11$ & $2.2 \mathrm{E}-11$ \\
\hline ESE & 4. $3 \mathrm{E}-11$ & $4.1 \mathrm{E}-11$ & $4.1 \mathrm{E}-11$ & $3.9 \mathrm{E}-11$ & $3.9 \mathrm{E}-11$ & $3.8 \mathrm{E}-11$ \\
\hline$E$ & 1.1E-11 & 1. $1 \mathrm{E}-11$ & 1. $1 \mathrm{E}-11$ & 1. $0 \mathrm{E}-11$ & 1. $0 \mathrm{E}-11$ & 1. $0 \mathrm{E}-11$ \\
\hline ENE & $6.2 \mathrm{E}-12$ & $6.0 \mathrm{E}-12$ & $5.9 \mathrm{E}-12$ & $5.7 \mathrm{E}-12$ & $5.6 \mathrm{E}-12$ & $5.6 \mathrm{E}-12$ \\
\hline NE & $9.5 \mathrm{E}-12$ & $9.1 \mathrm{E}-12$ & $9.0 \mathrm{E}-12$ & $8.6 \mathrm{E}-12$ & $8.5 \mathrm{E}-12$ & $8.5 \mathrm{E}-12$ \\
\hline NNE & 1. $4 \mathrm{E}-11$ & 1. $3 \mathrm{E}-11$ & 1. $3 \mathrm{E}-11$ & 1. $3 E-11$ & 1. $2 \mathrm{E}-11$ & 1. $2 \mathrm{E}-11$ \\
\hline
\end{tabular}




\author{
C A P 88 - P C \\ Version 3.0 \\ Clean Air Act Assessment Package - 1988 \\ WE A T H E R D A T A \\ Non-Radon Individual Assessment \\ Nov 27, 2006 05:40 pm

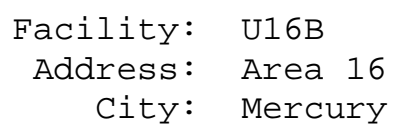

Comments: Dose from the proposed Divine Strake experiment: 2005 average wind conditions used. Source used was maximum upper confidence interval reported in DOE/NV-1177 and total PM10.

Dataset Name: DS SNJV Max UCI

Dataset Date: 10/26/2006 8:58:00 AM

Wind File: C: \Program Files\CAP88-PC30\WindLib\Divine

Stake \05MEDA21. WND 
Appendix A

Nov 27, 2006 05:40 pm

WEATHER

Page 1

HARMONIC AVERAGE WIND SPEEDS (WIND TOWARDS)

\begin{tabular}{|c|c|c|c|c|c|c|c|c|}
\hline \multirow[b]{2}{*}{ Dir } & \multicolumn{7}{|c|}{ Pasquill Stability Class } & \multirow[b]{2}{*}{$\begin{array}{l}\text { Wind } \\
\text { Freq }\end{array}$} \\
\hline & $A$ & B & C & $D$ & $E$ & $\mathrm{~F}$ & G & \\
\hline $\mathrm{N}$ & 0.954 & 1.436 & 1.909 & 3.479 & 3.841 & 1.260 & 0.772 & ๑. 096 \\
\hline NNW & 1.007 & 1.123 & 1.652 & 4.429 & 3.911 & 1.870 & 0.772 & 0.038 \\
\hline NW & 1.007 & 0.906 & 2.223 & 2.500 & 3.969 & 1.665 & 0.772 & 0.023 \\
\hline WNW & 0.772 & 0.953 & 1.017 & 1.876 & 3.802 & 2.175 & 0.772 & 0.020 \\
\hline W & 0.846 & 0.849 & 1.067 & 1.474 & 3.430 & 1.734 & 0.772 & 0.033 \\
\hline WSW & 0.772 & 0.841 & 1.092 & 1.696 & 3.332 & 1.708 & 0.772 & ๑. .041 \\
\hline SW & 0.772 & ๑. 897 & 0.994 & 2.624 & 3.611 & 1.327 & 0.772 & 0.045 \\
\hline SSW & ๑. 897 & 0.953 & 1.044 & 2.502 & 3.701 & 1.098 & 0.772 & ๑. 022 \\
\hline S & 0.772 & 0.915 & 1.175 & 1.559 & 3.938 & 1.251 & 0.772 & ๑. .029 \\
\hline SSE & 0.807 & 1.136 & 2.226 & 2.268 & 3.430 & 1.557 & 0.772 & $\odot .042$ \\
\hline SE & 0.924 & 1.184 & 2.061 & 2.824 & 3.794 & 1.622 & 0.772 & $\odot .127$ \\
\hline ESE & 1.007 & 1.326 & 2.102 & 2.818 & 3.795 & 2.023 & $\odot .772$ & $\odot .189$ \\
\hline$E$ & 1.330 & 1.332 & 1.744 & 3.228 & 3.341 & 2.026 & 0.772 & ๑. 059 \\
\hline ENE & 1.046 & 1.710 & 3.060 & 2.663 & 3.976 & 1.611 & 0.772 & $\odot .049$ \\
\hline NE & 1.781 & 2.116 & 3.395 & 2.969 & 3.902 & 1.625 & 0.772 & ๑. 080 \\
\hline NNE & 1.089 & 1.369 & 2.902 & 3.997 & 4.006 & 1.573 & 0.772 & 0.108 \\
\hline
\end{tabular}

ARITHMETIC AVERAGE WIND SPEEDS (WIND TOWARDS)

Pasquill Stability Class

\begin{tabular}{|c|c|c|c|c|c|c|c|}
\hline Dir & $A$ & B & C & D & $E$ & $F$ & G \\
\hline $\mathrm{N}$ & 1.263 & 2.349 & 3.930 & 6.572 & 4.017 & 1.769 & 0.772 \\
\hline NNW & 1.372 & 1.905 & 3.155 & 6.254 & 4.069 & 2.283 & 0.772 \\
\hline NW & 1.372 & 1.252 & 3.087 & 4.444 & 4.111 & 2.152 & 0.772 \\
\hline WNW & 0.772 & 1.447 & 1.929 & 3.419 & 3.987 & 2.431 & 0.772 \\
\hline W & 0.997 & 1.172 & 1.754 & 2.944 & 3.665 & 2.199 & 0.772 \\
\hline WSW & 0.772 & 1.132 & 1.897 & 4.032 & 3.570 & 2.182 & 0.772 \\
\hline SW & 0.772 & 1.132 & 1.629 & 4.852 & 3.830 & 1.849 & 0.772 \\
\hline SSW & 1.132 & 1.447 & 1.711 & 4.493 & 3.906 & 1.536 & 0.772 \\
\hline S & 0.772 & 1.221 & 2.272 & 3.330 & 4.088 & 1.757 & $\odot .772$ \\
\hline SSE & 0.884 & 1.630 & 3.292 & 4.093 & 3.665 & 2.069 & 0.772 \\
\hline SE & 1.195 & 1.839 & 3.308 & 4.613 & 3.980 & 2.120 & 0.772 \\
\hline ESE & 1.372 & 2.129 & 3.047 & 4.305 & 3.981 & 2.363 & 0.772 \\
\hline$E$ & 1.852 & 2.001 & 3.138 & 4.818 & 3.579 & 2.364 & 0.772 \\
\hline ENE & 1.447 & 2.741 & 4.929 & 4.926 & 4.116 & 2.111 & 0.772 \\
\hline NE & 2.229 & 3.090 & 4.580 & 5.348 & 4.063 & 2.123 & $\odot .772$ \\
\hline NNE & 1.522 & 2.307 & 4.289 & 5.784 & 4.137 & 2.082 & 0.772 \\
\hline
\end{tabular}


FREQUENCIES OF STABILITY CLASSES (WIND TOWARDS)

\begin{tabular}{|c|c|c|c|c|c|c|c|}
\hline \multirow[b]{2}{*}{ Dir } & \multicolumn{7}{|c|}{ Pasquill Stability Class } \\
\hline & A & B & C & D & E & $\mathrm{F}$ & G \\
\hline $\mathrm{N}$ & 0.0152 & 0.0900 & 0.0899 & 0.5056 & 0.1330 & 0.1026 & 0.0637 \\
\hline NNW & ๑. . 0104 & 0.0940 & 0.0522 & 0.3135 & 0.2683 & 0.1082 & 0.1534 \\
\hline NW & $\odot .0170$ & ๑. 0852 & ๑. 0397 & $\odot .2271$ & $\odot .3127$ & $\odot .1703$ & 0.1480 \\
\hline WNW & $\odot .0204$ & $\odot .0544$ & $\odot .0680$ & $\odot .1632$ & $\odot .1904$ & 0.3473 & 0.1564 \\
\hline W & 0.0325 & 0.0365 & 0.0447 & $\odot .0731$ & 0.1137 & 0.4719 & 0.2277 \\
\hline WSW & $\odot .0292$ & 0.0324 & $\odot .0259$ & 0.1394 & $\odot .1527$ & $\odot .3899$ & 0.2305 \\
\hline SW & $\odot .0 \odot 29$ & 0.0439 & 0.0615 & 0.1584 & $\odot .1847$ & $\odot .3285$ & 0.2201 \\
\hline SSW & 0.0303 & 0.0969 & 0.1212 & 0.1938 & 0.1636 & $\odot .1999$ & 0.1943 \\
\hline S & $\odot .0325$ & 0.1490 & $\odot .0557$ & $\odot .2601$ & $\odot .0882$ & $\odot .2469$ & 0.1676 \\
\hline SSE & 0.0511 & 0.1407 & 0.0958 & 0.2138 & 0.0894 & 0.1598 & 0.2494 \\
\hline SE & 0.0178 & 0.0903 & 0.0724 & 0.2602 & 0.1638 & 0.2214 & 0.1742 \\
\hline ESE & ๑. 0105 & $\odot .0999$ & ๑. 0584 & $\odot .2413$ & $\odot .2104$ & $\odot .26 \odot 4$ & 0.1190 \\
\hline$E$ & 0.0225 & 0.1356 & 0.1127 & 0.3025 & 0.0971 & 0.2145 & 0.1151 \\
\hline ENE & 0.0215 & 0.2287 & 0.2580 & 0.2229 & 0.0753 & 0.1049 & 0.0888 \\
\hline $\mathrm{NE}$ & $\odot .0348$ & 0.2775 & 0.1928 & $\odot .2490$ & $\odot .0963$ & $\odot .0864$ & 0.0632 \\
\hline NNE & 0.0296 & 0.1507 & 0.1530 & 0.4284 & 0.1037 & 0.0950 & 0.0395 \\
\hline TOTAL & 0.0214 & 0.1201 & 0.0980 & 0.2783 & 0.1510 & 0.2013 & 0.1298 \\
\hline
\end{tabular}

ADDITIONAL WEATHER INFORMATION

Average Air Temperature: 13.2 degrees $\mathrm{C}$ $286.36 \mathrm{~K}$

Precipitation: $\quad 3.0 \mathrm{~cm} / \mathrm{y}$ Humidity: $\quad 3.0 \mathrm{~g} / \mathrm{cu} \mathrm{m}$ Lid Height: 3000 meters

Surface Roughness Length: 0.010 meters Height of Wind Measurements: 10.0 meters Average Wind Speed: $3.230 \mathrm{~m} / \mathrm{s}$

Vertical Temperature Gradients:

$\begin{array}{lll}\text { STABILITY E } & 0.073 \mathrm{k} / \mathrm{m} \\ \text { STABILITY F } & 0.109 \mathrm{k} / \mathrm{m} \\ \text { STABILITY G } & 0.146 \mathrm{k} / \mathrm{m}\end{array}$




\author{
C A P 88 - P C \\ Version $3 . \odot$ \\ Clean Air Act Assessment Package - 1988 \\ GE NERAL DA T A \\ Non-Radon Individual Assessment \\ Nov 27, $2006 \quad 05: 40 \mathrm{pm}$
}

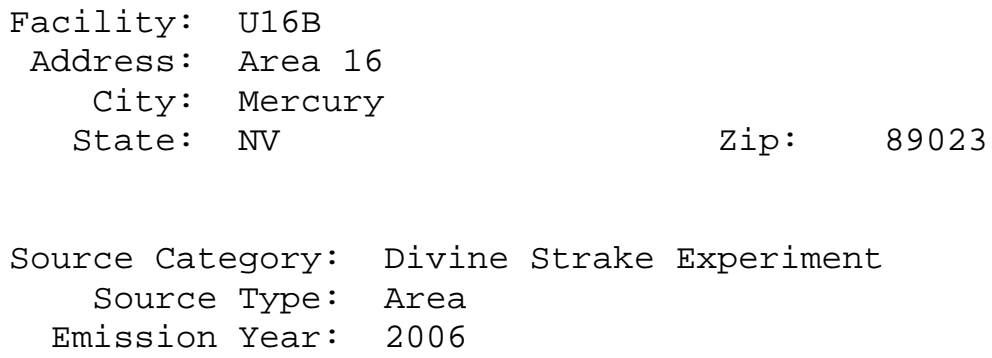

Comments: Dose from the proposed Divine Strake experiment: 2005 average wind conditions used. Source used was maximum upper confidence interval reported in DOE/NV-1177 and total PM10.

Dataset Name: DS SNJV Max UCI

Dataset Date: 10/26/2006 8:58:00 AM

Wind File: C: \Program Files\CAP88-PC30\WindLib\Divine 


\section{VALUES FOR RADIONUCLIDE-DEPENDENT PARAMETERS}

\begin{tabular}{|c|c|c|c|c|}
\hline Nuclide & $\begin{array}{l}\text { Clearance } \\
\text { Type }\end{array}$ & $\begin{array}{l}\text { Particle } \\
\text { Size } \\
\text { (microns) }\end{array}$ & $\begin{array}{l}\text { Scavenging } \\
\text { Coefficient } \\
\text { (per second) }\end{array}$ & $\begin{array}{c}\text { Dry } \\
\text { Deposition } \\
\text { Velocity } \\
(\mathrm{m} / \mathrm{s})\end{array}$ \\
\hline Cs - 137 & $\mathrm{~F}$ & 1 & $3.00 E-07$ & $1.80 E-03$ \\
\hline $\mathrm{Ba}-137 \mathrm{~m}$ & $M$ & 1 & $3.00 E-07$ & $1.80 E-03$ \\
\hline Am- 241 & $M$ & 1 & $3.00 E-07$ & $1.80 E-03$ \\
\hline$N p-237$ & $M$ & 1 & $3.00 \mathrm{E}-07$ & 1. 80E-०3 \\
\hline $\mathrm{Pa}-233$ & $M$ & 1 & $3.00 E-07$ & $1.80 E-03$ \\
\hline$U-233$ & $M$ & 1 & $3.00 E-07$ & $1.80 E-03$ \\
\hline Th - 229 & $\mathrm{~S}$ & 1 & $3.00 E-07$ & $1.80 E-03$ \\
\hline $\mathrm{Ra}-225$ & $M$ & 1 & $3.00 \mathrm{E}-07$ & $1.80 E-03$ \\
\hline$A c-225$ & $M$ & 1 & $3.00 E-07$ & $1.80 E-03$ \\
\hline Fr - 221 & $M$ & 1 & $3.00 E-\odot 7$ & 1. 80E- - 3 \\
\hline At -217 & $M$ & 1 & $3.00 \mathrm{E}-\odot 7$ & 1. 80E- - 3 \\
\hline $\mathrm{Bi}-213$ & $M$ & 1 & $3.00 \mathrm{E}-07$ & $1.80 E-03$ \\
\hline Po- 213 & $M$ & 1 & $3.00 E-07$ & $1.80 E-03$ \\
\hline$P b-209$ & $M$ & 1 & $3.0 \odot E-\odot 7$ & 1. 80E- - 3 \\
\hline Tl- 209 & $M$ & 1 & $3.00 \mathrm{E}-\odot 7$ & 1. 80E- - 3 \\
\hline Pu- 238 & $M$ & 1 & $3.00 \mathrm{E}-07$ & $1.80 E-03$ \\
\hline U-234 & $M$ & 1 & $3 . \odot \odot E-\odot 7$ & 1. 80E-०3 \\
\hline Th - 230 & $\mathrm{~S}$ & 1 & $3 . \odot \odot E-\odot 7$ & 1. 80E- - 3 \\
\hline $\mathrm{Ra}-226$ & $M$ & 1 & $3.00 E-\odot 7$ & 1. 80E- - 3 \\
\hline Rn - 222 & $\mathrm{G}$ & $\odot$ & $\odot . \odot \odot E+\odot \odot$ & $\odot . \odot \odot E+\odot \odot$ \\
\hline Po- 218 & $M$ & 1 & $3.00 \mathrm{E}-07$ & 1. 80E- - 3 \\
\hline $\mathrm{Pb}-214$ & $M$ & 1 & $3.00 \mathrm{E}-\odot 7$ & 1. 80E- - 3 \\
\hline $\mathrm{Bi}-214$ & $M$ & 1 & 3. ००E - ०7 & 1. 80E- - 33 \\
\hline Po- 214 & $M$ & 1 & 3. ००E - ०७ & $1.80 E-03$ \\
\hline $\mathrm{Pb}-210$ & M & 1 & 3. ००E - ๑7 & 1. 80E- - 3 \\
\hline $\mathrm{Bi}-210$ & $M$ & 1 & 3. ००E - ०7 & 1. 80E- - 3 \\
\hline Po- 210 & M & 1 & $3.0 \odot E-\odot 7$ & 1. 80E-०3 \\
\hline At - 218 & $M$ & 1 & 3. ००E - ०७ & 1. 80E- - 3 \\
\hline Sr-90 & $M$ & 1 & 3. ००E - ๑7 & 1. 8०E-०3 \\
\hline$Y-90$ & $M$ & 1 & 3. ००E - ๑7 & 1. 80E-०3 \\
\hline $\mathrm{K}-40$ & $M$ & 1 & 3. ००E - ०7 & 1. 80E-०3 \\
\hline Th- 232 & $\mathrm{~S}$ & 1 & $3.00 E-07$ & $1.80 E-03$ \\
\hline $\mathrm{Ra}-228$ & $M$ & 1 & $3.00 E-07$ & $1.80 E-03$ \\
\hline$A c-228$ & $M$ & 1 & 3. ००E - ०7 & 1. 80E-०3 \\
\hline Th - 228 & $\mathrm{~S}$ & 1 & $3.00 E-07$ & $1.80 E-03$ \\
\hline $\mathrm{Ra}-224$ & $M$ & 1 & $3.00 E-07$ & $1.80 E-03$ \\
\hline Rn - 220 & G & $\odot$ & $\odot .00 \mathrm{E}+0 \odot$ & $\odot . \odot \odot E+\odot \odot$ \\
\hline Po- 216 & $M$ & 1 & $3.00 E-07$ & $1.80 E-03$ \\
\hline $\mathrm{Pb}-212$ & $M$ & 1 & $3.00 E-07$ & $1.80 E-03$ \\
\hline $\mathrm{Bi}-212$ & $M$ & 1 & 3. ००E - ๑7 & 1. 80E-०3 \\
\hline Po- 212 & $M$ & 1 & $3.00 E-07$ & $1.80 E-03$ \\
\hline Tl- 208 & $M$ & 1 & 3. ००E - ๑7 & $1.80 E-03$ \\
\hline$U-238$ & $M$ & 1 & $3.00 E-07$ & $1.80 E-03$ \\
\hline Th - 234 & $\mathrm{~S}$ & 1 & $3.0 \odot E-\odot 7$ & 1. 80E-०3 \\
\hline
\end{tabular}


Appendix A

$\mathrm{Pa}-234 \mathrm{~m}$
$\mathrm{~Pa}-234$
$\mathrm{Al}-26$
$\mathrm{Be}-7$
$\mathrm{Cm}-243$
$\mathrm{Am}-243$
$\mathrm{~Np}-239$
$\mathrm{Pu}-239$
$\mathrm{U}-235$
$\mathrm{Th}-231$
$\mathrm{~Pa}-231$
$\mathrm{AC}-227$
$\mathrm{Th}-227$
$\mathrm{Ra}-223$
$\mathrm{Rn}-219$
$\mathrm{Po}-215$
$\mathrm{~Pb}-211$
$\mathrm{Bi}-211$
$\mathrm{Tl}-207$
$\mathrm{Po}-211$
$\mathrm{Fr}-223$
$\mathrm{Co}-58$
$\mathrm{Co}-60$
$\mathrm{CS}-134$
$\mathrm{Eu}-152$
$\mathrm{Gd}-152$
$\mathrm{Eu}-154$
$\mathrm{Eu}-155$
$\mathrm{Nb}-94$
$\mathrm{Sb}-125$
$\mathrm{Te}-125 \mathrm{~m}$
$\mathrm{H}-3$

$\begin{array}{ll}M & 1 \\ M & 1 \\ M & 1 \\ M & 1 \\ M & 1 \\ M & 1 \\ M & 1 \\ M & 1 \\ M & 1 \\ S & 1 \\ M & 1 \\ M & 1 \\ S & 1 \\ M & 1 \\ G & 0 \\ M & 1 \\ M & 1 \\ M & 1 \\ M & 1 \\ M & 1 \\ M & 1 \\ M & 1 \\ M & 1 \\ F & 1 \\ M & 1 \\ M & 1 \\ M & 1 \\ M & 1 \\ M & 1 \\ M & 1 \\ M & 0 \\ V & \end{array}$

\begin{tabular}{|c|c|}
\hline 3. $00 \mathrm{E}-07$ & $1.80 \mathrm{E}-03$ \\
\hline 3. $\odot \odot E-\odot 7$ & $1.80 \mathrm{E}-03$ \\
\hline 3. ००E - ๑7 & $1.80 \mathrm{E}-03$ \\
\hline 3. $\odot \odot E-\odot 7$ & $1.80 E-\odot 3$ \\
\hline 3. $\odot \odot E-\odot 7$ & $1.80 \mathrm{E}-03$ \\
\hline 3. $0 \odot \mathrm{E}-\odot 7$ & $1.80 \mathrm{E}-03$ \\
\hline $3 . \odot \odot E-\odot 7$ & $1.80 \mathrm{E}-03$ \\
\hline 3. ๑९E - ๑7 & $1.80 E-\odot 3$ \\
\hline 3. ००E- - 7 & $1.80 \mathrm{E}-03$ \\
\hline 3. $\odot \odot E-\odot 7$ & $1.80 \mathrm{E}-03$ \\
\hline 3. $\odot \odot E-\odot 7$ & $1.80 \mathrm{E}-03$ \\
\hline 3. $\odot \odot E-\odot 7$ & $1.80 \mathrm{E}-03$ \\
\hline 3. ००E - ๑7 & $1.80 \mathrm{E}-03$ \\
\hline $3 . \odot \odot E-\odot 7$ & $1.80 \mathrm{E}-\odot 3$ \\
\hline$\odot . \odot \odot E+\odot \odot$ & $\odot . \odot \odot E+\odot \odot$ \\
\hline $3.00 \mathrm{E}-07$ & $1.80 E-03$ \\
\hline $3.00 \mathrm{E}-\odot 7$ & 1. $80 \mathrm{E}-03$ \\
\hline 3. ००E-๑7 & $1.80 \mathrm{E}-03$ \\
\hline 3. $\odot \odot E-\odot 7$ & $1.80 \mathrm{E}-03$ \\
\hline 3. ००E - ๑7 & $1.80 \mathrm{E}-03$ \\
\hline 3. ๑९E - ๑7 & $1.80 E-03$ \\
\hline 3. $\odot \odot E-\odot 7$ & $1.80 \mathrm{E}-03$ \\
\hline 3. ००E-๑7 & $1.80 \mathrm{E}-03$ \\
\hline 3. $\odot \odot E-\odot 7$ & $1.80 \mathrm{E}-03$ \\
\hline $3.00 E-\odot 7$ & 1. $80 \mathrm{E}-03$ \\
\hline 3. $0 \odot \mathrm{E}-\odot 7$ & $1.80 \mathrm{E}-03$ \\
\hline 3. ००E-๑7 & $1.80 \mathrm{E}-\odot 3$ \\
\hline 3. $\odot \odot E-\odot 7$ & $1.80 \mathrm{E}-03$ \\
\hline 3. $0 \odot \mathrm{E}-\odot 7$ & $1.80 \mathrm{E}-03$ \\
\hline $3 . \odot \odot E-\odot 7$ & $1.80 \mathrm{E}-03$ \\
\hline 3. $\odot \odot E-\odot 7$ & 1. $80 \mathrm{E}-\odot 3$ \\
\hline$\odot . \odot \odot E+\odot \odot$ & $\odot . \odot \odot E+\odot \odot$ \\
\hline
\end{tabular}


VALUES FOR RADIONUCLIDE-DEPENDENT PARAMETERS

\begin{tabular}{|c|c|c|c|c|c|}
\hline \multirow[b]{2}{*}{ Nuclide } & \multicolumn{3}{|c|}{ DECAY CONSTANT (PER DAY) } & \multicolumn{2}{|c|}{ TRANSFER COEFFICIENT } \\
\hline & $\begin{array}{l}\text { Radio- } \\
\text { active (1) }\end{array}$ & Surface & Water & Milk (2) & Meat (3) \\
\hline Cs -137 & $6.32 \mathrm{E}-05$ & $5.48 \mathrm{E}-05$ & $\odot . \odot \odot E+\odot \odot$ & $1.00 \mathrm{E}-02$ & $5.00 \mathrm{E}-02$ \\
\hline $\mathrm{Ba}-137 \mathrm{~m}$ & $3.91 \mathrm{E}+02$ & $5.48 E-05$ & $\odot . \odot \odot E+\odot \odot$ & $5.00 \mathrm{E}-04$ & $2.00 \mathrm{E}-\odot 4$ \\
\hline$A m-241$ & $4.39 E-06$ & $5.48 E-05$ & $\odot . \odot \odot E+\odot \odot$ & $2.00 E-06$ & $5.00 E-05$ \\
\hline$N p-237$ & $8.87 \mathrm{E}-10$ & $5.48 \mathrm{E}-05$ & $\odot . \odot \odot E+\odot \odot$ & $1.00 \mathrm{E}-05$ & $1.00 \mathrm{E}-03$ \\
\hline$P a-233$ & $2.57 \mathrm{E}-02$ & $5.48 E-\odot 5$ & $\odot . \odot \odot E+\odot \odot$ & $5.00 \mathrm{E}-\odot 6$ & $5 . \odot \odot E-\odot 6$ \\
\hline$U-233$ & $1.20 \mathrm{E}-08$ & $5.48 \mathrm{E}-05$ & $\Theta .0 \odot E+\Theta \odot$ & $4.00 \mathrm{E}-04$ & 8. $00 \mathrm{E}-04$ \\
\hline Th-229 & $2.58 \mathrm{E}-07$ & $5.48 \mathrm{E}-05$ & $\odot . \odot \odot E+\odot \odot$ & $5.00 \mathrm{E}-\odot 6$ & $1.0 \odot E-\odot 4$ \\
\hline $\mathrm{Ra}-225$ & $4.68 \mathrm{E}-02$ & $5.48 E-05$ & $\odot . \odot \odot E+\odot \odot$ & $1.00 \mathrm{E}-03$ & $2 . \odot \odot E-\odot 3$ \\
\hline$A C-225$ & $6.93 E-02$ & $5.48 \mathrm{E}-05$ & $\odot . \odot \odot E+\odot \odot$ & $2.00 \mathrm{E}-06$ & $2.00 \mathrm{E}-05$ \\
\hline $\mathrm{Fr}-221$ & $2.08 \mathrm{E}+02$ & $5.48 \mathrm{E}-05$ & $\odot . \odot \odot E+\odot \odot$ & $8.00 \mathrm{E}-03$ & $3.00 \mathrm{E}-02$ \\
\hline At -217 & $1.85 E+\odot 6$ & $5.48 \mathrm{E}-05$ & $\odot . \odot \odot E+\odot \odot$ & $1.00 \mathrm{E}-\odot 2$ & 1. $\odot \odot E-\odot 2$ \\
\hline $\mathrm{Bi}-213$ & $2.19 \mathrm{E}+01$ & $5.48 \mathrm{E}-05$ & $\Theta .00 \mathrm{E}+0 \odot$ & $1.00 \mathrm{E}-03$ & $2.00 E-03$ \\
\hline Po- 213 & $1.43 \mathrm{E}+10$ & $5.48 \mathrm{E}-05$ & $\odot . \odot \odot E+\odot \odot$ & $4.00 E-04$ & $5.00 E-03$ \\
\hline$P b-209$ & $5.11 \mathrm{E}+\Theta \odot$ & $5.48 \mathrm{E}-05$ & $\odot . \odot \odot E+\odot \odot$ & $3.00 \mathrm{E}-04$ & 8. $00 \mathrm{E}-\odot 4$ \\
\hline Tl-209 & $4.54 \mathrm{E}+02$ & $5.48 \mathrm{E}-05$ & $\odot . \odot \odot E+\odot \odot$ & $3.00 \mathrm{E}-03$ & 2. $0 \odot \mathrm{E}-02$ \\
\hline $\mathrm{Pu}-238$ & $2.16 \mathrm{E}-05$ & $5.48 E-\odot 5$ & $\odot . \odot \odot E+\odot \odot$ & $1.00 E-\odot 6$ & 1. $\odot \odot E-\odot 4$ \\
\hline$U-234$ & 7.76E- -99 & $5.48 \mathrm{E}-05$ & $\odot . \odot \odot E+\odot \odot$ & $4.00 E-04$ & $8.00 \mathrm{E}-\odot 4$ \\
\hline Th-230 & $2.46 \mathrm{E}-08$ & $5.48 \mathrm{E}-05$ & $\odot .0 \odot E+\odot \odot$ & $5.00 \mathrm{E}-06$ & 1. $0 \odot E-04$ \\
\hline $\mathrm{Ra}-226$ & $1.19 \mathrm{E}-06$ & $5.48 E-\odot 5$ & $\odot . \odot \odot E+\odot \odot$ & $1.00 \mathrm{E}-03$ & $2 . \odot \odot E-\odot 3$ \\
\hline$R n-222$ & $1.81 \mathrm{E}-01$ & $5.48 \mathrm{E}-05$ & $\odot . \odot \odot E+\odot \odot$ & $\Theta .00 \mathrm{E}+0 \odot$ & $\odot . \odot \odot E+\odot \odot$ \\
\hline Po- 218 & $3.27 \mathrm{E}+02$ & $5.48 \mathrm{E}-05$ & $\Theta .0 \odot E+\Theta \odot$ & $4.00 E-04$ & $5.00 \mathrm{E}-03$ \\
\hline $\mathrm{Pb}-214$ & $3.72 \mathrm{E}+01$ & $5.48 E-05$ & $\odot . \odot \odot E+\odot \odot$ & $3.00 \mathrm{E}-04$ & $8.00 \mathrm{E}-\odot 4$ \\
\hline $\mathrm{Bi}-214$ & $5.02 \mathrm{E}+\odot 1$ & $5.48 \mathrm{E}-\odot 5$ & $\odot . \odot \odot E+\odot \odot$ & $1.00 \mathrm{E}-\odot 3$ & $2.0 \odot E-\odot 3$ \\
\hline Po-214 & $3.64 \mathrm{E}+08$ & $5.48 \mathrm{E}-05$ & $\odot . \odot \odot E+\odot \odot$ & $4.00 \mathrm{E}-04$ & $5.00 \mathrm{E}-03$ \\
\hline $\mathrm{Pb}-210$ & $8.51 E-05$ & $5.48 E-\odot 5$ & $\odot . \odot \odot E+\odot \odot$ & $3.0 \odot E-\odot 4$ & 8. $\odot \odot E-\odot 4$ \\
\hline $\mathrm{Bi}-210$ & 1. $38 \mathrm{E}-01$ & $5.48 E-05$ & $\odot . \odot \odot E+\odot \odot$ & $1.00 \mathrm{E}-03$ & $2.00 \mathrm{E}-\odot 3$ \\
\hline Po-210 & $5.01 \mathrm{E}-03$ & $5.48 \mathrm{E}-\odot 5$ & $\odot . \odot \odot E+\odot \odot$ & $4.00 \mathrm{E}-04$ & $5.00 \mathrm{E}-\odot 3$ \\
\hline At -218 & $2.99 \mathrm{E}+\odot 4$ & $5.48 E-\odot 5$ & $\odot . \odot \odot E+\odot \odot$ & $1.00 \mathrm{E}-\odot 2$ & 1. $\odot \odot E-\odot 2$ \\
\hline $\mathrm{Sr}-90$ & $6.52 \mathrm{E}-05$ & $5.48 \mathrm{E}-05$ & $\odot . \odot \odot E+\odot \odot$ & $2.00 \mathrm{E}-03$ & 1. $00 \mathrm{E}-02$ \\
\hline Y -90 & $2.60 \mathrm{E}-01$ & $5.48 \mathrm{E}-05$ & $\odot . \odot \odot E+\odot \odot$ & $6.00 \mathrm{E}-05$ & $2.00 \mathrm{E}-\odot 3$ \\
\hline $\mathrm{K}-40$ & $1.48 \mathrm{E}-12$ & $5.48 \mathrm{E}-\odot 5$ & $\odot . \odot \odot E+\odot \odot$ & $7.00 \mathrm{E}-\odot 3$ & 2. $\odot \odot E-\odot 2$ \\
\hline Th- 232 & $1.35 E-13$ & $5.48 E-05$ & $\odot . \odot \odot E+\odot \odot$ & $5.00 \mathrm{E}-06$ & 1. $00 \mathrm{E}-\odot 4$ \\
\hline $\mathrm{Ra}-228$ & $3.30 E-04$ & $5.48 \mathrm{E}-\odot 5$ & $\odot . \odot \odot E+\odot \odot$ & $1.00 \mathrm{E}-\odot 3$ & 2. $\odot \odot E-\odot 3$ \\
\hline Ac - 228 & $2.71 \mathrm{E}+\odot \odot$ & $5.48 \mathrm{E}-\odot 5$ & $\odot . \odot \odot E+\odot \odot$ & $2.0 \odot E-\odot 6$ & 2. $\odot \odot E-\odot 5$ \\
\hline Th - 228 & $9.92 \mathrm{E}-04$ & $5.48 \mathrm{E}-05$ & $\odot .0 \odot E+\odot \odot$ & $5.00 \mathrm{E}-06$ & 1. $00 \mathrm{E}-04$ \\
\hline $\mathrm{Ra}-224$ & $1.89 \mathrm{E}-01$ & $5.48 \mathrm{E}-05$ & $\odot . \odot \odot E+\odot \odot$ & $1.00 \mathrm{E}-03$ & $2 . \odot \odot E-\odot 3$ \\
\hline$R n-220$ & 1. $08 \mathrm{E}+03$ & $5.48 \mathrm{E}-05$ & $\odot . \odot \odot E+\odot \odot$ & $\odot .00 \mathrm{E}+\Theta \odot$ & $\odot . \odot \odot \mathrm{E}+\odot \odot$ \\
\hline Po-216 & $3.99 \mathrm{E}+05$ & $5.48 \mathrm{E}-05$ & $\odot . \odot \odot E+\odot \odot$ & $4.00 \mathrm{E}-04$ & $5.00 \mathrm{E}-03$ \\
\hline$P b-212$ & $1.56 \mathrm{E}+\odot \odot$ & $5.48 E-05$ & $\odot . \odot \odot E+\odot \odot$ & $3.00 \mathrm{E}-04$ & 8. $० \odot E-\odot 4$ \\
\hline Bi-212 & $1.65 \mathrm{E}+01$ & $5.48 \mathrm{E}-05$ & $\odot . \odot \odot \mathrm{E}+\odot \odot$ & $1.00 \mathrm{E}-03$ & $2.00 \mathrm{E}-\odot 3$ \\
\hline $\mathrm{Po}-212$ & $1.96 \mathrm{E}+11$ & $5.48 \mathrm{E}-05$ & $\odot . \odot \odot E+\odot \odot$ & $4.00 \mathrm{E}-04$ & $5.00 \mathrm{E}-03$ \\
\hline Tl-208 & $3.25 \mathrm{E}+02$ & $5.48 \mathrm{E}-\odot 5$ & $\odot . \odot \odot E+\odot \odot$ & $3.00 \mathrm{E}-\odot 3$ & 2. $\odot \odot E-\odot 2$ \\
\hline U-238 & $4.25 E-13$ & $5.48 \mathrm{E}-05$ & $\odot . \odot \odot E+\odot \odot$ & $4.0 \odot E-\odot 4$ & $8.0 \odot E-\odot 4$ \\
\hline
\end{tabular}


Appendix A

\begin{tabular}{|c|c|c|c|c|c|}
\hline Th-234 & $2.88 E-02$ & $5.48 E-05$ & $\odot . \odot \odot E+\odot \odot$ & $5 . \odot \odot E-\odot 6$ & 1. ००E-०4 \\
\hline $\mathrm{Pa}-234 \mathrm{~m}$ & $8.53 E+02$ & $5.48 E-05$ & $\odot . \odot \odot E+\odot \odot$ & $5.00 \mathrm{E}-\odot 6$ & $5.00 \mathrm{E}-06$ \\
\hline $\mathrm{Pa}-234$ & $2.48 E+\odot \odot$ & $5.48 E-05$ & $\odot . \odot \odot E+\odot \odot$ & $5 . \odot \odot E-\odot 6$ & 5. $00 \mathrm{E}-\odot 6$ \\
\hline Al-26 & $2.65 E-\odot 9$ & $5.48 E-05$ & $\odot . \odot \odot E+\odot \odot$ & 2. ๑०E-๑4 & $5.00 \mathrm{E}-04$ \\
\hline $\mathrm{Be}-7$ & 1. 3०E- $\odot 2$ & $5.48 E-05$ & $\odot . \odot \odot E+\odot \odot$ & $2 . \odot \odot E-\odot 6$ & 5. ००E-๑3 \\
\hline $\mathrm{Cm}-243$ & $6.66 E-05$ & $5.48 E-05$ & $\odot . \odot \odot E+\odot \odot$ & 2. $\odot \odot E-\odot 6$ & 2. $00 \mathrm{E}-05$ \\
\hline Am- 243 & $2.57 E-07$ & $5.48 E-05$ & $\odot . \odot \odot E+\odot \odot$ & 2. ๑०E - ०6 & $5.00 \mathrm{E}-\odot 5$ \\
\hline Np - 239 & 2. $94 \mathrm{E}-01$ & $5.48 E-05$ & $\odot . \odot \odot E+\odot \odot$ & 1. ००E- $\odot 5$ & 1. $\odot \odot E-\odot 3$ \\
\hline Pu-239 & $7.88 E-08$ & $5.48 E-05$ & $\odot . \odot \odot E+\odot \odot$ & 1. ००Е-๑6 & 1. $\odot \odot E-\odot 4$ \\
\hline$U-235$ & 2. $70 \mathrm{E}-12$ & $5.48 E-05$ & $\odot . \odot \odot E+\odot \odot$ & 4. ००Е-๑4 & 8. $.0 \mathrm{E}-\odot 4$ \\
\hline Th-231 & $6.52 E-01$ & $5.48 E-05$ & $\odot . \odot \odot E+\odot \odot$ & 5. ๑०Е - ०6 & 1. $\odot \odot E-\odot 4$ \\
\hline$P a-231$ & $5.79 E-08$ & $5.48 E-05$ & $\odot . \odot \odot E+\odot \odot$ & 5. ००E - ०6 & $5.00 \mathrm{E}-06$ \\
\hline$A c-227$ & $8.71 E-05$ & $5.48 E-05$ & $\odot . \odot \odot E+\odot \odot$ & 2. $\odot ० E-\odot 6$ & 2. $00 \mathrm{E}-\odot 5$ \\
\hline Th- 227 & $3.70 E-\odot 2$ & $5.48 E-05$ & $\odot . \odot \odot E+\odot \odot$ & $5 . \odot \odot E-\odot 6$ & 1. $.0 \mathrm{E}-\odot 4$ \\
\hline $\mathrm{Ra}-223$ & $6.06 E-02$ & $5.48 E-05$ & $\odot . \odot \odot E+\odot \odot$ & 1. $00 \mathrm{E}-03$ & 2. .०० - ०3 \\
\hline Rn- 219 & $1.51 E+04$ & $5.48 E-05$ & $\odot . \odot \odot E+\odot \odot$ & $\odot . \odot \odot E+\odot \odot$ & $\odot . \odot \odot E+\odot \odot$ \\
\hline Po- 215 & $3.36 E+07$ & $5.48 E-05$ & $\odot . \odot \odot E+\odot \odot$ & $4 . \odot \odot E-\odot 4$ & 5. ००E- - \\
\hline $\mathrm{Pb}-211$ & $2.76 E+01$ & $5.48 E-05$ & $\odot . \odot \odot E+\odot \odot$ & 3. $00 \mathrm{E}-04$ & 8. $00 \mathrm{E}-04$ \\
\hline Bi-211 & $4.66 E+02$ & $5.48 E-05$ & $\odot . \odot \odot E+\odot \odot$ & 1. $00 \mathrm{E}-03$ & 2. .०० - ०3 \\
\hline Tl - 207 & 2. $09 \mathrm{E}+02$ & $5.48 E-05$ & $\odot . \odot \odot E+\odot \odot$ & 3. ००E - ०3 & 2. $\odot \odot E-\odot 2$ \\
\hline Po- 211 & $1.16 \mathrm{E}+05$ & $5.48 E-05$ & $\odot . \odot \odot E+\odot \odot$ & $4.0 \odot E-\odot 4$ & 5. ००E-०3 \\
\hline $\mathrm{Fr}-223$ & $4.58 E+01$ & $5.48 E-05$ & $\odot . \odot \odot E+\odot \odot$ & 8. ००E-०3 & 3. ००E-०2 \\
\hline Co-58 & $9.79 E-03$ & $5.48 E-05$ & $\odot . \odot \odot E+\odot \odot$ & 2. ००E - ०3 & 3. $0 \odot \mathrm{E}-02$ \\
\hline Co- 60 & $3.60 E-\odot 4$ & $5.48 E-05$ & $\odot . \odot \odot E+\odot \odot$ & $2 . \odot \odot E-\odot 3$ & 3. ००E-๑2 \\
\hline Cs - 134 & $9.20 E-04$ & $5.48 E-05$ & $\odot . \odot \odot E+\odot \odot$ & 1. $00 \mathrm{E}-02$ & 5. $00 \mathrm{E}-02$ \\
\hline Eu-152 & $1.32 \mathrm{E}+00$ & $5.48 E-05$ & $\odot . \odot \odot E+\odot \odot$ & $6.00 \mathrm{E}-\odot 5$ & $2.0 \odot E-\odot 3$ \\
\hline Gd-152 & $1.76 \mathrm{E}-17$ & $5.48 E-05$ & $\odot . \odot \odot E+\odot \odot$ & $6.00 \mathrm{E}-05$ & 2. ००E-०3 \\
\hline Eu-154 & $1.78 \mathrm{E}+\odot \odot$ & $5.48 E-05$ & $\odot . \odot \odot E+\odot \odot$ & $6.0 \odot E-\odot 5$ & $2 . \odot \odot E-\odot 3$ \\
\hline Eu-155 & $2.16 E-04$ & $5.48 E-05$ & $\odot . \odot \odot E+\odot \odot$ & $6.00 \mathrm{E}-05$ & 2. .०० - ०3 \\
\hline Nb - 94 & $9.35 E-\odot 8$ & $5.48 E-05$ & $\odot . \odot \odot E+\odot \odot$ & $2 . \odot \odot E-\odot 6$ & 3. ००E-๑7 \\
\hline Sb-125 & $6.85 E-04$ & $5.48 E-05$ & $\odot . \odot \odot E+\odot \odot$ & 1. ००E-०4 & 1. ००E-०3 \\
\hline Te-125m & 1. $20 \mathrm{E}-\odot 2$ & $5.48 E-05$ & $\odot . \odot \odot E+\odot \odot$ & $5 . \odot \odot E-\odot 4$ & 7. ००E-๑3 \\
\hline $\mathrm{H}-3$ & $1.54 \mathrm{E}-04$ & $5.48 E-05$ & $\odot . \odot \odot E+\odot \odot$ & $\odot . \odot \odot E+\odot \odot$ & $\odot . \odot \odot E+\odot \odot$ \\
\hline
\end{tabular}

FOOTNOTES :

(1) Fraction of animal's daily intake of nuclide which appears in each $L$ of milk (days/L)

(2) Fraction of animal's daily intake of nuclide which appears in each $\mathrm{kg}$ of meat (days $/ \mathrm{kg}$ ) 


\section{VALUES FOR RADIONUCLIDE-DEPENDENT PARAMETERS}

\begin{tabular}{|c|c|c|c|c|}
\hline \multirow[b]{2}{*}{ Nuclide } & \multicolumn{2}{|c|}{$\begin{array}{l}\text { CONCENTRATION } \\
\text { UPTAKE FACTOR }\end{array}$} & \multicolumn{2}{|c|}{ GI UPTAKE FRACTION } \\
\hline & Forage (1) & Edible (2) & Inhalation & Ingestion \\
\hline Cs -137 & 1. $\odot \odot E+\odot \odot$ & 2. ००E - ०1 & 1. $\odot \odot E+\odot \odot$ & 1. $\odot \odot E+\odot \odot$ \\
\hline $\mathrm{Ba}-137 \mathrm{~m}$ & 1. ๑७E- $० 1$ & 1. ००E - ०2 & 2. $\odot \odot E-\odot 1$ & 2. ๑๑E - ๑1 \\
\hline Am-241 & 1. $.0 \mathrm{E}-01$ & 1. ००E-०3 & $5.00 E-\odot 4$ & $5.0 \odot E-\odot 4$ \\
\hline$N p-237$ & 1. ๑๑E - ०1 & 2. ๑०E - ०2 & 5. ०७Е - ๑4 & 5. ๑७Е - ๑4 \\
\hline $\mathrm{Pa}-233$ & 1. ๑๑E- $९ 1$ & 1. ००E - ०2 & 5. ०७Е - ๑4 & 5. ๑७Е - ๑4 \\
\hline$U-233$ & 1. $00 \mathrm{E}-01$ & $2.00 E-\odot 3$ & 2. .०० - ०2 & $2.0 \odot E-\odot 2$ \\
\hline Th - 229 & 1. ๑७E- $९ 1$ & 1. ००E-๑3 & 5. ००Е-๑4 & 5. ๑७Е - ๑4 \\
\hline $\mathrm{Ra}-225$ & 2. ๑७E - ०1 & 4. ००E - ०2 & 2. $\odot \odot E-\odot 1$ & 2. ๑७E - ๑1 \\
\hline$A c-225$ & 1. ๑๑E- ०1 & 1. ००E- - & 5. ००E - ๑4 & 5. ๑०Е-๑4 \\
\hline Fr -221 & 1. $\odot \odot E-\odot 1$ & 3. ००E - ०2 & 1. $\odot \odot E+\odot \odot$ & 1. $\odot \odot E+\odot \odot$ \\
\hline At -217 & 9. ๑७Е- -1 & 2. .०E - ०1 & 1. $\odot \odot E+\odot \odot$ & 1. $\odot \odot E+\odot \odot$ \\
\hline $\mathrm{Bi}-213$ & 5. ๑७Е - ๑1 & 1. $00 \mathrm{E}-01$ & 5. ००Е-๑2 & 5. ๑७Е - ๑2 \\
\hline Po- 213 & 1. ००Е-०1 & 1. ००Е-๑3 & 1. ००E- $\odot 1$ & 1. ००Е- $\odot 1$ \\
\hline Pb - 209 & 1. ०७Е- -1 & 4. ००E - ๑3 & 2. ००E - ०1 & 2. ๑०E - ०1 \\
\hline T1-209 & 6. ๑७Е - ०1 & 2. $0 \odot E-\odot 1$ & 1. ००E+०० & 1. ००E+०० \\
\hline Pu- 238 & 1. ๑๑E- -1 & 1. ००E-०3 & 5. ००Е - ๑४ & 5. ๑९Е - ๑4 \\
\hline$U-234$ & 1. $\odot \odot E-\odot 1$ & 2. ००E-๑3 & 2. ००E-๑2 & 2. ๑०Е-๑2 \\
\hline Th - 230 & 1. ๑७Е- -1 & 1. ००Е - ०3 & 5. ००Е-๑4 & 5. ๑७Е - ๑4 \\
\hline $\mathrm{Ra}-226$ & 2. ००Е-०1 & 4. ๑०Е-๑2 & 2. $\odot \odot E-\odot 1$ & 2. ๑०Е- $\odot 1$ \\
\hline$R n-222$ & $\odot . \odot \odot E+\odot \odot$ & $\odot . \odot \odot E+\odot \odot$ & $\odot . \odot \odot E+\odot \odot$ & $\odot . \odot \odot E+\odot \odot$ \\
\hline Po- 218 & 1. ००E-०1 & 1. ००E-०3 & 1. $\odot \odot E-\odot 1$ & 1. $\odot \odot E-\odot 1$ \\
\hline $\mathrm{Pb}-214$ & 1. ००Е- 01 & 4. ००E- - & 2. $.0 \mathrm{E}-\odot 1$ & 2. ००E- - \\
\hline Bi-214 & 5. ००E- 01 & 1. ००E- 01 & $5.00 E-02$ & $5.0 \odot E-\odot 2$ \\
\hline Po- 214 & 1. $\odot \odot E-\odot 1$ & 1. ००E-๑3 & 1. $\odot \odot E-\odot 1$ & 1. $\odot \odot E-\odot 1$ \\
\hline $\mathrm{Pb}-210$ & 1. ००E- 01 & $4.00 E-\odot 3$ & 2. $00 \mathrm{E}-01$ & 2. .०E - 01 \\
\hline $\mathrm{Bi}-210$ & 5. $. \odot E-01$ & 1. $\odot \odot E-\odot 1$ & 5. ००E- - & 5. ००E - ०2 \\
\hline Po- 210 & 1. ००E- 01 & 1. ००E-०3 & $1.00 \mathrm{E}-01$ & 1. ००E- 01 \\
\hline At -218 & 9. ००Е- 01 & 2. ००E - ०1 & 1. $\odot \odot E+\odot \odot$ & 1. $\odot \odot E+\odot \odot$ \\
\hline Sr-90 & 4. .०E+०० & 3. 00E - 01 & 3. ००E - 01 & 3. ००E - 01 \\
\hline$Y-90$ & 1. $. \odot E-\odot 1$ & 2. .०E - ०3 & 1. ००E- - ४ & 1. ००E- $\odot 4$ \\
\hline$K-40$ & 3. ००E+०० & 3. $00 \mathrm{E}-01$ & 1. ००E+०० & 1. ००E+०० \\
\hline Th- 232 & 1. $0 \odot \mathrm{E}-01$ & 1. $00 \mathrm{E}-03$ & 5. $0 \odot \mathrm{E}-\odot 4$ & 5. ००E- - 4 \\
\hline $\mathrm{Ra}-228$ & 2. ००E- 01 & $4.00 E-\odot 2$ & 2. $00 \mathrm{E}-\odot 1$ & 2. ००E - 01 \\
\hline$A c-228$ & 1. ०७Е- $९ 1$ & 1. ००E-०3 & 5. ००Е - ๑४ & 5. ๑७Е - ๑४ \\
\hline Th - 228 & 1. ००E- 01 & 1. ००E-०3 & $5.00 E-\odot 4$ & 5. ००Е- - \\
\hline $\mathrm{Ra}-224$ & 2. ๑७E - ०1 & 4. .०E - ०2 & 2. $\odot \odot E-\odot 1$ & 2. $0 \odot \mathrm{E}-01$ \\
\hline$R n-220$ & ๑ . ०७Е+०० & $\odot . \odot \odot E+\odot \odot$ & $\odot . \odot \odot E+\odot \odot$ & $\odot . \odot \odot E+\odot \odot$ \\
\hline Po- 216 & 1. ०७Е- ०1 & 1. ००E-०3 & 1. ००E - ०1 & 1. ๑๑Е - ๑1 \\
\hline $\mathrm{Pb}-212$ & 1. ००Е- 01 & $4.00 E-\odot 3$ & 2. $0 \odot E-\odot 1$ & 2. ००E - 01 \\
\hline $\mathrm{Bi}-212$ & 5. ๑७Е - ๑1 & 1. $\odot \odot E-\odot 1$ & 5. ๑७Е - ๑2 & 5. ๑७Е - ๑2 \\
\hline Po- 212 & 1. ๑७Е- -1 & 1. ००E-०3 & 1. ००Е- -1 & 1. ๑๑Е - ๑1 \\
\hline $\mathrm{Tl}-208$ & 6. ๑०E- - & 2. $\odot \odot E-\odot 1$ & 1. $\odot \odot E+\odot \odot$ & 1. $\odot \odot E+\odot \odot$ \\
\hline U-238 & 1. ๑७Е-०1 & 2. ००E - ๑3 & 2. ००E-๑2 & 2. ๑७Е-๑2 \\
\hline
\end{tabular}




\begin{tabular}{|c|c|c|c|c|}
\hline Th-234 & 1. ००E - 01 & 1. ००E- - & 5. ००E - ०4 & $5.0 \odot E-\odot 4$ \\
\hline$P a-234 m$ & 1. . ००E-०1 & 1. ००E-०2 & 5. $00 \mathrm{E}-\odot 4$ & $5 . \odot \odot E-\odot 4$ \\
\hline $\mathrm{Pa}-234$ & 1. ००E- ०1 & 1. $00 \mathrm{E}-02$ & $5.00 E-\odot 4$ & $5.00 E-\odot 4$ \\
\hline Al- 26 & 1. ๑७E- $९ 1$ & 4. ००E-๑3 & 1. ००E - ०2 & 1. $\odot \odot E-\odot 2$ \\
\hline $\mathrm{Be}-7$ & 1. ๑๑Е-๑1 & $4 . \odot \odot E-\odot 3$ & 5. ๑७Е - ๑3 & 5. ००Е-๑3 \\
\hline $\mathrm{Cm}-243$ & 1. ००E-०1 & 1. .०० - ०3 & $5.00 E-\odot 4$ & $5.00 E-04$ \\
\hline Am- 243 & 1. ००E- ०1 & 1. .0०E-०3 & $5.00 E-\odot 4$ & $5.0 \odot E-\odot 4$ \\
\hline$N p-239$ & 1. ๑०Е- ०1 & 2. ००E-๑2 & 5. ๑०E - ๑4 & 5. ००E-๑4 \\
\hline Pu- 239 & 1. ००E-०1 & 1. .०० - ०3 & $5.00 E-\odot 4$ & $5.0 \odot E-\odot 4$ \\
\hline$U-235$ & 1. ๑०Е-०1 & 2. .०Е-०3 & $2 . \odot \odot E-\odot 2$ & 2. $\odot \odot E-\odot 2$ \\
\hline Th-231 & 1. ००E-०1 & 1. .०० - ०3 & $5.00 E-\odot 4$ & $5.00 E-\odot 4$ \\
\hline $\mathrm{Pa}-231$ & 1. .०० - 01 & 1. ००E-०2 & 5. ००E - 04 & $5.00 E-04$ \\
\hline$A C-227$ & 1. .०० - 01 & 1. ००E-๑3 & 5. ००E - ๑4 & 5. ๑७E- - ४4 \\
\hline Th-227 & 1. .०० - 01 & 1. ००E-๑3 & 5. ००E - ๑4 & 5. ๑७E - ๑4 \\
\hline $\mathrm{Ra}-223$ & 2. ००E-०1 & 4. .०० - ०2 & 2. .०E - ०1 & 2. . $00 \mathrm{E}-01$ \\
\hline Rn- 219 & $\odot . \odot \odot E+\odot \odot$ & $\odot . \odot \odot E+\odot \odot$ & $\odot . \odot \odot E+\odot \odot$ & $\odot . \odot \odot E+\odot \odot$ \\
\hline Po- 215 & 1. ๑๑Е- ०1 & 1. $.0 \mathrm{E}-\odot 3$ & 1. $\odot \odot E-\odot 1$ & 1. $. \odot E-\odot 1$ \\
\hline $\mathrm{Pb}-211$ & 1. ००E-०1 & 4. .०Е- - ०3 & 2. $.0 \mathrm{E}-01$ & 2. . $00 \mathrm{E}-01$ \\
\hline Bi-211 & 5. ००Е- 01 & 1. $00 \mathrm{E}-01$ & $5.00 \mathrm{E}-02$ & $5.0 \odot E-02$ \\
\hline Tl- 207 & 6. ००Е- 01 & 2. $00 \mathrm{E}-01$ & 1. $.0 \mathrm{E}+0 \odot$ & 1. $.0 \mathrm{E}+0 \odot$ \\
\hline Po- 211 & 1. ००Е-०1 & 1. .०० - ०3 & 1. $00 \mathrm{E}-01$ & 1. $00 \mathrm{E}-01$ \\
\hline Fr - 223 & 1. ००E-०1 & 3. $00 \mathrm{E}-02$ & 1. $\odot ० E+\odot \odot$ & 1. $.0 \mathrm{E}+00$ \\
\hline $\mathrm{Co}-58$ & 2. $\odot \odot E+\odot \odot$ & 8. $00 \mathrm{E}-02$ & 1. $00 \mathrm{E}-01$ & 1. $00 \mathrm{E}-01$ \\
\hline $\mathrm{Co}-60$ & $2 . \odot \odot E+\odot \odot$ & 8. ००E-๑2 & 1. $\odot \odot E-\odot 1$ & 1. $\odot \odot E-\odot 1$ \\
\hline Cs - 134 & 1. $\odot \odot E+\odot \odot$ & 2. $00 \mathrm{E}-01$ & 1. $.0 \mathrm{E}+0 \odot$ & 1. $00 \mathrm{E}+0 \odot$ \\
\hline Eu-152 & 1. $\odot \odot E-\odot 1$ & $2.0 \odot E-\odot 3$ & $5.00 \mathrm{E}-\odot 4$ & $5.0 \odot E-\odot 4$ \\
\hline Gd-152 & 1. ००E-०1 & 2. ००E-०3 & $5 . \odot \odot E-\odot 4$ & $5.0 \odot E-\odot 4$ \\
\hline Eu-154 & 1. ๑०Е-๑1 & $2 . \odot \odot E-\odot 3$ & 5. ๑०E - ๑4 & $5 . \odot \odot E-\odot 4$ \\
\hline Eu-155 & 1. $0 \odot E-01$ & 2. ००E-०3 & $5.00 \mathrm{E}-04$ & $5.00 \mathrm{E}-04$ \\
\hline $\mathrm{Nb}-94$ & 1. ๑०Е-०1 & 1. $. \odot E-\odot 2$ & 1. $\odot \odot E-\odot 2$ & 1. $\odot \odot E-\odot 2$ \\
\hline Sb-125 & 1. $.0 \mathrm{E}-01$ & 1. ००E- 02 & 1. $.0 \mathrm{E}-01$ & 1. .०Е- - 1 \\
\hline Te-125m & $1.30 E+\odot \odot$ & 1. $. \odot E-\odot 1$ & 3. ๑०E - ०1 & 3. ๑०E - ०1 \\
\hline $\mathrm{H}-3$ & $\odot . \odot \odot E+\odot \odot$ & $\odot . \odot \odot E+\odot \odot$ & 1. $\odot \odot E+\odot \odot$ & 1. $\odot \odot E+\odot \odot$ \\
\hline
\end{tabular}

FOOTNOTES: (1) Concentration factor for uptake of nuclide from soil for pasture and forage (in $\mathrm{pCi} / \mathrm{kg}$ dry weight per $\mathrm{pCi} / \mathrm{kg}$ dry soil)

(2) Concentration factor for uptake of nuclide from soil by edible parts of crops (in $\mathrm{pCi} / \mathrm{kg}$ wet weight per $\mathrm{pCi} / \mathrm{kg}$ dry soil) 
Appendix A

Nov 27, $2006 \quad 05: 40$ pm

GENERAL

Page 4

DECAY CHAIN ACTIVITIES

\begin{tabular}{|c|c|c|c|c|}
\hline Nuclide & Stack & Activity at 500 . & seconds Activity at & 100.00 years \\
\hline Cs -137 & 1 & $8.7600 \mathrm{E}-06$ & $2.9690 \mathrm{E}-06$ & \\
\hline $\mathrm{Ba}-137 \mathrm{~m}$ & 1 & $8.3360 E-06$ & $2.8070 \mathrm{E}-\odot 6$ & \\
\hline Am-241 & 1 & $1.1400 \mathrm{E}-05$ & $3.8820 E-\odot 6$ & \\
\hline $\mathrm{Np}-237$ & 1 & $\odot . \odot \odot \odot \odot E+\odot \odot$ & $\odot . \odot \odot \odot \odot E+\odot \odot$ & \\
\hline $\mathrm{Pa}-233$ & 1 & $\odot . \odot \odot \odot \odot E+\odot \odot$ & $\odot . \odot \odot \odot \odot E+\odot \odot$ & \\
\hline$U-233$ & 1 & $\odot . \odot \odot \odot \odot E+\odot \odot$ & $\odot . \odot \odot \odot \odot E+\odot \odot$ & \\
\hline Th-229 & 1 & $\odot .000 \odot \mathrm{E}+\odot \odot$ & $\odot .000 \odot \mathrm{E}+\odot \odot$ & \\
\hline $\mathrm{Ra}-225$ & 1 & $\odot . \odot \odot \odot \odot E+\odot \odot$ & $\odot . \odot \odot \odot \odot E+\odot \odot$ & \\
\hline Ac -225 & 1 & $\odot . \odot \odot \odot \odot E+\odot \odot$ & $\odot . \odot \odot \odot \odot E+\odot \odot$ & \\
\hline $\mathrm{Fr}-221$ & 1 & $\odot . \odot \odot \odot \odot E+\odot \odot$ & $\odot . \odot \odot \odot \odot E+\odot \odot$ & \\
\hline At -217 & 1 & $\odot . \odot \odot \odot \odot E+\odot \odot$ & $\odot . \odot \odot \odot \odot E+\odot \odot$ & \\
\hline $\mathrm{Bi}-213$ & 1 & $\odot . \odot \odot \odot \odot E+\odot \odot$ & $\odot . \odot \odot \odot \odot E+\odot \odot$ & \\
\hline Po- 213 & 1 & $\odot . \odot \odot \odot \odot E+\odot \odot$ & $\odot . \odot \odot \odot \odot E+\odot \odot$ & \\
\hline$P b-209$ & 1 & $\odot . \odot \odot \odot \odot E+\odot \odot$ & $\odot . \odot \odot \odot \odot E+\odot \odot$ & \\
\hline Tl-209 & 1 & $\odot . \odot \odot \odot \odot E+\odot \odot$ & $\odot . \odot \odot \odot \odot E+\odot \odot$ & \\
\hline Pu-238 & 1 & $8.200 \odot \mathrm{E}-07$ & $2.7880 \mathrm{E}-07$ & \\
\hline$U-234$ & 1 & $4.810 \odot E-\odot 5$ & $1.6380 \mathrm{E}-05$ & \\
\hline Th-230 & 1 & $4.8100 \mathrm{E}-05$ & $1.6380 \mathrm{E}-05$ & \\
\hline $\mathrm{Ra}-226$ & 1 & $4.810 \odot E-\odot 5$ & $1.6380 \mathrm{E}-05$ & \\
\hline$R n-222$ & 1 & $4.8100 \mathrm{E}-\odot 5$ & $1.6370 \mathrm{E}-05$ & \\
\hline Po- 218 & 1 & $4.8100 \mathrm{E}-05$ & $1.6370 \mathrm{E}-05$ & \\
\hline$P b-214$ & 1 & 4. $810 \odot E-\odot 5$ & $1.6370 \mathrm{E}-05$ & \\
\hline $\mathrm{Bi}-214$ & 1 & 4. 8100E- $\odot 5$ & $1.6370 \mathrm{E}-05$ & \\
\hline Po- 214 & 1 & 4. $8090 E-05$ & $1.6370 \mathrm{E}-05$ & \\
\hline $\mathrm{Pb}-210$ & 1 & $4.8100 \mathrm{E}-05$ & 1.6380E- 05 & \\
\hline $\mathrm{Bi}-210$ & 1 & $4.8100 \mathrm{E}-\odot 5$ & $1.6370 \mathrm{E}-05$ & \\
\hline Po- 210 & 1 & $4.8100 E-05$ & $1.6380 \mathrm{E}-05$ & \\
\hline At -218 & 1 & $9.620 \odot \mathrm{E}-\odot 9$ & $\odot . \odot \odot \odot \odot E+\odot \odot$ & \\
\hline Sr - $9 \odot$ & 1 & $3.100 \odot E-\odot 6$ & 1. $0500 \mathrm{E}-06$ & \\
\hline$Y-90$ & 1 & $3.1000 \mathrm{E}-06$ & $1.0500 \mathrm{E}-06$ & \\
\hline $\mathrm{K}-4 \odot$ & 1 & $2.880 \odot E-\odot 4$ & $9.8100 \mathrm{E}-05$ & \\
\hline Th-232 & 1 & $2.9600 \mathrm{E}-\odot 5$ & 1. $0080 \mathrm{E}-05$ & \\
\hline $\mathrm{Ra}-228$ & 1 & $2.9600 \mathrm{E}-\odot 5$ & 1. $0080 \mathrm{E}-\odot 5$ & \\
\hline Ac- -228 & 1 & $2.960 \odot E-\odot 5$ & 1. $0 \odot 80 E-\odot 5$ & \\
\hline Th-228 & 1 & $2.9600 \mathrm{E}-\odot 5$ & 1. $0080 \mathrm{E}-05$ & \\
\hline $\mathrm{Ra}-224$ & 1 & $2.960 \odot E-\odot 5$ & 1. $.0880 E-05$ & \\
\hline$R n-220$ & 1 & $2.960 \odot E-\odot 5$ & 1. $0 \odot 80 E-\odot 5$ & \\
\hline Po- 216 & 1 & $2.9600 \mathrm{E}-\odot 5$ & 1. $0080 \mathrm{E}-05$ & \\
\hline$P b-212$ & 1 & $2.9600 \mathrm{E}-\odot 5$ & 1. $0080 \mathrm{E}-05$ & \\
\hline $\mathrm{Bi}-212$ & 1 & $2.9600 \mathrm{E}-\odot 5$ & 1. $0 \odot 80 E-05$ & \\
\hline Po- 212 & 1 & $1.8960 \mathrm{E}-05$ & $6.4560 \mathrm{E}-06$ & \\
\hline Tl-208 & 1 & 1. $065 \odot E-\odot 5$ & $3.6200 \mathrm{E}-\odot 6$ & \\
\hline$U-238$ & 1 & $4.8100 \mathrm{E}-05$ & $1.6380 \mathrm{E}-05$ & \\
\hline Th-234 & 1 & $4.8100 \mathrm{E}-05$ & $1.6380 \mathrm{E}-05$ & \\
\hline $\mathrm{Pa}-234 \mathrm{~m}$ & 1 & 4. . 8०००E-๑5 & $1.6340 \mathrm{E}-05$ & \\
\hline $\mathrm{Pa}-234$ & 1 & $6.2500 \mathrm{E}-\odot 8$ & $\odot . \odot \odot \odot \odot E+\odot \odot$ & \\
\hline Al-26 & 1 & $1.2800 \mathrm{E}-06$ & $4.3600 \mathrm{E}-07$ & \\
\hline
\end{tabular}




\begin{tabular}{|c|c|c|c|}
\hline $\mathrm{Be}-7$ & 1 & 8. 4490E- - & 1. $2920 \mathrm{E}-06$ \\
\hline $\mathrm{Cm}-243$ & 1 & $6.9400 \mathrm{E}-06$ & $2.3510 \mathrm{E}-06$ \\
\hline Am-243 & 1 & $\odot . \odot \odot \odot \odot E+\odot \odot$ & $\odot . \odot \odot \odot \odot E+\odot \odot$ \\
\hline$N p-239$ & 1 & $\odot . \odot \odot \odot \odot E+\odot \odot$ & $\odot . \odot \odot \odot \odot E+\odot \odot$ \\
\hline Pu-239 & 1 & $3.260 \odot E-\odot 5$ & $1.1100 \mathrm{E}-05$ \\
\hline$U-235$ & 1 & $6.7300 \mathrm{E}-05$ & $2.2920 \mathrm{E}-05$ \\
\hline Th-231 & 1 & $6.7300 \mathrm{E}-05$ & $2.2910 \mathrm{E}-05$ \\
\hline $\mathrm{Pa}-231$ & 1 & $6.7300 \mathrm{E}-\odot 5$ & $2.2920 E-05$ \\
\hline Ac -227 & 1 & $6.7300 \mathrm{E}-05$ & $2.2920 \mathrm{E}-05$ \\
\hline Th- 227 & 1 & $6.6400 \mathrm{E}-05$ & $2.2600 \mathrm{E}-05$ \\
\hline $\mathrm{Ra}-223$ & 1 & $6.7300 \mathrm{E}-05$ & $2.2850 \mathrm{E}-05$ \\
\hline$R n-219$ & 1 & $6.7300 \mathrm{E}-05$ & $2.2850 \mathrm{E}-05$ \\
\hline Po- 215 & 1 & $6.7300 \mathrm{E}-05$ & $2.2850 \mathrm{E}-05$ \\
\hline$P b-211$ & 1 & $6.730 \odot E-\odot 5$ & $2.2850 E-05$ \\
\hline $\mathrm{Bi}-211$ & 1 & $6.7300 \mathrm{E}-05$ & $2.2850 \mathrm{E}-05$ \\
\hline Tl-207 & 1 & $6.7110 \mathrm{E}-05$ & $2.2780 \mathrm{E}-05$ \\
\hline Po- 211 & 1 & $1.884 \odot \mathrm{E}-\odot 7$ & $\odot . \odot \odot \odot \odot E+\odot \odot$ \\
\hline$F r-223$ & 1 & $9.3970 \mathrm{E}-07$ & $3.1610 \mathrm{E}-07$ \\
\hline Co-58 & 1 & $6.40 \odot \odot \mathrm{E}-\odot 7$ & $7.2630 \mathrm{E}-08$ \\
\hline Co-60 & 1 & 1. 2100E-๑6 & $4.0060 \mathrm{E}-07$ \\
\hline Cs -134 & 1 & $5.7600 \mathrm{E}-\odot 6$ & $1.8260 \mathrm{E}-06$ \\
\hline Eu-152 & 1 & $7.1450 E-\odot 6$ & $6.6930 \mathrm{E}-\odot 9$ \\
\hline Gd-152 & 1 & $\odot . \odot \odot \odot \odot E+\odot \odot$ & $\odot . \odot \odot \odot \odot E+\odot \odot$ \\
\hline Eu-154 & 1 & $5.5330 \mathrm{E}-06$ & $2.5710 \mathrm{E}-\odot 9$ \\
\hline Eu-155 & 1 & $3.470 \odot E-\odot 6$ & $1.1620 E-\odot 6$ \\
\hline $\mathrm{Nb}-94$ & 1 & 1. $080 \odot E-\odot 6$ & $3.6790 \mathrm{E}-07$ \\
\hline$S b-125$ & 1 & $2.9100 \mathrm{E}-\odot 6$ & $9.3920 \mathrm{E}-07$ \\
\hline $\mathrm{Te}-125 \mathrm{~m}$ & 1 & $\odot . \odot \odot \odot \odot E+\odot \odot$ & $7.8250 \mathrm{E}-\odot 8$ \\
\hline $\mathrm{H}-3$ & 1 & 1. $30 \odot \odot E-\odot 5$ & $4.3740 E-\odot 6$ \\
\hline
\end{tabular}




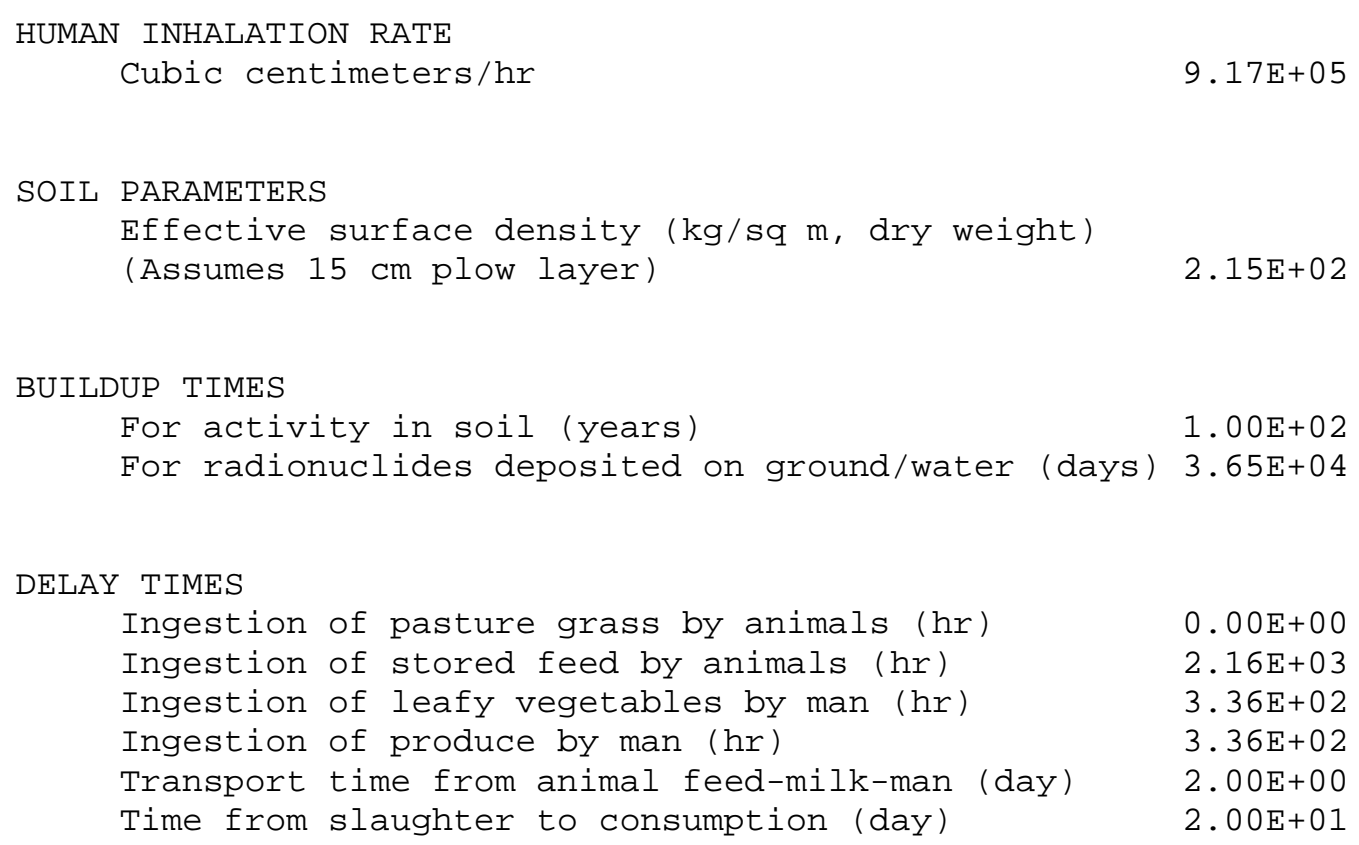


VALUES FOR RADIONUCLIDE-INDEPENDENT PARAMETERS

\begin{tabular}{|c|c|}
\hline Contaminated feed/forage ( $\mathrm{kg} /$ day, dry weight) & $1.56 \mathrm{E}+01$ \\
\hline $\begin{array}{l}\text { DAIRY PRODUCTIVITY } \\
\text { Milk production of cow (L/day) }\end{array}$ & 1. $10 \mathrm{E}+\odot 1$ \\
\hline $\begin{array}{l}\text { MEAT ANIMAL SLAUGHTER PARAMETERS } \\
\text { Muscle mass of animal at slaughter }(\mathrm{kg}) \\
\text { Fraction of herd slaughtered (per day) }\end{array}$ & $\begin{array}{l}2.0 \odot \mathrm{E}+\odot 2 \\
3.81 \mathrm{E}-\odot 3\end{array}$ \\
\hline $\begin{array}{l}\text { DECONTAMINATION } \\
\text { Fraction of radioactivity retained after washing } \\
\text { for leafy vegetables and produce }\end{array}$ & $5.0 \odot E-\odot 1$ \\
\hline $\begin{array}{l}\text { FRACTIONS GROWN IN GARDEN OF INTEREST } \\
\text { Produce ingested } \\
\text { Leafy vegetables ingested }\end{array}$ & $\begin{array}{l}1 . \odot \odot E+\odot \odot \\
1.0 \odot E+\odot \odot\end{array}$ \\
\hline $\begin{array}{l}\text { INGESTION RATIOS: } \\
\text { IMMEDIATE SURROUNDING AREA/TOTAL WITHIN AREA } \\
\text { Vegetables } \\
\text { Meat } \\
\text { Milk }\end{array}$ & $\begin{array}{l}\text { 7. } \odot \odot E-\odot 1 \\
4.4 \odot E-\odot 1 \\
4.0 \odot E-\odot 1\end{array}$ \\
\hline
\end{tabular}

MINIMUM INGESTION FRACTIONS FROM OUTSIDE AREA (Minimum fractions of food types from outside area listed below are actual fixed values.) Vegetables Meat

Milk

$\odot . \odot \odot E+\odot \odot$

$\odot . \odot \odot E+\odot \odot$

$\odot . \odot \odot E+\odot \odot$

HUMAN FOOD UTILIZATION FACTORS

Produce ingestion $(\mathrm{kg} / \mathrm{y}) \quad 1.76 \mathrm{E}+\odot 2$

Milk ingestion ( $\mathrm{L} / \mathrm{y}$ )

Meat ingestion $(\mathrm{kg} / \mathrm{y})$

$1.12 \mathrm{E}+02$

8. $50 \mathrm{E}+01$

Leafy vegetable ingestion ( $\mathrm{kg} / \mathrm{y})$

$1.80 \mathrm{E}+01$

SWIMMING PARAMETERS

Fraction of time spent swimming $\quad 0.00 \mathrm{E}+\odot \odot$

Dilution factor for water $(\mathrm{cm}) \quad 1.00 \mathrm{E}+\Theta \odot$ 


\section{Appendix B}

\section{NARAC Modeling Input and Output for the Proposed Divine Strake Experiment}




\section{Example Scenario Assumptions}

- Explosive Geometry: provided by requestor

- $1000 \mathrm{~m}$ height, $300 \mathrm{~m}$ radius

- Release Times: 1600 UTC, 15 Jan 2005 and 20 Jan 2006

- Source Amount: provided by requestor

- All material released in respirable sized particles

- Location: provided by requestor

- 37.02348 N 116.181963 W

- Weather: Meteorological observations

- NTS and WMO stations beginning 1600 UTC 15 Jan 2005

- NTS and WMO stations beginning 1600 UTC 20 Jan 2006

- Population Estimates: LANL Day/Night Population

- Terrain Source: DTED (Digital Terrain Elevation Data)

- Models used: LLNL/NARAC 


\section{Jan 15, 2005 - surface winds at release start}

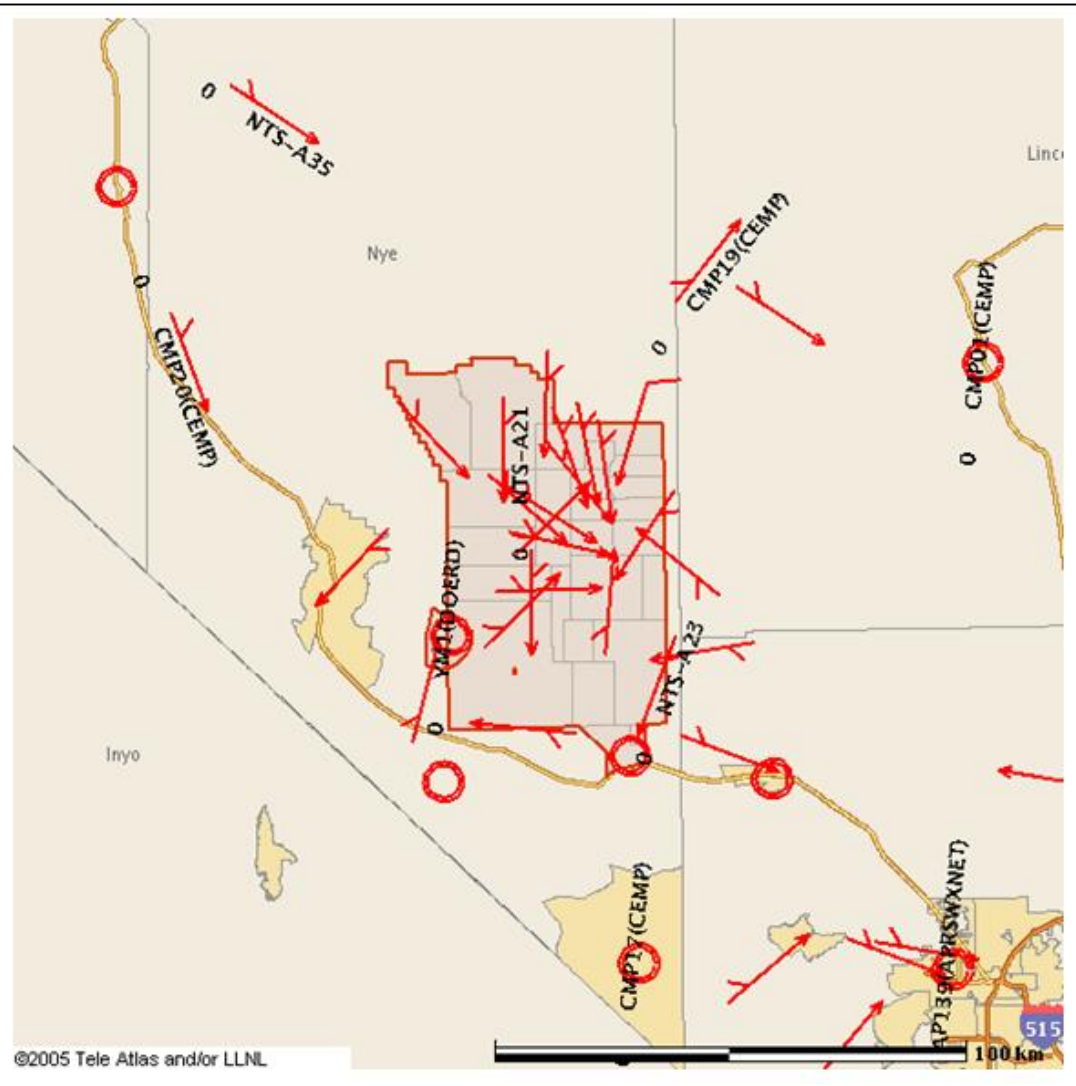


Appendix B

\section{Jan 15, 2005 - midlevel winds at release start}

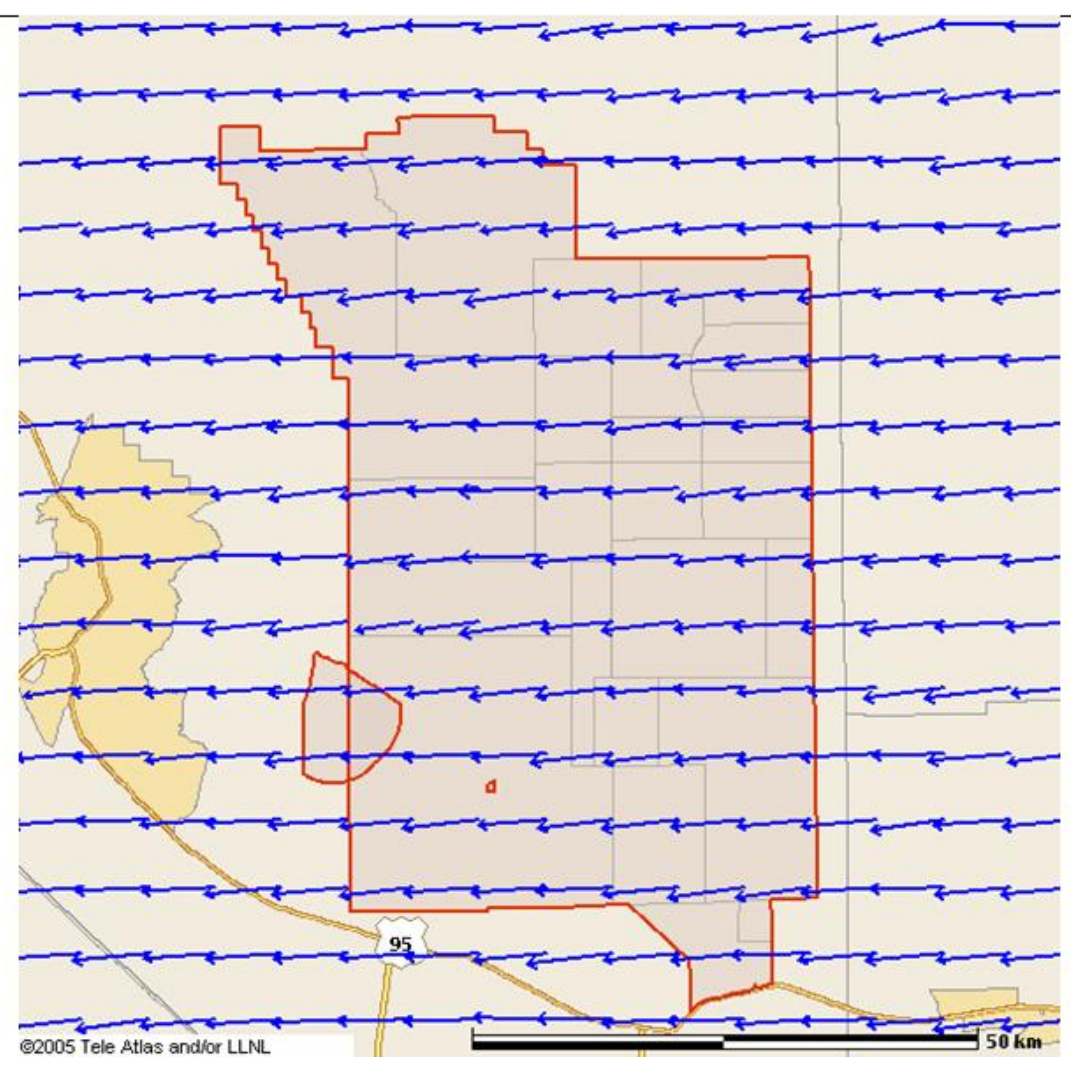




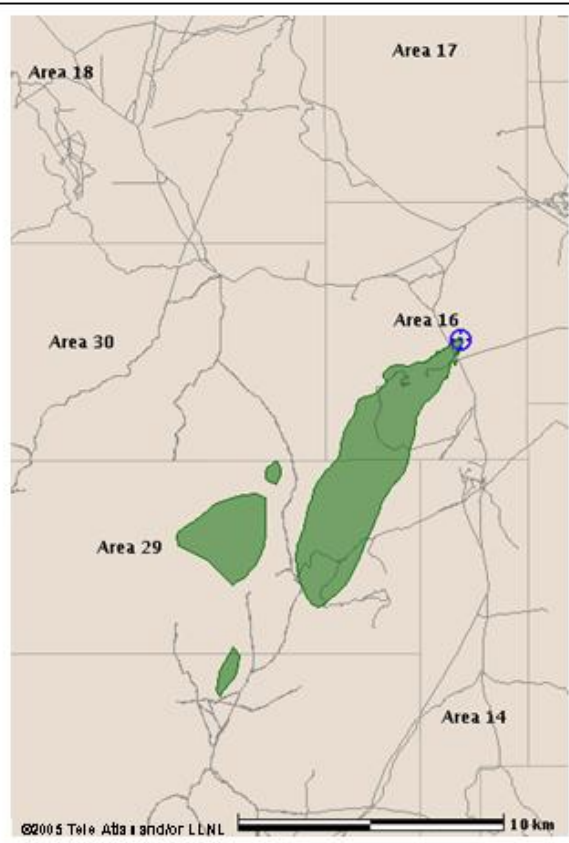

\begin{tabular}{|l|l|l|c|}
\hline \multicolumn{3}{|c|}{ Effects and Actions } \\
\hline & Description & $\begin{array}{l}\text { (mrem) } \\
\text { Extent } \\
\text { Area }\end{array}$ & Population \\
\hline & $10 \%$ of the 0.1 mrem level at which EPA & $>0.01$ & 0 \\
approval is required. & $16.3 \mathrm{~km}$ \\
& $31.7 \mathrm{~km} 2$ & \\
\hline A.reas and counts in the table are cumulative. & \\
\hline
\end{tabular}

Effects $\alpha$ contamination from January $15,200616: 00$ UTC to January 19, $200616: 00$ UTC at $10.0 \mathrm{~m}$.

Release Location: $37.023481 \mathrm{~N}, 116.181963 \mathrm{~W}$

Material: AM-241 + daughters + TH-230 + PU-238 + PA-234M + U-234 + PA-234 +

$\mathrm{RA}-226+\mathrm{PB}-212+\mathrm{PO}-216+\mathrm{BI}-212+\mathrm{TL}-208+\mathrm{PO}-212+\mathrm{RA}-223+\mathrm{FR}-223+\mathrm{AC}-$

$227+\mathrm{TH}-227+\mathrm{PO}-215+\mathrm{RA}-224+\mathrm{TH}-234+\mathrm{PB}-211+\mathrm{Bl}-211+\mathrm{TL}-207+\mathrm{PO}-218+$

$\mathrm{TH}-231+\mathrm{PA}-231+\mathrm{U}-238+\mathrm{CO}-60+\mathrm{TH}-232+\mathrm{RA}-228+\mathrm{AC}-228+\mathrm{PB}-214+\mathrm{Bl}-214+$

$\mathrm{PO}-214+\mathrm{K}-40+\mathrm{PO}-210+\mathrm{PB}-210+\mathrm{Bl}-210+\mathrm{TH}-228+\mathrm{PU}-239+\mathrm{U}-235+\mathrm{Y}-90+$

$\mathrm{SR}-90+\mathrm{CS}-134+\mathrm{CO}-58+\mathrm{BA}-137 \mathrm{M}+\mathrm{CS}-137+\mathrm{H}-3+\mathrm{CM}-243+\mathrm{EU}-154+\mathrm{NB}-94+$

BE-7 + PO-211 + AL-26 + SB-125 + RN-222 + RN-220 + RN-219 + AT-218 + EU-155 +

EU-152

Gener ated On: November 28, 2006 01:14 UTC

Model: ADAPTILODI

Comments: Release starting at 1/15/2006 16:00 UTC

met obs at

1/15/2005 16:00 UTC;1/15/2005 16:15 UTC;1/15/2005 16:30 UTC

1/15/2005 16:45 UTC; 1/15/2005 17:00 UTC; 1/15/2005 17:15 UTC:

1/15/2005 17:30 UTC;1/15/2005 17:45 UTC

Map Size: $31.1 \mathrm{~km}$ by $31.1 \mathrm{~km}$ Id: ProductionT/EVENT 12117Ms 0 porodexec 8inren NARAC Operations: (NARAC Staff); narac@illni.gov; 925-424-6465

Not approved for further distribution 


\section{Jan 20, 2006 - surface winds at release start}

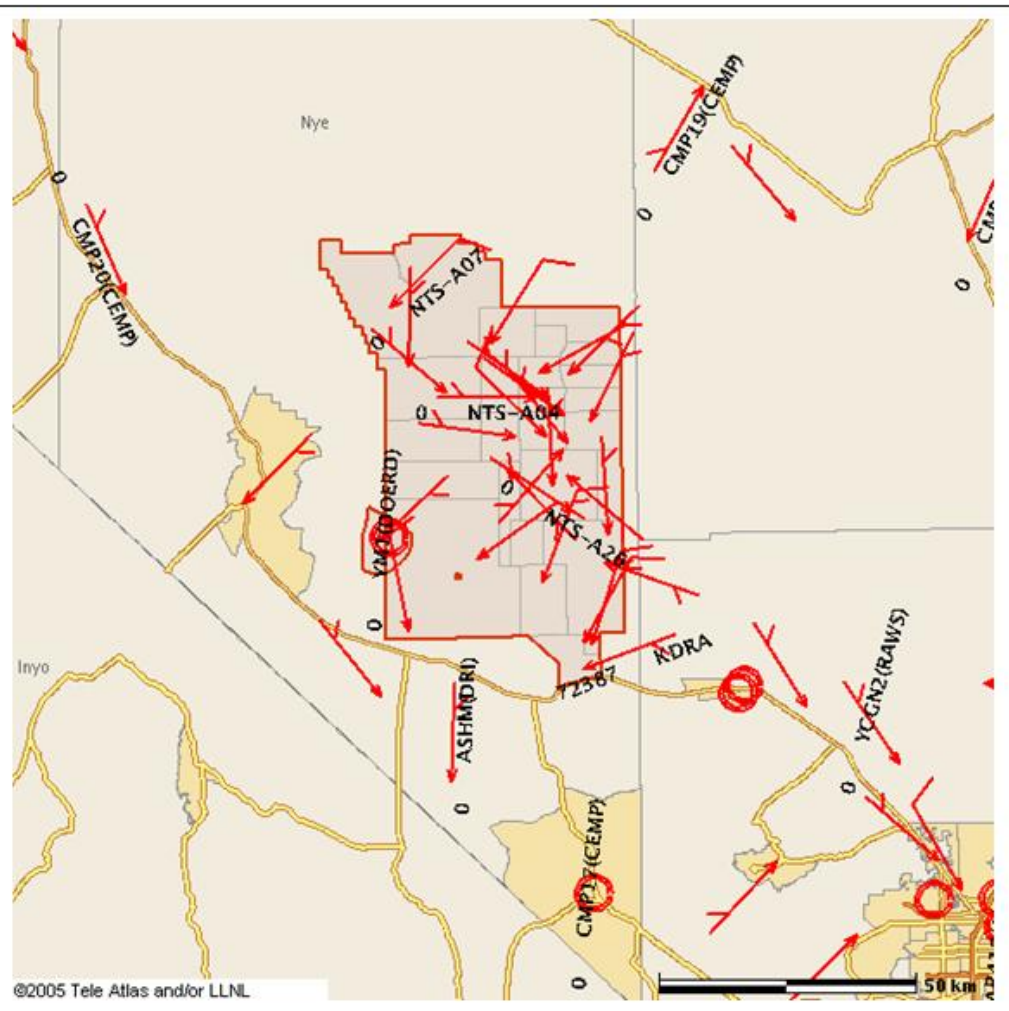




\section{Jan 20, 2006 - midlevel winds at release start}

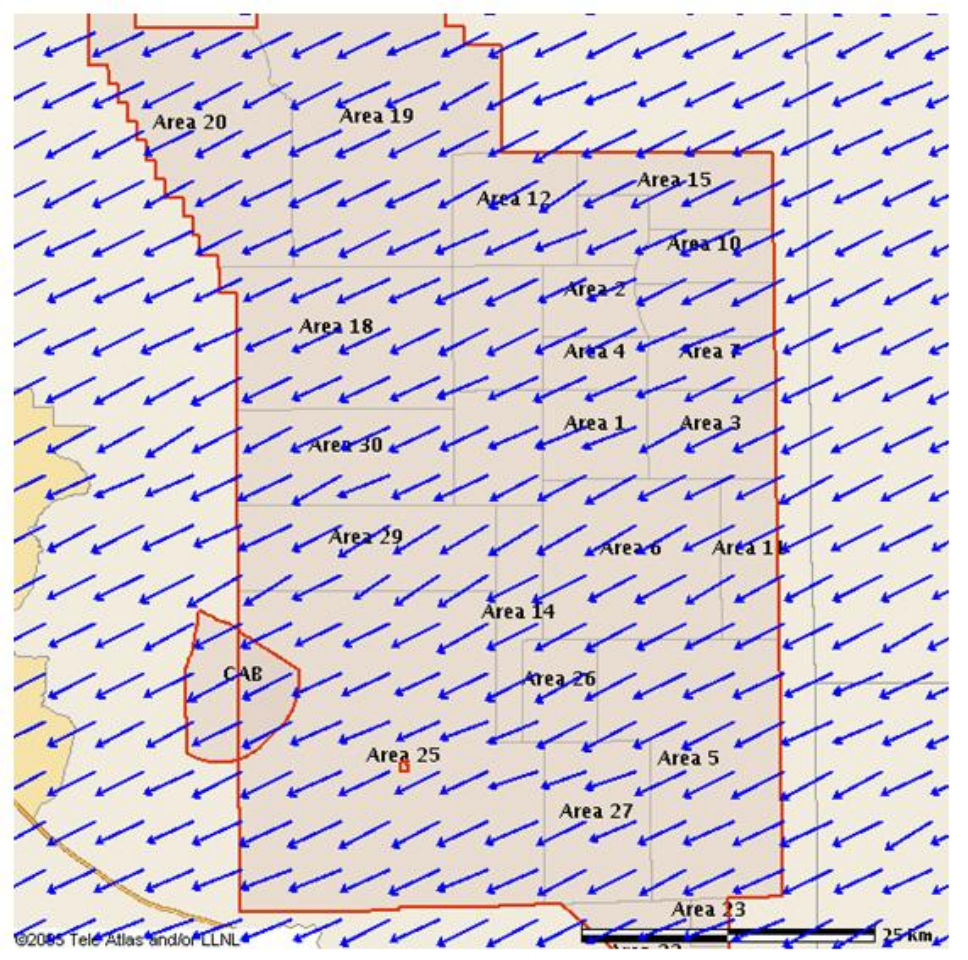


Jan 20, 2006 Example Assessment

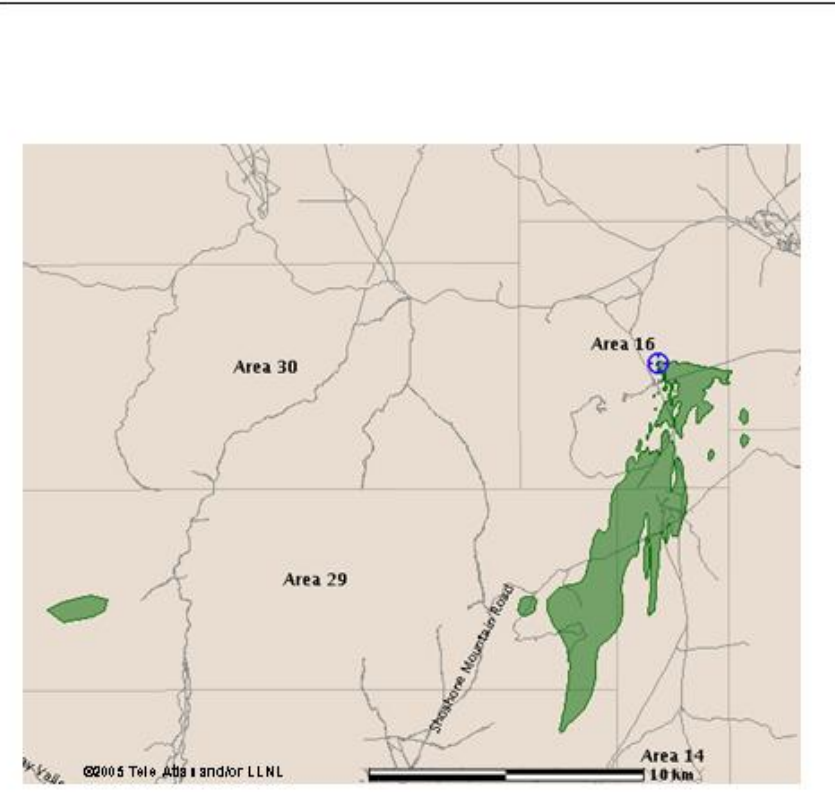

\begin{tabular}{|l|l|l|c|}
\hline \multicolumn{4}{|c|}{ Effects and Actions } \\
\hline & Description & $\begin{array}{l}\text { (mrem) } \\
\text { Extent } \\
\text { Area }\end{array}$ & Population \\
\hline & $\begin{array}{l}10 \% \text { of the } 0.1 \text { mrem level at which EPA } \\
\text { approval is required. }\end{array}$ & $\begin{array}{l}30.01 \\
24.1 \mathrm{~km} \\
24.3 \mathrm{~km} 2\end{array}$ & 0 \\
\hline
\end{tabular}

Effects $\alpha$ contarnination from January 20, 2006 16:00 UTC to January 24, 2006 16:00 UTC at $10.0 \mathrm{~m}$.

Release Location: 37.023481 N $116.181963 \mathrm{~W}$

Material: $\mathrm{AM}-241+$ daughters + $\mathrm{TH}-230+\mathrm{PU}-238+\mathrm{PA}-234 \mathrm{M}+\mathrm{U}-234+\mathrm{PA}-234+$

$\mathrm{RA}_{-2} 26+\mathrm{PB}-212+\mathrm{PO}-216+\mathrm{Bl}-212+\mathrm{TL}-208+\mathrm{PO}-212+\mathrm{RA}-223+\mathrm{FR}-223+\mathrm{AC}-$

$227+\mathrm{TH}-227+\mathrm{PO}-215+\mathrm{RA}-224+\mathrm{TH}-234+\mathrm{PB}-211+\mathrm{Bl}-211+\mathrm{TL}-207+\mathrm{PO}-218+$

$\mathrm{TH}-231+\mathrm{PA}-231+\mathrm{U}-238+\mathrm{CO}-60+\mathrm{TH}-232+\mathrm{RA}-228+\mathrm{AC}-228+\mathrm{PB}-214+\mathrm{Bl}-214+$

$\mathrm{PO}-214+\mathrm{K}-40+\mathrm{PO}-210+\mathrm{PB}-210+\mathrm{Bl}-210+\mathrm{TH}-228+\mathrm{PU}-239+\mathrm{U}-235+\mathrm{Y}-90+$

$\mathrm{SR}-90+\mathrm{CS}-134+\mathrm{CO}-58+\mathrm{BA}-137 \mathrm{M}+\mathrm{CS}-137+\mathrm{H}-3+\mathrm{CM}-243+\mathrm{EU}-154+\mathrm{NB}-94+$

$\mathrm{E}-7+\mathrm{PO}-211+\mathrm{AL}-26+\mathrm{SB}-125+\mathrm{RN}-222+\mathrm{RN}-220+\mathrm{RN}-219+\mathrm{AT}-218+\mathrm{EU}-155+$

EU-152

Cenerded On: November 26, 2006 20:53 UTC

Model: ADAPTRODI

Cormments: Release starting at 1/20/2006 16:00 UTC

met obs at

1/20/2006 16:00 UTC; 1/20/2006 16:15 UTC; 1/20/2006 16:30 UTC

1/20/2006 16:45 UTC;1/20/2006 17:00 UTC; 1/20/2006 17:15 UTC

1/20/2006 17:30 UTC; 1/20/2006 17:45 UTC

Map Size: $29.5 \mathrm{~km}$ by $29.5 \mathrm{~km}$ Id: ProductionT/EVENT_12117/ws_1/prodexec_10inrem NARAC Operations: (NARAC Staff); narac@ilnil.gov; 925-4246465

Not approved for further distribution 


\title{
Appendix C
}

CAP88-PC Version 3.0 Modeling Output for the Proposed Divine Strake Experiment

\author{
June 2006
}

Literature Values (Pre-site Characterization) Used for Source term 
Bechtel Nevada

ENGINEERING DEPARTMENT

ANALYSIS/CALCULATION (A/C) COVER SHEET

Project Title:

Project/Tracking No.:

Analysis/Calculation No.:

NESH-55

Divine Strake

Subject:

Divine Strake Experiment

\begin{tabular}{|l|l|l|l|}
\hline Analysis/Calculation Status Designation: $\square$ Preliminary $\square$ Confirmed & Program No. & Version/Release No. \\
\hline Computer Program/Title & Mainframe/PC & & 3.0 \\
\hline CAP88-PC & PC & & 2.06 \\
\hline Hotspot & PC & & 1.0 \\
\hline Bearing and Distance Program & PC & & \\
\hline
\end{tabular}

Purpose and Objective:

An assessment of the radiation doses that could occur to offsite residents from the dust created by the Divine Strake experiment was conducted to determine whether the experiment would be in conformance with the National Emission Standards for Air Pollutants (NESHAP),

Title 40 of Code of Federal Regulations, Part 61, Subpart H. During the Divine Strake experiment, 700 tons of ammonium nitrate fuel oil

(ANFO) emulsion will be detonated over the U-16b tunnel complex. Conservative assumptions were used for estimating the air emissions

(Enclosure 2) to determine the potential dose with CAP88-PC software and whether EPA approval would be required. If the dose (CEDE) is $\geq$

$0.1 \mathrm{mrem} / \mathrm{yr}$, EPA approval is required.

Since the CAP88-PC model is normally used for chronic continuous radioactive releases, the dose assessment was also made with Hotspot, a model designed by LLNL for explosions and releases occurring over a period less than a few hours.

Summary of Conclusion:

Based upon the radiation doses estimated by the CAP88-PC and Hotspot atmospheric diffusion models, the committed effective dose equivalent (CEDE) to the hypothetical maximally exposed individual (MEI) at the eastern NTS boundary, would be less than $1 \mu$ rem. Other off-site locations are also shown in the figure below. Based on this assessment, approval is not required per 40 CFR 61.96.

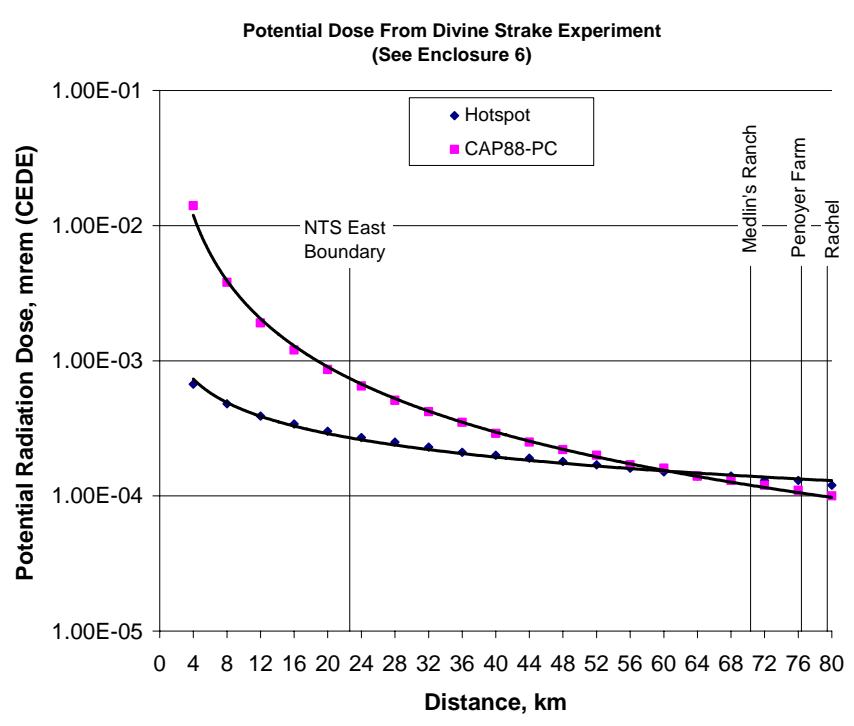

Record of Revisions

\begin{tabular}{|c|c|c|c|c|c|}
\hline $\begin{array}{l}\text { Rev } \\
\text { No. }\end{array}$ & $\begin{array}{c}\text { Reason for } \\
\text { Revision }\end{array}$ & Date & Prepared & Checked & Approval \\
\hline 0 & & $6 / 15 / 2006$ & $\begin{array}{l}\text { Frank } \\
\text { Grossman }\end{array}$ & Ron Warren & Charles Lohrstorfer \\
\hline
\end{tabular}




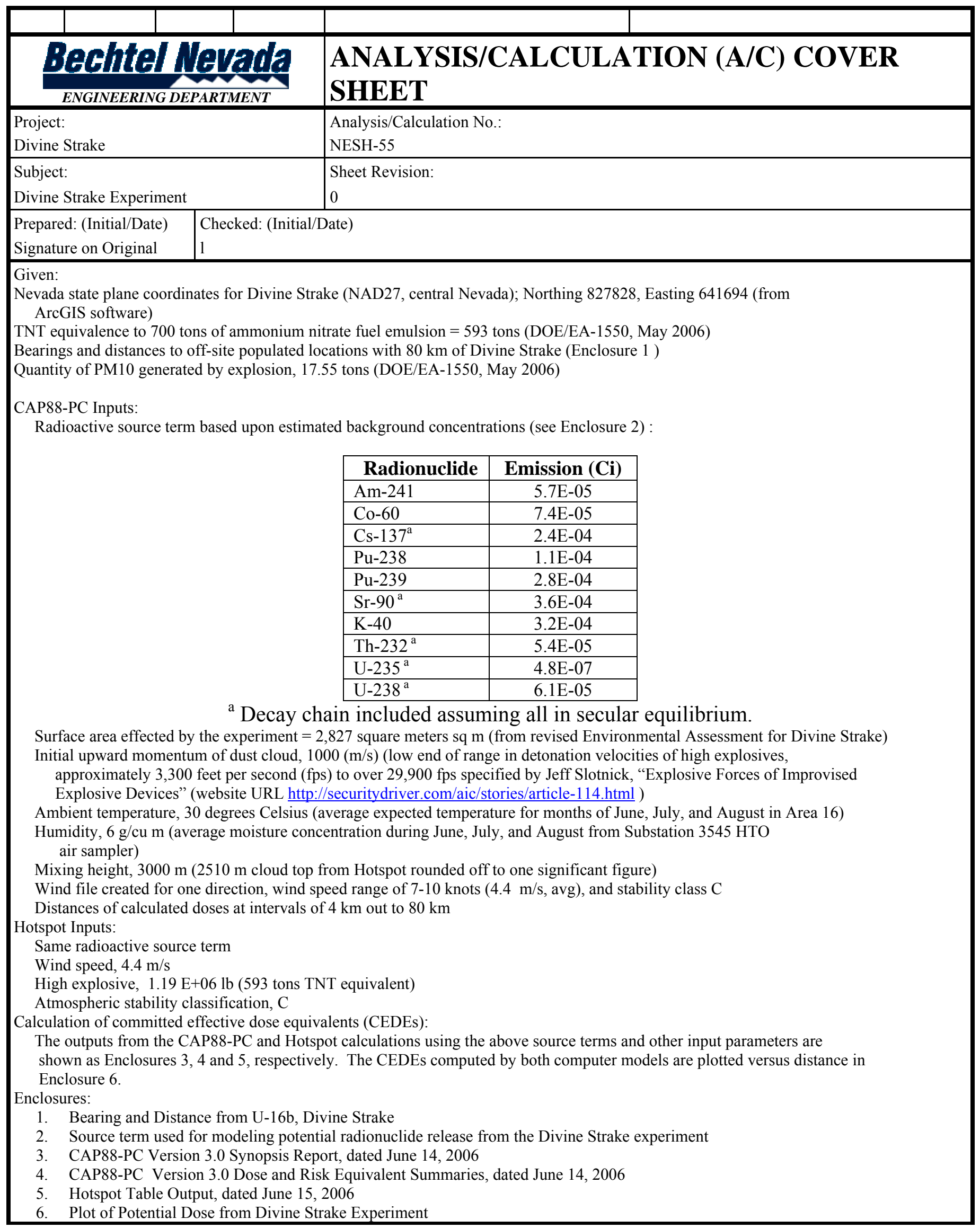




\section{ENCLOSURE 1}

Bearing and Distance from U-16b, Divine Strake, Northing 827828, Easting 641694

Location Sector

NTS East Boundary

Amargosa Valley

springdale

U.S. Ecology

Beatty

Crystal

Amargosa Center

Tolicha Peak

Cactus Springs

Indian springs

Sarcobatus Flat

Medlin's Ranch*

Ash Meadows

Stateline Area

Penoyer Farm*

Cold Creek

SNV Prison

Rachel*
Distance $(\mathrm{km})$

Bearing

22.6

46. 71

51.12

53.27

54.36

58.78

59.11

61.71

63.6

67.53

69.59

70.34

73.52

75.75

76.38

78.44

79.13

79.45
90.00

204.15

271.08

240.21

251.08

179.31

208.56

298.81

139.57

136.92

285.8

52.6

188.03

196.05

22.11

149.26

134.46

27.98
E SSW W

WSW WSW

$\mathrm{S}$

SSW

WNW

SE

SE

WNW

NE

$S$

SSW

NNE

SSE

SE

NNE

*Potentially downwind of Divine Strake 


\section{Enclosure 2}

\section{Source term used for modeling potential radionuclide release from the Divine Strake experiment}

\section{Soil Emissions}

Soil expected to be emitted was expressed in two ways to obtain a radionuclide source term. The first was the PM10 value of 17.55 tons predicted for the Divine Strake experiment and listed in Table 2 of Appendix C of the Environmental Assessment for the Divine Strake Experiment (U.S. Department of Energy, 2006). The second was the surface area of the crater predicted for the Divine Strake experiment ( $30 \mathrm{~m}$ diameter $=2,827 \mathrm{~m}^{2}$ ) (U.S. Department of Energy, 2006). The application of radionuclide concentrations to these values is described below.

\section{Radionuclides}

It was the intention of this assessment to assemble published data on man-made radionuclide concentrations in soil from samples either collected in Area 16 of the NTS or that were analogous to soil in the area of the Divine Strake experiment. Also, concentrations of naturally occurring radionuclides were taken from values listed by the EPA for background levels in soil (U.S. Environmental Protection Agency, 1994). From this compilation of concentrations, maximum values (not including nuclear test locations) were selected for use in modeling potential dose to persons from emissions from the Divine Strake experiment. Radionuclide concentration data and references are listed in Table 1 with shaded emission values being those selected for use in modeling. Radionuclide concentrations expressed as $\mathrm{pCi} / \mathrm{g}$ soil were multiplied by the predicted mass of PM10 to obtain the total potential radionuclide emission. Radionuclide concentrations expressed as $\mathrm{nCi} / \mathrm{m}^{2}$ were multiplied by the predicted surface area of the crater to obtain the total potential radionuclide emission.

In addition to using the highest radionuclide concentrations reported, potential radionuclide emissions from Divine Strake were further maximized by assuming $100 \%$ of the activity associated with soil was emitted to air for transport offsite and no adjustments were made for the physical decay of radionuclides (values taken as reported). This is especially conservative for ${ }^{60} \mathrm{Co}$ with its 5.26 year half-life (data ranging from 1983 to 2002 ) and, to a lesser extent, for ${ }^{137} \mathrm{Cs}$ (30 year half-life) and ${ }^{90} \mathrm{Sr}(27.7$ year half-life).

Three samples of soil and limestone from the Divine Strake excavation were collected on May 24, 2006 by Bechtel Nevada Environmental Technical Services. These samples were screened with a Canberra XtRa coaxial germanium detector (Model GX5520, SN 4945016) to provide some data (Table 2) supporting the assertion that values listed in Table 1 are conservatively high. 
Table 1. Radionuclide emission assumed from the Divine Strake experiment. Shaded emission values were used to model potential dose.

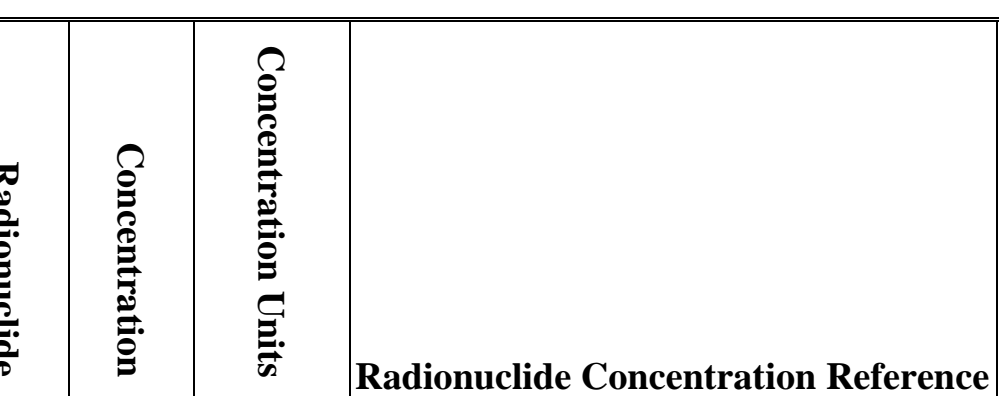

\section{Comments}

background soil around U-16a muckpile

value estimated for Area 16

background soil around U-16a muckpile value estimated for Area 16 contour A in Figure 8

background soil around U-16a muckpile based on measurements along Mid-Valley road from NTS \& global fallout contour $\mathrm{C}$ in Figure 7

\begin{tabular}{|c|c|c|c|c|}
\hline $2827^{\mathrm{b}}$ & $\mathrm{m}^{2}$ & ${ }^{137} \mathrm{Cs}$ & 81 & \begin{tabular}{l|l}
$\mathrm{nCi} / \mathrm{m}^{2}$ & $\mathrm{CDC} \&$ National Cancer Institute, 2005
\end{tabular} \\
\hline
\end{tabular}

\begin{tabular}{|c|c|c|c|c|c|}
\hline $2827^{\mathrm{b}}$ & $\mathrm{m}^{2}$ & ${ }^{137} \mathrm{Cs}$ & 84 & $\mathrm{nCi} / \mathrm{m} 2$ & Bluitt, 1983 \\
\hline
\end{tabular}

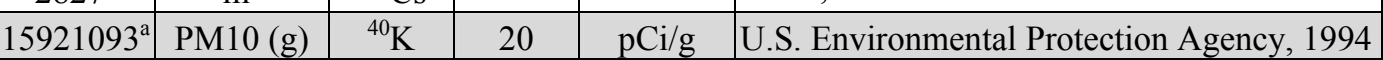

$15921093^{\mathrm{a}}$ PM10 (g) $2827^{\mathrm{b}} \quad \mathrm{m}^{2}$

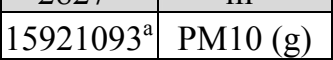
\begin{tabular}{ll}
$\mathrm{Pu}$ & 0.24 \\
\hline
\end{tabular} $2827^{\mathrm{b}}$

\begin{tabular}{|c|c|c|c|}
\hline $15921093^{\mathrm{a}}$ & $\mathrm{PM} 10(\mathrm{~g})$ & ${ }^{90} \mathrm{Sr}$ & 1.17 \\
\hline $2827^{0}$ & & ${ }^{90} \mathrm{Sr}^{\mathrm{c}}$ & 127
\end{tabular}

$$
2827^{\mathrm{b}}
$$$$
2827^{\mathrm{b}}
$$

\begin{tabular}{|c|c}
${ }^{90} \mathrm{Sr}^{\mathrm{c}}$ & 127 \\
\hline${ }^{90} \mathrm{Sr}$ & 27
\end{tabular}
$\mathrm{pCi} / \mathrm{g}$ U.S, Department of Energy, 2006

$\mathrm{nCi} / \mathrm{m}^{2}$ McArthur, 1991

pCi/g U.S, Department of Energy, 2006 $\mathrm{nCi} / \mathrm{m}^{2}$ McArthur, 1991

$\mathrm{nCi} / \mathrm{m}^{2} \quad$ CDC \& National Cancer Institute, 2005

$\mathrm{pCi} / \mathrm{g}$ U.S, Department of Energy, 2006

$\mathrm{nCi} / \mathrm{m}^{2}$ McArthur, 1991

$\mathrm{nCi} / \mathrm{m}^{2} \quad$ CDC \& National Cancer Institute, 2005

$\mathrm{pCi} / \mathrm{g}$

$\mathrm{pCi} / \mathrm{g}$

$\mathrm{pCi} / \mathrm{g}$ U.S.

\begin{tabular}{|l|l|l|l|l|}
\hline $15921093^{\mathrm{a}}$ & PM10 (g) & ${ }^{238} \mathrm{U}^{\mathrm{c}}$ & 3.8 & \\
\hline & & & & \\
\hline
\end{tabular}

Hendricks, et al., 1999

${ }^{a}$ Total PM10 estimated to be released during Divine Strake (17.55 tons) (U.S. Department of Energy, 2006)

${ }^{\mathrm{b}}$ Predicted crater area (U.S. Department of Energy, 2006)

${ }^{\mathrm{c}}$ Decay chain included in estimated emission assuming all in secular equilibrium. 
Table 2. Radionuclides detected in samples collected by BN Environmental Technical Services (May 24, 2006) from material excavated from the pit for the Divine Strake experiment.

\begin{tabular}{|c|c|c|c|c|c|}
\hline Sample No. & Description & $\begin{array}{l}\text { Radio- } \\
\text { nuclide }\end{array}$ & $\begin{array}{l}\text { Result } \\
\text { (pCi/g) }\end{array}$ & $\begin{array}{l}\text { Error (2s) } \\
\text { (pCi/g) }\end{array}$ & $\begin{array}{l}\text { MDC } \\
(\mathrm{pCi} / \mathrm{g})\end{array}$ \\
\hline \multirow{4}{*}{ EM00010380 } & \multirow{4}{*}{$\begin{array}{l}\text { Grab Sample of Soil removed during } \\
\text { excavation of Divine Strake pit }\end{array}$} & ${ }^{40} \mathrm{~K}$ & 14.7 & 2.04 & 1.86 \\
\hline & & ${ }^{212} \mathrm{~Pb}$ & 1.1 & 0.16 & 0.25 \\
\hline & & ${ }^{214} \mathrm{Bi}$ & 1.8 & 0.21 & 0.26 \\
\hline & & ${ }^{214} \mathrm{~Pb}$ & 2.2 & 0.22 & 0.28 \\
\hline \multirow{3}{*}{ EM00010381 } & \multirow{3}{*}{$\begin{array}{c}\text { Grab sample of limestone taken from } \\
\text { material removed from the } \\
\text { excavation of Divine Strake pit }\end{array}$} & ${ }^{40} \mathrm{~K}$ & 7.8 & 1.39 & 1.50 \\
\hline & & ${ }^{212} \mathrm{~Pb}$ & 0.6 & 0.10 & 0.19 \\
\hline & & ${ }^{214} \mathrm{~Pb}$ & 0.8 & 0.14 & 0.24 \\
\hline \multirow{4}{*}{ EM00010381 } & \multirow{4}{*}{$\begin{array}{l}\text { Grab Sample of Soil removed during } \\
\text { excavation of Divine Strake pit }\end{array}$} & ${ }^{40} \mathrm{~K}$ & 11.5 & 1.81 & 1.88 \\
\hline & & ${ }^{212} \mathrm{~Pb}$ & 0.64 & 0.09 & 0.18 \\
\hline & & ${ }^{214} \mathrm{Bi}$ & 1.6 & 0.21 & 0.21 \\
\hline & & ${ }^{214} \mathrm{~Pb}$ & 1.9 & 0.16 & 0.25 \\
\hline
\end{tabular}

\section{References}

Bluitt, C. M., 1986. An Aerial Radiological Survey of Areas 16 and 30 Nevada Test Site. EGG10282-1118, EG\&G Energy Measurements, Inc., Las Vegas, NV.

CDC \& National Cancer Institute, 2005. Report on the Feasibility of a Study of the Health Consequences to the American Population from Nuclear Weapons Tests Conducted by the United States and Other Nations, Volume 1. Department of Health and Human Services, Centers for Disease Control and Prevention (CDC) and the National Cancer Institute, CDC: Atlanta, GA.

Hendricks, T. J., and S. R. Riedhauser, 1999. An Aerial Radiological Survey of the Nevada Test Site. DOE/NV/11718-324. Bechtel Nevada, Las Vegas, NV.

McArthur, R. D., 1991. Radionuclides in Surface Soil at the Nevada Test Site. DOE/NV/10845-02. Water Resources Center Publication 45077, Desert Research Institute, University of Nevada System, Las Vegas, NV.

U.S. Department of Energy, 2006. Large-scale, Open-air Explosive Detonation Divine Strake at the Nevada Test Site, Revised Environmental Assessment. DOE/EA-1550, National Nuclear Security Administration, Nevada Site Office, Las Vegas, NV.

U.S. Environmental Protection Agency, 1994. Technical Support Document for the Development of Radionuclide Cleanup Levels for Soil. EPA 402-R-96-011 A, Office of Radiation and Indoor Air, Washington, DC. 


\title{
ENCLOSURE 3
}

\author{
C A P 88 - P C \\ Version 3.0 \\ Clean Air Act Assessment Package - 1988
}

\begin{abstract}
S Y N N O P S S I S R E P O R T
Non-Radon Individual Assessment

Jun 14, 2006 08:46 pm
\end{abstract}

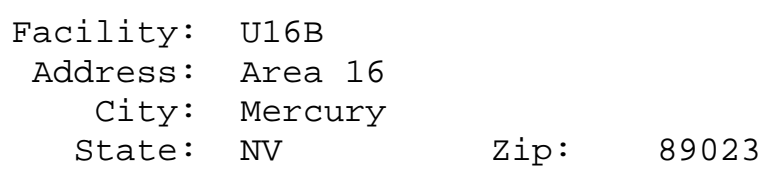

At This Location: 4000 Meters North

Dataset Name: Divine Strake

Dataset Date: 6/14/2006 7:43:00 PM

Wind File: C: \Program Files \CAP88-PC30\Wndfiles \Divine Stake \7-10KNTC.WND 
MAXIMALLY EXPOSED INDIVIDUAL

$\begin{array}{lc}\text { Location of The Individual: } & 4000 \text { Meters North } \\ \text { Lifetime Fatal Cancer Risk: } & 3.90 \mathrm{E}-09\end{array}$

ORGAN DOSE EQUIVALENT SUMMARY

\begin{tabular}{|c|c|}
\hline Organ & $\begin{array}{c}\text { Dose } \\
\text { Equivalent } \\
(\mathrm{mrem} / \mathrm{y})\end{array}$ \\
\hline Adrenals & $5.21 E-04$ \\
\hline B Surfac & $5.75 E-04$ \\
\hline Breasts & $2.06 \mathrm{E}-02$ \\
\hline St Wall & $5.32 \mathrm{E}-\odot 4$ \\
\hline ULI Wall & $5.70 E-\odot 4$ \\
\hline Kidneys & $5.04 \mathrm{E}-04$ \\
\hline Lungs & $5.51 E-\odot 4$ \\
\hline Ovaries & $5.48 \mathrm{E}-04$ \\
\hline $\mathrm{R}$ Marrow & $6.21 \mathrm{E}-04$ \\
\hline Spleen & $8.16 \mathrm{E}-\odot 4$ \\
\hline Thymus & $6.39 E-04$ \\
\hline Uterus & $3.59 E-03$ \\
\hline Bld Wall & $1.86 \mathrm{E}-\odot 3$ \\
\hline Brain & $5.94 \mathrm{E}-\odot 4$ \\
\hline Esophagu & $7.42 \mathrm{E}-\odot 4$ \\
\hline SI Wall & $5.24 \mathrm{E}-\odot 4$ \\
\hline LLI Wall & $2.47 \mathrm{E}-03$ \\
\hline Liver & $6.71 E-03$ \\
\hline Muscle & $5.67 \mathrm{E}-04$ \\
\hline Pancreas & $8.03 E-04$ \\
\hline Skin & $5.36 E-04$ \\
\hline Testes & $5.62 \mathrm{E}-04$ \\
\hline Thyroid & $5.41 E-04$ \\
\hline EFFEC & $1.36 \mathrm{E}-\odot 2$ \\
\hline
\end{tabular}


RADIONUCLIDE EMISSIONS DURING THE YEAR 2006

\begin{tabular}{|c|c|c|c|c|}
\hline Nuclide & Type & Size & $\begin{array}{c}\text { Source } \\
\# 1 \\
\mathrm{Ci} / \mathrm{y}\end{array}$ & $\begin{array}{l}\text { TOTAL } \\
\mathrm{Ci} / \mathrm{y}\end{array}$ \\
\hline Co-60 & M & 1 & $7.4 \mathrm{E}-\odot 5$ & $7.4 \mathrm{E}-05$ \\
\hline Cs - 137 & $\mathrm{~F}$ & 1 & $2.4 \mathrm{E}-\odot 4$ & $2.4 \mathrm{E}-\odot 4$ \\
\hline $\mathrm{Ba}-137 \mathrm{~m}$ & $M$ & 1 & $2.4 \mathrm{E}-\odot 4$ & $2.4 \mathrm{E}-\odot 4$ \\
\hline Am- 241 & M & 1 & $5.7 E-05$ & $5.7 \mathrm{E}-05$ \\
\hline Pu- 238 & $M$ & 1 & $1.1 \mathrm{E}-04$ & $1.1 \mathrm{E}-04$ \\
\hline Pu-239 & $M$ & 1 & $2.8 \mathrm{E}-04$ & $2.8 \mathrm{E}-\odot 4$ \\
\hline Sr-9๑ & M & 1 & $3.6 E-\odot 4$ & $3.6 \mathrm{E}-\odot 4$ \\
\hline Y-9๑ & M & 1 & $3.6 E-04$ & $3.6 E-04$ \\
\hline $\mathrm{K}-40$ & M & 1 & $3.2 \mathrm{E}-\odot 4$ & $3.2 E-04$ \\
\hline Th-232 & $\mathrm{S}$ & 1 & $5.4 \mathrm{E}-05$ & $5.4 \mathrm{E}-05$ \\
\hline $\mathrm{Ra}-228$ & M & 1 & $5.4 \mathrm{E}-05$ & $5.4 \mathrm{E}-\odot 5$ \\
\hline$A C-228$ & $M$ & 1 & $5.4 \mathrm{E}-05$ & $5.4 \mathrm{E}-05$ \\
\hline Th-228 & $\mathrm{s}$ & 1 & $5.4 \mathrm{E}-05$ & $5.4 \mathrm{E}-05$ \\
\hline $\mathrm{Ra}-224$ & M & 1 & $5.4 \mathrm{E}-05$ & $5.4 \mathrm{E}-05$ \\
\hline $\mathrm{Rn}-220$ & G & $\odot$ & $5.4 \mathrm{E}-05$ & $5.4 \mathrm{E}-05$ \\
\hline Po-216 & M & 1 & $5.4 \mathrm{E}-05$ & $5.4 \mathrm{E}-\odot 5$ \\
\hline $\mathrm{Pb}-212$ & M & 1 & $5.4 \mathrm{E}-05$ & $5.4 \mathrm{E}-05$ \\
\hline Bi-212 & M & 1 & $5.4 \mathrm{E}-05$ & $5.4 \mathrm{E}-05$ \\
\hline Po- 212 & M & 1 & $3.5 E-\odot 5$ & $3.5 E-05$ \\
\hline Tl-208 & M & 1 & $1.9 \mathrm{E}-05$ & $1.9 \mathrm{E}-05$ \\
\hline U-235 & M & 1 & $4.8 \mathrm{E}-07$ & $4.8 \mathrm{E}-07$ \\
\hline Th-231 & $\mathrm{S}$ & 1 & $4.8 \mathrm{E}-07$ & $4.8 E-07$ \\
\hline $\mathrm{Pa}-231$ & M & 1 & $4.8 \mathrm{E}-07$ & $4.8 \mathrm{E}-07$ \\
\hline Ac -227 & M & 1 & $4.8 E-07$ & $4.8 \mathrm{E}-07$ \\
\hline Th-227 & $\mathrm{S}$ & 1 & $4.7 \mathrm{E}-07$ & $4.7 \mathrm{E}-07$ \\
\hline$F r-223$ & M & 1 & $6.7 \mathrm{E}-09$ & $6.7 \mathrm{E}-09$ \\
\hline $\mathrm{Ra}-223$ & M & 1 & $4.8 E-07$ & $4.8 E-07$ \\
\hline$R n-219$ & G & $\odot$ & $4.8 \mathrm{E}-07$ & $4.8 \mathrm{E}-07$ \\
\hline Po-215 & $M$ & 1 & $4.8 \mathrm{E}-07$ & $4.8 \mathrm{E}-07$ \\
\hline $\mathrm{Pb}-211$ & M & 1 & $4.8 E-\odot 7$ & $4.8 E-07$ \\
\hline Bi-211 & M & 1 & $4.8 \mathrm{E}-07$ & $4.8 \mathrm{E}-07$ \\
\hline Tl-207 & $M$ & 1 & $4.8 E-07$ & $4.8 \mathrm{E}-07$ \\
\hline Po- 211 & M & 1 & 1. 3E-๑9 & 1. 3E-०9 \\
\hline$U-238$ & M & 1 & $6.1 \mathrm{E}-05$ & $6.1 \mathrm{E}-05$ \\
\hline Th-234 & $\mathrm{s}$ & 1 & $6.1 \mathrm{E}-05$ & $6.1 \mathrm{E}-05$ \\
\hline $\mathrm{Pa}-234 \mathrm{~m}$ & M & 1 & $6.1 \mathrm{E}-05$ & $6.1 \mathrm{E}-05$ \\
\hline $\mathrm{Pa}-234$ & M & 1 & $7.9 \mathrm{E}-\odot 8$ & $7.9 E-08$ \\
\hline$U-234$ & $M$ & 1 & $6.1 \mathrm{E}-05$ & $6.1 \mathrm{E}-05$ \\
\hline Th-230 & $\mathrm{S}$ & 1 & $6.1 \mathrm{E}-05$ & $6.1 \mathrm{E}-05$ \\
\hline $\mathrm{Ra}-226$ & M & 1 & $6.1 \mathrm{E}-05$ & $6.1 \mathrm{E}-05$ \\
\hline Rn- 222 & G & $\odot$ & $6.1 \mathrm{E}-05$ & $6.1 \mathrm{E}-05$ \\
\hline Po- 218 & M & 1 & $6.1 \mathrm{E}-05$ & $6.1 \mathrm{E}-05$ \\
\hline$P b-214$ & $M$ & 1 & $6.1 \mathrm{E}-05$ & $6.1 \mathrm{E}-05$ \\
\hline At -218 & M & 1 & 1. $2 \mathrm{E}-\odot 8$ & 1. $2 \mathrm{E}-\odot 8$ \\
\hline $\mathrm{Bi}-214$ & M & 1 & $6.1 \mathrm{E}-05$ & $6.1 \mathrm{E}-05$ \\
\hline
\end{tabular}




$\begin{array}{lllll}\mathrm{Po}-214 & \mathrm{M} & 1 & 6.1 \mathrm{E}-05 & 6.1 \mathrm{E}-05 \\ \mathrm{~Pb}-210 & \mathrm{M} & 1 & 6.1 \mathrm{E}-05 & 6.1 \mathrm{E}-05 \\ \mathrm{Bi}-210 & \mathrm{M} & 1 & 6.1 \mathrm{E}-05 & 6.1 \mathrm{E}-05 \\ \mathrm{P}-210 & \mathrm{M} & 1 & 6.1 \mathrm{E}-05 & 6.1 \mathrm{E}-05\end{array}$

SITE INFORMATION

Temperature: $\quad 30$ degrees $\mathrm{C}$ Precipitation: $\quad 1 \mathrm{~cm} / \mathrm{y}$

Humidity: $\quad 6 \mathrm{~g} / \mathrm{cu} \mathrm{m}$

Mixing Height: $3000 \mathrm{~m}$ 


\section{SOURCE INFORMATION}

Source Number: 1

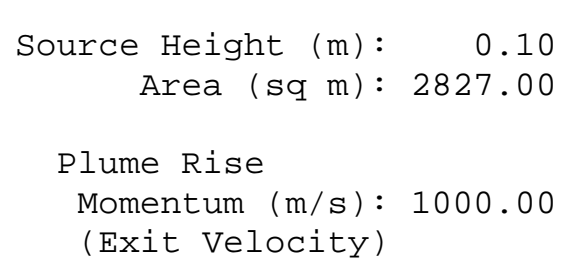

AGRICULTURAL DATA

\begin{tabular}{|c|c|c|c|}
\hline & Vegetable & Milk & Meat \\
\hline Fraction Home Produced: & 0.700 & 0.400 & 0.440 \\
\hline Fraction From Assessment Area: & 0.300 & 0.600 & 0.560 \\
\hline Fraction Imported: & 0.000 & 0.000 & 0.000 \\
\hline
\end{tabular}

Food Arrays were not generated for this run. Default values used.

DISTANCES (M) USED FOR MAXIMUM INDIVIDUAL ASSESSMENT

$\begin{array}{rrrrrrrrrr}4000 & 8000 & 12000 & 16000 & 20000 & 24000 & 28000 & 32000 & 36000 & 40000 \\ 44000 & 48000 & 52000 & 56000 & 60000 & 64000 & 68000 & 72000 & 76000 & 80000\end{array}$




\title{
ENCLOSURE 4
}

\author{
C A P $88-P$ C \\ Version 3.0 \\ Clean Air Act Assessment Package - 1988 \\ D O SE AN D R I S K E Q U IVALEN T S U M MAR I E S \\ Non-Radon Individual Assessment \\ Jun 14, 2006 08:46 pm
}

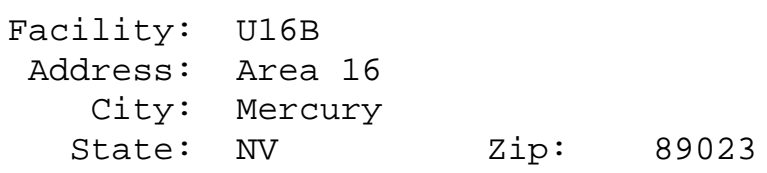

Source Category: Divine Strake Experiment

Source Type: Area

Emission Year: 2006

Comments: Cap88 run to check potential dose

from Divine Strake

Dataset Name: Divine Strake

Dataset Date: 6/14/2006 7:43:00 PM

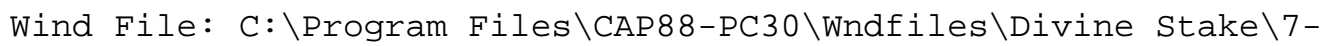
10KNTC.WND 
ORGAN DOSE EQUIVALENT SUMMARY

\begin{tabular}{lc} 
Organ & $\begin{array}{c}\text { Selected } \\
\text { Individual } \\
(\mathrm{mrem} / \mathrm{y})\end{array}$ \\
\cline { 2 - 2 } Adrenals & $5.21 \mathrm{E}-\odot 4$ \\
B Surfac & $5.75 \mathrm{E}-\odot 4$ \\
Breasts & $2.06 \mathrm{E}-\odot 2$ \\
St Wall & $5.32 \mathrm{E}-\odot 4$ \\
ULI Wall & $5.70 \mathrm{E}-\odot 4$ \\
Kidneys & $5.04 \mathrm{E}-\odot 4$ \\
Lungs & $5.51 \mathrm{E}-\odot 4$ \\
Ovaries & $5.48 \mathrm{E}-\odot 4$ \\
R Marrow & $6.21 \mathrm{E}-\odot 4$ \\
Spleen & $8.16 \mathrm{E}-\odot 4$ \\
Thymus & $6.39 \mathrm{E}-\odot 4$ \\
Uterus & $3.59 \mathrm{E}-\odot 3$ \\
Bld Wall & $1.86 \mathrm{E}-\odot 3$ \\
Brain & $5.94 \mathrm{E}-\odot 4$ \\
Esophagu & $7.42 \mathrm{E}-\odot 4$ \\
SI Wall & $5.24 \mathrm{E}-\odot 4$ \\
LLI Wall & $2.47 \mathrm{E}-\odot 3$ \\
Liver & $6.71 \mathrm{E}-\odot 3$ \\
Muscle & $5.67 \mathrm{E}-\odot 4$ \\
Pancreas & $8.03 \mathrm{E}-\odot 4$ \\
Skin & $5.36 \mathrm{E}-\odot 4$ \\
Testes & $5.62 \mathrm{E}-\odot 4$ \\
Thyroid & $5.41 \mathrm{E}-\odot 4$ \\
& $1.36 \mathrm{E}-\odot 2$ \\
EFFEC &
\end{tabular}

PATHWAY EFFECTIVE DOSE EQUIVALENT SUMMARY

Pathway

INGESTION

INHALATION

AIR IMMERSION

GROUND SURFACE

INTERNAL

EXTERNAL

TOTAL
Selected Individual $(\mathrm{mrem} / \mathrm{y})$

$5.87 \mathrm{E}-04$

1. $26 \mathrm{E}-02$

$5.38 \mathrm{E}-08$

$4.96 \mathrm{E}-04$

1. 31E- 02

4. $96 \mathrm{E}-04$

1. 36E- 02 
NUCLIDE EFFECTIVE DOSE EQUIVALENT SUMMARY

\begin{tabular}{|c|c|}
\hline Nuclide & $\begin{array}{c}\text { Selected } \\
\text { Individual } \\
(\text { mrem/y })\end{array}$ \\
\hline Co- 60 & $1.31 \mathrm{E}-04$ \\
\hline Cs -137 & $5.98 \mathrm{E}-05$ \\
\hline $\mathrm{Ba}-137 \mathrm{~m}$ & 1. $01 E-\odot 4$ \\
\hline$A m-241$ & $1.11 \mathrm{E}-03$ \\
\hline$N p-237$ & $\odot . \odot \odot E+\odot \odot$ \\
\hline $\mathrm{Pa}-233$ & $\odot . \odot \odot E+\odot \odot$ \\
\hline U-233 & $\odot . \odot \odot E+\odot \odot$ \\
\hline Th-229 & $\odot . \odot \odot E+\odot \odot$ \\
\hline $\mathrm{Ra}-225$ & $\odot . \odot \odot E+\odot \odot$ \\
\hline$A C-225$ & $\odot . \odot \odot E+\odot \odot$ \\
\hline $\mathrm{Fr}-221$ & $\odot . \odot \odot E+\odot \odot$ \\
\hline At -217 & $\odot . \odot \odot E+\odot \odot$ \\
\hline $\mathrm{Bi}-213$ & $\odot . \odot \odot E+\odot \odot$ \\
\hline Po-213 & $\odot . \odot \odot E+\odot \odot$ \\
\hline$P b-209$ & $\odot . \odot \odot E+\odot \odot$ \\
\hline Tl-209 & $\odot . \odot \odot E+\odot \odot$ \\
\hline Pu-238 & $2.37 \mathrm{E}-03$ \\
\hline U-234 & 1. $01 \mathrm{E}-04$ \\
\hline Th-230 & $4.06 \mathrm{E}-04$ \\
\hline $\mathrm{Ra}-226$ & $1.37 \mathrm{E}-04$ \\
\hline$R n-222$ & 2. $02 \mathrm{E}-12$ \\
\hline Po-218 & $4.06 \mathrm{E}-10$ \\
\hline $\mathrm{Pb}-214$ & $1.17 \mathrm{E}-05$ \\
\hline $\mathrm{Bi}-214$ & $6.80 \mathrm{E}-05$ \\
\hline Po-214 & $3.71 \mathrm{E}-09$ \\
\hline $\mathrm{Pb}-210$ & $7.48 \mathrm{E}-05$ \\
\hline $\mathrm{Bi}-210$ & $4.31 E-06$ \\
\hline Po-210 & $1.07 \mathrm{E}-04$ \\
\hline At -218 & $2.16 \mathrm{E}-15$ \\
\hline Pu-239 & $6.56 \mathrm{E}-03$ \\
\hline U-235 & $7.59 E-07$ \\
\hline Th-231 & $5.84 \mathrm{E}-09$ \\
\hline $\mathrm{Pa}-231$ & $2.10 \mathrm{E}-05$ \\
\hline$A C-227$ & $1.63 E-05$ \\
\hline Th -227 & $2.29 E-06$ \\
\hline $\mathrm{Ra}-223$ & $1.73 E-06$ \\
\hline$R n-219$ & $2.20 \mathrm{E}-12$ \\
\hline Po- 215 & $6.11 \mathrm{E}-11$ \\
\hline $\mathrm{Pb}-211$ & $3.7 \odot E-\odot 8$ \\
\hline $\mathrm{Bi}-211$ & 1. $60 \mathrm{E}-\odot 8$ \\
\hline Tl-207 & $2.02 \mathrm{E}-\odot 8$ \\
\hline Po-211 & $8.68 \mathrm{E}-16$ \\
\hline $\mathrm{Fr}-223$ & $3.22 \mathrm{E}-11$ \\
\hline Sr-9๑ & $1.78 E-\odot 4$ \\
\hline Y-9๑ & $3.06 \mathrm{E}-05$ \\
\hline $\mathrm{K}-40$ & $9.71 \mathrm{E}-05$ \\
\hline Th-232 & $6.30 E-\odot 4$ \\
\hline $\mathrm{Ra}-228$ & $1.52 \mathrm{E}-04$ \\
\hline$A C-228$ & $3.93 E-05$ \\
\hline Th-228 & $9.95 \mathrm{E}-04$ \\
\hline $\mathrm{Ra}-224$ & $7.50 \mathrm{E}-05$ \\
\hline$R n-220$ & $1.73 \mathrm{E}-12$ \\
\hline Po-216 & $6.69 \mathrm{E}-10$ \\
\hline $\mathrm{Pb}-212$ & $9.90 \mathrm{E}-06$ \\
\hline $\mathrm{Bi}-212$ & 1. $01 \mathrm{E}-05$ \\
\hline Po-212 & $\odot . \odot \odot E+\odot \odot$ \\
\hline Tl-208 & $4.44 E-05$ \\
\hline$U-238$ & $8.33 E-05$ \\
\hline Th-234 & $6.57 \mathrm{E}-07$ \\
\hline $\mathrm{Pa}-234 \mathrm{~m}$ & $5.06 \mathrm{E}-\odot 6$ \\
\hline $\mathrm{Pa}-234$ & $2.68 \mathrm{E}-11$ \\
\hline TOTAL & 1. $36 \mathrm{E}-02$ \\
\hline
\end{tabular}


CANCER RISK SUMMARY

\begin{tabular}{lc} 
& $\begin{array}{c}\text { Selected Individual } \\
\text { Total Lifetime } \\
\text { Fatal Cancer Risk }\end{array}$ \\
\cline { 2 - 2 } Esophagu & $1.07 \mathrm{E}-11$ \\
Stomach & $3.56 \mathrm{E}-11$ \\
Colon & $1.22 \mathrm{E}-10$ \\
Liver & $5.91 \mathrm{E}-10$ \\
LUNG & $2.52 \mathrm{E}-09$ \\
Bone & $2.01 \mathrm{E}-10$ \\
Skin & $7.06 \mathrm{E}-12$ \\
Breast & $3.43 \mathrm{E}-11$ \\
Ovary & $4.82 \mathrm{E}-11$ \\
Bladder & $2.55 \mathrm{E}-11$ \\
Kidneys & $1.53 \mathrm{E}-11$ \\
Thyroid & $2.82 \mathrm{E}-12$ \\
Leukemia & $1.48 \mathrm{E}-10$ \\
Residual & $1.32 \mathrm{E}-10$ \\
Total & $3.90 \mathrm{E}-09$ \\
Total & $7.80 \mathrm{E}-09$ \\
&
\end{tabular}

PATHWAY RISK SUMMARY

Pathway

INGESTION

INHALATION

AIR IMMERSION GROUND SURFACE

INTERNAL

EXTERNAL

TOTAL
Selected Individual Total Lifetime Fatal Cancer Risk

2. $63 \mathrm{E}-10$

3. $39 \mathrm{E}-09$

2. $91 \mathrm{E}-14$

$2.46 \mathrm{E}-10$

3. $65 \mathrm{E}-09$

$2.46 \mathrm{E}-10$

3. $90 \mathrm{E}-09$ 
Jun 14, $2006 \quad 08: 46$ pm

NUCLIDE RISK SUMMARY

SUMMARY

Page 4

Selected Individual

Total Lifetime

Nuclide

Fatal Cancer Risk

Co-60

Cs -137

Ba- $137 m$

Am-241

$\mathrm{Np}-237$

$\mathrm{Pa}-233$

$\mathrm{U}-233$

Th -229

$\mathrm{Ra}-225$

Ac -225

$\mathrm{Fr}-221$

At -217

Bi -213

Po -213

$\mathrm{Pb}-209$

Tl-209

$\mathrm{Pu}-238$

$\mathrm{U}-234$

Th -230

Ra-226

Rn-222

Po-218

$\mathrm{Pb}-214$

Bi-214

Po-214

$\mathrm{Pb}-210$

Bi -210

Po-210

At -218

$\mathrm{Pu}-239$

$\mathrm{U}-235$

Th-231

$\mathrm{Pa}-231$

Ac -227

Th- 227

Ra-223

Rn-219

Po- 215

$\mathrm{Pb}-211$

Bi-211

Tl-207

Po-211

$\mathrm{Fr}-223$

$\mathrm{Sr}-90$

$\mathrm{Y}-90$

K-40

Th-232

Ra-228

Ac -228

Th-228

Ra- 224

Rn-220

Po-216

$\mathrm{Pb}-212$

Bi-212

Po- 212

Tl-208

$\mathrm{U}-238$

Th-234

$\mathrm{Pa}-234 \mathrm{~m}$

$7.43 \mathrm{E}-11$

3. $01 \mathrm{E}-11$

5. 43E-11

1. $76 \mathrm{E}-10$

$\odot .00 \mathrm{E}+\odot \odot$

$\odot .00 \mathrm{E}+0 \odot$

$\odot .0 \odot E+\odot \odot$

$\odot .00 \mathrm{E}+\odot \odot$

$\odot .0 \odot \mathrm{E}+\Theta \odot$

$\odot .00 \mathrm{E}+0 \odot$

$\odot .00 \mathrm{E}+0 \odot$

$\odot .00 \mathrm{E}+\odot \odot$

$\odot .00 \mathrm{E}+0 \odot$

$0.00 \mathrm{E}+0 \odot$

$\odot .00 \mathrm{E}+0 \odot$

$\odot .00 \mathrm{E}+0 \odot$

4. $13 \mathrm{E}-10$

8. $28 \mathrm{E}-11$

2. $05 \mathrm{E}-10$

9. 63E-11

1. $10 \mathrm{E}-18$

2. $23 \mathrm{E}-16$

6. $27 \mathrm{E}-12$

3. $61 \mathrm{E}-11$

2. $04 \mathrm{E}-15$

3. $38 \mathrm{E}-11$

2. $53 \mathrm{E}-12$

8. $34 \mathrm{E}-11$

1. $05 \mathrm{E}-21$

1. $04 \mathrm{E}-09$

6. $06 \mathrm{E}-13$

2. $68 \mathrm{E}-15$

1. $99 \mathrm{E}-12$

4. $29 \mathrm{E}-12$

1. $98 \mathrm{E}-12$

1. $47 \mathrm{E}-12$

1. 19E-18

3. $35 \mathrm{E}-17$

1. $36 \mathrm{E}-14$

8. $77 \mathrm{E}-15$

2. $57 \mathrm{E}-15$

4. $75 \mathrm{E}-22$

2. $73 \mathrm{E}-17$

1. $05 \mathrm{E}-10$

3. $87 \mathrm{E}-12$

$6.58 \mathrm{E}-11$

2. $76 \mathrm{E}-10$

6. 53E-11

2. $09 \mathrm{E}-11$

8. $51 \mathrm{E}-10$

6. $44 \mathrm{E}-11$

9. $45 \mathrm{E}-19$

3. $67 \mathrm{E}-16$

6. $75 \mathrm{E}-12$

4. $70 \mathrm{E}-12$

$\odot .00 \mathrm{E}+0 \odot$

2. $42 \mathrm{E}-11$

6. 81E-11

5. 12E-13

8. $11 \mathrm{E}-13$

1. $71 \mathrm{E}-17$

3. $90 \mathrm{E}-\odot 9$

C - 17 
Jun 14, $2006 \quad 08: 46$ pm

SUMMARY

Page 5

INDIVIDUAL EFFECTIVE DOSE EQUIVALENT RATE ( $\mathrm{mrem} / \mathrm{y}$ )

(All Radionuclides and Pathways)

\begin{tabular}{|c|c|c|c|c|c|c|c|}
\hline \multirow[b]{2}{*}{ Direction } & \multicolumn{6}{|c|}{ Distance (m) } & \multirow[b]{2}{*}{$2800 \odot$} \\
\hline & $4 \odot \odot \odot$ & $800 \odot$ & $1200 \odot$ & $1600 \odot$ & $2000 \odot$ & 24000 & \\
\hline $\mathrm{N}$ & 1. $4 \mathrm{E}-02$ & $3.8 \mathrm{E}-03$ & 1. $9 \mathrm{E}-03$ & 1. $2 \mathrm{E}-03$ & $8.6 \mathrm{E}-\odot 4$ & $6.5 \mathrm{E}-\odot 4$ & $5.1 \mathrm{E}-04$ \\
\hline NNW & $\odot . \odot \mathrm{E}+\odot \odot$ & $\odot . \odot E+\odot \odot$ & $\odot . \odot \mathrm{E}+\odot \odot$ & $\odot . \odot E+\odot \odot$ & $\odot . \odot E+\odot \odot$ & $\odot . \odot E+\odot \odot$ & $\odot . \odot E+\odot \odot$ \\
\hline NW & $\odot . \odot \mathrm{E}+\odot \odot$ & $\odot . \odot E+\odot \odot$ & $\odot . \odot \mathrm{E}+\odot \odot$ & $\odot . \odot E+\odot \odot$ & $\odot . \odot E+\odot \odot$ & $\odot . \odot E+\odot \odot$ & $\odot . \odot E+\odot \odot$ \\
\hline WNW & $\odot . \odot \mathrm{E}+\odot \odot$ & $\odot . \odot \mathrm{E}+\odot \odot$ & $\odot . \odot \mathrm{E}+\odot \odot$ & $\odot . \odot \mathrm{E}+\odot \odot$ & $\odot . \odot \mathrm{E}+\odot \odot$ & $\odot . \odot \mathrm{E}+\odot \odot$ & $\odot . \odot \mathrm{E}+\odot \odot$ \\
\hline W & $\odot . \odot \mathrm{E}+\odot \odot$ & $\odot . \odot \mathrm{E}+\odot \odot$ & $\odot . \odot \mathrm{E}+\odot \odot$ & $\odot . \odot E+\odot \odot$ & $\odot . \odot E+\odot \odot$ & $\odot . \odot E+\odot \odot$ & $\odot . \odot E+\odot \odot$ \\
\hline WSW & $\odot . \odot \mathrm{E}+\odot \odot$ & $\odot . \odot \mathrm{E}+\odot \odot$ & $\odot . \odot E+\odot \odot$ & $\odot . \odot E+\odot \odot$ & $\odot . \odot E+\odot \odot$ & $\odot . \odot E+\odot \odot$ & $\odot . \odot E+\odot \odot$ \\
\hline SW & $\odot . \odot \mathrm{E}+\odot \odot$ & $\odot . \odot E+\odot \odot$ & $\odot . \odot E+\odot \odot$ & $\odot . \odot E+\odot \odot$ & $\odot . \odot E+\odot \odot$ & $\odot . \odot E+\odot \odot$ & $\odot . \odot E+\odot \odot$ \\
\hline SSW & $\odot . \odot \mathrm{E}+\odot \odot$ & $\odot . \odot \mathrm{E}+\odot \odot$ & $\odot . \odot \mathrm{E}+\odot \odot$ & $\odot . \odot E+\odot \odot$ & $\odot . \odot E+\odot \odot$ & $\odot . \odot E+\odot \odot$ & $\odot . \odot E+\odot \odot$ \\
\hline S & $\odot . \odot \mathrm{E}+\odot \odot$ & $\odot . \odot \mathrm{E}+\odot \odot$ & $\odot . \odot \mathrm{E}+\odot \odot$ & $\odot . \odot E+\odot \odot$ & $\odot . \odot E+\odot \odot$ & $\odot . \odot E+\odot \odot$ & $\odot . \odot E+\odot \odot$ \\
\hline SSE & $\odot . \odot \mathrm{E}+\odot \odot$ & $\odot . \odot \mathrm{E}+\odot \odot$ & $\odot . \odot \mathrm{E}+\odot \odot$ & $\odot . \odot \mathrm{E}+\odot \odot$ & $\odot . \odot \mathrm{E}+\odot \odot$ & $\odot . \odot E+\odot \odot$ & $\odot . \odot E+\odot \odot$ \\
\hline SE & $\odot . \odot \mathrm{E}+\odot \odot$ & $\odot . \odot \mathrm{E}+\odot \odot$ & $\odot . \odot E+\odot \odot$ & $\odot . \odot E+\odot \odot$ & $\odot . \odot \mathrm{E}+\odot \odot$ & $\odot . \odot E+\odot \odot$ & $\odot . \odot E+\odot \odot$ \\
\hline ESE & $\odot . \odot \mathrm{E}+\odot \odot$ & $\odot . \odot \mathrm{E}+\odot \odot$ & $\odot . \odot \mathrm{E}+\odot \odot$ & $\odot . \odot E+\odot \odot$ & $\odot . \odot E+\odot \odot$ & $\odot . \odot E+\odot \odot$ & $\odot . \odot E+\odot \odot$ \\
\hline$E$ & $\odot . \odot \mathrm{E}+\odot \odot$ & $\odot . \odot \mathrm{E}+\odot \odot$ & $\odot . \odot \mathrm{E}+\odot \odot$ & $\odot . \odot E+\odot \odot$ & $\odot . \odot E+\odot \odot$ & $\odot . \odot E+\odot \odot$ & $\odot . \odot \mathrm{E}+\odot \odot$ \\
\hline ENE & $\odot . \odot \mathrm{E}+\odot \odot$ & $\odot . \odot \mathrm{E}+\odot \odot$ & $\odot . \odot \mathrm{E}+\odot \odot$ & $\odot . \odot E+\odot \odot$ & $\odot . \odot \mathrm{E}+\odot \odot$ & $\odot . \odot E+\odot \odot$ & $\odot . \odot E+\odot \odot$ \\
\hline $\mathrm{NE}$ & $\odot . \odot \mathrm{E}+\odot \odot$ & $\odot . \odot \mathrm{E}+\odot \odot$ & $\odot . \odot E+\odot \odot$ & $\odot . \odot E+\odot \odot$ & $\odot . \odot \mathrm{E}+\odot \odot$ & $\odot . \odot E+\odot \odot$ & $\odot . \odot E+\odot \odot$ \\
\hline NNE & $\odot . \odot \mathrm{E}+\odot \odot$ & $\odot . \odot \mathrm{E}+\odot \odot$ & $\odot . \odot E+\odot \odot$ & $\odot . \odot \mathrm{E}+\odot \odot$ & $\odot . \odot E+\odot \odot$ & $\odot . \odot E+\odot \odot$ & $\odot . \odot \mathrm{E}+\odot \odot$ \\
\hline
\end{tabular}

Distance $(\mathrm{m})$

\begin{tabular}{|c|c|c|c|c|c|c|c|}
\hline Direction & 32000 & 36000 & 40000 & 44000 & 48000 & 52000 & 56000 \\
\hline $\mathrm{N}$ & $4.2 \mathrm{E}-\odot 4$ & $3.5 E-\odot 4$ & $2.9 E-\odot 4$ & $2.5 \mathrm{E}-\odot 4$ & $2.2 \mathrm{E}-\odot 4$ & $2 . \odot E-\odot 4$ & 1. $7 \mathrm{E}-\odot 4$ \\
\hline NNW & $\odot . \odot \mathrm{E}+\odot \odot$ & $\odot . \odot \mathrm{E}+\odot \odot$ & $\odot . \odot E+\odot \odot$ & $\odot . \odot \mathrm{E}+\odot \odot$ & $\odot . \odot \mathrm{E}+\odot \odot$ & $\odot . \odot \mathrm{E}+\odot \odot$ & $\odot . \odot \mathrm{E}+\odot \odot$ \\
\hline NW & $\odot . \odot \mathrm{E}+\odot \odot$ & $\odot . \odot \mathrm{E}+\odot \odot$ & $\odot . \odot E+\odot \odot$ & $\odot . \odot E+\odot \odot$ & $\odot . \odot \mathrm{E}+\odot \odot$ & $\odot . \odot E+\odot \odot$ & $\odot . \odot \mathrm{E}+\odot \odot$ \\
\hline WNW & $\odot . \odot E+\odot \odot$ & $\odot . \odot E+\odot \odot$ & $\odot . \odot E+\odot \odot$ & $\odot . \odot E+\odot \odot$ & $\odot . \odot E+\odot \odot$ & $\odot . \odot E+\odot \odot$ & $\odot . \odot \mathrm{E}+\odot \odot$ \\
\hline W & $\odot . \odot \mathrm{E}+\odot \odot$ & $\odot . \odot \mathrm{E}+\odot \odot$ & $\odot . \odot \mathrm{E}+\odot \odot$ & $\odot . \odot \mathrm{E}+\odot \odot$ & $\odot . \odot \mathrm{E}+\odot \odot$ & $\odot . \odot E+\odot \odot$ & $\odot . \odot \mathrm{E}+\odot \odot$ \\
\hline WSW & $\odot . \odot E+\odot \odot$ & $\odot . \odot E+\odot \odot$ & $\odot . \odot E+\odot \odot$ & $\odot . \odot E+\odot \odot$ & $\odot . \odot E+\odot \odot$ & $\odot . \odot E+\odot \odot$ & $\odot . \odot \mathrm{E}+\odot \odot$ \\
\hline SW & $\odot . \odot E+\odot \odot$ & $\odot . \odot E+\odot \odot$ & $\odot . \odot E+\odot \odot$ & $\odot . \odot E+\odot \odot$ & $\odot . \odot E+\odot \odot$ & $\odot . \odot E+\odot \odot$ & $\odot . \odot \mathrm{E}+\odot \odot$ \\
\hline SSW & $\odot . \odot E+\odot \odot$ & $\odot . \odot E+\odot \odot$ & $\odot . \odot E+\odot \odot$ & $\odot . \odot \mathrm{E}+\odot \odot$ & $\odot . \odot E+\odot \odot$ & $\odot . \odot \mathrm{E}+\odot \odot$ & $\odot . \odot \mathrm{E}+\odot \odot$ \\
\hline$S$ & $\odot . \odot E+\odot \odot$ & $\odot . \odot E+\odot \odot$ & $\odot . \odot E+\odot \odot$ & $\odot . \odot E+\odot \odot$ & $\odot . \odot E+\odot \odot$ & $\odot . \odot E+\odot \odot$ & $\odot . \odot \mathrm{E}+\odot \odot$ \\
\hline SSE & $\odot . \odot E+\odot \odot$ & $\odot . \odot \mathrm{E}+\odot \odot$ & $\odot . \odot E+\odot \odot$ & $\odot . \odot E+\odot \odot$ & $\odot . \odot E+\odot \odot$ & $\odot . \odot \mathrm{E}+\odot \odot$ & $\odot . \odot \mathrm{E}+\odot \odot$ \\
\hline SE & $\odot . \odot \mathrm{E}+\odot \odot$ & $\odot . \odot \mathrm{E}+\odot \odot$ & $\odot . \odot E+\odot \odot$ & $\odot . \odot \mathrm{E}+\odot \odot$ & $\odot . \odot \mathrm{E}+\odot \odot$ & $\odot . \odot \mathrm{E}+\odot \odot$ & $\odot . \odot \mathrm{E}+\odot \odot$ \\
\hline ESE & $\odot . \odot E+\odot \odot$ & $\odot . \odot \mathrm{E}+\odot \odot$ & $\odot . \odot E+\odot \odot$ & $\odot . \odot E+\odot \odot$ & $\odot . \odot E+\odot \odot$ & $\odot . \odot \mathrm{E}+\odot \odot$ & $\odot . \odot \mathrm{E}+\odot \odot$ \\
\hline $\mathrm{E}$ & $\odot . \odot \mathrm{E}+\odot \odot$ & $\odot . \odot \mathrm{E}+\odot \odot$ & $\odot . \odot \mathrm{E}+\odot \odot$ & $\odot . \odot E+\odot \odot$ & $\odot . \odot \mathrm{E}+\odot \odot$ & $\odot . \odot \mathrm{E}+\odot \odot$ & $\odot . \odot \mathrm{E}+\odot \odot$ \\
\hline ENE & $\odot . \odot \mathrm{E}+\odot \odot$ & $\odot . \odot \mathrm{E}+\odot \odot$ & $\odot . \odot E+\odot \odot$ & $\odot . \odot \mathrm{E}+\odot \odot$ & $\odot . \odot \mathrm{E}+\odot \odot$ & $\odot . \odot E+\odot \odot$ & $\odot . \odot \mathrm{E}+\odot \odot$ \\
\hline $\mathrm{NE}$ & $\odot . \odot E+\odot \odot$ & $\odot . \odot \mathrm{E}+\odot \odot$ & $\odot . \odot E+\odot \odot$ & $\odot . \odot E+\odot \odot$ & $\odot . \odot \mathrm{E}+\odot \odot$ & $\odot . \odot E+\odot \odot$ & $\odot . \odot \mathrm{E}+\odot \odot$ \\
\hline NNE & $\odot . \odot E+\odot \odot$ & $\odot . \odot E+\odot \odot$ & $\odot . \odot E+\odot \odot$ & $\odot . \odot \mathrm{E}+\odot \odot$ & $\odot . \odot E+\odot \odot$ & $\odot . \odot \mathrm{E}+\odot \odot$ & $\odot . \odot \mathrm{E}+\odot \odot$ \\
\hline
\end{tabular}


Jun 14, $2006 \quad 08: 46$ pm

SUMMARY

Page 6

INDIVIDUAL EFFECTIVE DOSE EQUIVALENT RATE (mrem/y) (All Radionuclides and Pathways)

\begin{tabular}{|c|c|c|c|c|c|c|}
\hline \multirow[b]{2}{*}{ Direction } & \multicolumn{6}{|c|}{ Distance $(\mathrm{m})$} \\
\hline & 60000 & 64000 & 68000 & 72000 & 76000 & 80000 \\
\hline $\mathrm{N}$ & 1. $6 \mathrm{E}-04$ & 1. $4 \mathrm{E}-04$ & 1. $3 E-04$ & 1. $2 E-04$ & $1.1 E-04$ & 1. $0 \mathrm{E}-\odot 4$ \\
\hline NNW & $\odot . \odot E+\odot \odot$ & $\odot .0 \mathrm{E}+0 \odot$ & $\odot . \odot E+\odot \odot$ & $\odot . \odot E+\odot \odot$ & $\odot .0 \mathrm{E}+\odot \odot$ & $\odot . \odot E+\odot \odot$ \\
\hline NW & $\odot . \odot E+\odot \odot$ & $\odot . \odot E+\odot \odot$ & $\odot . \odot E+\odot \odot$ & $\odot . \odot E+\odot \odot$ & $\odot . \odot E+\odot \odot$ & $\odot . \odot E+\odot \odot$ \\
\hline WNW & $\odot . \odot E+\odot \odot$ & $\odot . \odot E+\odot \odot$ & $\odot . \odot E+\odot \odot$ & $\odot .0 \mathrm{E}+\odot \odot$ & $\odot .0 \mathrm{E}+\odot \odot$ & $\odot . \odot E+\odot \odot$ \\
\hline W & $\odot . \odot E+\odot \odot$ & $\odot .0 \mathrm{E}+0 \odot$ & $\odot . \odot E+\odot \odot$ & $\odot . \odot E+\odot \odot$ & $\odot .0 \mathrm{E}+\odot \odot$ & $\odot . \odot E+\odot \odot$ \\
\hline WSW & $\odot . \odot E+\odot \odot$ & $\odot . \odot E+\odot \odot$ & $\odot . \odot E+\odot \odot$ & $\odot . \odot E+\odot \odot$ & $\odot . \odot E+\odot \odot$ & $\odot . \odot E+\odot \odot$ \\
\hline SW & $\odot . \odot E+\odot \odot$ & $\odot . \odot E+\odot \odot$ & $\odot . \odot E+\odot \odot$ & $\odot . \odot E+\odot \odot$ & $\odot . \odot E+\odot \odot$ & $\odot . \odot E+\odot \odot$ \\
\hline SSW & $\odot . \odot E+\odot \odot$ & $\odot . \odot E+\odot \odot$ & $\odot . \odot E+\odot \odot$ & $\odot .0 \mathrm{E}+\odot \odot$ & $\odot .0 \mathrm{E}+\odot \odot$ & $\odot . \odot E+\odot \odot$ \\
\hline S & $\odot . \odot E+\odot \odot$ & $\odot . \odot E+\odot \odot$ & $\odot . \odot E+\odot \odot$ & $\odot . \odot E+\odot \odot$ & $\odot . \odot E+\odot \odot$ & $\odot . \odot E+\odot \odot$ \\
\hline SSE & $\odot . \odot E+\odot \odot$ & $\odot . \odot E+\odot \odot$ & $\odot . \odot E+\odot \odot$ & $\odot . \odot E+\odot \odot$ & $\odot . \odot \mathrm{E}+\odot \odot$ & $\odot . \odot E+\odot \odot$ \\
\hline SE & $\odot . \odot E+\odot \odot$ & $\odot . \odot E+\odot \odot$ & $\odot . \odot E+\odot \odot$ & $\odot . \odot E+\odot \odot$ & $\odot . \odot \mathrm{O}+\odot \odot$ & $\odot . \odot E+\odot \odot$ \\
\hline ESE & $\odot . \odot E+\odot \odot$ & $\odot .0 \mathrm{E}+0 \odot$ & $\odot . \odot E+\odot \odot$ & $\odot .0 \mathrm{E}+\odot \odot$ & $\odot .0 \mathrm{E}+\odot \odot$ & $\odot . \odot E+\odot \odot$ \\
\hline$E$ & $\odot . \odot E+\odot \odot$ & $\odot . \odot E+\odot \odot$ & $\odot . \odot E+\odot \odot$ & $\odot . \odot E+\odot \odot$ & $\odot .0 \mathrm{E}+\odot \odot$ & $\odot . \odot E+\odot \odot$ \\
\hline ENE & $\odot . \odot E+\odot \odot$ & $\odot . \odot E+\odot \odot$ & $\odot . \odot E+\odot \odot$ & $\odot . \odot E+\odot \odot$ & $\odot . \odot E+\odot \odot$ & $\odot . \odot E+\odot \odot$ \\
\hline NE & $\odot . \odot E+\odot \odot$ & $\odot .0 \mathrm{O}+\odot \odot$ & $\odot . \odot E+\odot \odot$ & $\odot .0 \mathrm{E}+\odot \odot$ & $\odot .0 \mathrm{E}+\odot \odot$ & $\odot . \odot E+\odot \odot$ \\
\hline NNE & $\odot . \odot E+\odot \odot$ & $\odot .0 \mathrm{E}+\odot \odot$ & $\odot . \odot E+\odot \odot$ & $\odot . \odot E+\odot \odot$ & $\odot .0 \mathrm{E}+\odot \odot$ & $\odot . \odot E+\odot \odot$ \\
\hline
\end{tabular}


INDIVIDUAL LIFETIME RISK (deaths)

(All Radionuclides and Pathways)

\begin{tabular}{|c|c|c|c|c|c|c|c|}
\hline \multirow[b]{2}{*}{ Direction } & \multicolumn{6}{|c|}{ Distance (m) } & \multirow[b]{2}{*}{$280 \odot \odot$} \\
\hline & $4 \odot \odot \odot$ & $800 \odot$ & $1200 \odot$ & $1600 \odot$ & $2000 \odot$ & 24000 & \\
\hline $\mathrm{N}$ & $3.9 \mathrm{E}-09$ & 1. $1 \mathrm{E}-09$ & $5.5 \mathrm{E}-10$ & $3.5 \mathrm{E}-10$ & $2.5 \mathrm{E}-10$ & $1.9 \mathrm{E}-10$ & 1. $5 \mathrm{E}-10$ \\
\hline NNW & $\odot . \odot \mathrm{E}+\odot \odot$ & $\odot . \odot \mathrm{E}+\odot \odot$ & $\odot . \odot \mathrm{E}+\odot \odot$ & $\odot . \odot E+\odot \odot$ & $\odot . \odot E+\odot \odot$ & $\odot . \odot E+\odot \odot$ & $\odot . \odot E+\odot \odot$ \\
\hline NW & $\odot . \odot \mathrm{E}+\odot \odot$ & $\odot . \odot E+\odot \odot$ & $\odot . \odot \mathrm{E}+\odot \odot$ & $\odot . \odot E+\odot \odot$ & $\odot . \odot E+\odot \odot$ & $\odot . \odot E+\odot \odot$ & $\odot . \odot E+\odot \odot$ \\
\hline WNW & $\odot . \odot \mathrm{E}+\odot \odot$ & $\odot . \odot \mathrm{E}+\odot \odot$ & $\odot . \odot \mathrm{E}+\odot \odot$ & $\odot . \odot \mathrm{E}+\odot \odot$ & $\odot . \odot \mathrm{E}+\odot \odot$ & $\odot . \odot \mathrm{E}+\odot \odot$ & $\odot . \odot \mathrm{E}+\odot \odot$ \\
\hline W & $\odot . \odot \mathrm{E}+\odot \odot$ & $\odot . \odot \mathrm{E}+\odot \odot$ & $\odot . \odot \mathrm{E}+\odot \odot$ & $\odot . \odot E+\odot \odot$ & $\odot . \odot E+\odot \odot$ & $\odot . \odot E+\odot \odot$ & $\odot . \odot E+\odot \odot$ \\
\hline WSW & $\odot . \odot \mathrm{E}+\odot \odot$ & $\odot . \odot \mathrm{E}+\odot \odot$ & $\odot . \odot \mathrm{E}+\odot \odot$ & $\odot . \odot E+\odot \odot$ & $\odot . \odot E+\odot \odot$ & $\odot . \odot E+\odot \odot$ & $\odot . \odot E+\odot \odot$ \\
\hline SW & $\odot . \odot \mathrm{E}+\odot \odot$ & $\odot . \odot E+\odot \odot$ & $\odot . \odot E+\odot \odot$ & $\odot . \odot E+\odot \odot$ & $\odot . \odot E+\odot \odot$ & $\odot . \odot E+\odot \odot$ & $\odot . \odot E+\odot \odot$ \\
\hline SSW & $\odot . \odot \mathrm{E}+\odot \odot$ & $\odot . \odot \mathrm{E}+\odot \odot$ & $\odot . \odot \mathrm{E}+\odot \odot$ & $\odot . \odot E+\odot \odot$ & $\odot . \odot E+\odot \odot$ & $\odot . \odot E+\odot \odot$ & $\odot . \odot E+\odot \odot$ \\
\hline S & $\odot . \odot \mathrm{E}+\odot \odot$ & $\odot . \odot \mathrm{E}+\odot \odot$ & $\odot . \odot \mathrm{E}+\odot \odot$ & $\odot . \odot E+\odot \odot$ & $\odot . \odot E+\odot \odot$ & $\odot . \odot E+\odot \odot$ & $\odot . \odot E+\odot \odot$ \\
\hline SSE & $\odot . \odot \mathrm{E}+\odot \odot$ & $\odot . \odot \mathrm{E}+\odot \odot$ & $\odot . \odot \mathrm{E}+\odot \odot$ & $\odot . \odot \mathrm{E}+\odot \odot$ & $\odot . \odot \mathrm{E}+\odot \odot$ & $\odot . \odot E+\odot \odot$ & $\odot . \odot E+\odot \odot$ \\
\hline SE & $\odot . \odot \mathrm{E}+\odot \odot$ & $\odot . \odot \mathrm{E}+\odot \odot$ & $\odot . \odot E+\odot \odot$ & $\odot . \odot E+\odot \odot$ & $\odot . \odot \mathrm{E}+\odot \odot$ & $\odot . \odot E+\odot \odot$ & $\odot . \odot E+\odot \odot$ \\
\hline ESE & $\odot . \odot \mathrm{E}+\odot \odot$ & $\odot . \odot \mathrm{E}+\odot \odot$ & $\odot . \odot \mathrm{E}+\odot \odot$ & $\odot . \odot E+\odot \odot$ & $\odot . \odot E+\odot \odot$ & $\odot . \odot E+\odot \odot$ & $\odot . \odot E+\odot \odot$ \\
\hline$E$ & $\odot . \odot \mathrm{E}+\odot \odot$ & $\odot . \odot \mathrm{E}+\odot \odot$ & $\odot . \odot \mathrm{E}+\odot \odot$ & $\odot . \odot E+\odot \odot$ & $\odot . \odot E+\odot \odot$ & $\odot . \odot E+\odot \odot$ & $\odot . \odot \mathrm{E}+\odot \odot$ \\
\hline ENE & $\odot . \odot \mathrm{E}+\odot \odot$ & $\odot . \odot \mathrm{E}+\odot \odot$ & $\odot . \odot \mathrm{E}+\odot \odot$ & $\odot . \odot E+\odot \odot$ & $\odot . \odot \mathrm{E}+\odot \odot$ & $\odot . \odot E+\odot \odot$ & $\odot . \odot E+\odot \odot$ \\
\hline $\mathrm{NE}$ & $\odot . \odot \mathrm{E}+\odot \odot$ & $\odot . \odot \mathrm{E}+\odot \odot$ & $\odot . \odot E+\odot \odot$ & $\odot . \odot E+\odot \odot$ & $\odot . \odot \mathrm{E}+\odot \odot$ & $\odot . \odot E+\odot \odot$ & $\odot . \odot E+\odot \odot$ \\
\hline NNE & $\odot . \odot \mathrm{E}+\odot \odot$ & $\odot . \odot \mathrm{E}+\odot \odot$ & $\odot . \odot E+\odot \odot$ & $\odot . \odot \mathrm{E}+\odot \odot$ & $\odot . \odot E+\odot \odot$ & $\odot . \odot E+\odot \odot$ & $\odot . \odot \mathrm{E}+\odot \odot$ \\
\hline
\end{tabular}

Distance $(\mathrm{m})$

\begin{tabular}{|c|c|c|c|c|c|c|c|}
\hline Direction & 32000 & 36000 & 40000 & 44000 & 48000 & 52000 & 56000 \\
\hline $\mathrm{N}$ & 1. $2 \mathrm{E}-10$ & $1 . \odot E-1 \odot$ & $8.5 E-11$ & 7. 3E-11 & $6.4 \mathrm{E}-11$ & $5.7 \mathrm{E}-11$ & $5.1 \mathrm{E}-11$ \\
\hline NNW & $\odot . \odot \mathrm{E}+\odot \odot$ & $\odot . \odot \mathrm{E}+\odot \odot$ & $\odot . \odot E+\odot \odot$ & $\odot . \odot E+\odot \odot$ & $\odot . \odot \mathrm{E}+\odot \odot$ & $\odot . \odot E+\odot \odot$ & $\odot . \odot E+\odot \odot$ \\
\hline NW & $\odot . \odot \mathrm{E}+\odot \odot$ & $\odot . \odot \mathrm{E}+\odot \odot$ & $\odot . \odot E+\odot \odot$ & $\odot . \odot E+\odot \odot$ & $\odot . \odot \mathrm{E}+\odot \odot$ & $\odot . \odot E+\odot \odot$ & $\odot . \odot E+\odot \odot$ \\
\hline WNW & $\odot . \odot \mathrm{E}+\odot \odot$ & $\odot . \odot \mathrm{E}+\odot \odot$ & $\odot . \odot E+\odot \odot$ & $\odot . \odot E+\odot \odot$ & $\odot . \odot \mathrm{E}+\odot \odot$ & $\odot . \odot E+\odot \odot$ & $\odot . \odot E+\odot \odot$ \\
\hline W & $\odot . \odot \mathrm{E}+\odot \odot$ & $\odot . \odot \mathrm{E}+\odot \odot$ & $\odot . \odot E+\odot \odot$ & $\odot . \odot E+\odot \odot$ & $\odot . \odot \mathrm{E}+\odot \odot$ & $\odot . \odot E+\odot \odot$ & $\odot . \odot E+\odot \odot$ \\
\hline WSW & $\odot . \odot \mathrm{E}+\odot \odot$ & $\odot . \odot \mathrm{E}+\odot \odot$ & $\odot . \odot \mathrm{E}+\odot \odot$ & $\odot . \odot E+\odot \odot$ & $\odot . \odot \mathrm{E}+\odot \odot$ & $\odot . \odot \mathrm{E}+\odot \odot$ & $\odot . \odot \mathrm{E}+\odot \odot$ \\
\hline SW & $\odot . \odot \mathrm{E}+\odot \odot$ & $\odot . \odot \mathrm{E}+\odot \odot$ & $\odot . \odot E+\odot \odot$ & $\odot . \odot E+\odot \odot$ & $\odot . \odot \mathrm{E}+\odot \odot$ & $\odot . \odot E+\odot \odot$ & $\odot . \odot E+\odot \odot$ \\
\hline SSW & $\odot . \odot \mathrm{E}+\odot \odot$ & $\odot . \odot \mathrm{E}+\odot \odot$ & $\odot . \odot \mathrm{E}+\odot \odot$ & $\odot . \odot \mathrm{E}+\odot \odot$ & $\odot . \odot \mathrm{E}+\odot \odot$ & $\odot . \odot E+\odot \odot$ & $\odot . \odot \mathrm{E}+\odot \odot$ \\
\hline $\mathrm{S}$ & $\odot . \odot \mathrm{E}+\odot \odot$ & $\odot . \odot \mathrm{E}+\odot \odot$ & $\odot . \odot \mathrm{E}+\odot \odot$ & $\odot . \odot E+\odot \odot$ & $\odot . \odot \mathrm{E}+\odot \odot$ & $\odot . \odot \mathrm{E}+\odot \odot$ & $\odot . \odot \mathrm{E}+\odot \odot$ \\
\hline SSE & $\odot . \odot \mathrm{E}+\odot \odot$ & $\odot . \odot \mathrm{E}+\odot \odot$ & $\odot . \odot E+\odot \odot$ & $\odot . \odot E+\odot \odot$ & $\odot . \odot \mathrm{E}+\odot \odot$ & $\odot . \odot E+\odot \odot$ & $\odot . \odot E+\odot \odot$ \\
\hline SE & $\odot . \odot \mathrm{E}+\odot \odot$ & $\odot . \odot \mathrm{E}+\odot \odot$ & $\odot . \odot \mathrm{E}+\odot \odot$ & $\odot . \odot E+\odot \odot$ & $\odot . \odot \mathrm{E}+\odot \odot$ & $\odot . \odot E+\odot \odot$ & $\odot . \odot \mathrm{E}+\odot \odot$ \\
\hline ESE & $\odot . \odot \mathrm{E}+\odot \odot$ & $\odot . \odot \mathrm{E}+\odot \odot$ & $\odot . \odot \mathrm{E}+\odot \odot$ & $\odot . \odot \mathrm{E}+\odot \odot$ & $\odot . \odot \mathrm{E}+\odot \odot$ & $\odot . \odot \mathrm{E}+\odot \odot$ & $\odot . \odot E+\odot \odot$ \\
\hline $\mathrm{E}$ & $\odot . \odot \mathrm{E}+\odot \odot$ & $\odot . \odot \mathrm{E}+\odot \odot$ & $\odot . \odot \mathrm{E}+\odot \odot$ & $\odot . \odot E+\odot \odot$ & $\odot .0 \mathrm{E}+\odot \odot$ & $\odot . \odot \mathrm{E}+\odot \odot$ & $\odot . \odot E+\odot \odot$ \\
\hline ENE & $\odot . \odot \mathrm{E}+\odot \odot$ & $\odot . \odot \mathrm{E}+\odot \odot$ & $\odot . \odot E+\odot \odot$ & $\odot . \odot \mathrm{E}+\odot \odot$ & $\odot . \odot E+\odot \odot$ & $\odot . \odot E+\odot \odot$ & $\odot . \odot E+\odot \odot$ \\
\hline $\mathrm{NE}$ & $\odot . \odot \mathrm{E}+\odot \odot$ & $\odot . \odot \mathrm{E}+\odot \odot$ & $\odot . \odot \mathrm{E}+\odot \odot$ & $\odot . \odot E+\odot \odot$ & $\odot . \odot \mathrm{E}+\odot \odot$ & $\odot . \odot \mathrm{E}+\odot \odot$ & $\odot . \odot \mathrm{E}+\odot \odot$ \\
\hline NNE & $\odot . \odot E+\odot \odot$ & $\odot . \odot E+\odot \odot$ & $\odot . \odot E+\odot \odot$ & $\odot . \odot \mathrm{E}+\odot \odot$ & $\odot . \odot \mathrm{E}+\odot \odot$ & $\odot . \odot \mathrm{E}+\odot \odot$ & $\odot . \odot E+\odot \odot$ \\
\hline
\end{tabular}


Jun 14, $2006 \quad 08: 46$ pm

SUMMARY

Page 8

INDIVIDUAL LIFETIME RISK (deaths)

(All Radionuclides and Pathways)

\begin{tabular}{|c|c|c|c|c|c|c|}
\hline \multirow[b]{2}{*}{ Direction } & \multicolumn{6}{|c|}{ Distance (m) } \\
\hline & 60000 & $640 \odot \odot$ & 68000 & $7200 \odot$ & $7600 \odot$ & $800 \odot \odot$ \\
\hline $\mathrm{N}$ & $4.5 \mathrm{E}-11$ & $4.1 E-11$ & $3.8 \mathrm{E}-11$ & $3.4 \mathrm{E}-11$ & $3.2 E-11$ & $2.9 \mathrm{E}-11$ \\
\hline NNW & $\odot . \odot \mathrm{E}+\odot \odot$ & $\odot . \odot E+\odot \odot$ & $\odot . \odot E+\odot \odot$ & $\odot . \odot \mathrm{Q}+\odot \odot$ & $\odot . \odot E+\odot \odot$ & $\odot . \odot E+\odot \odot$ \\
\hline NW & $\odot . \odot \mathrm{E}+\odot \odot$ & $\odot . \odot E+\odot \odot$ & $\odot . \odot \mathrm{E}+\odot \odot$ & $\odot . \odot \mathrm{O}+\odot \odot$ & $\odot . \odot \mathrm{O}+\odot \odot$ & $\odot . \odot E+\odot \odot$ \\
\hline WNW & $\odot . \odot E+\odot \odot$ & $\odot . \odot E+\odot \odot$ & $\odot . \odot \mathrm{E}+\odot \odot$ & $\odot . \odot E+\odot \odot$ & $\odot . \odot \mathrm{O}+\odot \odot$ & $\odot . \odot E+\odot \odot$ \\
\hline W & $\odot . \odot E+\odot \odot$ & $\odot . \odot \mathrm{E}+\odot \odot$ & $\odot . \odot E+\odot \odot$ & $\odot . \odot E+\odot \odot$ & $\odot . \odot \mathrm{O}+\odot \odot$ & $\odot . \odot E+\odot \odot$ \\
\hline WSW & $\odot . \odot \mathrm{E}+\odot \odot$ & $\odot . \odot E+\odot \odot$ & $\odot . \odot \mathrm{E}+\odot \odot$ & $\odot . \odot \mathrm{O}+\odot \odot$ & $\odot . \odot \mathrm{O}+\odot \odot$ & $\odot . \odot E+\odot \odot$ \\
\hline SW & $\odot . \odot E+\odot \odot$ & $\odot . \odot E+\odot \odot$ & $\odot . \odot E+\odot \odot$ & $\odot . \odot E+\odot \odot$ & $\odot .0 \mathrm{E}+\odot \odot$ & $\odot . \odot E+\odot \odot$ \\
\hline SSW & $\odot . \odot \mathrm{OE}+\odot \odot$ & $\odot . \odot E+\odot \odot$ & $\odot . \odot \mathrm{E}+\odot \odot$ & $\odot . \odot E+\odot \odot$ & $\odot . \odot \mathrm{O}+\odot \odot$ & $\odot . \odot E+\odot \odot$ \\
\hline S & $\odot . \odot E+\odot \odot$ & $\odot .0 \mathrm{E}+0 \odot$ & $\odot . \odot E+\odot \odot$ & $\odot . \odot E+\odot \odot$ & $\odot .0 \mathrm{E}+\odot \odot$ & $\odot . \odot E+\odot \odot$ \\
\hline SSE & $\odot . \odot \mathrm{E}+\odot \odot$ & $\odot . \odot E+\odot \odot$ & $\odot . \odot \mathrm{E}+\odot \odot$ & $\odot . \odot \mathrm{O}+\odot \odot$ & $\odot . \odot \mathrm{O}+\odot \odot$ & $\odot . \odot E+\odot \odot$ \\
\hline SE & $\odot . \odot E+\odot \odot$ & $\odot . \odot E+\odot \odot$ & $\odot . \odot E+\odot \odot$ & $\odot . \odot E+\odot \odot$ & $\odot .0 \mathrm{E}+\odot \odot$ & $\odot . \odot E+\odot \odot$ \\
\hline ESE & $\odot . \odot E+\odot \odot$ & $\odot . \odot E+\odot \odot$ & $\odot . \odot E+\odot \odot$ & $\odot . \odot \mathrm{O}+\odot \odot$ & $\odot . \odot \mathrm{OE}+\odot \odot$ & $\odot . \odot E+\odot \odot$ \\
\hline$E$ & $\odot . \odot E+\odot \odot$ & $\odot .0 \mathrm{E}+0 \odot$ & $\odot . \odot E+\odot \odot$ & $\odot . \odot E+\odot \odot$ & $\odot . \odot E+\odot \odot$ & $\odot . \odot E+\odot \odot$ \\
\hline ENE & $\odot . \odot \mathrm{E}+\odot \odot$ & $\odot . \odot E+\odot \odot$ & $\odot . \odot \mathrm{E}+\odot \odot$ & $\odot . \odot \mathrm{E}+\odot \odot$ & $\odot . \odot \mathrm{E}+\odot \odot$ & $\odot . \odot E+\odot \odot$ \\
\hline NE & $\odot . \odot E+\odot \odot$ & $\odot . \odot E+\odot \odot$ & $\odot . \odot E+\odot \odot$ & $\odot . \odot E+\odot \odot$ & $\odot . \odot E+\odot \odot$ & $\odot . \odot E+\odot \odot$ \\
\hline NNE & $\odot . \odot E+\odot \odot$ & $\odot . \odot \mathrm{O}+\odot \odot$ & $\odot . \odot \mathrm{O}+\odot \odot$ & $\odot . \odot \mathrm{O}+\odot \odot$ & $\odot .0 \mathrm{E}+\odot \odot$ & $\odot . \odot E+\odot \odot$ \\
\hline
\end{tabular}




\author{
C A P 88 - P C \\ Version 3.0 \\ Clean Air Act Assessment Package - 1988 \\ WEATHER D A T A \\ Non-Radon Individual Assessment \\ Jun 14, 2006 08:46 pm
}

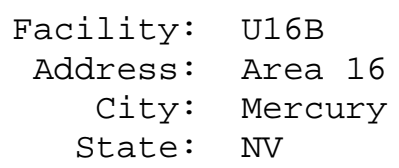

Comments: Cap88 run to check potential dose from Divine Strake

Dataset Name: Divine Strake

Dataset Date: 6/14/2006 7:43:00 PM

Wind File: C: \Program Files \CAP88-PC30\Wndfiles \Divine Stake \710KNTC. WND 
HARMONIC AVERAGE WIND SPEEDS (WIND TOWARDS)

\begin{tabular}{|c|c|c|c|c|c|c|c|c|}
\hline \multirow[b]{2}{*}{ Dir } & \multicolumn{7}{|c|}{ Pasquill Stability Class } & \multirow[b]{2}{*}{$\begin{array}{l}\text { Wind } \\
\text { Freq }\end{array}$} \\
\hline & $A$ & B & C & $\mathrm{D}$ & $E$ & $F$ & G & \\
\hline $\mathrm{N}$ & $\odot .0 \odot \odot$ & 0.000 & 4.373 & 0.000 & 0.000 & 0.000 & $\odot .00 \odot$ & 1.000 \\
\hline NNW & 0.000 & 0.000 & 0.000 & 0.000 & 0.000 & 0.000 & 0.000 & 0.000 \\
\hline NW & $\odot .000$ & $\odot .000$ & $\odot .0 \odot \odot$ & 0.000 & $\odot .000$ & $\odot .000$ & $\odot .0 \odot \odot$ & $\odot .000$ \\
\hline WNW & 0.000 & 0.000 & 0.000 & 0.000 & 0.000 & 0.000 & 0.000 & 0.000 \\
\hline W & $\odot .0 \odot \odot$ & $\odot .00 \odot$ & 0.000 & 0.000 & $\odot .000$ & $\odot .000$ & 0.000 & 0.000 \\
\hline WSW & $\odot .000$ & $\odot .000$ & $\odot .000$ & 0.000 & $\odot .000$ & 0.000 & $\odot .000$ & $\odot .000$ \\
\hline SW & $\odot .000$ & $\odot .000$ & $\odot .0 \odot \odot$ & 0.000 & $\odot .000$ & $\odot .000$ & $\odot .0 \odot \odot$ & $\odot .000$ \\
\hline SSW & $\odot .000$ & $\odot .000$ & $\odot .0 \odot \odot$ & 0.000 & $\odot .000$ & $\odot .000$ & $\odot .0 \odot \odot$ & $\odot .000$ \\
\hline$S$ & $\odot .0 \odot \odot$ & $\odot .00 \odot$ & $\odot .00 \odot$ & $\odot .00 \odot$ & $\odot .000$ & $\odot .0 \odot \odot$ & $\odot .0 \odot \odot$ & $\odot .00 \odot$ \\
\hline SSE & 0.000 & $\odot .000$ & $\odot .0 \odot \odot$ & 0.000 & $\odot .000$ & $\odot .000$ & $\odot .0 \odot \odot$ & $\odot .000$ \\
\hline SE & $\odot .000$ & $\odot .000$ & $\odot .0 \odot \odot$ & 0.000 & $\odot .000$ & $\odot .000$ & $\odot .0 \odot \odot$ & $\odot .00 \odot$ \\
\hline ESE & 0.000 & 0.000 & 0.000 & $\odot .000$ & 0.000 & 0.000 & $\odot .000$ & $\odot .000$ \\
\hline$E$ & $\odot .0 \odot \odot$ & $\odot .00 \odot$ & $\odot .0 \odot \odot$ & $\odot .0 \odot \odot$ & $\odot .000$ & $\odot .000$ & 0.000 & 0.000 \\
\hline ENE & 0.000 & $\odot .000$ & $\odot .0 \odot \odot$ & $\odot .000$ & $\odot .000$ & $\odot .000$ & $\odot .0 \odot \odot$ & $\odot .000$ \\
\hline NE & 0.000 & 0.000 & 0.000 & 0.000 & 0.000 & 0.000 & $\odot .000$ & 0.000 \\
\hline NNE & 0.000 & 0.000 & 0.000 & $\odot .000$ & 0.000 & 0.000 & $\odot .000$ & 0.000 \\
\hline
\end{tabular}

ARITHMETIC AVERAGE WIND SPEEDS (WIND TOWARDS)

Pasquill Stability Class

\begin{tabular}{|c|c|c|c|c|c|c|c|}
\hline Dir & $A$ & B & C & $D$ & $E$ & $\mathrm{~F}$ & G \\
\hline $\mathrm{N}$ & $\odot .0 \odot \odot$ & 0.000 & 4.373 & 0.000 & 0.000 & 0.000 & 0.000 \\
\hline NNW & 0.000 & 0.000 & 0.000 & 0.000 & 0.000 & 0.000 & 0.000 \\
\hline NW & 0.000 & $\odot .000$ & 0.000 & 0.000 & 0.000 & 0.000 & 0.000 \\
\hline WNW & $\odot .0 \odot \odot$ & 0.000 & $\odot .0 \odot \odot$ & $\odot .000$ & $\odot .000$ & $\odot .000$ & 0.000 \\
\hline W & $\odot .00 \odot$ & 0.000 & $\odot .00 \odot$ & 0.000 & $\odot .000$ & $\odot .0 \odot \odot$ & $\odot .0 \odot \odot$ \\
\hline WSW & 0.000 & $\odot .000$ & 0.000 & 0.000 & 0.000 & 0.000 & 0.000 \\
\hline SW & 0.000 & 0.000 & 0.000 & 0.000 & 0.000 & 0.000 & 0.000 \\
\hline SSW & $\odot . \odot \odot \odot$ & 0.000 & $\odot .00 \odot$ & $\odot .0 \odot \odot$ & $\odot .000$ & $\odot .0 \odot \odot$ & $\odot .000$ \\
\hline S & 0.000 & 0.000 & 0.000 & 0.000 & 0.000 & 0.000 & 0.000 \\
\hline SSE & 0.000 & $\odot .000$ & 0.000 & 0.000 & 0.000 & 0.000 & 0.000 \\
\hline SE & 0.000 & 0.000 & 0.000 & 0.000 & 0.000 & 0.000 & 0.000 \\
\hline ESE & $0.00 \odot$ & 0.000 & 0.000 & 0.000 & 0.000 & 0.000 & 0.000 \\
\hline$E$ & 0.000 & 0.000 & 0.000 & 0.000 & 0.000 & 0.000 & 0.000 \\
\hline ENE & 0.000 & $\odot .000$ & 0.000 & 0.000 & 0.000 & 0.000 & 0.000 \\
\hline NE & 0.000 & 0.000 & 0.000 & 0.000 & 0.000 & 0.000 & 0.000 \\
\hline NNE & $\odot .00 \odot$ & 0.000 & 0.000 & $\odot .000$ & 0.000 & 0.000 & $\odot .000$ \\
\hline
\end{tabular}


FREQUENCIES OF STABILITY CLASSES (WIND TOWARDS)

\begin{tabular}{|c|c|c|c|c|c|c|c|}
\hline \multirow[b]{2}{*}{ Dir } & \multicolumn{7}{|c|}{ Pasquill Stability Class } \\
\hline & A & B & C & D & E & $\mathrm{F}$ & G \\
\hline $\mathrm{N}$ & $\odot . \odot \odot \odot \odot$ & $\odot . \odot \odot \odot \odot$ & $1.000 \odot$ & $\odot . \odot \odot \odot \odot$ & $\odot . \odot \odot \odot \odot$ & $\odot . \odot \odot \odot \odot$ & 0.0000 \\
\hline NNW & $\odot .0 \odot \odot \odot$ & $\odot . \odot \odot \odot \odot$ & $\odot .0 \odot \odot \odot$ & $\odot . \odot \odot \odot \odot$ & $\odot . \odot \odot \odot \odot$ & $\odot .0 \odot \odot \odot$ & $\odot .000 \odot$ \\
\hline NW & $\odot . \odot \odot \odot \odot$ & $\odot . \odot \odot \odot \odot$ & $\odot . \odot \odot \odot \odot$ & $\odot . ๑ \odot \odot \odot$ & $\odot . \odot \odot \odot \odot$ & $\odot . \odot \odot \odot \odot$ & $\odot .0 \odot \odot \odot$ \\
\hline WNW & $\odot . \odot \odot \odot \odot$ & $\odot . \odot \odot \odot \odot$ & $\odot . \odot \odot \odot \odot$ & 0.0000 & 0.0000 & 0.0000 & 0.0000 \\
\hline W & $\odot .0 \odot \odot \odot$ & $\odot . \odot \odot \odot \odot$ & $\odot . \odot \odot \odot \odot$ & $\odot . \odot \odot \odot \odot$ & $\odot .0 \odot \odot \odot$ & $0.000 \odot$ & $\odot .0000$ \\
\hline WSW & $\odot . \odot \odot \odot \odot$ & $\odot . \odot \odot \odot \odot$ & $\odot . \odot \odot \odot \odot$ & $\odot . ๑ \odot \odot \odot$ & $\odot . \odot \odot \odot \odot$ & $\odot . \odot \odot \odot \odot$ & $\odot .0 \odot \odot \odot$ \\
\hline SW & $\odot .0 \odot \odot \odot$ & $\odot .0 \odot \odot \odot$ & $\odot . \odot \odot \odot \odot$ & $\odot .0 \odot \odot \odot$ & $\odot .0 \odot \odot \odot$ & $\odot .0 \odot \odot \odot$ & 0.0000 \\
\hline SSW & $\odot . \odot \odot \odot \odot$ & $\odot .0 \odot \odot \odot$ & $\odot .0 \odot \odot \odot$ & $\odot . \odot \odot \odot \odot$ & $\odot . \odot \odot \odot \odot$ & $\odot .0 \odot \odot \odot$ & $\odot .000 \odot$ \\
\hline S & $\odot . \odot \odot \odot \odot$ & $\odot . \odot \odot \odot \odot$ & $\odot . \odot \odot \odot \odot$ & $\odot . \odot \odot \odot \odot$ & $\odot . \odot \odot \odot \odot$ & $\odot . \odot \odot \odot \odot$ & $\odot .0 \odot \odot \odot$ \\
\hline SSE & 0.0000 & 0.0000 & 0.0000 & 0.0000 & 0.0000 & 0.0000 & 0.0000 \\
\hline SE & 0.0000 & 0.0000 & 0.0000 & 0.0000 & 0.0000 & 0.0000 & 0.0000 \\
\hline ESE & $\odot . \odot \odot \odot \odot$ & $\odot . \odot \odot \odot \odot$ & $\odot . \odot \odot \odot \odot$ & $\odot . \odot \odot \odot \odot$ & $\odot . \odot \odot \odot \odot$ & $\odot . \odot \odot \odot \odot$ & $\odot .0 \odot ० \odot$ \\
\hline$E$ & 0.0000 & 0.0000 & 0.0000 & 0.0000 & 0.0000 & 0.0000 & 0.0000 \\
\hline ENE & $\odot . \odot \odot \odot \odot$ & $\odot .0 \odot \odot \odot$ & $\odot .0 \odot \odot \odot$ & $\odot . \odot \odot \odot \odot$ & $\odot . \odot \odot \odot \odot$ & $\odot .0 \odot \odot \odot$ & $\odot .0000$ \\
\hline $\mathrm{NE}$ & $\odot . \odot \odot \odot \odot$ & $\odot . \odot \odot \odot \odot$ & $\odot . \odot \odot \odot \odot$ & $\odot . \odot \odot \odot \odot$ & $\odot . \odot \odot \odot \odot$ & $\odot . \odot \odot \odot \odot$ & $\odot .0 \odot ० \odot$ \\
\hline NNE & $\odot . \odot \odot \odot \odot$ & $\odot . \odot \odot \odot \odot$ & $\odot . \odot \odot \odot \odot$ & 0.0000 & 0.0000 & 0.0000 & 0.0000 \\
\hline TOTAL & 0.0000 & 0.0000 & 1.0000 & $\odot .0 \odot \odot ०$ & 0.0000 & $0.000 \odot$ & 0.0000 \\
\hline
\end{tabular}

ADDITIONAL WEATHER INFORMATION

Average Air Temperature: $\quad 30.0$ degrees $\mathrm{C}$ $303.16 \mathrm{~K}$

Precipitation: $\quad 1.0 \mathrm{~cm} / \mathrm{y}$ Humidity: $\quad 6.0 \mathrm{~g} / \mathrm{cu} \mathrm{m}$

Lid Height: 3000 meters

Surface Roughness Length: 0.010 meters Height of Wind Measurements: 10.0 meters

Average Wind Speed: $4.373 \mathrm{~m} / \mathrm{s}$

Vertical Temperature Gradients:

$\begin{array}{lll}\text { STABILITY E } & 0.073 \mathrm{k} / \mathrm{m} \\ \text { STABILITY F } & 0.109 \mathrm{k} / \mathrm{m} \\ \text { STABILITY G } & 0.146 \mathrm{k} / \mathrm{m}\end{array}$




\section{ENCLOSURE 5}

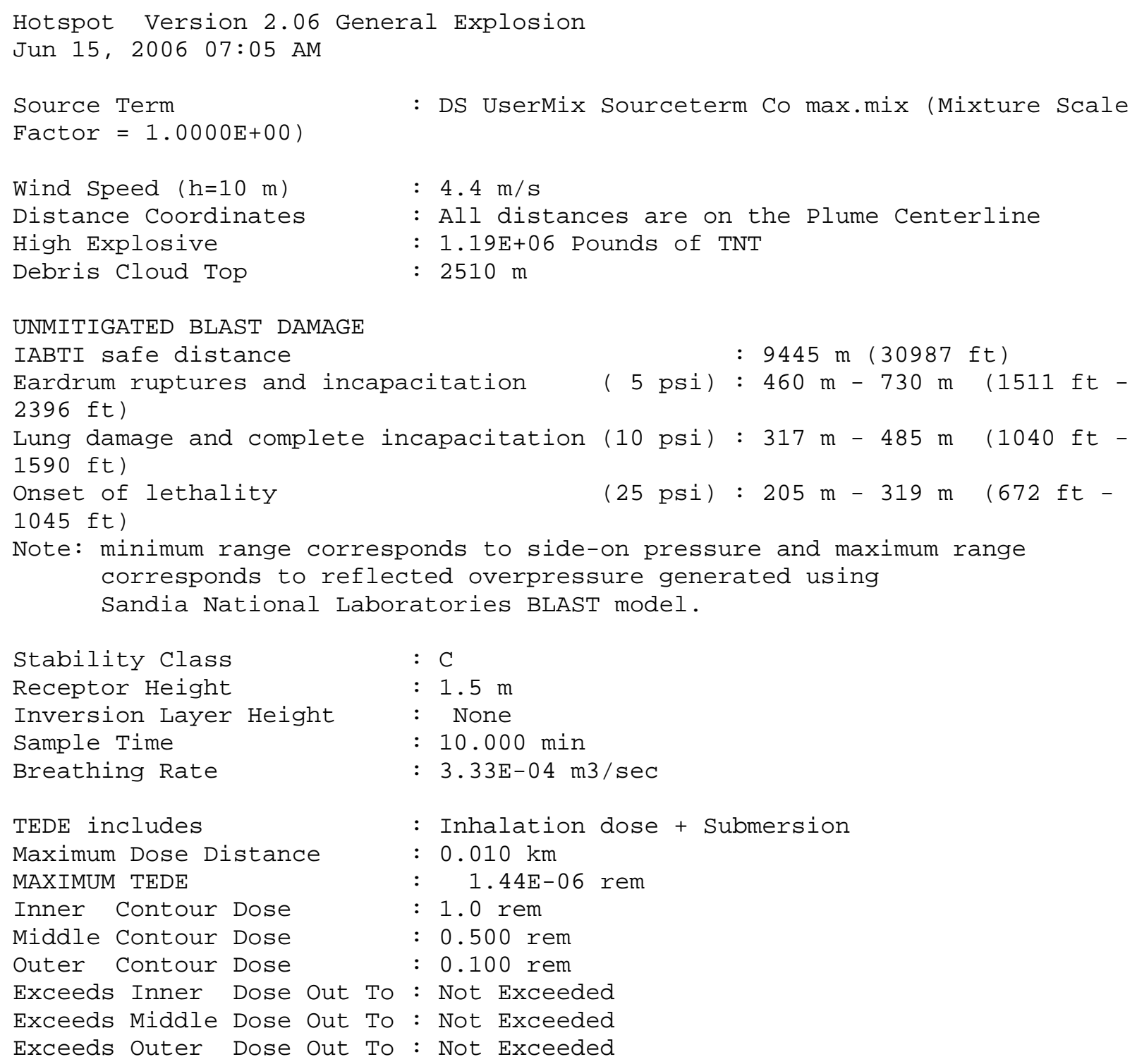




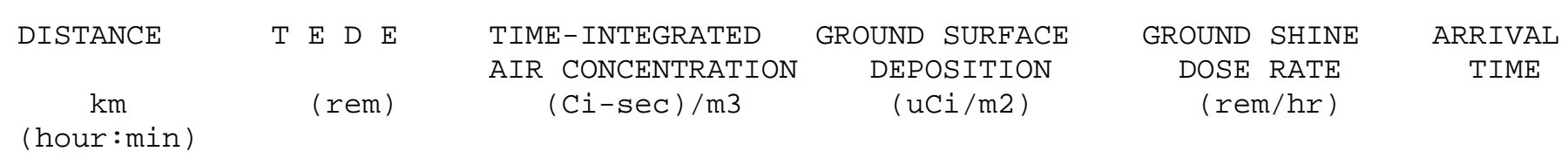

\begin{tabular}{|c|c|c|c|c|c|}
\hline 4.000 & $6.7 \mathrm{E}-\odot 7$ & $1.1 \mathrm{E}-10$ & $3.3 E-07$ & $6.4 \mathrm{E}-13$ & $0 \odot: 17$ \\
\hline $8 . \odot \odot \odot$ & $4.8 E-\odot 7$ & 8. $0 \mathrm{E}-11$ & 2. 3E- -07 & 4. $3 E-13$ & $\odot \odot: 35$ \\
\hline $12 . \odot \odot \odot$ & $3.9 \mathrm{E}-07$ & $6.4 \mathrm{E}-11$ & 1. $9 \mathrm{E}-07$ & $3.4 \mathrm{E}-13$ & $\odot \odot: 53$ \\
\hline $16 . \odot \odot \odot$ & $3.4 \mathrm{E}-07$ & $5.5 \mathrm{E}-11$ & $1.6 \mathrm{E}-07$ & $2.8 \mathrm{E}-13$ & $01: 11$ \\
\hline $2 \odot . \odot \odot \odot$ & $3 . \odot E-\odot 7$ & $4.8 \mathrm{E}-11$ & 1. $4 \mathrm{E}-07$ & $2.4 \mathrm{E}-13$ & $01: 28$ \\
\hline 24.000 & $2.7 \mathrm{E}-\odot 7$ & $4.4 \mathrm{E}-11$ & 1. 3E- - 7 & $2.2 \mathrm{E}-13$ & $01: 46$ \\
\hline 28.000 & $2.5 E-07$ & $4.0 \mathrm{E}-11$ & 1. $2 \mathrm{E}-07$ & 1. $9 \mathrm{E}-13$ & $\odot 2: \odot 4$ \\
\hline $32.00 \odot$ & 2. 3E-๑7 & $3.7 \mathrm{E}-11$ & 1. 1E- -7 & $1.8 \mathrm{E}-13$ & $\odot 2: 22$ \\
\hline $36.0 \odot \odot$ & $2.1 \mathrm{E}-07$ & 3. $4 \mathrm{E}-11$ & $9.9 \mathrm{E}-\odot 8$ & $1.6 \mathrm{E}-13$ & $\odot 2: 4 \odot$ \\
\hline $4 \odot . \odot \odot \odot$ & 2. $\odot E-\odot 7$ & $3.2 \mathrm{E}-11$ & 9. 3E- - & 1. $5 \mathrm{E}-13$ & $\odot 2: 57$ \\
\hline $44.00 \odot$ & 1. $9 \mathrm{E}-\odot 7$ & 3. $0 \mathrm{E}-11$ & $8.7 \mathrm{E}-\odot 8$ & 1. $4 \mathrm{E}-13$ & $\odot 3: 15$ \\
\hline 48.000 & $1.8 \mathrm{E}-07$ & $2.8 \mathrm{E}-11$ & $8.2 \mathrm{E}-08$ & $1.3 \mathrm{E}-13$ & $03: 33$ \\
\hline $52 . \odot \odot \odot$ & 1. 7E- $\odot 7$ & $2.6 \mathrm{E}-11$ & $7.7 \mathrm{E}-\odot 8$ & 1. $2 \mathrm{E}-13$ & $\odot 3: 51$ \\
\hline $56 . \odot \odot \odot$ & $1.6 \mathrm{E}-\odot 7$ & $2.5 \mathrm{E}-11$ & $7.3 E-\odot 8$ & 1. $2 \mathrm{E}-13$ & $\odot 4: \odot 9$ \\
\hline $60.00 \odot$ & $1.5 \mathrm{E}-07$ & $2.4 \mathrm{E}-11$ & $6.9 \mathrm{E}-08$ & 1. $1 \mathrm{E}-13$ & $\odot 4: 26$ \\
\hline $64 . \odot \odot \odot$ & 1. $4 \mathrm{E}-\odot 7$ & 2. $3 E-11$ & $6.6 \mathrm{E}-\odot 8$ & $1.1 \mathrm{E}-13$ & $\odot 4: 44$ \\
\hline $68 . \odot \odot \odot$ & 1. 4E-๑7 & $2.1 \mathrm{E}-11$ & $6.3 E-\odot 8$ & 1. $0 \mathrm{E}-13$ & $\odot 5: \odot 2$ \\
\hline $72.00 \odot$ & 1. $3 \mathrm{E}-07$ & $2.1 \mathrm{E}-11$ & $6.0 \mathrm{E}-\odot 8$ & $9.6 \mathrm{E}-14$ & $05: 20$ \\
\hline $76 . \odot \odot \odot$ & 1. 3E- $\odot 7$ & 2. $. \mathrm{E}-11$ & $5.8 \mathrm{E}-\odot 8$ & $9.2 \mathrm{E}-14$ & $\odot 5: 38$ \\
\hline $8 \odot . \odot \odot \odot$ & 1. $2 \mathrm{E}-\odot 7$ & 1. $9 \mathrm{E}-11$ & $5.5 \mathrm{E}-\odot 8$ & $8.8 \mathrm{E}-14$ & $05: 55$ \\
\hline
\end{tabular}


Hotspot User Mixture Database

User Mixture Name : DS UserMix Sourceterm Co max.mix

Mixture Scale Factor $\quad: 1.0000 \mathrm{E}+00$

Nuclide [01] : Am-241 S 432.2y

Halflife

Inhalation

Submersion

$50-y r$ CEDE

(Years): $4.3220 \mathrm{E}+02$

Ground Shine

Total Activity Released

$(\mathrm{SV} / \mathrm{Bq}): 1.6000 \mathrm{E}-05$

Airborne Fraction

$(\mathrm{Sv}-\mathrm{m} 2) /(\mathrm{Bq}-\mathrm{sec}): 2.3300 \mathrm{E}-17$

Respirable Fraction

(Ci) : $5.7 \mathrm{E}-05$

Respirable Deposition Velocity $(\mathrm{cm} / \mathrm{sec})$

Non-resp. Deposition Velocity $(\mathrm{cm} / \mathrm{sec}): 8.0000 \mathrm{E}+00$

\begin{tabular}{|c|c|c|}
\hline \multicolumn{3}{|c|}{ Nuclide [02] : Co-6๑ S $5.271 \mathrm{y}$} \\
\hline Halflife & (Years) & $5.2710 \mathrm{E}+\odot \odot$ \\
\hline Inhalation & $(\mathrm{Sv} / \mathrm{Bq})$ & $3.0700 \mathrm{E}-\odot 8$ \\
\hline Submersion & $(\mathrm{Sv}-\mathrm{m} 3) /(\mathrm{Bq}-\mathrm{sec})$ & $1.1900 \mathrm{E}-13$ \\
\hline Ground Shine & $(\mathrm{Sv}-\mathrm{m} 2) /(\mathrm{Bq}-\mathrm{sec})$ & $2.3000 \mathrm{E}-15$ \\
\hline Total Activity Released & $(\mathrm{Ci})$ & $7.35 E-5$ \\
\hline Airborne Fraction & & $1.0000 \mathrm{E}+00$ \\
\hline Respirable Fraction & & $1.0000 \mathrm{E}+0 \odot$ \\
\hline Respirable Deposition $V \epsilon$ & elocity (cm/sec) & $3.0000 \mathrm{E}-01$ \\
\hline Non-resp. Deposition V & elocity $(\mathrm{cm} / \mathrm{sec})$ & 8. $\odot \odot \odot \odot E+\odot \odot$ \\
\hline
\end{tabular}

Nuclide [03] : Cs-137 S 30.0y

Halflife

Inhalation

Submersion

$\begin{array}{lll} & (\text { Years }) & : 3.0000 \mathrm{E}+01 \\ & & \end{array}$

Ground Shine

Total Activity Released

$(\mathrm{Sv}-\mathrm{m} 3) /(\mathrm{Bq}-\mathrm{Sec}) \quad: \quad 2.5400 \mathrm{E}-14$

$(\mathrm{Sv}-\mathrm{m} 2) /(\mathrm{Bq}-\mathrm{sec}) \quad: 5.4800 \mathrm{E}-16$

Airborne Fraction

(Ci) : $: 2.4 \mathrm{E}-04$

Respirable Fraction

$: 1.0000 \mathrm{E}+00$

Respirable Deposition Velocity (cm/sec) : 3.0000E-01

Non-resp. Deposition Velocity $(\mathrm{cm} / \mathrm{sec}): 8.0000 \mathrm{E}+00$

Nuclide [04] : Ba-135m S 28.7h

Halflife

(Years) : $3.2763 \mathrm{E}-03$ 


\begin{tabular}{|c|c|c|}
\hline Inhalation & $(\mathrm{Sv} / \mathrm{Bq})$ & $: 3.6000 \mathrm{E}-10$ \\
\hline Submersion & $(\mathrm{Sv}-\mathrm{m} 3) /(\mathrm{Bq}-\mathrm{sec})$ & $2.1600 \mathrm{E}-15$ \\
\hline Ground Shine & $(\mathrm{Sv}-\mathrm{m} 2) /(\mathrm{Bq}-\mathrm{sec})$ & $5.3800 \mathrm{E}-17$ \\
\hline Total Activity Released & $(\mathrm{Ci})$ & $2.4 \mathrm{E}-04$ \\
\hline Airborne Fraction & & $: 1.0000 \mathrm{E}+00$ \\
\hline Respirable Fraction & & 1. $\odot \odot \odot \odot E+\odot \odot$ \\
\hline Respirable Deposition & $(\mathrm{cm} / \mathrm{sec})$ & $3.0 \odot \odot \odot E-\odot$ \\
\hline Non-resp. Deposition V & $(\mathrm{cm} / \mathrm{sec})$ & $8 . \odot \odot \odot \odot E+\odot$ \\
\hline
\end{tabular}

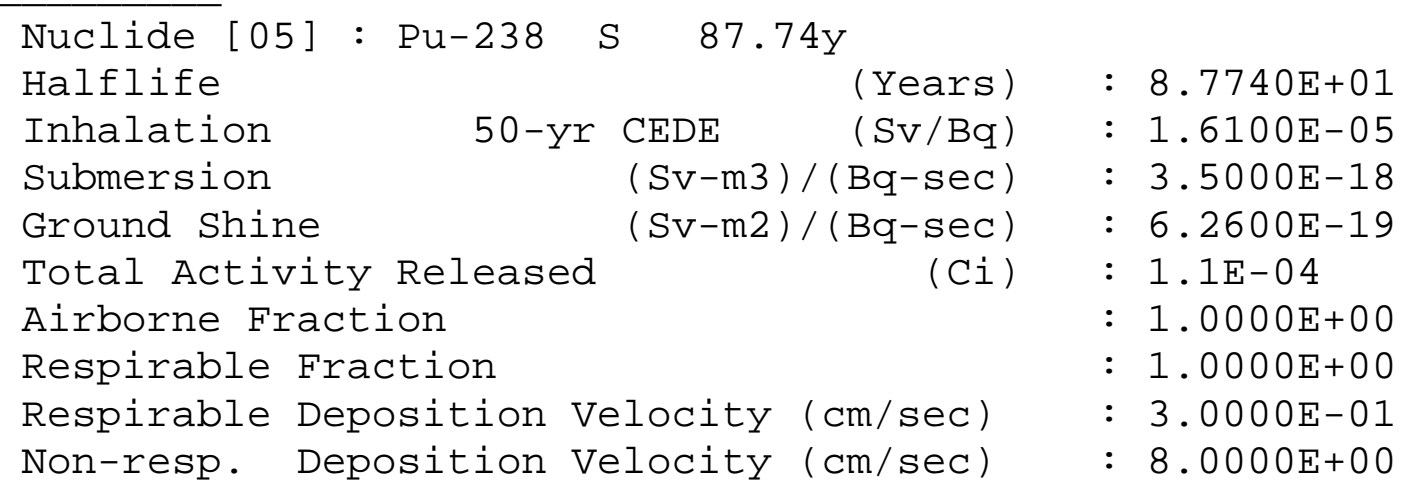

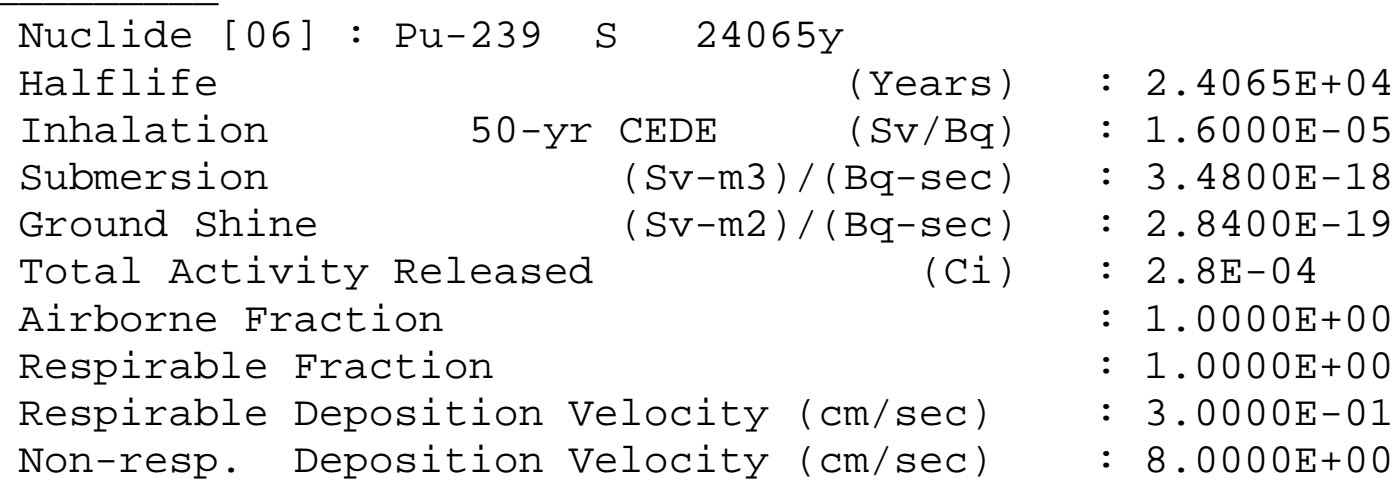

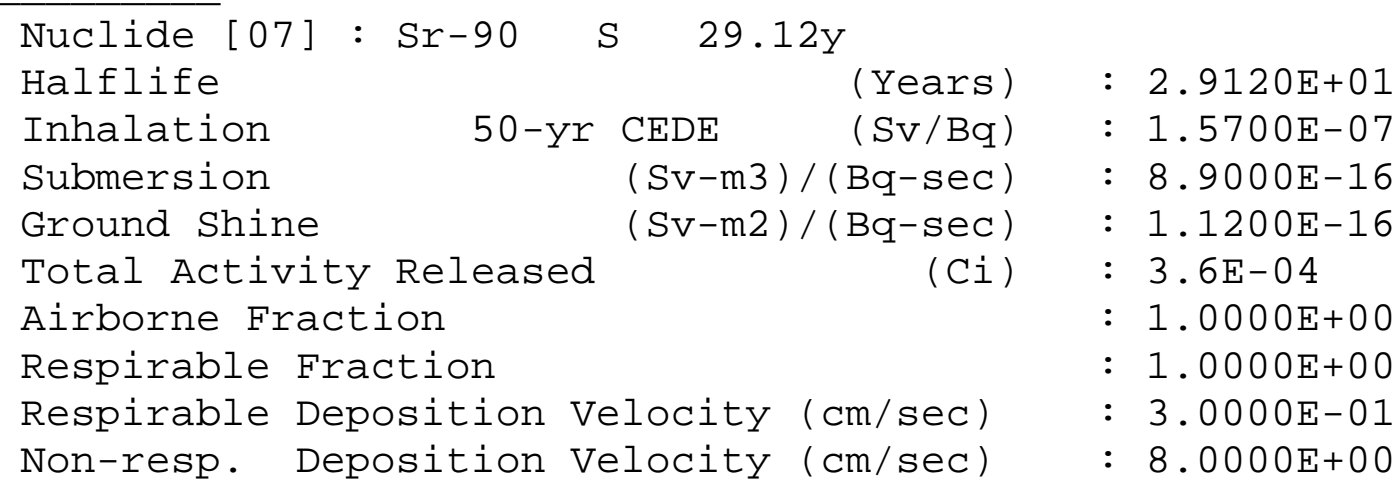




\begin{tabular}{|c|c|c|}
\hline \multicolumn{3}{|c|}{ Nuclide [๑8] : Y-9๑ S $\quad 64 . \odot \mathrm{h}$} \\
\hline Halflife & (Years) & $7.3059 \mathrm{E}-\odot 3$ \\
\hline Inhalation & (Sv/Bq) & $1.500 \odot E-\odot 9$ \\
\hline Submersion & $(\mathrm{Sv}-\mathrm{m} 3) /(\mathrm{Bq}-\mathrm{sec})$ & $7.9200 \mathrm{E}-16$ \\
\hline Ground Shine & $(\mathrm{Sv}-\mathrm{m} 2) /(\mathrm{Bq}-\mathrm{sec})$ & $1.1000 \mathrm{E}-16$ \\
\hline Total Activity Released & $(\mathrm{Ci})$ & $3.6 \mathrm{E}-04$ \\
\hline Airborne Fraction & & 1. $\odot \odot \odot \odot E+\odot \odot$ \\
\hline Respirable Fraction & & 1. $\odot \odot \odot \odot E+\odot \odot$ \\
\hline Respirable Deposition V & elocity $(\mathrm{cm} / \mathrm{sec})$ & 3. $0 \odot \odot \odot E-\odot 1$ \\
\hline Non-resp. Deposition Vt & elocity $(\mathrm{cm} / \mathrm{sec})$ & : $8.00 \odot \odot E+\odot \odot$ \\
\hline
\end{tabular}

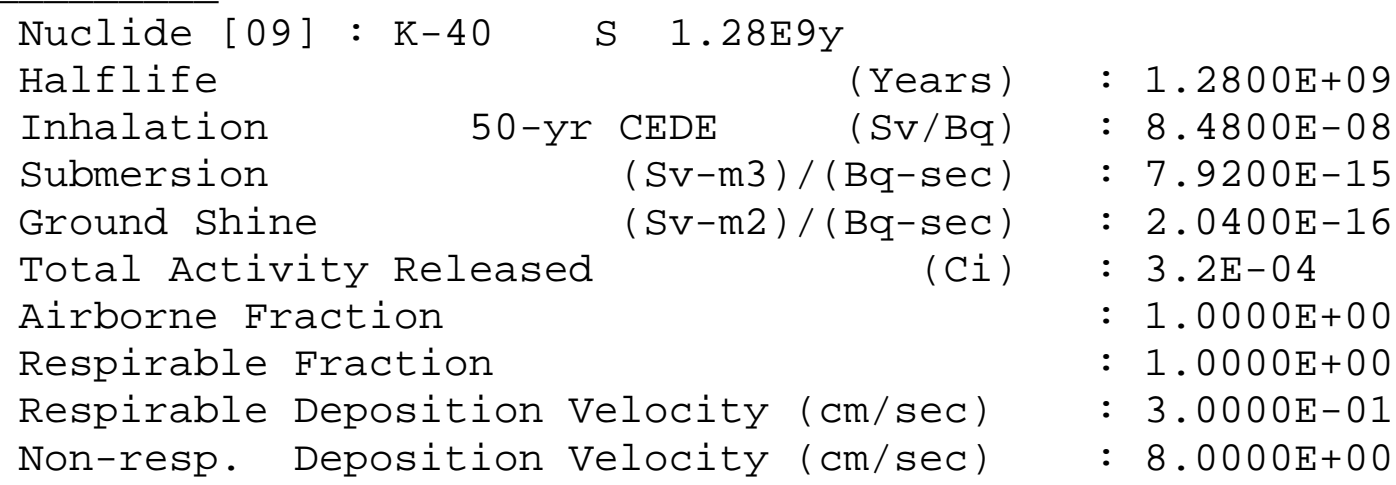

\begin{tabular}{|c|c|c|}
\hline Nuclide [10] : Th-232 & S1. 405E10y & \\
\hline Halflife & (Years) & $1.4050 \mathrm{E}+10$ \\
\hline Inhalation & CEDE $\quad(\mathrm{S} v / \mathrm{Bq})$ & $2.4800 \mathrm{E}-\odot 5$ \\
\hline Submersion & $(\mathrm{Sv}-\mathrm{m} 3) /(\mathrm{Bq}-\mathrm{sec})$ & $7.2400 \mathrm{E}-18$ \\
\hline Ground Shine & $(\mathrm{Sv}-\mathrm{m} 2) /(\mathrm{Bq}-\mathrm{sec})$ & $4.5500 \mathrm{E}-19$ \\
\hline Total Activity Released & $(\mathrm{Ci})$ & $5.400 E-\odot 5$ \\
\hline Airborne Fraction & & $: 1.0000 \mathrm{E}+00$ \\
\hline Respirable Fraction & & 1. $\odot \odot \odot \odot E+\odot \odot$ \\
\hline Respirable Deposition & elocity (cm/sec) & 3. $.0 \odot \odot E-\odot 1$ \\
\hline Non-resp. Deposition & elocity ( $\mathrm{cm} / \mathrm{sec})$ & $8 . \odot \odot \odot \odot E+\odot \odot$ \\
\hline
\end{tabular}

\begin{tabular}{|c|c|c|c|}
\hline Nuclide [11] & $\mathrm{Ra}-228$ & $5.75 y$ & \\
\hline Halflife & & (Years) & $: 5.7500 E+\odot \odot$ \\
\hline Inhalation & $50-y r$ & $(\mathrm{~Sv} / \mathrm{Bq})$ & $: 1.6000 \mathrm{E}-05$ \\
\hline Submersion & & $(\mathrm{Sv}-\mathrm{m} 3) /(\mathrm{Bq}-\mathrm{sec})$ & $\odot . \odot \odot \odot \odot E+\odot \odot$ \\
\hline Ground Shine & & $(\mathrm{Sv}-\mathrm{m} 2) /(\mathrm{Bq}-\mathrm{sec})$ & $: \quad \odot .0 \odot \odot \odot E+\odot \odot$ \\
\hline Total Activit & Released & $(\mathrm{Ci})$ & : $5.400 \odot E-\odot 5$ \\
\hline
\end{tabular}


Airborne Fraction

Respirable Fraction

Respirable Deposition Velocity $(\mathrm{cm} / \mathrm{sec})$

Non-resp. Deposition Velocity $(\mathrm{cm} / \mathrm{sec})$
: $1.0000 \mathrm{E}+00$

$: 1.0000 \mathrm{E}+00$

: $3.0000 \mathrm{E}-01$

: $8.0000 \mathrm{E}+\odot \odot$

\begin{tabular}{|c|c|c|}
\hline Nuclide [12] & $6.13 \mathrm{~h}$ & \\
\hline Halflife & (Years) & $6.9977 \mathrm{E}-04$ \\
\hline Inhalation & CEDE $\quad(\mathrm{S} v / \mathrm{Bq})$ & 1. $4600 \mathrm{E}-\odot 8$ \\
\hline Submersion & $(\mathrm{Sv}-\mathrm{m} 3) /(\mathrm{Bq}-\mathrm{sec})$ & 4. $4900 \mathrm{E}-14$ \\
\hline Ground Shine & $(\mathrm{Sv}-\mathrm{m} 2) /(\mathrm{Bq}-\mathrm{sec})$ & $9.3900 \mathrm{E}-16$ \\
\hline Total Activity Released & $(\mathrm{Ci})$ & $5.4000 \mathrm{E}-05$ \\
\hline Airborne Fraction & & 1. $\odot \odot \odot \odot E+\odot \odot$ \\
\hline Respirable Fraction & & 1. $\odot \odot \odot \odot E+\odot \odot$ \\
\hline Respirable Deposition Ve & elocity $(\mathrm{cm} / \mathrm{sec})$ & $3 . \odot \odot \odot \odot E-\odot 1$ \\
\hline Non-resp. Deposition Ve & $(\mathrm{cm} / \mathrm{sec})$ & 8. $\odot \odot \odot \odot E+\odot \odot$ \\
\hline
\end{tabular}

Nuclide [13] : Th-228 S 1.9131y

Halflife

Inhalation

Submersion

$50-y r$ CEDE $\quad$ (Years) $: 1.9131 \mathrm{E}+00$

Ground Shine

Total Activity Released

$(\mathrm{Sv}-\mathrm{m} 3) /(\mathrm{Bq}-\mathrm{sec}) \quad: \quad 8.1000 \mathrm{E}-17$

Airborne Fraction

$(\mathrm{Sv}-\mathrm{m} 2) /(\mathrm{Bq}-\mathrm{Sec}) \quad: \quad 2.1300 \mathrm{E}-18$

Respirable Fraction

(Ci) : $5.4000 \mathrm{E}-05$

Respirable Deposition

Non-resp. Deposition Velocity $(\mathrm{cm} / \mathrm{sec}): 8.0000 \mathrm{E}+0 \odot$

: $1.0000 \mathrm{E}+00$

$: 1.0000 \mathrm{E}+00$

: 3. $0000 \mathrm{E}-01$

\begin{tabular}{|c|c|c|}
\hline Nuclide [14] & $3.66 \mathrm{~d}$ & \\
\hline Halflife & (Years) & 1. $\odot \odot 27 E-\odot 2$ \\
\hline Inhalation & $(\mathrm{S} v / \mathrm{Bq})$ & $3.3600 \mathrm{E}-\odot 6$ \\
\hline Submersion & $(\mathrm{Sv}-\mathrm{m} 3) /(\mathrm{Bq}-\mathrm{sec})$ & $4.2900 \mathrm{E}-16$ \\
\hline Ground Shine & $(\mathrm{Sv}-\mathrm{m} 2) /(\mathrm{Bq}-\mathrm{sec})$ & $9.1500 \mathrm{E}-18$ \\
\hline Total Activity Released & $(\mathrm{Ci})$ & $: 5.4000 \mathrm{E}-05$ \\
\hline Airborne Fraction & & $: 1.000 \odot E+\odot \odot$ \\
\hline Respirable Fraction & & 1. $\odot \odot \odot \odot E+\odot \odot$ \\
\hline Respirable Deposition & elocity $(\mathrm{cm} / \mathrm{sec})$ & $: \quad 3.0000 \mathrm{E}-01$ \\
\hline Non-resp. Deposition & $(\mathrm{cm} / \mathrm{sec})$ & $: 8.0000 \mathrm{E}+0 \odot$ \\
\hline
\end{tabular}

Nuclide [15] : Po-216 Halflife
$0.15 \mathrm{~s}$

(Years) : 4.7532E-๑9 


\begin{tabular}{|c|c|c|}
\hline Inhalation & $(\mathrm{Sv} / \mathrm{Bq})$ & $: 0.0000 \mathrm{E}+00$ \\
\hline Submersion & $(\mathrm{Sv}-\mathrm{m} 3) /(\mathrm{Bq}-\mathrm{sec})$ & $7.7500 E-19$ \\
\hline Ground Shine & $(\mathrm{Sv}-\mathrm{m} 2) /(\mathrm{Bq}-\mathrm{sec})$ & 1. $6100 \mathrm{E}-20$ \\
\hline Total Activity Released & (Ci) & $5.4000 E-05$ \\
\hline Airborne Fraction & & 1. $\odot \odot \odot \odot E+\odot \odot$ \\
\hline Respirable Fraction & & 1. $\odot \odot \odot \odot E+\odot \odot$ \\
\hline Respirable Deposition & $(\mathrm{cm} / \mathrm{sec})$ & 3. $\odot \odot \odot \odot E-\odot 1$ \\
\hline Deposition V & Velocity & 8. $\odot \odot \odot \odot E+\odot \odot$ \\
\hline
\end{tabular}

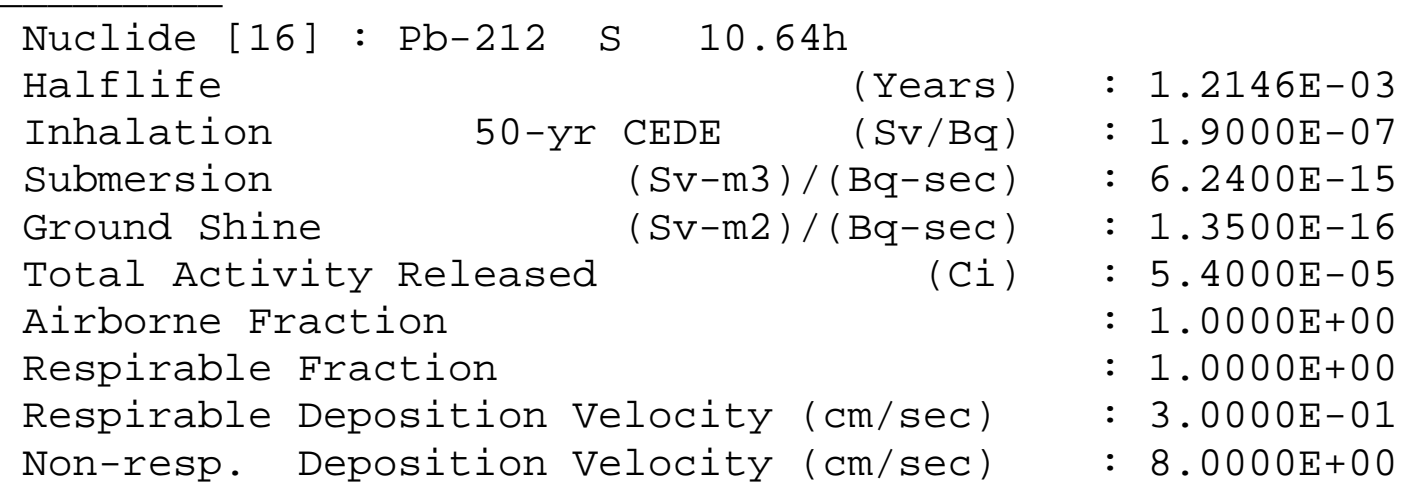

\begin{tabular}{|c|c|c|}
\hline Nuclide [17] : Bi-212 & $60.55 \mathrm{~m}$ & \\
\hline Halflife & (Years) & 1. $1520 \mathrm{E}-04$ \\
\hline Inhalation & $(\mathrm{Sv} / \mathrm{Bq})$ & $3.3200 E-08$ \\
\hline Submersion & $(\mathrm{Sv}-\mathrm{m} 3) /(\mathrm{Bq}-\mathrm{sec})$ & $8.9500 \mathrm{E}-15$ \\
\hline Ground Shine & $(\mathrm{Sv}-\mathrm{m} 2) /(\mathrm{Bq}-\mathrm{sec})$ & $2.2500 \mathrm{E}-16$ \\
\hline Total Activity Released & $(\mathrm{Ci})$ & \\
\hline Airborne Fraction & & $\partial \odot \mathrm{E}+\odot \odot$ \\
\hline Respirable Fraction & & $\partial \mathrm{E}+00$ \\
\hline Respirable Deposit & elocity (cm/sec) & $3.0000 \mathrm{E}-01$ \\
\hline Non-resp. Deposition & elocity ( $\mathrm{cm} / \mathrm{sec})$ & $: \quad 8.0000 \mathrm{E}+0 \odot$ \\
\hline
\end{tabular}

\begin{tabular}{|c|c|c|}
\hline Nuclide [18] : Po-212 & $0.305 u$ & \\
\hline Halflife & (Years) & $3.0500 E-01$ \\
\hline Inhalation & $(\mathrm{Sv} / \mathrm{Bq})$ & $\odot .0 \odot \odot \odot E+\odot \odot$ \\
\hline Submersion & $(\mathrm{Sv}-\mathrm{m} 3) /(\mathrm{Bq}-\mathrm{sec})$ & $\odot . \odot \odot \odot \odot E+\odot \odot$ \\
\hline Ground Shine & $(\mathrm{Sv}-\mathrm{m} 2) /(\mathrm{Bq}-\mathrm{sec})$ & $\odot .0000 E+00$ \\
\hline Total Activity Released & $(\mathrm{Ci})$ & $: 3$ \\
\hline Airborne Fraction & & : $1.0000 \mathrm{E}+0 \odot$ \\
\hline Respirable Fraction & & : $1.00 \odot \odot E+\odot \odot$ \\
\hline Respirable Deposition Ve & locity ( $\mathrm{cm} / \mathrm{sec})$ & $: \quad 3.0000 \mathrm{E}-01$ \\
\hline Non-resp. Deposition Ve & locity $(\mathrm{cm} / \mathrm{sec})$ & : $8.0000 \mathrm{E}+\odot \odot$ \\
\hline
\end{tabular}




\begin{tabular}{|c|c|c|}
\hline Nuclide [19] & $3.07 \mathrm{~m}$ & \\
\hline Halflife & (Years) & $5.8409 E-06$ \\
\hline Inhalation & $(\mathrm{Sv} / \mathrm{Bq})$ & $\odot . \odot \odot \odot \odot E+\odot \odot$ \\
\hline Submersion & $(\mathrm{Sv}-\mathrm{m} 3) /(\mathrm{Bq}-\mathrm{sec})$ & 1. $6900 \mathrm{E}-13$ \\
\hline Ground Shine & $(\mathrm{Sv}-\mathrm{m} 2) /(\mathrm{Bq}-\mathrm{sec})$ & $2.9700 \mathrm{E}-15$ \\
\hline Total Activity Released & $(\mathrm{Ci})$ & 1. $9000 \mathrm{E}-05$ \\
\hline Airborne Fraction & & 1. $.000 \mathrm{E}+\odot \odot$ \\
\hline Respirable Fraction & & 1. $.0 \odot \odot E+\odot \odot$ \\
\hline able Deposition & elocity $(\mathrm{cm} / \mathrm{s}$ & 3. $0000 \mathrm{E}-01$ \\
\hline - Deposition V & elocity ( $\mathrm{cm} / \mathrm{sec})$ & $: \quad 8.0000 \mathrm{E}+\odot \odot$ \\
\hline
\end{tabular}

\begin{tabular}{|c|c|c|}
\hline $\begin{array}{l}\text { Nuclide [20] : U-235 } \\
\text { Halflife }\end{array}$ & $\begin{array}{l}\text { S 703.8E6y } \\
\text { (Years) }\end{array}$ & $7.0380 \mathrm{E}+08$ \\
\hline Inhalation & CEDE $\quad(\mathrm{Sv} / \mathrm{Bq})$ & $8.4700 \mathrm{E}-06$ \\
\hline Submersion & $(\mathrm{Sv}-\mathrm{m} 3) /(\mathrm{Bq}-\mathrm{sec})$ & $6.4600 \mathrm{E}-15$ \\
\hline Ground Shine & $(\mathrm{Sv}-\mathrm{m} 2) /(\mathrm{Bq}-\mathrm{sec})$ & 1. $4000 \mathrm{E}-16$ \\
\hline Total Activity Released & $(\mathrm{Ci})$ & $: \quad 4.8000 \mathrm{E}-07$ \\
\hline Airborne Fraction & & $: 1.0000 \mathrm{E}+00$ \\
\hline Respirable Fraction & & $1.0000 E+\odot \odot$ \\
\hline irable Deposition & elocity & 3. $0000 \mathrm{E}-01$ \\
\hline Non-resp. Deposition V & elocity ( $\mathrm{cm} / \mathrm{sec})$ & : $8.0000 \mathrm{E}+\odot \odot$ \\
\hline
\end{tabular}

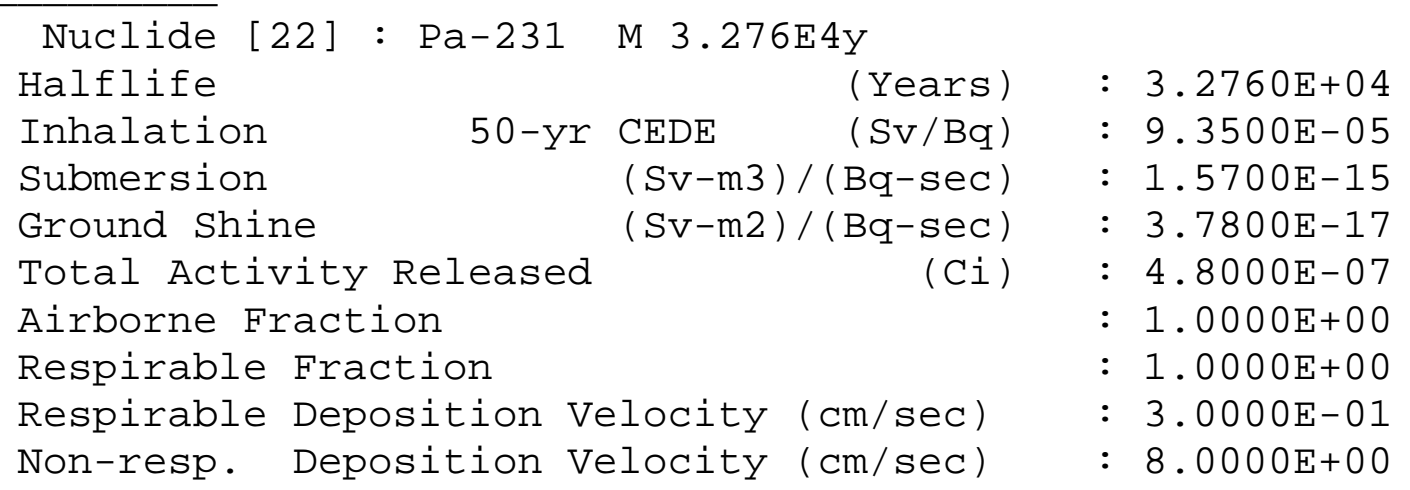

Nuclide [23] : Ac-227 S 21.773y

Halflife (Years) : 2.1773E +01

Inhalation 50-yr CEDE (Sv/Bq) : 5.5300E-05

Submersion $\quad(\mathrm{Sv}-\mathrm{m3}) /(\mathrm{Bq}-\mathrm{sec}): 5.1200 \mathrm{E}-18$

Ground Shine $\quad(\mathrm{Sv}-\mathrm{m} 2) /(\mathrm{Bq}-\mathrm{sec}): 1.4100 \mathrm{E}-19$

Total Activity Released (Ci) : 4.8000E-07 
Airborne Fraction

Respirable Fraction

Respirable Deposition Velocity ( $\mathrm{cm} / \mathrm{sec}$ )

Non-resp. Deposition Velocity (cm/sec)
: $1.0000 \mathrm{E}+\odot \odot$

: $1.0000 \mathrm{E}+00$

: $3.0000 \mathrm{E}-01$

: $8.0 \odot \odot \odot E+\odot \odot$

Nuclide [24] : Th-227 S 18.718d

Halflife

Inhalation

Submersion

Ground Shine

Total Activity Released

Airborne Fraction

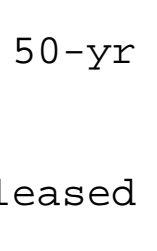

Respirable Fraction

Respirable Deposition Velocity (cm/sec)

Non-resp. Deposition Velocity (cm/sec)
(Years ) : $5.1282 \mathrm{E}-02$

$(\mathrm{Sv} / \mathrm{Bq}): 1.0400 \mathrm{E}-05$

$(\mathrm{Sv}-\mathrm{m} 3) /(\mathrm{Bq}-\mathrm{sec}) \quad: 4.4300 \mathrm{E}-15$

$(\mathrm{Sv}-\mathrm{m} 2) /(\mathrm{Bq}-\mathrm{sec}) \quad: 9.8100 \mathrm{E}-17$

(Ci) : $4.8000 \mathrm{E}-07$

: $1.0 \odot \odot \odot E+\odot \odot$

: $1.0000 \mathrm{E}+00$

: $3.0 \odot \odot \odot E-01$

: $8.0000 \mathrm{E}+\odot \odot$

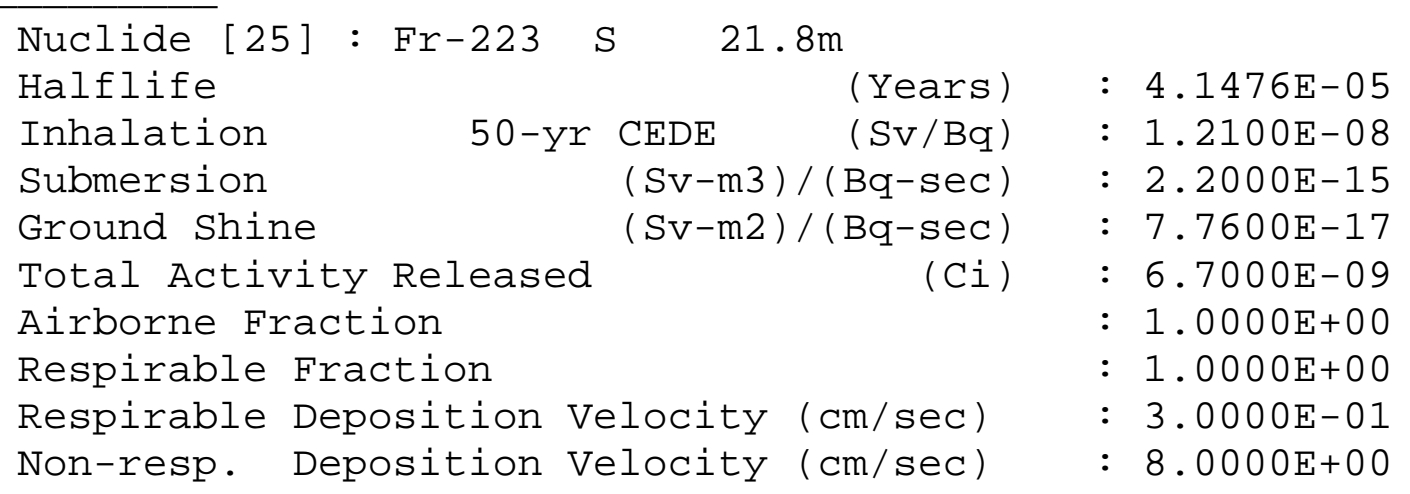

Nuclide [26] : Ra-223 S 11.434d

Halflife $\quad$ (Years) : 3.1326E-02

Inhalation 50-yr CEDE (Sv/Bq) : 8.6800E-06

Submersion $\quad($ Sv-m3 $) /(B q-S e c): 5.4700 E-15$

Ground Shine $\quad(S v-m 2) /(B q-s e c): 1.2100 E-16$

Total Activity Released (Ci) : 4.8000E-07

Airborne Fraction $\quad: 1.0000 \mathrm{E}+0 \odot$

Respirable Fraction : $1.0000 \mathrm{E}+00$

Respirable Deposition Velocity (cm/sec) : $3.0000 \mathrm{c}-01$

Non-resp. Deposition Velocity (cm/sec) : 8.0000E+००

Nuclide $[27]:$ Rn-219 $3.96 s$
Halflife




\begin{tabular}{|c|c|c|}
\hline Inhalation & $(\mathrm{Sv} / \mathrm{Bq})$ & $: 0.0000 E+00$ \\
\hline Submersion & $(\mathrm{Sv}-\mathrm{m} 3) /(\mathrm{Bq}-\mathrm{sec})$ & $2.4600 \mathrm{E}-15$ \\
\hline Ground Shine & $(\mathrm{Sv}-\mathrm{m} 2) /(\mathrm{Bq}-\mathrm{sec})$ & $5.2800 \mathrm{E}-17$ \\
\hline Total Activity Released & $(\mathrm{Ci})$ & $4.8000 \mathrm{E}$ \\
\hline Airborne Fraction & & 1. $0000 \mathrm{E}+00$ \\
\hline Respirable Fraction & & 1. $\odot \odot \odot \odot E+\odot \odot$ \\
\hline Respirable Deposition & $(\mathrm{cm} / \mathrm{sec})$ & $\odot . \odot \odot \odot \odot E+\odot \odot$ \\
\hline Non-resp. Deposition V & $(\mathrm{cm} / \mathrm{sec})$ & $\odot . \odot \odot \odot \odot E+\odot \odot$ \\
\hline
\end{tabular}

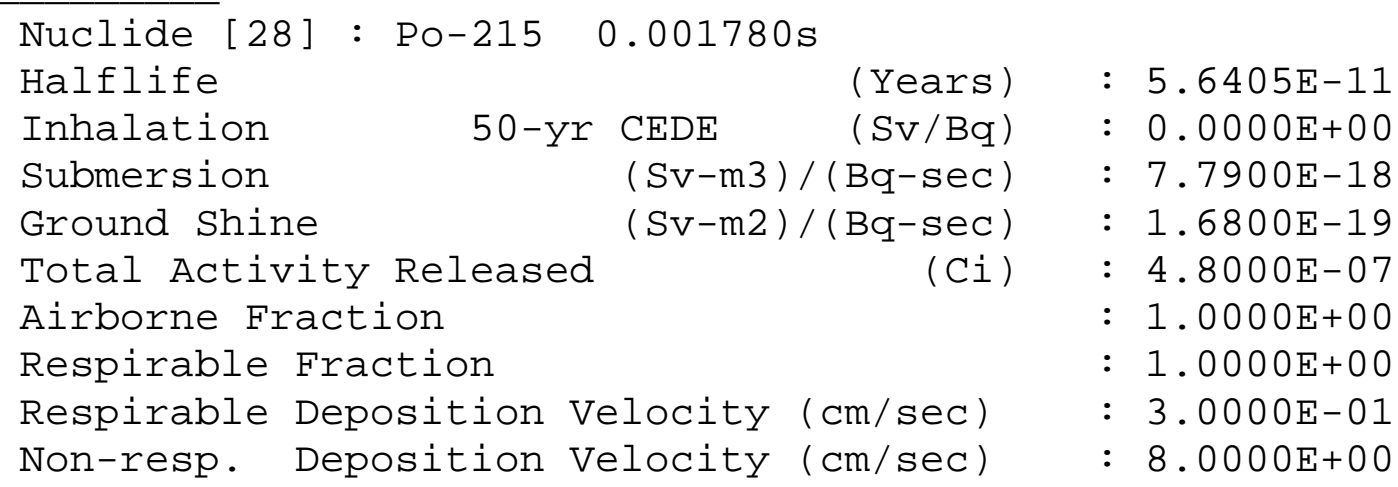

\begin{tabular}{|c|c|c|}
\hline $\begin{array}{l}\text { Nuclide [29] : Pb-211 } \\
\text { Halflife }\end{array}$ & $\begin{array}{l}36.1 \mathrm{~m} \\
\text { (Years) }\end{array}$ & $6.8683 E-\odot 5$ \\
\hline Inhalation & $(\mathrm{Sv} / \mathrm{Bq})$ & 1. 2000E - $\odot 8$ \\
\hline Submersion & $(\mathrm{Sv}-\mathrm{m} 3) /(\mathrm{Bq}-\mathrm{sec})$ & $2.5900 \mathrm{E}-15$ \\
\hline Ground Shine & $(\mathrm{Sv}-\mathrm{m} 2) /(\mathrm{Bq}-\mathrm{sec})$ & $9.5000 \mathrm{E}-17$ \\
\hline Total Activity Released & $(\mathrm{Ci})$ & 4.80 \\
\hline Airborne Fraction & & : $1.0000 \mathrm{E}+00$ \\
\hline Respirable Fraction & & 1. $.000 \mathrm{E}+0 \odot$ \\
\hline le Deposition & & $3.0000 \mathrm{E}-\odot$ \\
\hline . Deposition Ve & elocity ( $\mathrm{cm} / \mathrm{sec})$ & $: \quad 8.0000 \mathrm{E}+\odot$ \\
\hline
\end{tabular}

\begin{tabular}{|c|c|c|}
\hline Nuclide [30] & $2.14 \mathrm{~m}$ & \\
\hline Halflife & (Years) & $: 4.0715 E-06$ \\
\hline Inhalation & CEDE $\quad(\mathrm{Sv} / \mathrm{Bq})$ & $\odot . \odot \odot \odot \odot E+\odot \odot$ \\
\hline Submersion & $(\mathrm{Sv}-\mathrm{m} 3) /(\mathrm{Bq}-\mathrm{sec})$ & $2.040 \odot E-15$ \\
\hline Ground Shine & $(\mathrm{Sv}-\mathrm{m} 2) /(\mathrm{Bq}-\mathrm{sec})$ & $: 4.4000 \mathrm{E}-17$ \\
\hline Total Activity Released & $(\mathrm{Ci})$ & $: \quad 4.8000 \mathrm{E}-07$ \\
\hline Airborne Fraction & & 1. $0000 \mathrm{E}+00$ \\
\hline Respirable Fraction & & : $1.0000 \mathrm{E}+\odot \odot$ \\
\hline able Deposition & & $3.0000 E-01$ \\
\hline p. Deposition $\mathrm{V}$ & $(\mathrm{cm} / \mathrm{sec})$ & $8 . \odot \odot ० \odot E+\odot$ \\
\hline
\end{tabular}




\begin{tabular}{|c|c|c|}
\hline Nuclide [31] : Tl-207 & $4.77 \mathrm{~m}$ & \\
\hline Halflife & (Years) & $: 9.0753 E-06$ \\
\hline Inhalation & $(\mathrm{Sv} / \mathrm{Bq})$ & $\odot .00 \odot \odot E+\odot \odot$ \\
\hline Submersion & $(\mathrm{Sv}-\mathrm{m} 3) /(\mathrm{Bq}-\mathrm{sec})$ & $4.5300 \mathrm{E}-16$ \\
\hline Ground Shine & $(\mathrm{Sv}-\mathrm{m} 2) /(\mathrm{Bq}-\mathrm{sec})$ & $5.5600 \mathrm{E}-17$ \\
\hline Total Activity Released & $(\mathrm{Ci})$ & 4. 80००E- - 7 \\
\hline Airborne Fraction & & $1.000 \odot E+\odot \odot$ \\
\hline Respirable Fraction & & $1.0000 \mathrm{E}+\odot \odot$ \\
\hline Respirable Deposition & elocity $(\mathrm{cm} / \mathrm{sec})$ & 3. $00 \odot \odot E-\odot 1$ \\
\hline Non-resp. Deposition & elocity $(\mathrm{cm} / \mathrm{sec})$ & $: \quad 8.000 \odot E+\odot \odot$ \\
\hline
\end{tabular}

\begin{tabular}{|c|c|c|}
\hline $\begin{array}{l}\text { Juclide [32] : } \\
\text { talflife }\end{array}$ & $0.516 \mathrm{~s}$ & \\
\hline $\begin{array}{l}\text { lalflife } \\
\text { nhalation }\end{array}$ & $\begin{array}{l}\text { (Years) } \\
(\mathrm{Sv} / \mathrm{Bq})\end{array}$ & $: 1.6351 \mathrm{E}-\mathrm{e}$ \\
\hline Submersion & $(\mathrm{Sv}-\mathrm{m} 3) /(\mathrm{Bq}-\mathrm{sec})$ & $: 3.5600 \mathrm{E}-16$ \\
\hline Ground Shine & $(\mathrm{Sv}-\mathrm{m} 2) /(\mathrm{Bq}-\mathrm{sec})$ & 7.4 \\
\hline Total Activity Released & $(\mathrm{Ci})$ & $1.3000 \mathrm{E}$ \\
\hline Airborne Fraction & & $00 \mathrm{E}+0 \odot$ \\
\hline Respirable Fraction & & $1.0000 \mathrm{E}+\odot \odot$ \\
\hline ble Deposition & & \\
\hline risia & & 8. $\odot \odot \odot \odot Е+$ \\
\hline
\end{tabular}

\begin{tabular}{|c|c|c|}
\hline Halflife & (Years) & $4.4680 E+09$ \\
\hline Inhalation & CEDE $\quad(\mathrm{S} v / \mathrm{Bq})$ & $8.0400 E-06$ \\
\hline Submersion & $(\mathrm{Sv}-\mathrm{m} 3) /(\mathrm{Bq}-\mathrm{sec})$ & $2.5000 E-18$ \\
\hline Ground Shine & $(\mathrm{Sv}-\mathrm{m} 2) /(\mathrm{Bq}-\mathrm{sec})$ & $4.2300 \mathrm{E}-19$ \\
\hline Total Activity Released & $(\mathrm{Ci})$ & $6.10 \odot \odot E-\odot 5$ \\
\hline Airborne Fraction & & $1.0 \odot \odot \odot E+\odot \odot$ \\
\hline Respirable Fraction & & 1. $\odot \odot \odot \odot E+\odot \odot$ \\
\hline Respirable Deposition & locity $(\mathrm{cm} / \mathrm{sec})$ & 3. $0000 \mathrm{E}-01$ \\
\hline Non-resp. Deposition & $(\mathrm{cm} / \mathrm{sec})$ & 8. $\odot \odot \odot \odot E+\odot \odot$ \\
\hline
\end{tabular}

\begin{tabular}{|c|c|c|c|}
\hline \multicolumn{4}{|l|}{ Nuclide [34] } \\
\hline Halflife & & (Years) & $: \quad 6.6027 \mathrm{E}-02$ \\
\hline Inhalation & $50-y r$ & CEDE $\quad(\mathrm{S} v / \mathrm{Bq})$ & : $7.6900 \mathrm{E}-\odot 9$ \\
\hline Submersion & & $(\mathrm{Sv}-\mathrm{m} 3) /(\mathrm{Bq}-\mathrm{sec})$ & $: 2.9400 \mathrm{E}-16$ \\
\hline Ground Shine & & $(\mathrm{Sv}-\mathrm{m} 2) /(\mathrm{Bq}-\mathrm{sec})$ & $: 7.4900 \mathrm{E}-18$ \\
\hline Total Activity & Released & $(\mathrm{Ci})$ & : $6.1000 \mathrm{E}-\odot 5$ \\
\hline
\end{tabular}


Airborne Fraction

Respirable Fraction

Respirable Deposition Velocity $(\mathrm{cm} / \mathrm{sec})$

Non-resp. Deposition Velocity $(\mathrm{cm} / \mathrm{sec})$
: $1.0000 \mathrm{E}+00$

: $1.0000 \mathrm{E}+00$

: $3.0000 \mathrm{E}-01$

: $8.0000 \mathrm{E}+\odot \odot$

\begin{tabular}{|c|c|c|}
\hline Nuclide [35] : Pa-234m & $1.17 \mathrm{~m}$ & \\
\hline Halflife & (Years) & $2.2260 \mathrm{E}-\odot 6$ \\
\hline Inhalation & $(\mathrm{Sv} / \mathrm{Bq})$ & $\odot .0 \odot \odot \odot E+\odot \odot$ \\
\hline Submersion & $(\mathrm{Sv}-\mathrm{m} 3) /(\mathrm{Bq}-\mathrm{sec})$ & 1. $2100 \mathrm{E}-15$ \\
\hline Ground Shine & $(\mathrm{Sv}-\mathrm{m} 2) /(\mathrm{Bq}-\mathrm{sec})$ & 1. $0800 \mathrm{E}-16$ \\
\hline Total Activity Released & $(\mathrm{Ci})$ & $: 6.1000 \mathrm{E}-05$ \\
\hline Airborne Fraction & & $: 1.0000 \mathrm{E}+\odot \odot$ \\
\hline Respirable Fraction & & 1. $\odot \odot \odot \odot E+\odot \odot$ \\
\hline Respirable Deposition & elocity $(\mathrm{cm} / \mathrm{sec})$ & 3. $0 \odot \odot \odot E-\odot 1$ \\
\hline Non-resp. Deposition & $(\mathrm{cm} / \mathrm{sec})$ & 8. $\odot \odot \odot \odot E+\odot \odot$ \\
\hline
\end{tabular}

Nuclide [36] : Pa-234 S $6.70 \mathrm{~h}$

Halflife (Years) : 7.6484E-04

Inhalation 50-yr CEDE (Sv/Bq) : 4.1600E-10

Submersion $\quad(\mathrm{Sv}-\mathrm{m} 3) /(\mathrm{Bq}-\mathrm{sec}) \quad: 8.7200 \mathrm{E}-14$

Ground Shine $\quad(\mathrm{Sv}-\mathrm{m} 2) /(\mathrm{Bq}-\mathrm{sec}) \quad: 1.8000 \mathrm{E}-15$

Total Activity Released (Ci) : 7.9000E-08

Airborne Fraction : $1.0000 \mathrm{E}+00$

Respirable Fraction : $1.0000 \mathrm{E}+00$

Respirable Deposition Velocity $(\mathrm{cm} / \mathrm{sec}) \quad: 3.0000 \mathrm{E}-01$

Non-resp. Deposition Velocity $(\mathrm{cm} / \mathrm{sec})$ : $8.0000 \mathrm{E}+00$

\begin{tabular}{|c|c|c|}
\hline \multicolumn{3}{|c|}{ Nuclide [37] : U-234 S 2.445E5y } \\
\hline Halflife & (Years) & $2.4450 E+05$ \\
\hline Inhalation & CEDE $\quad(\mathrm{Sv} / \mathrm{Bq})$ & $9.4000 \mathrm{E}-06$ \\
\hline Submersion & $(\mathrm{Sv}-\mathrm{m} 3) /(\mathrm{Bq}-\mathrm{sec})$ & $6.1100 \mathrm{E}-18$ \\
\hline Ground Shine & $(\mathrm{Sv}-\mathrm{m} 2) /(\mathrm{Bq}-\mathrm{sec})$ & $5.8600 \mathrm{E}-19$ \\
\hline Total Activity Released & $(\mathrm{Ci})$ & $6.1000 \mathrm{E}-05$ \\
\hline Airborne Fraction & & 1. $.000 \mathrm{E}+0 \odot$ \\
\hline Respirable Fraction & & $1.0000 \mathrm{E}+\odot \odot$ \\
\hline Respirable Deposition V & $(\mathrm{cm} / \mathrm{sec})$ & $3.0000 \mathrm{E}-01$ \\
\hline Non-resp. Deposition V & $(\mathrm{cm} / \mathrm{sec})$ & $8 . \odot \odot \odot \odot E+\odot \odot$ \\
\hline
\end{tabular}

Nuclide [38] : Th-230 S 7.7E4y Halflife

(Years) : $\quad 7.7000 \mathrm{E}+04$ 


\begin{tabular}{|c|c|c|}
\hline Inhalation & $(\mathrm{Sv} / \mathrm{Bq})$ & $: 1.4000 \mathrm{E}-05$ \\
\hline Submersion & $(\mathrm{Sv}-\mathrm{m} 3) /(\mathrm{Bq}-\mathrm{sec})$ & 1. $4800 \mathrm{E}-17$ \\
\hline Ground Shine & $(\mathrm{Sv}-\mathrm{m} 2) /(\mathrm{Bq}-\mathrm{sec})$ & $6.3700 E-19$ \\
\hline Total Activity Released & (Ci) & $6.1000 \mathrm{E}-05$ \\
\hline Airborne Fraction & & 1. $\odot \odot \odot \odot E+\odot \odot$ \\
\hline Respirable Fraction & & 1. $\odot \odot \odot \odot E+\odot \odot$ \\
\hline Respirable Deposition & $(\mathrm{cm} / \mathrm{sec})$ & 3. $\odot \odot \odot \odot E-\odot 1$ \\
\hline Deposition V & Velocity & 8. $\odot \odot \odot \odot E+\odot \odot$ \\
\hline
\end{tabular}

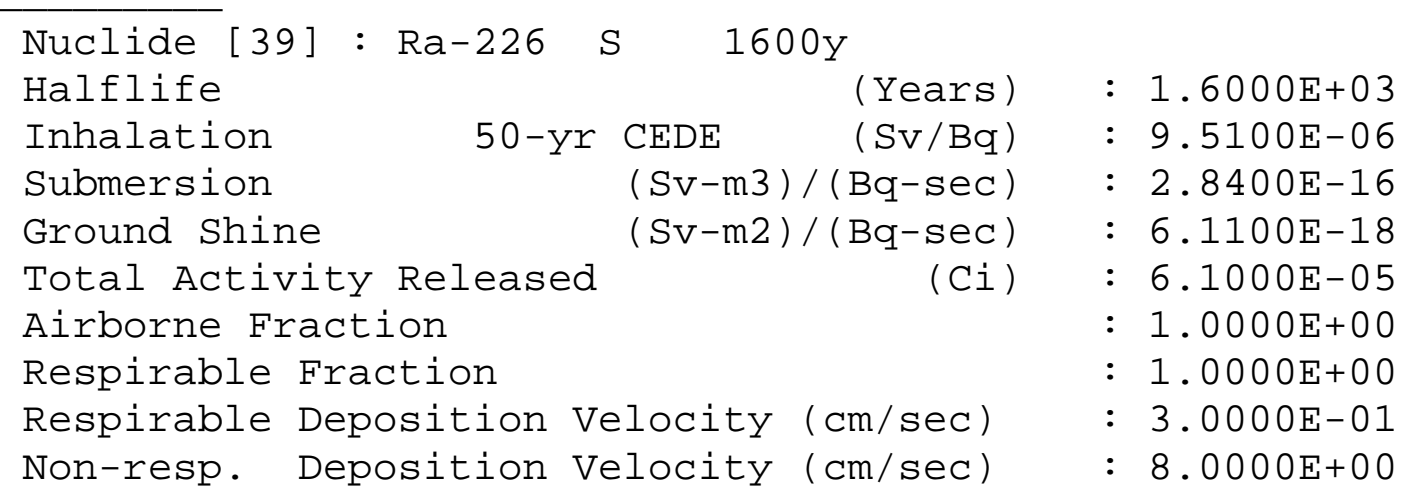

\begin{tabular}{|c|c|c|}
\hline $\begin{array}{l}\text { Nuclide [40] : Rn-222 } \\
\text { Halflife }\end{array}$ & $3.8235 d$ & $1.0475 E-02$ \\
\hline Inhalation & $(\mathrm{Sv} / \mathrm{Bq})$ & $\odot . \odot \odot \odot \odot E+\odot \odot$ \\
\hline Submersion & $(\mathrm{Sv}-\mathrm{m} 3) /(\mathrm{Bq}-\mathrm{sec})$ & $1.7700 \mathrm{E}-17$ \\
\hline Ground Shine & $(\mathrm{Sv}-\mathrm{m} 2) /(\mathrm{Bq}-\mathrm{sec})$ & 3.820 \\
\hline Total Activity Released & $(\mathrm{Ci})$ & $: 6$. \\
\hline Airborne Fraction & & $: 1.0$ \\
\hline Respirable Fraction & & $\partial \mathrm{E}+00$ \\
\hline Respirable Depositic & elocity ( $\mathrm{cm} / \mathrm{sec})$ & $: 0.0000 \mathrm{E}+\odot \odot$ \\
\hline Non-resp. Deposition & elocity $(\mathrm{cm} / \mathrm{sec})$ & $: 0.0000 \mathrm{E}+\odot \odot$ \\
\hline
\end{tabular}

\begin{tabular}{|c|c|c|}
\hline Nuclide [41] : Po-218 & $3.05 \mathrm{~m}$ & \\
\hline Halflife & (Years) & $5.8029 E-06$ \\
\hline Inhalation & CEDE $\quad(\mathrm{Sv} / \mathrm{Bq})$ & $\odot .0000 \mathrm{E}+0 \odot$ \\
\hline Submersion & $(\mathrm{Sv}-\mathrm{m} 3) /(\mathrm{Bq}-\mathrm{sec})$ & $4.2100 \mathrm{E}-19$ \\
\hline Ground Shine & $(\mathrm{Sv}-\mathrm{m} 2) /(\mathrm{Bq}-\mathrm{sec})$ & $8.6600 \mathrm{E}-21$ \\
\hline Total Activity Released & (Ci) & : $6.1000 \mathrm{E}-05$ \\
\hline Airborne Fraction & & $: 1.000$ \\
\hline Respirable Fraction & & : $1.0000 \mathrm{E}+0 \odot$ \\
\hline Respirable Deposition & $(\mathrm{cm} / \mathrm{sec})$ & : $3.0000 \mathrm{E}-01$ \\
\hline Non-resp. Deposition Ve & elocity ( $\mathrm{cm} / \mathrm{sec})$ & $: \quad 8.0000 \mathrm{E}+00$ \\
\hline
\end{tabular}




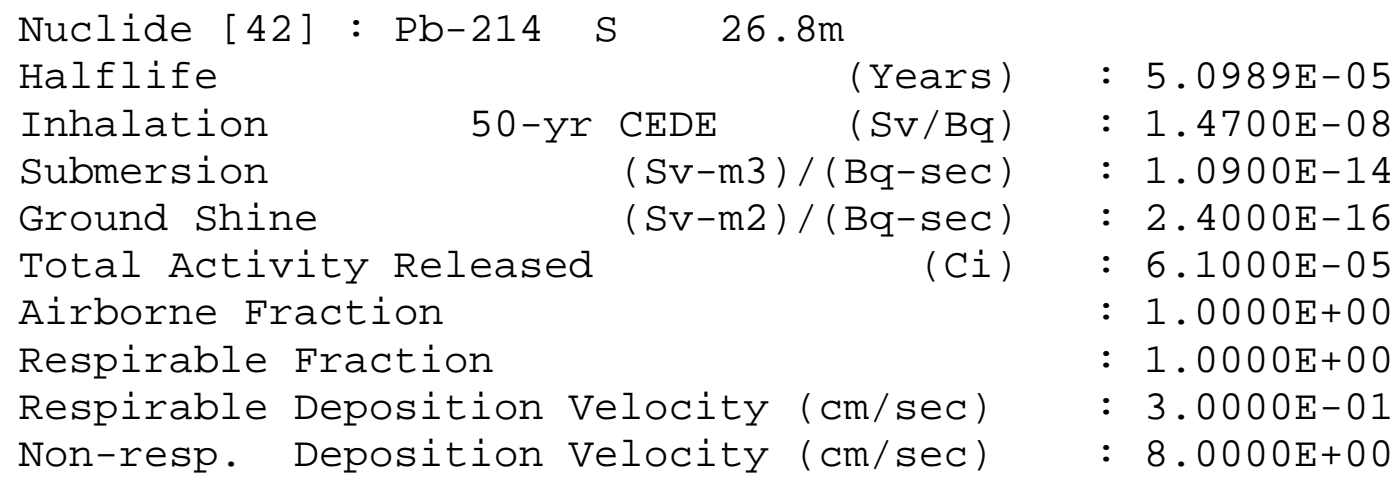

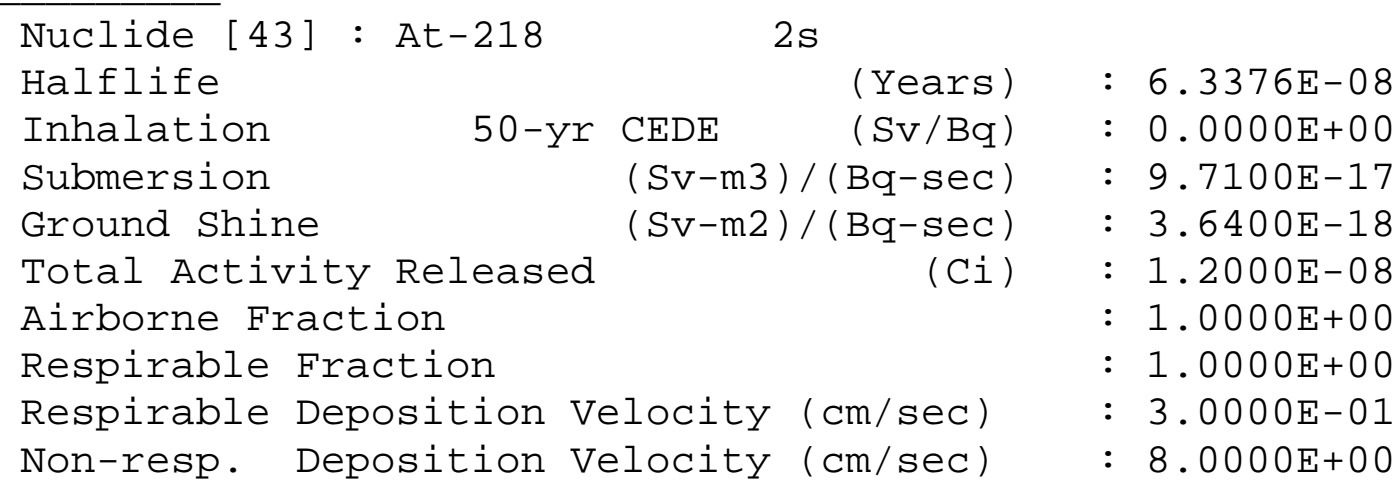

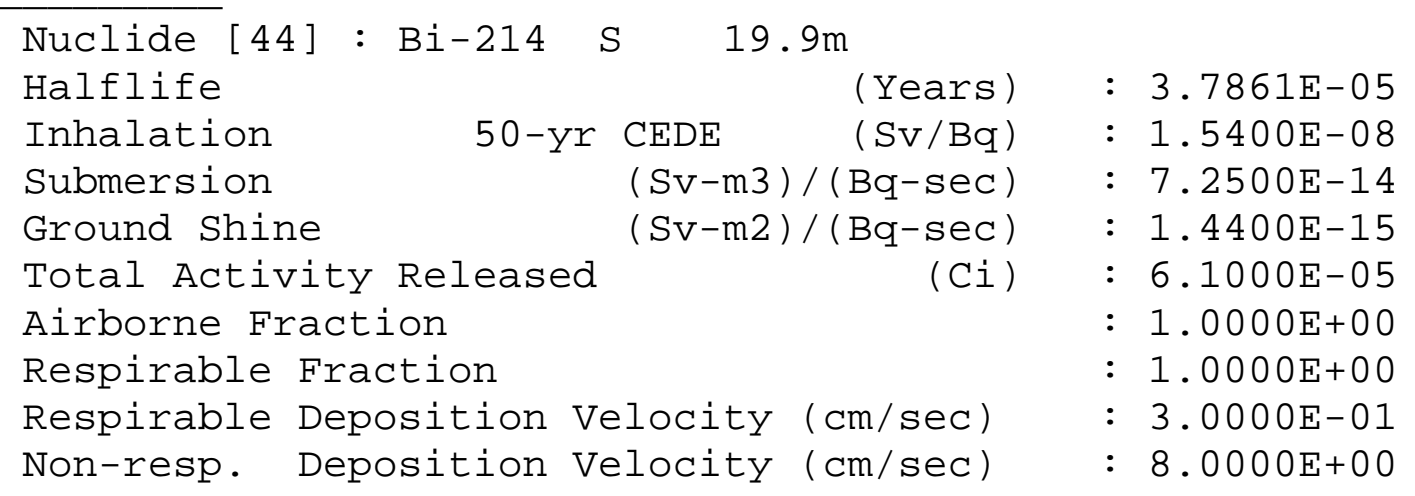

Nuclide [44] : Rn-220 55.6s

Halflife (Years) : $1.7619 \mathrm{E}-06$

Inhalation $\quad 50-y r$ CEDE $\quad(\mathrm{Sv} / \mathrm{Bq}) \quad: 0.0000 \mathrm{E}+00$

Submersion $\quad($ Sv-m3 $) /(B q-s e c): 1.7200 E-17$

Ground Shine $\quad(S v-m 2) /(B q-s e c): 3.6900 E-19$

Total Activity Released (Ci) : 5.4000E-05 
Airborne Fraction

Respirable Fraction

Respirable Deposition Velocity ( $\mathrm{cm} / \mathrm{sec}$ )

Non-resp. Deposition Velocity (cm/sec)
: $1.000 \odot \mathrm{E}+\odot \odot$

: $1.0000 \mathrm{E}+00$

: $\odot .0000 \mathrm{E}+0 \odot$

: $\odot . \odot \odot \odot \odot E+\odot \odot$
Nuclide [45] : Po-214 164.3u

Halflife

Inhalation

Submersion

Ground Shine

Total Activity Released

Airborne Fraction $\begin{array}{lll}50-y r \text { CEDE } & (\text { Years }) & : 1.6430 E+02 \\ (\text { Sv/Bq }) & : 0.000 \odot E+\odot \odot\end{array}$

$(\mathrm{Sv}-\mathrm{m} 3) /(\mathrm{Bq}-\mathrm{sec}) \quad: \quad 3.8100 \mathrm{E}-18$

$(\mathrm{Sv}-\mathrm{m} 2) /(\mathrm{Bq}-\mathrm{sec}): 7.9300 \mathrm{E}-20$

(Ci) : $6.1000 \mathrm{E}-05$

: $1.0000 \mathrm{E}+00$

: $1.0000 \mathrm{E}+00$

$\begin{array}{lll}\text { Respirable Deposition Velocity }(\mathrm{cm} / \mathrm{sec}) & : 3.0000 \mathrm{E}-01 \\ \text { Non-resp. Deposition Velocity }(\mathrm{cm} / \mathrm{sec}) & : 8.0000 \mathrm{E}+\odot \odot\end{array}$

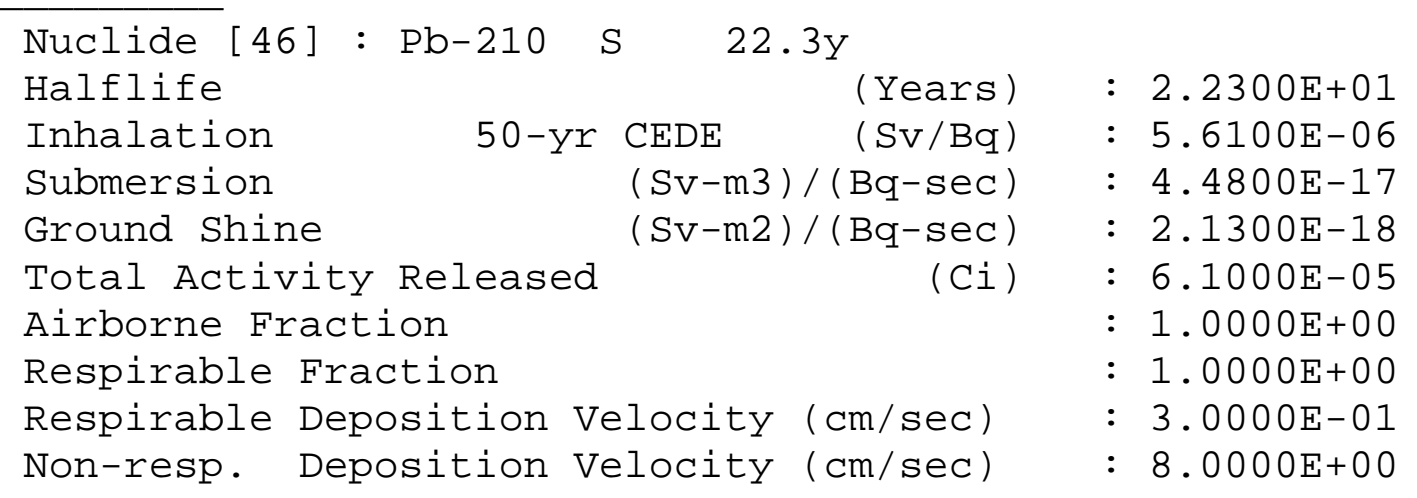

Nuclide [47] : Bi-210 S 5.012d

Halflife (Years) : 1.3732E-02

Inhalation $\quad 50-y r$ CEDE $\quad(\mathrm{Sv} / \mathrm{Bq}): 1.3300 \mathrm{E}-07$

Submersion $\quad(S v-m 3) /(B q-s e c): 2.5800 E-16$

Ground Shine $\quad(S v-m 2) /(B q-s e c): 3.5100 E-17$

Total Activity Released (Ci) : 6.1000E-05

Airborne Fraction $\quad$ : $1.0000 \mathrm{E}+00$

Respirable Fraction : $1.0000 \mathrm{E}+00$

Respirable Deposition Velocity (cm/sec) : $3.0000 \mathrm{c}-01$

Non-resp. Deposition Velocity (cm/sec) : 8.0000E+००

Nuclide [48] : Po-210 S 138.38d

Halflife (Years) : 3.7912E-01 
Appendix C

\begin{tabular}{|c|c|c|}
\hline Inhalation & $(\mathrm{Sv} / \mathrm{Bq})$ & $4.2700 \mathrm{E}-\odot 6$ \\
\hline Submersion & $(\mathrm{Sv}-\mathrm{m} 3) /(\mathrm{Bq}-\mathrm{sec})$ & 3. $8900 \mathrm{E}-19$ \\
\hline Ground Shine & $(\mathrm{Sv}-\mathrm{m} 2) /(\mathrm{Bq}-\mathrm{sec})$ & $8.0900 E-21$ \\
\hline Total Activity Released & (Ci) & $6.1000 \mathrm{E}-05$ \\
\hline Airborne Fraction & & 1. $.000 \mathrm{E}+00$ \\
\hline Respirable Fraction & & 1. $.000 \mathrm{E}+\odot \odot$ \\
\hline Respirable & $(\mathrm{cm} / \mathrm{sec})$ & $3.0000 \mathrm{E}-01$ \\
\hline Deposition & $(\mathrm{cm} / \mathrm{sec})$ & ८. ๑๑९९Е+๑९ \\
\hline
\end{tabular}




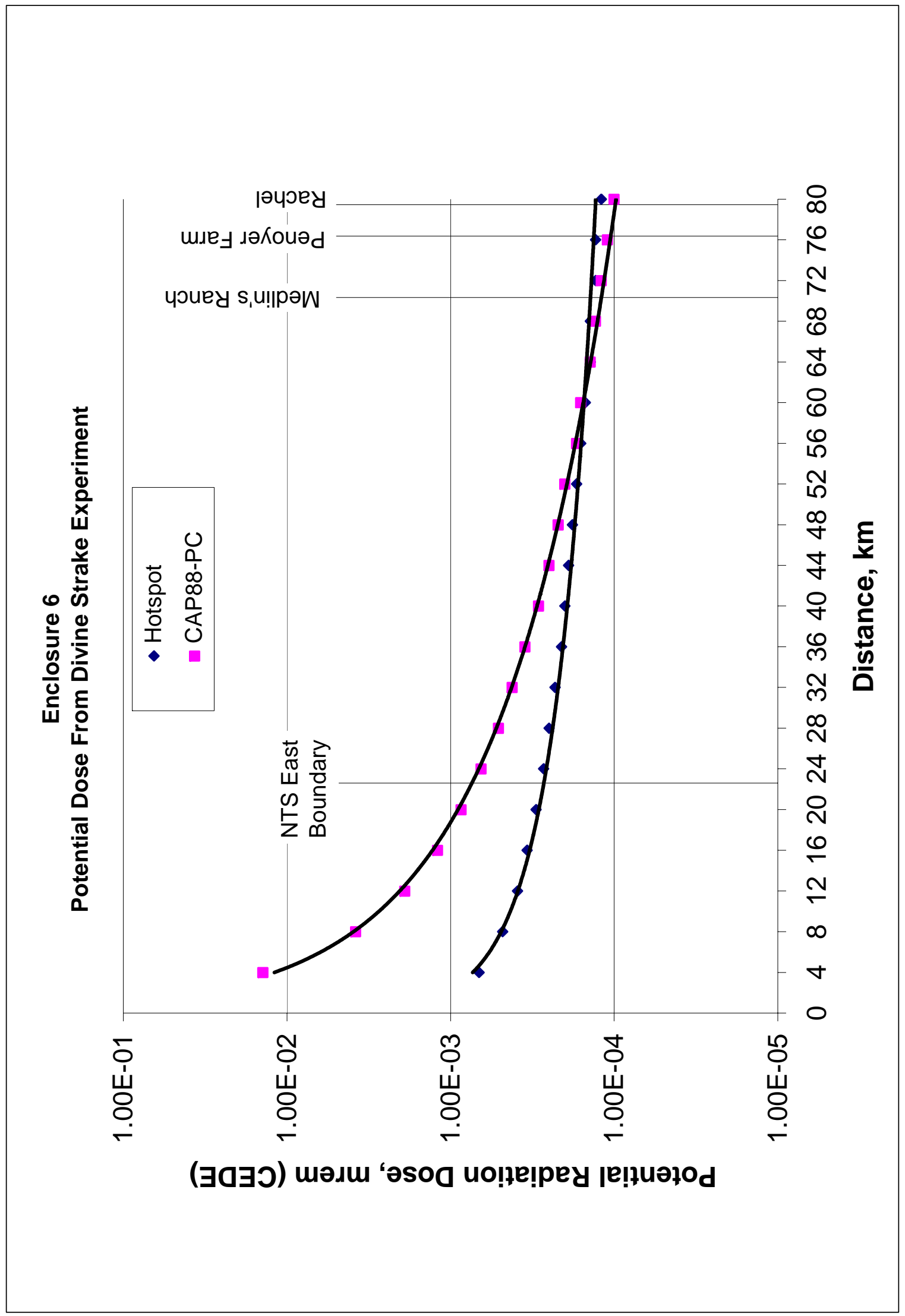

C - 41 
Appendix C

THIS PAGE INTENTIONALLY LEFT BLANK 


\section{Appendix D}

\section{Output from CAP88-PC Version 3.0 Modtest Sample Case Run October 2006}

Prior to modeling potential dose from the Divine Strake experiment the Modtest sample case, described in the CAP88-PC Version 3.0 Users Guide, was run on the same computer and software installation as was used for dose estimations from the Divine Strake experiment. Results of this test were identical to those from the published example (Shroff, 2006). The Synopsis and Dose and Risk Equivalent Summary Reports from our run of Modtest are provided in this Appendix for comparison with those given in Appendix G of the CAP88-PC Version 3.0 Users Guide (Shroff, 2006). 


\author{
C A P $88-P$ C \\ Version 3.0 \\ Clean Air Act Assessment Package - 1988

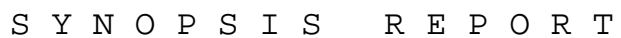 \\ Non-Radon Population Assessment \\ Oct 12, $2006 \quad 09: 29 \mathrm{am}$
}

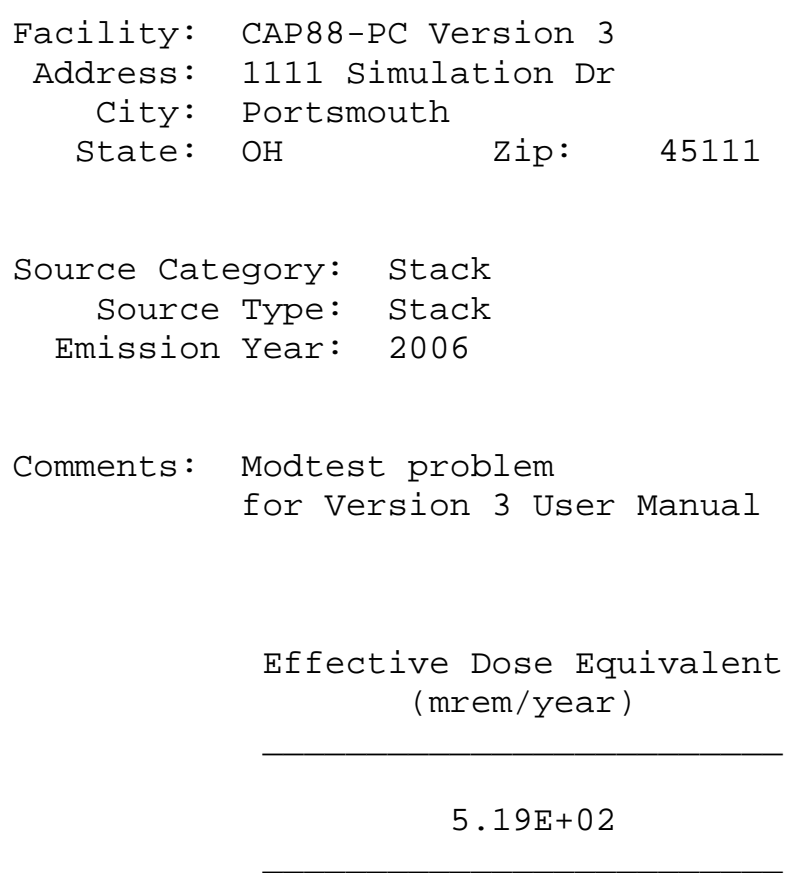

At This Location: 805 Meters East Northeast

Dataset Name: ModelTest 0ct16

Dataset Date: 10/12/2006 8:48:00 AM

Wind File: C: \Program Files \CAP88-PC30\WindLib\P0RTS30.

Population File: C: \Program Files \CAP88-PC30 \Poplib\P0RTS.POP 
$\begin{array}{lll}\text { 0ct } 12,2006 & 09: 29 \text { am } & \text { SYNOPSIS } \\ \text { Page } 1\end{array}$

MAXIMALLY EXPOSED INDIVIDUAL

Location of The Individual: 805 Meters East Northeast Lifetime Fatal Cancer Risk: 4.13E-04

ORGAN DOSE EQUIVALENT SUMMARY

\begin{tabular}{|c|c|c|}
\hline Organ & $\begin{array}{c}\text { Selected } \\
\text { Individual } \\
\text { (mrem/y) }\end{array}$ & $\begin{array}{c}\text { Collective } \\
\text { Population } \\
\text { (person-rem/y) }\end{array}$ \\
\hline Adrenals & 1. $70 \mathrm{E}+01$ & $2.99 E+01$ \\
\hline B Surfac & $1.82 E+01$ & $3.21 E+01$ \\
\hline Breasts & $8.43 E+01$ & $1.47 E+02$ \\
\hline St Wall & $1.75 \mathrm{E}+01$ & 3. $08 E+01$ \\
\hline ULI Wall & $2.28 E+01$ & $4.02 E+01$ \\
\hline Kidneys & $1.56 E+01$ & $2.76 \mathrm{E}+01$ \\
\hline Lungs & $1.82 E+01$ & $3.22 E+01$ \\
\hline Ovaries & $1.71 E+01$ & 3. $03 E+01$ \\
\hline R Marrow & 1. $79 \mathrm{E}+01$ & $3.19 E+01$ \\
\hline Spleen & $1.85 E+01$ & $3.34 \mathrm{E}+01$ \\
\hline Thymus & 3. 19E+01 & $5.54 \mathrm{E}+01$ \\
\hline Uterus & $2.24 \mathrm{E}+01$ & 3. 93E+01 \\
\hline Bld Wall & $2.03 E+02$ & $2.57 E+02$ \\
\hline Brain & $2.18 E+01$ & $3.85 E+01$ \\
\hline Esophagu & 1. 71E+०1 & $3.02 E+01$ \\
\hline SI Wall & $1.65 E+01$ & $2.92 E+01$ \\
\hline LLI Wall & $2.17 E+01$ & $3.82 E+01$ \\
\hline Liver & $7.69 E+02$ & $1.36 E+03$ \\
\hline Muscle & $1.83 E+01$ & $3.24 \mathrm{E}+01$ \\
\hline Pancreas & $2.26 E+01$ & 3. 99E+01 \\
\hline Skin & $1.77 E+01$ & $3.12 E+01$ \\
\hline Testes & $1.97 E+01$ & $3.47 E+01$ \\
\hline Thyroid & $1.72 E+01$ & $3.03 E+01$ \\
\hline EFFEC & $5.19 E+02$ & $6.68 E+02$ \\
\hline
\end{tabular}

FREQUENCY DISTRIBUTION OF LIFETIME FATAL CANCER RISKS

\section{Risk Range}

1. $\odot E+\odot \odot$ TO 1. $0 \mathrm{E}-01$

1. $0 \mathrm{E}-01$ TO 1. $0 \mathrm{E}-02$

1. $\mathrm{OE}-02$ TO 1. $\mathrm{OE}-03$

1. $\odot \mathrm{E}-\odot 3$ TO 1. $\mathrm{OE}-\odot 4$

1. 0 - $\odot 4$ TO 1. 0 E- $\odot 5$

1. OE-05 TO 1.0E-06

LESS THAN 1. 0 - - 6

\begin{abstract}
\# of People
Deaths/Year in This

Deaths/Year

\# of in This Risk

People Range or Higher Risk Range Range or Higher

in This Risk
\end{abstract}

$\begin{array}{rr}0 & 0 \\ 0 & 0 \\ 0 & 0 \\ 194 & 194 \\ 4566 & 4760 \\ 121146 & 125906 \\ 536315 & 662221\end{array}$

$\odot . \odot \odot E+\odot \odot$
$\odot . \odot \odot E+\odot \odot$
$\odot . \odot \odot E+\odot \odot$
$6 . \odot 8 E-\odot 4$
$1.31 E-\odot 3$
$3 . \odot 2 E-\odot 3$
$1.73 E-\odot 3$

$\odot . ~ . \odot E+\odot \odot$

$\odot .00 \mathrm{E}+\odot \odot$

$\odot ., \odot E+\odot \odot$

$6.08 E-04$

1. $92 \mathrm{E}-03$

4. $94 \mathrm{E}-03$

$6.67 \mathrm{E}-03$ 


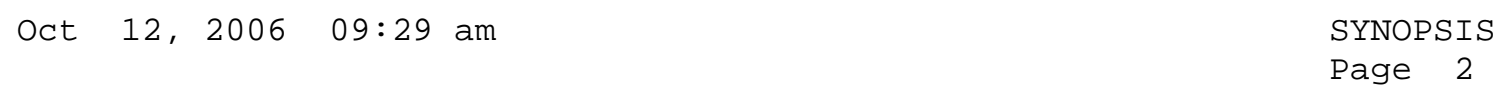

RADIONUCLIDE EMISSIONS DURING THE YEAR 2006

Source

\begin{tabular}{|c|c|c|c|c|}
\hline Nuclide & Type & Size & $\begin{array}{c}\# 1 \\
\mathrm{Ci} / \mathrm{y}\end{array}$ & $\begin{array}{r}\text { TOTAL } \\
\mathrm{Ci} / \mathrm{y}\end{array}$ \\
\hline$U-238$ & M & 1 & 1. $\mathrm{OE}+\Theta 1$ & 1. $\mathrm{OE}+01$ \\
\hline U-235 & M & 1 & 1. $\odot E+\Theta 1$ & 1. $0 \mathrm{E}+01$ \\
\hline$U-234$ & M & 1 & $8 . \odot E+\odot \odot$ & 8. $\odot E+\odot \odot$ \\
\hline
\end{tabular}

\section{SITE INFORMATION}

Temperature: 10 degrees $\mathrm{C}$ Precipitation: $\quad 100 \mathrm{~cm} / \mathrm{y}$ Humidity: $\quad 8 \mathrm{~g} / \mathrm{cu} \mathrm{m}$ Mixing Height: $1000 \mathrm{~m}$ 


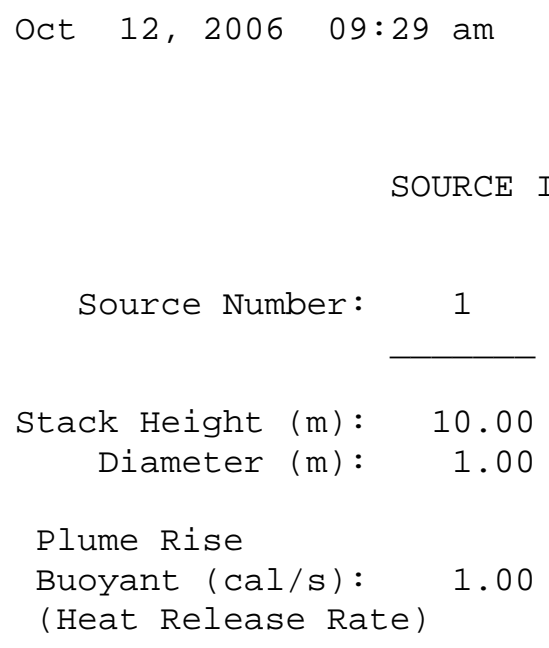

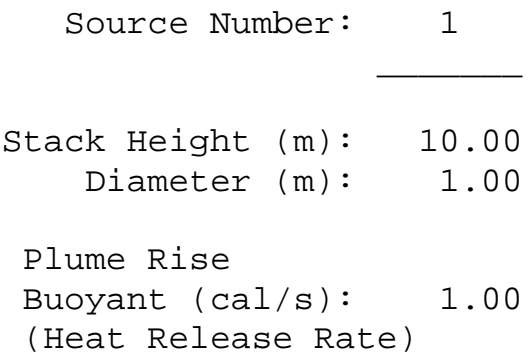

\section{SOURCE INFORMATION}

AGRICULTURAL DATA

\begin{tabular}{|c|c|c|c|}
\hline & Vegetable & Milk & Meat \\
\hline Fraction Home Produced: & $\odot .70 \odot$ & 0.400 & 0.440 \\
\hline Fraction From Assessment Area: & 0.300 & 0.600 & 0.560 \\
\hline Fraction Imported: & 0.000 & 0.000 & 0.000 \\
\hline
\end{tabular}

$\begin{array}{cc}\text { Beef Cattle Density: } & 2.03 \mathrm{E}-\odot 1 \\ \text { Milk Cattle Density: } & 4.56 \mathrm{E}-\odot 2 \\ \text { Land Fraction Cultivated } & \\ \text { for Vegetable Crops: } & 1.70 \mathrm{E}-\odot 2\end{array}$


oct 12, $2006 \quad 09: 29$ am

SYNOPSIS

Page 4

POPULATION DATA

\begin{tabular}{|c|c|c|c|c|c|c|c|}
\hline \multirow[b]{2}{*}{ Direction } & \multicolumn{6}{|c|}{ Distance $(\mathrm{m})$} & \multirow[b]{2}{*}{24150} \\
\hline & 805 & 2415 & 4025 & 5635 & 7245 & 12075 & \\
\hline $\mathrm{N}$ & $\odot$ & $\odot$ & 19 & 443 & 803 & 3785 & 0 \\
\hline NNW & $\odot$ & 9 & 14 & 10 & 34 & 1069 & 3248 \\
\hline NW & $\odot$ & 121 & $\odot$ & 371 & 141 & 1106 & 2284 \\
\hline WNW & 0 & 0 & $\odot$ & 0 & 33 & 922 & 1600 \\
\hline W & 30 & $\odot$ & $4 \odot$ & 57 & 46 & 876 & 1348 \\
\hline WSW & 57 & 6 & 9 & 16 & 20 & 569 & 1674 \\
\hline SW & 46 & 7 & 57 & 224 & 20 & 707 & 1375 \\
\hline SSW & 38 & 0 & 173 & 40 & 71 & 1631 & 3183 \\
\hline$S$ & 7 & 18 & 207 & 144 & 36 & 3518 & 30593 \\
\hline SSE & $\Theta$ & 104 & 18 & 35 & 170 & 1656 & 13613 \\
\hline SE & 7 & 39 & 10 & 3 & 75 & 986 & 4587 \\
\hline ESE & 2 & 12 & 5 & 57 & 63 & 878 & 1980 \\
\hline$E$ & 6 & 54 & 40 & 2 & 96 & 1102 & 5808 \\
\hline ENE & 1 & 65 & 37 & 93 & 95 & 1023 & 2435 \\
\hline NE & 0 & 12 & 20 & 63 & 225 & 359 & 2329 \\
\hline NNE & $\odot$ & 10 & 82 & 79 & 567 & 2780 & 2266 \\
\hline
\end{tabular}

Distance $(\mathrm{m})$

\begin{tabular}{rrrr} 
& & & \\
\cline { 3 - 4 } Direction & 40250 & 56350 & 72200 \\
& & & \\
& & & \\
$N$ & 42304 & 7518 & 26978 \\
NNW & 4628 & 4028 & 21176 \\
NW & 4111 & 12150 & 7605 \\
WNW & 6021 & 13838 & 9880 \\
W & 5591 & 7376 & 18285 \\
WSW & 2464 & 11058 & 17205 \\
SW & 1923 & 2702 & 5657 \\
SSW & 3732 & 6222 & 4633 \\
S & 4489 & 3037 & 14068 \\
SSE & 14145 & 43111 & 76266 \\
SE & 4108 & 4698 & 14064 \\
ESE & 6106 & 5645 & 25178 \\
E & 7400 & 4997 & 8015 \\
ENE & 11823 & 5583 & 9245 \\
NE & 2653 & 3232 & 16780 \\
NNE & 3879 & 7594 & 12216
\end{tabular}

D - 6 


\author{
C A P $88-P$ C \\ Version 3.0 \\ Clean Air Act Assessment Package - 1988
}
D O S E A N D R I S K E Q U I V A L E N T S U M M A R I E S
Non-Radon Population Assessment
Oct 12, $2006 \quad 09: 29$ am

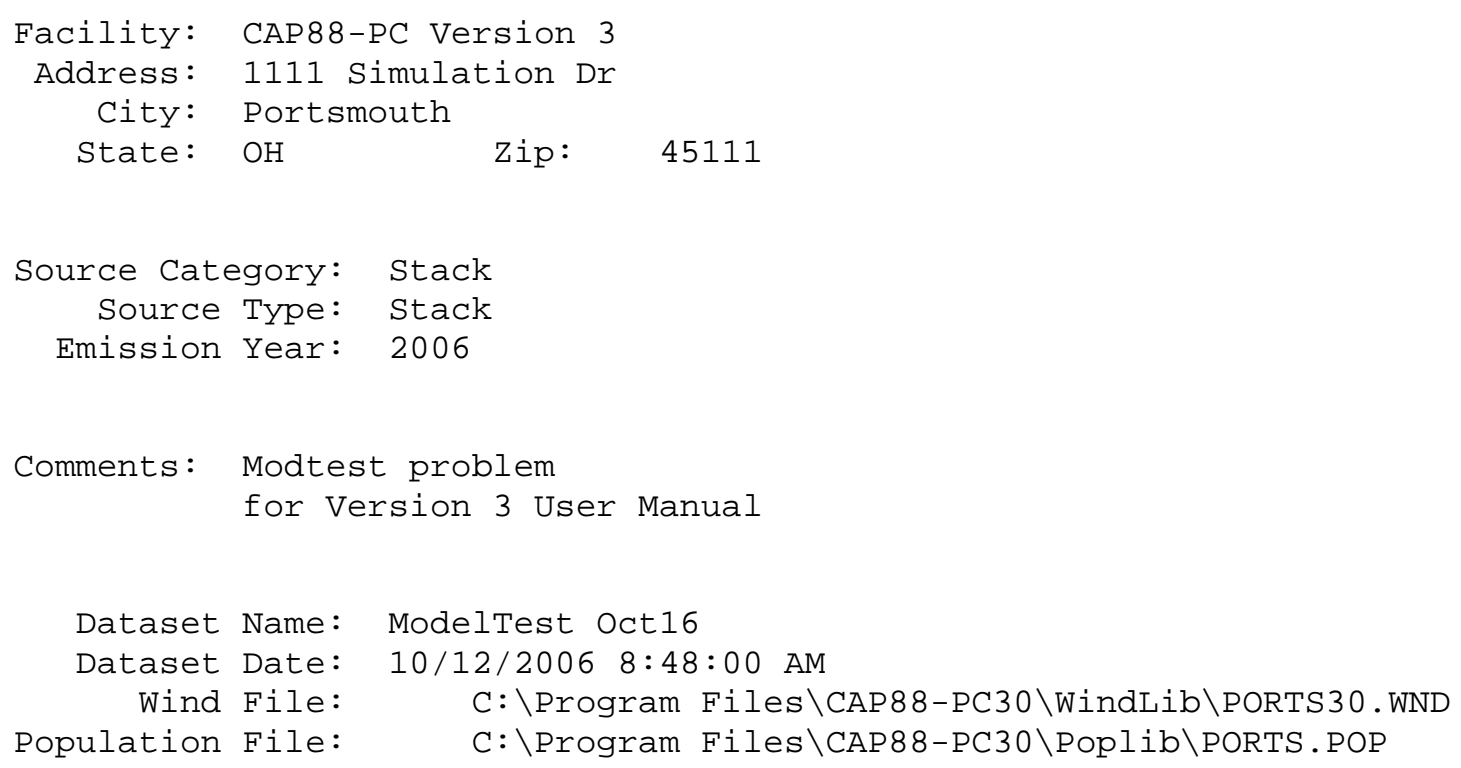




\begin{tabular}{|c|c|c|}
\hline Organ & $\begin{array}{l}\text { Selected } \\
\text { Individual } \\
(\mathrm{mrem} / \mathrm{y})\end{array}$ & $\begin{array}{c}\text { Collective } \\
\text { Population } \\
\text { (person-rem/y) }\end{array}$ \\
\hline Adrenals & $1.7 \odot \mathrm{E}+\odot 1$ & $2.99 \mathrm{E}+01$ \\
\hline B Surfac & $1.82 \mathrm{E}+01$ & $3.21 E+01$ \\
\hline Breasts & $8.43 E+01$ & $1.47 \mathrm{E}+02$ \\
\hline St Wall & $1.75 \mathrm{E}+01$ & $3.08 \mathrm{E}+01$ \\
\hline ULI Wall & $2.28 \mathrm{E}+01$ & $4.02 \mathrm{E}+01$ \\
\hline Kidneys & $1.56 \mathrm{E}+01$ & $2.76 \mathrm{E}+01$ \\
\hline Lungs & $1.82 \mathrm{E}+01$ & $3.22 \mathrm{E}+01$ \\
\hline ovaries & $1.71 \mathrm{E}+01$ & $3.03 E+01$ \\
\hline R Marrow & $1.79 \mathrm{E}+01$ & $3.19 \mathrm{E}+01$ \\
\hline Spleen & $1.85 \mathrm{E}+01$ & $3.34 \mathrm{E}+01$ \\
\hline Thymus & $3.19 \mathrm{E}+01$ & $5.54 \mathrm{E}+01$ \\
\hline Uterus & $2.24 \mathrm{E}+01$ & $3.93 \mathrm{E}+01$ \\
\hline Bld Wall & $2.03 \mathrm{E}+\odot 2$ & $2.57 \mathrm{E}+02$ \\
\hline Brain & $2.18 \mathrm{E}+01$ & $3.85 \mathrm{E}+01$ \\
\hline Esophagu & $1.71 \mathrm{E}+01$ & $3.02 \mathrm{E}+01$ \\
\hline SI Wall & $1.65 E+01$ & $2.92 \mathrm{E}+01$ \\
\hline LLI Wall & $2.17 \mathrm{E}+01$ & $3.82 \mathrm{E}+01$ \\
\hline Liver & $7.69 \mathrm{E}+02$ & $1.36 \mathrm{E}+03$ \\
\hline Muscle & $1.83 \mathrm{E}+01$ & $3.24 \mathrm{E}+01$ \\
\hline Pancreas & $2.26 \mathrm{E}+01$ & $3.99 \mathrm{E}+01$ \\
\hline Skin & $1.77 \mathrm{E}+01$ & $3.12 \mathrm{E}+01$ \\
\hline Testes & $1.97 \mathrm{E}+01$ & $3.47 \mathrm{E}+01$ \\
\hline Thyroid & $1.72 \mathrm{E}+01$ & $3.03 \mathrm{E}+01$ \\
\hline EFFEC & $5.19 \mathrm{E}+02$ & $6.68 \mathrm{E}+02$ \\
\hline
\end{tabular}

PATHWAY EFFECTIVE DOSE EQUIVALENT SUMMARY

Pathway

INGESTION
INHALATION
AIR IMMERSION
GROUND SURFACE
INTERNAL
EXTERNAL

TOTAL
Selected Individual (mrem/y)

$1.74 \mathrm{E}+01$

$4.75 \mathrm{E}+02$

$1.39 \mathrm{E}-03$

2. $58 \mathrm{E}+01$

4. $93 \mathrm{E}+02$

2. $58 \mathrm{E}+01$

$5 \cdot 19 \mathrm{E}+02$
Collective Population (person-rem/y)
4. $73 \mathrm{E}+01$
$5.75 \mathrm{E}+02$
1. $68 \mathrm{E}-03$
4. $58 \mathrm{E}+01$
6. $23 \mathrm{E}+\mathrm{O} 2$
4. $58 \mathrm{E}+01$
$6.68 \mathrm{E}+02$ 
Oct 12, $200609: 29$ am SUMMARY

NUCLIDE EFFECTIVE DOSE EQUIVALENT SUMMARY

\begin{tabular}{|c|c|c|}
\hline Nuclides & $\begin{array}{l}\text { Selected } \\
\text { Individual } \\
(\mathrm{mrem} / \mathrm{y})\end{array}$ & $\begin{array}{c}\text { Collective } \\
\text { Population } \\
\text { (person-rem/y) }\end{array}$ \\
\hline U-238 & $1.62 \mathrm{E}+02$ & $2 . \odot 4 \mathrm{E}+\odot 2$ \\
\hline Th-234 & 7. 34E- 01 & $1.42 \mathrm{E}+\odot \odot$ \\
\hline $\mathrm{Pa}-234 \mathrm{~m}$ & $8.45 \mathrm{E}+\odot \odot$ & $1.5 \odot \mathrm{E}+\odot 1$ \\
\hline $\mathrm{Pa}-234$ & $4.64 \mathrm{E}-01$ & $8.21 E-01$ \\
\hline U-234 & $1.57 \mathrm{E}+\odot 2$ & $1.98 \mathrm{E}+02$ \\
\hline Th-230 & $3.99 E-05$ & $1.03 E-04$ \\
\hline $\mathrm{Ra}-226$ & $7.76 \mathrm{E}-27$ & $2.13 E-26$ \\
\hline $\mathrm{Rn}-222$ & $\odot . \odot \odot E+\odot \odot$ & $\odot . \odot \odot \mathrm{E}+\odot \odot$ \\
\hline Po- 218 & $7.96 \mathrm{E}-32$ & 1. $41 \mathrm{E}-31$ \\
\hline $\mathrm{Pb}-214$ & $2.21 \mathrm{E}-27$ & $3.91 \mathrm{E}-27$ \\
\hline $\mathrm{Bi}-214$ & $1.33 E-26$ & $2.35 E-26$ \\
\hline Po-214 & $7.28 \mathrm{E}-31$ & $1.29 \mathrm{E}-30$ \\
\hline $\mathrm{Pb}-210$ & $2.85 \mathrm{E}-27$ & $7.67 \mathrm{E}-27$ \\
\hline $\mathrm{Bi}-210$ & 1. $10 \mathrm{E}-28$ & $1.98 \mathrm{E}-28$ \\
\hline Po- 210 & $9.35 E-28$ & $2.73 E-27$ \\
\hline At -218 & $\odot . \odot \odot \mathrm{E}+\odot \odot$ & $\odot . \odot \odot \mathrm{E}+\odot \odot$ \\
\hline U-235 & $1.89 E+02$ & $2.46 \mathrm{E}+\odot 2$ \\
\hline Th-231 & $1.62 \mathrm{E}+00$ & $2.86 \mathrm{E}+00$ \\
\hline $\mathrm{Pa}-231$ & $3.55 E-\odot 4$ & $9.03 E-\odot 4$ \\
\hline$A C-227$ & $6.35 E-07$ & $1.64 \mathrm{E}-06$ \\
\hline Th- 227 & 1. $\odot 8 E-\odot 7$ & $1.94 \mathrm{E}-07$ \\
\hline $\mathrm{Ra}-223$ & $1.65 \mathrm{E}-07$ & $3.55 E-07$ \\
\hline$R n-219$ & $\odot . \odot \odot E+\odot \odot$ & $\odot . \odot \odot E+\odot \odot$ \\
\hline Po- 215 & $1.26 \mathrm{E}-10$ & $2.22 \mathrm{E}-10$ \\
\hline $\mathrm{Pb}-211$ & 7. . $99 \mathrm{E}-\odot 8$ & $1.26 \mathrm{E}-07$ \\
\hline $\mathrm{Bi}-211$ & $3.29 \mathrm{E}-\odot 8$ & $5.82 \mathrm{E}-08$ \\
\hline $\mathrm{Tl}-207$ & $4.14 \mathrm{E}-\odot 8$ & $7.34 \mathrm{E}-08$ \\
\hline Po-211 & $4.55 E-29$ & $8.06 \mathrm{E}-29$ \\
\hline $\mathrm{Fr}-223$ & $2.62 \mathrm{E}-27$ & $4.64 \mathrm{E}-27$ \\
\hline TOTAL & $5.19 \mathrm{E}+\odot 2$ & $6.68 \mathrm{E}+02$ \\
\hline
\end{tabular}




\begin{tabular}{lcc} 
Cancer & $\begin{array}{c}\text { Selected Individual } \\
\text { Total Lifetime } \\
\text { Fatal Cancer Risk }\end{array}$ & $\begin{array}{c}\text { Total Collective } \\
\text { Population Fatal } \\
\text { Cancer Risk } \\
\text { (Deaths/y) }\end{array}$ \\
\cline { 2 - 3 } Stomach & $3.73 \mathrm{E}-07$ & \\
Colon & $1.21 \mathrm{E}-06$ & $8.48 \mathrm{E}-06$ \\
Liver & $5.18 \mathrm{E}-06$ & $2.81 \mathrm{E}-05$ \\
LUNG & $1.69 \mathrm{E}-06$ & $1.45 \mathrm{E}-04$ \\
Bone & $3.93 \mathrm{E}-04$ & $3.84 \mathrm{E}-05$ \\
Skin & $9.52 \mathrm{E}-07$ & $6.18 \mathrm{E}-03$ \\
Breast & $7.81 \mathrm{E}-07$ & $2.16 \mathrm{E}-05$ \\
Ovary & $1.31 \mathrm{E}-06$ & $1.79 \mathrm{E}-05$ \\
Bladder & $4.71 \mathrm{E}-07$ & $3.01 \mathrm{E}-05$ \\
Kidneys & $9.05 \mathrm{E}-07$ & $1.07 \mathrm{E}-05$ \\
Thyroid & $1.73 \mathrm{E}-06$ & $2.07 \mathrm{E}-05$ \\
Leukemia & $9.86 \mathrm{E}-08$ & $3.93 \mathrm{E}-05$ \\
Residual & $1.32 \mathrm{E}-06$ & $2.26 \mathrm{E}-06$ \\
Total & $4.30 \mathrm{E}-06$ & $3.00 \mathrm{E}-05$ \\
& $4.13 \mathrm{E}-04$ & $9.89 \mathrm{E}-05$ \\
& & $6.67 \mathrm{E}-03$
\end{tabular}

PATHWAY RISK SUMMARY

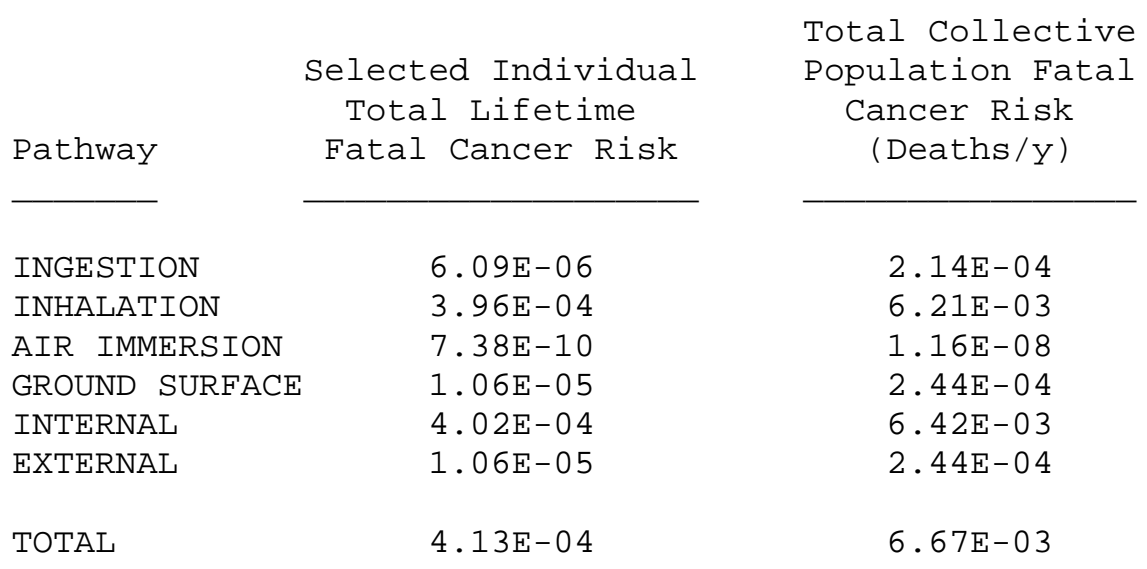


NUCLIDE RISK SUMMARY

\begin{tabular}{|c|c|c|}
\hline Nuclide & $\begin{array}{l}\text { Selected Individual } \\
\text { Total Lifetime } \\
\text { Fatal Cancer Risk }\end{array}$ & $\begin{array}{c}\text { Total Collective } \\
\text { Population Fatal } \\
\text { Cancer Risk } \\
\text { (Deaths/y) }\end{array}$ \\
\hline$U-238$ & $1.32 \mathrm{E}-04$ & $2.10 \mathrm{E}-03$ \\
\hline Th-234 & $5.20 E-\odot 7$ & $1.42 \mathrm{E}-05$ \\
\hline $\mathrm{Pa}-234 \mathrm{~m}$ & $1.35 \mathrm{E}-06$ & $3.10 \mathrm{E}-05$ \\
\hline $\mathrm{Pa}-234$ & $2.53 E-07$ & $5.80 E-06$ \\
\hline U-234 & $1.28 \mathrm{E}-\odot 4$ & $2.04 \mathrm{E}-03$ \\
\hline Th-230 & $4.10 \mathrm{E}-12$ & $1.37 \mathrm{E}-10$ \\
\hline $\mathrm{Ra}-226$ & $2.66 \mathrm{E}-33$ & $9.48 E-32$ \\
\hline$R n-222$ & $\odot . \odot \odot E+\odot \odot$ & $\odot . \odot \odot E+\odot \odot$ \\
\hline Po- 218 & $4.36 E-38$ & 1. $00 \mathrm{E}-36$ \\
\hline $\mathrm{Pb}-214$ & $1.18 \mathrm{E}-33$ & $2.70 \mathrm{E}-32$ \\
\hline $\mathrm{Bi}-214$ & $7.04 \mathrm{E}-33$ & $1.62 \mathrm{E}-31$ \\
\hline Po-214 & $4.00 \mathrm{E}-37$ & $9.17 \mathrm{E}-36$ \\
\hline $\mathrm{Pb}-210$ & $9.45 \mathrm{E}-34$ & $3.30 \mathrm{E}-32$ \\
\hline Bi-210 & $1.58 \mathrm{E}-35$ & $4.07 E-34$ \\
\hline Po-210 & $3.58 \mathrm{E}-34$ & $1.35 E-32$ \\
\hline At -218 & $\odot . \odot \odot \mathrm{E}+\odot \odot$ & $\odot . \odot \odot \mathrm{E}+\odot \odot$ \\
\hline U-235 & $1.50 \mathrm{E}-\odot 4$ & $2.45 \mathrm{E}-03$ \\
\hline Th-231 & $7.30 \mathrm{E}-07$ & $1.67 \mathrm{E}-05$ \\
\hline $\mathrm{Pa}-231$ & $3.95 \mathrm{E}-11$ & $1.22 \mathrm{E}-09$ \\
\hline Ac -227 & $1.05 \mathrm{E}-13$ & $3.52 \mathrm{E}-12$ \\
\hline Th - 227 & $6.09 \mathrm{E}-14$ & $1.45 \mathrm{E}-12$ \\
\hline $\mathrm{Ra}-223$ & $8.93 E-14$ & $2.49 \mathrm{E}-12$ \\
\hline $\mathrm{Rn}-219$ & $\odot . \odot \odot E+\odot \odot$ & $\odot . \odot \odot E+\odot \odot$ \\
\hline Po- 215 & $6.88 \mathrm{E}-17$ & $1.58 \mathrm{E}-15$ \\
\hline $\mathrm{Pb}-211$ & $2.35 \mathrm{E}-14$ & $5.40 \mathrm{E}-13$ \\
\hline Bi-211 & $1.80 \mathrm{E}-14$ & $4.13 E-13$ \\
\hline Tl-207 & $5.29 \mathrm{E}-15$ & $1.21 \mathrm{E}-13$ \\
\hline Po-211 & $2.49 \mathrm{E}-35$ & $5.72 \mathrm{E}-34$ \\
\hline $\mathrm{Fr}-223$ & $9.93 \mathrm{E}-34$ & $2.28 \mathrm{E}-32$ \\
\hline TOTAL & $4.13 E-\odot 4$ & $6.67 \mathrm{E}-03$ \\
\hline
\end{tabular}


Oct 12, $2006 \quad 09: 29$ am

SUMMARY

Page 5

INDIVIDUAL EFFECTIVE DOSE EQUIVALENT RATE (mrem/y)

(All Radionuclides and Pathways)

\begin{tabular}{|c|c|c|c|c|c|c|c|}
\hline \multirow[b]{2}{*}{ Direction } & \multicolumn{7}{|c|}{ Distance (m) } \\
\hline & 805 & 2415 & 4025 & 5635 & 7245 & 12075 & 24150 \\
\hline $\mathrm{N}$ & $\odot . \odot \mathrm{O}+\odot \odot$ & $\odot . \odot \mathrm{O}+\odot \odot$ & $5.5 \mathrm{E}+01$ & $3.3 E+01$ & $2.2 \mathrm{E}+01$ & $9.8 \mathrm{E}+00$ & $\odot . \odot \mathrm{E}+\odot \odot$ \\
\hline NNW & $\odot . \odot \mathrm{O}+\odot \odot$ & $8.3 E+01$ & $3.6 \mathrm{E}+01$ & 2. $2 \mathrm{E}+01$ & 1. $4 \mathrm{E}+01$ & $6.2 \mathrm{E}+0 \odot$ & 1. $9 \mathrm{E}+0 \odot$ \\
\hline NW & $\odot . \odot \mathrm{E}+\odot \odot$ & $7.1 \mathrm{E}+01$ & $\odot . \odot E+\odot \odot$ & $1.8 \mathrm{E}+01$ & 1. $2 \mathrm{E}+01$ & $5.2 \mathrm{E}+00$ & 1. $5 \mathrm{E}+0 \odot$ \\
\hline WNW & $\odot . \odot \mathrm{E}+\odot \odot$ & $\odot . \odot E+\odot \odot$ & $\odot . \odot E+\odot \odot$ & $\odot . \odot E+\odot \odot$ & 1. $1 \mathrm{E}+01$ & 4. . $7 \mathrm{E}+0 \odot$ & 1. $4 \mathrm{E}+0 \odot$ \\
\hline W & $2.8 \mathrm{E}+02$ & $\odot . \odot \mathrm{OE}+\odot \odot$ & 2. $3 \mathrm{E}+01$ & $1.3 E+01$ & $8.7 \mathrm{E}+\odot \odot$ & $3.8 \mathrm{E}+00$ & 1. $1 \mathrm{E}+00$ \\
\hline WSW & $2.5 \mathrm{E}+02$ & 4. 7E+01 & 2. $1 \mathrm{E}+01$ & 1. $2 \mathrm{E}+01$ & 8. $\odot \mathrm{E}+\odot \odot$ & $3.6 \mathrm{E}+00$ & 1. $1 \mathrm{E}+00$ \\
\hline SW & $3.0 \mathrm{E}+02$ & $5.6 \mathrm{E}+01$ & $2.5 \mathrm{E}+01$ & $1.5 \mathrm{E}+01$ & $9.6 \mathrm{E}+0 \odot$ & 4. $3 E+0 \odot$ & 1. $4 \mathrm{E}+0 \odot$ \\
\hline SSW & $4.0 \mathrm{E}+02$ & $\odot . \odot E+\odot \odot$ & 3. $3 E+01$ & $2.0 \mathrm{E}+01$ & 1. $3 E+01$ & $5.9 \mathrm{E}+00$ & 1. $9 \mathrm{E}+0 \odot$ \\
\hline S & $3.2 E+02$ & $5.9 E+01$ & $2.6 \mathrm{E}+01$ & $1.6 \mathrm{E}+01$ & $1.0 \mathrm{E}+01$ & 4. $7 \mathrm{E}+0 \odot$ & 1. $5 \mathrm{E}+0 \odot$ \\
\hline SSE & $\odot . \odot \mathrm{E}+\odot \odot$ & $4.5 E+01$ & 2. $\odot E+\Theta 1$ & 1. $2 \mathrm{E}+01$ & $7.9 \mathrm{E}+0 \odot$ & $3.6 \mathrm{E}+00$ & 1. $2 \mathrm{E}+0 \odot$ \\
\hline SE & $2.6 \mathrm{E}+02$ & 4. $7 \mathrm{E}+01$ & $2.1 \mathrm{E}+01$ & 1. $2 \mathrm{E}+01$ & $8.3 E+0 \odot$ & $3.8 \mathrm{E}+0 \odot$ & 1. $2 \mathrm{E}+0 \odot$ \\
\hline ESE & $2.8 E+02$ & $5.1 E+01$ & 2. $3 E+01$ & $1.3 E+01$ & $8.8 E+00$ & 4. $. \mathrm{E}+0 \odot$ & 1. $2 \mathrm{E}+0 \odot$ \\
\hline$E$ & $3.6 E+02$ & $6.7 E+01$ & 3. $. E+01$ & $1.8 \mathrm{E}+01$ & 1. 1E+01 & $5.1 \mathrm{E}+0 \odot$ & 1. $5 \mathrm{E}+0 \odot$ \\
\hline ENE & $5.2 E+02$ & $9.6 \mathrm{E}+01$ & 4. $2 \mathrm{E}+01$ & $2.5 \mathrm{E}+01$ & $1.6 \mathrm{E}+01$ & $7.3 E+0 \odot$ & 2. $2 \mathrm{E}+0 \odot$ \\
\hline NE & $\odot . \odot \mathrm{O}+\odot \odot$ & 1. $2 \mathrm{E}+02$ & 5. $3 E+01$ & $3.1 \mathrm{E}+01$ & 2. $\odot E+\odot 1$ & $9.1 \mathrm{E}+0 \odot$ & 2. $8 \mathrm{E}+0 \odot$ \\
\hline NNE & $\odot . \odot \mathrm{E}+\odot \odot$ & 1. $2 \mathrm{E}+02$ & $5.5 \mathrm{E}+01$ & $3.3 E+01$ & 2. $1 \mathrm{E}+01$ & $9.6 \mathrm{E}+00$ & 2. $9 \mathrm{E}+0 \odot$ \\
\hline
\end{tabular}

Distance $(\mathrm{m})$

$\begin{array}{llll}\text { Direction } & 40250 \quad 56350 & 72200\end{array}$

$\begin{array}{rlll}\mathrm{N} & 1.3 \mathrm{E}+0 \odot & 6.6 \mathrm{E}-01 & 3.3 \mathrm{E}-01 \\ \mathrm{NNW} & 7.6 \mathrm{E}-01 & 3.6 \mathrm{E}-01 & 1.7 \mathrm{E}-01 \\ \mathrm{NW} & 6.1 \mathrm{E}-01 & 2.9 \mathrm{E}-01 & 1.3 \mathrm{E}-01 \\ \mathrm{WNW} & 5.6 \mathrm{E}-01 & 2.7 \mathrm{E}-01 & 1.3 \mathrm{E}-01 \\ \mathrm{~W} & 4.7 \mathrm{E}-01 & 2.2 \mathrm{E}-01 & 1.1 \mathrm{E}-01 \\ \mathrm{WSW} & 4.5 \mathrm{E}-01 & 2.2 \mathrm{E}-01 & 1.1 \mathrm{E}-01 \\ \mathrm{SW} & 5.8 \mathrm{E}-01 & 2.9 \mathrm{E}-01 & 1.5 \mathrm{E}-01 \\ \mathrm{SSW} & 8.2 \mathrm{E}-01 & 4.2 \mathrm{E}-01 & 2.2 \mathrm{E}-01 \\ \mathrm{~S} & 6.4 \mathrm{E}-01 & 3.3 \mathrm{E}-01 & 1.8 \mathrm{E}-01 \\ \mathrm{SSE} & 5.0 \mathrm{E}-01 & 2.6 \mathrm{E}-01 & 1.4 \mathrm{E}-01 \\ \mathrm{SE} & 5.2 \mathrm{E}-01 & 2.7 \mathrm{E}-01 & 1.5 \mathrm{E}-01 \\ \mathrm{ESE} & 5.2 \mathrm{E}-01 & 2.7 \mathrm{E}-01 & 1.4 \mathrm{E}-01 \\ \mathrm{E} & 6.5 \mathrm{E}-01 & 3.2 \mathrm{E}-01 & 1.6 \mathrm{E}-01 \\ \mathrm{ENE} & 9.2 \mathrm{E}-01 & 4.5 \mathrm{E}-01 & 2.2 \mathrm{E}-01 \\ \mathrm{NE} & 1.2 \mathrm{E}+0 \odot & 5.6 \mathrm{E}-01 & 2.8 \mathrm{E}-01 \\ \mathrm{NNE} & 1.2 \mathrm{E}+0 \odot & 5.9 \mathrm{E}-01 & 2.9 \mathrm{E}-01\end{array}$

D - 12 
COLLECTIVE EFFECTIVE DOSE EQUIVALENT (person rem/y) (All Radionuclides and Pathways)

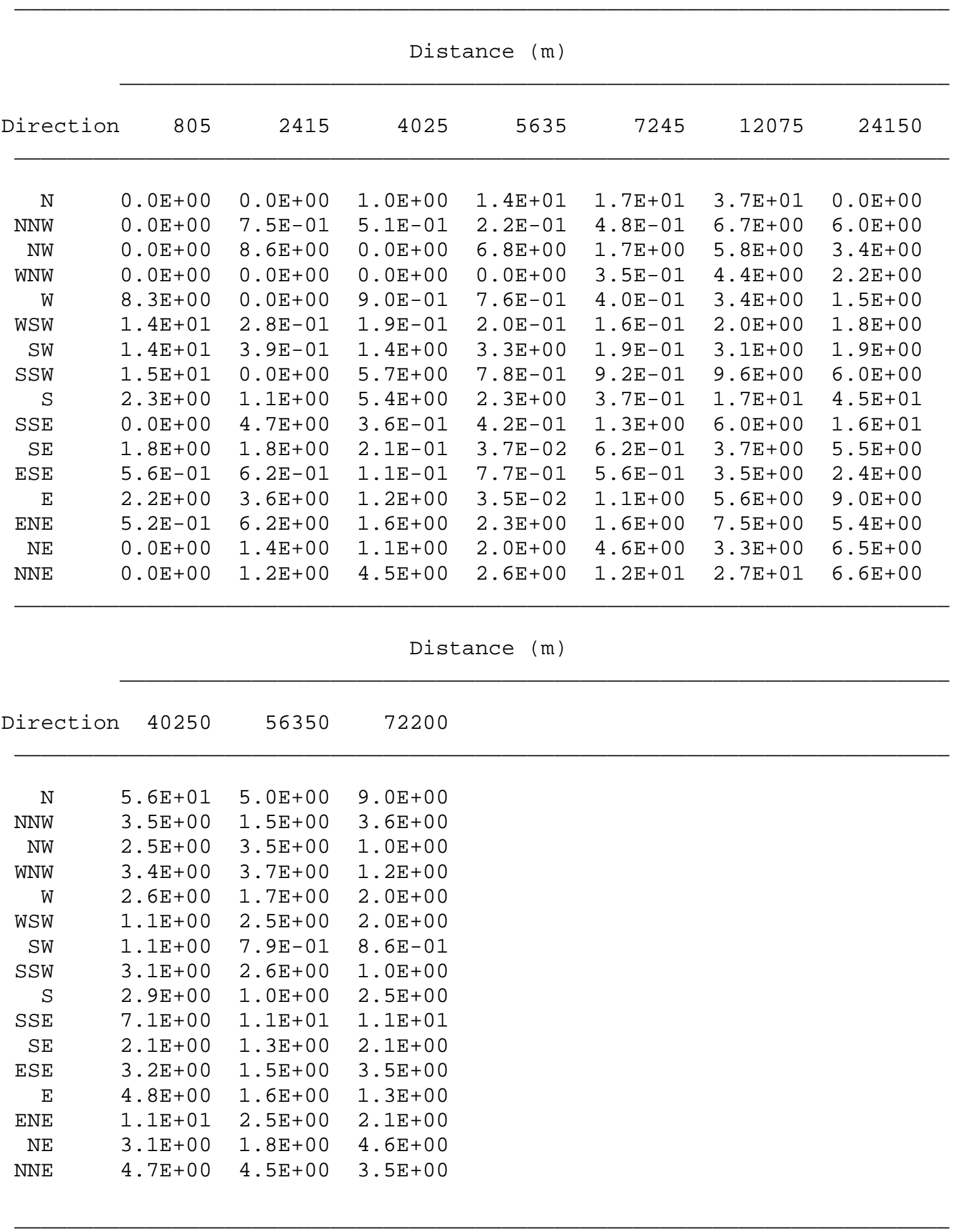


INDIVIDUAL LIFETIME RISK (deaths)

(All Radionuclides and Pathways)

\begin{tabular}{|c|c|c|c|c|c|c|c|}
\hline \multirow[b]{2}{*}{ Direction } & \multicolumn{6}{|c|}{ Distance (m) } & \multirow[b]{2}{*}{24150} \\
\hline & 805 & 2415 & 4025 & 5635 & 7245 & 12075 & \\
\hline $\mathrm{N}$ & $\odot . \odot E+\odot \odot$ & $\odot . \odot E+\odot \odot$ & 4. 3E- 05 & $2.6 E-05$ & 1.7E- 05 & $7.7 E-06$ & $\odot . \odot E+\odot \odot$ \\
\hline NNW & $\odot . \odot \mathrm{E}+\odot \odot$ & $6.6 E-05$ & $2.9 \mathrm{E}-05$ & $1.7 \mathrm{E}-05$ & 1.1E- 05 & 4. $9 \mathrm{E}-06$ & 1. $4 \mathrm{E}-06$ \\
\hline NW & $\odot . \odot \mathrm{E}+\odot \odot$ & $5.6 E-05$ & $\odot . \odot E+\odot \odot$ & 1. $4 \mathrm{E}-05$ & $9.4 E-06$ & 4.1E- 06 & 1. $2 \mathrm{E}-06$ \\
\hline WNW & $\odot .0 \mathrm{E}+0 \odot$ & $\odot . \odot E+\odot \odot$ & $\odot . \odot E+\odot \odot$ & $\odot .0 \mathrm{E}+0 \odot$ & $8.5 E-06$ & $3.7 E-06$ & 1.1E-06 \\
\hline W & $2.2 E-04$ & $\odot . ० E+\odot \odot$ & $1.8 E-05$ & 1.1E- 05 & $6.8 E-06$ & $3.0 E-06$ & $8.7 E-07$ \\
\hline WSW & $2.0 \mathrm{E}-04$ & $3.7 E-05$ & 1. $6 \mathrm{E}-05$ & $9.6 E-06$ & $6.3 E-06$ & $2.8 E-06$ & $8.2 E-07$ \\
\hline SW & $2.4 E-04$ & $4.4 E-05$ & 1. $9 \mathrm{E}-05$ & 1. $2 E-05$ & $7.6 E-06$ & $3.4 \mathrm{E}-06$ & 1. $0 E-06$ \\
\hline SSW & $3.2 E-04$ & $\odot . \odot E+\odot \odot$ & $2.6 \mathrm{E}-\odot 5$ & $1.5 E-05$ & 1. $0 \mathrm{E}-05$ & $4.6 E-06$ & $1.5 E-06$ \\
\hline S & $2.6 E-04$ & $4.7 E-05$ & $2.1 E-05$ & 1. $2 E-05$ & $8.1 E-06$ & $3.7 E-06$ & 1.1E-06 \\
\hline SSE & $\odot .0 \mathrm{E}+0 \odot$ & $3.6 E-05$ & 1. $6 \mathrm{E}-05$ & $9.4 E-06$ & $6.2 E-06$ & $2.8 E-06$ & $8.8 E-07$ \\
\hline SE & $2.1 E-04$ & $3.7 E-05$ & 1. $6 \mathrm{E}-05$ & $9.8 E-06$ & $6.5 E-06$ & $2.9 E-06$ & $9.2 \mathrm{E}-07$ \\
\hline ESE & $2.2 E-04$ & $4.1 E-05$ & 1. $8 \mathrm{E}-\odot 5$ & 1.1E- 05 & $6.9 E-06$ & $3.1 E-06$ & $9.4 \mathrm{E}-07$ \\
\hline$E$ & $2.9 E-04$ & $5.3 E-05$ & $2.3 E-\odot 5$ & 1. $4 \mathrm{E}-05$ & $9.0 E-06$ & 4. $\odot E-\odot 6$ & 1. $2 \mathrm{E}-06$ \\
\hline ENE & $4.1 E-04$ & $7.6 E-05$ & $3.3 E-05$ & $2.0 E-05$ & 1. 3E- 05 & $5.7 E-06$ & 1. $7 \mathrm{E}-06$ \\
\hline NE & $\odot . \odot \mathrm{E}+\odot \odot$ & $9.5 E-05$ & $4.2 \mathrm{E}-05$ & $2.5 E-05$ & $1.6 E-05$ & $7.1 \mathrm{E}-06$ & 2. 1E- 06 \\
\hline NNE & $\odot .0 \mathrm{E}+\odot \odot$ & $9.9 E-05$ & 4. 3E- 05 & $2.6 E-05$ & 1. 7E- 05 & $7.5 E-06$ & 2. 3E- - 6 \\
\hline
\end{tabular}

Distance (m)

$\begin{array}{llll}\text { Direction } & 40250 \quad 56350 & 72200\end{array}$

$\begin{array}{rlll}N & 1.0 \mathrm{E}-06 & 5.0 \mathrm{E}-07 & 2.4 \mathrm{E}-07 \\ \mathrm{NNW} & 5.8 \mathrm{E}-07 & 2.7 \mathrm{E}-07 & 1.2 \mathrm{E}-07 \\ \mathrm{NW} & 4.6 \mathrm{E}-07 & 2.1 \mathrm{E}-07 & 8.9 \mathrm{E}-08 \\ \mathrm{WNW} & 4.3 \mathrm{E}-07 & 2.0 \mathrm{E}-07 & 8.5 \mathrm{E}-08 \\ \mathrm{~W} & 3.5 \mathrm{E}-07 & 1.6 \mathrm{E}-07 & 7.3 \mathrm{E}-08 \\ \mathrm{WSW} & 3.4 \mathrm{E}-07 & 1.6 \mathrm{E}-07 & 7.7 \mathrm{E}-08 \\ \mathrm{SW} & 4.4 \mathrm{E}-07 & 2.1 \mathrm{E}-07 & 1.0 \mathrm{E}-07 \\ \mathrm{SSW} & 6.3 \mathrm{E}-07 & 3.1 \mathrm{E}-07 & 1.6 \mathrm{E}-07 \\ \mathrm{~S} & 4.8 \mathrm{E}-07 & 2.4 \mathrm{E}-07 & 1.2 \mathrm{E}-07 \\ \mathrm{SSE} & 3.8 \mathrm{E}-07 & 1.9 \mathrm{E}-07 & 9.8 \mathrm{E}-08 \\ \mathrm{SE} & 3.9 \mathrm{E}-07 & 2.0 \mathrm{E}-07 & 1.0 \mathrm{E}-07 \\ \mathrm{ESE} & 3.9 \mathrm{E}-07 & 1.9 \mathrm{E}-07 & 9.5 \mathrm{E}-08 \\ \mathrm{E} & 4.9 \mathrm{E}-07 & 2.3 \mathrm{E}-07 & 1.1 \mathrm{E}-07 \\ \mathrm{ENE} & 7.0 \mathrm{E}-07 & 3.3 \mathrm{E}-07 & 1.6 \mathrm{E}-07 \\ \mathrm{NE} & 8.8 \mathrm{E}-07 & 4.2 \mathrm{E}-07 & 2.0 \mathrm{E}-07 \\ \mathrm{NNE} & 9.3 \mathrm{E}-07 & 4.4 \mathrm{E}-07 & 2.1 \mathrm{E}-07\end{array}$


COLLECTIVE FATAL CANCER RATE (deaths/y)

(All Radionuclides and Pathways)

\begin{tabular}{|c|c|c|c|c|c|c|c|}
\hline \multirow[b]{2}{*}{ Direction } & \multicolumn{7}{|c|}{ Distance $(\mathrm{m})$} \\
\hline & 805 & 2415 & 4025 & 5635 & 7245 & 12075 & 24150 \\
\hline $\mathrm{N}$ & $\odot . \odot E+\odot \odot$ & $\odot . \odot E+\odot \odot$ & $1.1 E-05$ & $1.5 E-\odot 4$ & $1.8 E-04$ & $3.8 E-04$ & $\odot . \odot E+\odot \odot$ \\
\hline NNW & $\odot . \odot \mathrm{Q}+\odot \odot$ & $7.7 E-06$ & $5.2 E-06$ & $2.2 \mathrm{E}-06$ & $4.9 E-06$ & $6.8 E-05$ & $6.0 \mathrm{E}-05$ \\
\hline NW & $\odot . \odot \mathrm{E}+\odot \odot$ & $8.8 E-05$ & $\odot . \odot E+\odot \odot$ & $6.9 E-05$ & 1. $7 E-05$ & $5.9 E-05$ & $3.5 \mathrm{E}-05$ \\
\hline WNW & $\odot . \odot \mathrm{O}+\odot \odot$ & $\odot . \odot E+\odot \odot$ & $\odot . \odot E+\odot \odot$ & $\odot . \odot E+\odot \odot$ & $3.6 E-06$ & $4.4 E-05$ & $2.2 \mathrm{E}-05$ \\
\hline W & $8.6 \mathrm{E}-05$ & $\odot . \odot E+\odot \odot$ & $9.2 E-06$ & $7.8 E-06$ & $4.1 E-06$ & $3.4 E-05$ & $1.5 \mathrm{E}-05$ \\
\hline WSW & $1.5 E-04$ & $2.9 E-06$ & 1. $9 \mathrm{E}-06$ & $2.0 \mathrm{E}-06$ & $1.6 \mathrm{E}-06$ & $2.0 E-05$ & $1.8 \mathrm{E}-05$ \\
\hline SW & $1.4 E-04$ & 4. $0 \mathrm{E}-06$ & $1.4 E-05$ & $3.3 E-05$ & 2. $0 \mathrm{E}-\odot 6$ & $3.1 E-05$ & 1. $9 \mathrm{E}-05$ \\
\hline SSW & $1.6 E-04$ & $\odot . \odot E+\odot \odot$ & $5.8 E-05$ & $8.0 \mathrm{E}-06$ & $9.4 E-06$ & $9.8 E-05$ & $6.1 \mathrm{E}-05$ \\
\hline S & $2.3 E-05$ & 1.1E- 05 & $5.5 E-05$ & $2.3 E-05$ & $3.8 E-06$ & $1.7 E-04$ & $4.5 E-04$ \\
\hline SSE & $\odot . \odot \mathrm{E}+\odot \odot$ & $4.8 E-05$ & $3.7 E-06$ & $4.3 E-06$ & 1. $4 \mathrm{E}-05$ & $6.0 \mathrm{E}-05$ & $1.6 \mathrm{E}-04$ \\
\hline SE & $1.9 E-05$ & 1.9E- 05 & $2.1 \mathrm{E}-06$ & $3.8 E-07$ & $6.3 E-06$ & $3.7 E-05$ & $5.5 E-05$ \\
\hline ESE & $5.7 E-06$ & $6.3 E-06$ & $1.2 E-06$ & $7.8 E-06$ & $5.7 E-06$ & $3.5 E-05$ & $2.4 \mathrm{E}-05$ \\
\hline E & $2.3 E-05$ & $3.7 E-\odot 5$ & $1.2 E-05$ & $3.6 \mathrm{E}-07$ & 1.1E- 05 & $5.7 E-05$ & $9.0 \mathrm{E}-05$ \\
\hline ENE & $5.3 E-06$ & $6.4 \mathrm{E}-05$ & $1.6 \mathrm{E}-05$ & $2.4 \mathrm{E}-05$ & $1.6 \mathrm{E}-05$ & $7.6 \mathrm{E}-05$ & $5.4 \mathrm{E}-05$ \\
\hline NE & $\odot . \odot E+\odot \odot$ & $1.5 E-05$ & $1.1 \mathrm{E}-05$ & $2.0 \mathrm{E}-05$ & $4.7 E-\odot 5$ & $3.3 E-05$ & $6.5 \mathrm{E}-05$ \\
\hline NNE & $\odot . \odot E+\odot \odot$ & 1. 3E- 05 & $4.6 E-05$ & $2.6 \mathrm{E}-05$ & 1. $2 E-\odot 4$ & $2.7 E-\odot 4$ & $6.7 E-05$ \\
\hline \multicolumn{8}{|c|}{ Distance (m) } \\
\hline Direction & 40250 & 56350 & 72200 & & & & \\
\hline $\mathrm{N}$ & $5.6 E-04$ & $4.8 E-05$ & $8.6 E-05$ & & & & \\
\hline NNW & $3.5 E-05$ & 1. $4 \mathrm{E}-05$ & $3.2 E-05$ & & & & \\
\hline NW & $2.5 E-05$ & 3. 3E- 05 & $8.8 E-06$ & & & & \\
\hline WNW & $3.3 E-05$ & $3.5 E-05$ & 1.1E-05 & & & & \\
\hline W & $2.5 E-05$ & $1.6 \mathrm{E}-05$ & $1.7 E-\odot 5$ & & & & \\
\hline WSW & 1.1E- -05 & $2.3 E-05$ & $1.7 E-05$ & & & & \\
\hline SW & $1.1 \mathrm{E}-05$ & $7.5 \mathrm{E}-06$ & $7.7 \mathrm{E}-06$ & & & & \\
\hline SSW & $3.0 E-05$ & $2.5 E-05$ & $9.5 E-06$ & & & & \\
\hline S & $2.8 E-05$ & $9.5 \mathrm{E}-06$ & $2.2 E-05$ & & & & \\
\hline SSE & $6.9 E-05$ & $1.1 E-04$ & $9.7 \mathrm{E}-05$ & & & & \\
\hline SE & $2.1 E-05$ & $1.2 E-05$ & 1.9E-05 & & & & \\
\hline ESE & $3.1 \mathrm{E}-05$ & 1. $4 \mathrm{E}-05$ & $3.1 E-05$ & & & & \\
\hline E & $4.7 E-05$ & $1.5 \mathrm{E}-05$ & 1. $2 \mathrm{E}-05$ & & & & \\
\hline ENE & $1.1 \mathrm{E}-04$ & $2.4 E-05$ & 1.9E-05 & & & & \\
\hline NE & $3.0 E-05$ & $1.8 E-05$ & $4.3 E-05$ & & & & \\
\hline NNE & $4.7 E-05$ & $4.4 E-05$ & $3.3 E-05$ & & & & \\
\hline
\end{tabular}


